\author{
UNIVERSIDADE DE SÃO PAULO \\ Instituto de Física
}

\title{
Estudo de processos a energias médias e altas pelo método Monte Carlo
}

\author{
Israel Ariel González Medina
}

Orientador: Dr Airton Deppman

Tese de doutorado apresentada ao Instituto de Física para a obtenção do título de Doutor em Ciências

Comissão Examinadora:

Prof. Dr. Airton Deppman (IFUSP)

Prof. Dr. Marcelo Munhoz (IFUSP)

Prof. Dr. Gastao Krein (UNESP)

Prof. Dr. Sérgio José Barbosa Duarte (CBPF)

Prof. Dr. Bruno El-Bennich (Universidade Cruzeiro do Sul)

São Paulo 
"Las verdades elementales caben en el ala de un colibrí."

José Martí. 


\title{
UNIVERSIDADE DE SÃO PAULO
}

\author{
Resumo \\ Instituto de Física \\ Departamento de Física Experimental \\ Doutorando em Ciências
}

O trabalho de pesquisa está orientado ao estudo de processos nucleares em altas energias usando o modelo de cascata intranuclear desenvolvido no código CRISP. O estudo é realizado pela introdução de modificações no código CRISP, visando adaptá-lo à utilização em cálculos relacionados às reações nucleares onde a densidade de hádrons é quente e densa. Este estado é muito sensível às modificações no meio da distribuição dos mésons vetoriais $\rho$ e $\omega, \phi$ e $J / \Psi$. Em altas energias, o código CRISP incorpora os processos de fotoprodução de mésons vetoriais e os processos de interação no estado final destes mésons com o núcleo. A cascata intranuclear que se formará será processada de acordo com o modelo já utilizado no código, com as devidas correções que se fizerem necessárias. A nova extensão de CRISP é utilizada para estudar as fotorreações nucleares em altas energias e na análise de efeitos importantes como a multiplicidade de partículas secundários produzidas, o efeito de sombreamento, a fotoprodução sub-limiar de mésons, a distribução de massa dos méson gerados na matéria nuclear e a transparência. Usando o CRISP, as características do núcleo residual formado ao final da reação poderão ser determinadas e comparadas com os dados experimentais. A boa concordância entre estes e nossos resultados mostram o potencial do modelo CRISP nesta nova versão. 


\title{
UNIVERSIDADE DE SÃO PAULO
}

\author{
Abstract \\ Instituto de Física \\ Departamento de Física Experimental \\ Doutorando em Ciências
}

We present recent improvements in the CRISP code for nuclear reactions simulation for study high energy nuclear reactions. In nuclear environment, at these energies, hadron density is hot, dense and very sensitive to the modification of vector meson distribution. The photoproduction of vector mesons $\rho$ e $\omega, \phi$ and $J / \Psi$ was included in the code, also the interaction of these mesons with the nucleus. Fotonuclear reactions are treated according the multicollisional model included in the CRISP code. Nuclear effects as shadowing, subthreshold production, Pauli Blocking, meson invariant mass distribution and transparency can be studied with this CRISP. The model describe in details all the features of the nucleus at the end of the nuclear reaction allowing compare some important quantities with experimental data. The agreement of the results with the data set is a prove of the potentialities of our code. 


\section{Agradecimentos}

Esse tese não seria realizada sem o apoio e a ajuda de meus pais e irmã. Eu devo tudo para eles. Agradeçõ ao meu orientador, prof. Dr. Airton Deppman e ao prof. Dr. Fernando Guzman, que instruíram meus passos no percorrer no caminho da ciência, sempre com dedicação. A todos os professores que a partir da minha infância e ao longo da minha vida, contribuíram na minha formação. Ao professor Luis Ramón do IPVEC Carlos Roloff que dedicou noites após noites para me treinar para as olimpíadas de física. O interesse pelo mundo das equações e as teorias nasceu ali. Eles faziam mais do que seu trabalho porque mais que ensinar, eles educavam e acreditavam. Não esqueço nenhum deles. Sua contribuição é inestimável. Agradeçõ a minha família toda que fez deste trabalho sua preocupação, suas lágrimas e sucessos. A meus amigos de sempre e de agora Yoelvis, Aley, Yaicel, Julio, Johan, Yasser, Juano, Camilo, Yadira que sempre estarão onde eu precisar. Aos colegas Mc. Evandro, Lucas Marquez, Júlio César e Dr. Pedro Rossi pela sua amizade e ajuda incondicional na programação, idioma. Agradeço à Universidades de São Paulo e o Instituto de Física por permitir usar suas instalações para fazer o doutorado, à agencia CNPQ pelo suporte (Grants No. 1141168/2010-2) e ao Brasil todo por me acolher como mais um.

\section{Obrigado!}




\section{Sumário}

Resumo ii

Abstract iii

Agradecimentos iv

Índice de Figuras viii

Índice de Tabelas $\quad$ xii

$\begin{array}{ll}\text { Abreviaturas } & \text { xiii }\end{array}$

1 Introdução 1

2 O Modelo de Cascata Intranuclear e Evaporação/Fissão 11

2.1 Modelo da cascata intranuclear CRISP . . . . . . . . . . . . . . . . . 11

$2.1 .1 \quad \mathrm{O}$ estado fundamental . . . . . . . . . . . . . . . . . 12

2.1.1.1 Distribução da posição e dos momentos dos nucleons . . . 12

2.1.1.2 Potencial nuclear e barreira de Coulomb . . . . . . . . . 15

2.1.1.3 O efeito do potencial nuclear. Massa efetiva dos nucleons ligados ......................... 16

2.1.1.4 O mecanismo de bloqueio de Pauli . . . . . . . . . . . 17

2.1.1.5 Ressonâcias nucleônicas . . . . . . . . . . . . . . 20

2.1.2 A dinâmica da cascata intranuclear . . . . . . . . . . . . . . . . . . 21

2.1.2.1 A evolução temporal da cascata . . . . . . . . . . . . 21

2.1.2.2 Emissões de pré-equilíbrio e balanço energético . . . . . . 22

2.1.2.3 O critério energético de parada e o cálculo da energia de excitação . . . . . . . . . . . . . . . . . . . . . . . . . . . 24

2.1.3 Efeito de sombreamento . . . . . . . . . . . . . . . . . 26

2.2 Modelo de Evaporação/ Fissão . . . . . . . . . . . . . . . . . . . . . . . 27

2.2.1 Descrição do Modelo de Evaporação/ Fissão . . . . . . . . . . . . . 28

3 Colisões de partículas a altas energias $\quad 32$

3.1 Introdução . . . . . . . . . . . . . . . . . . . 32

3.2 Espalhamento de uma partícula pelo um potencial central . . . . . . . . 36 
3.2 .1 A aproximação da equação de Schrödinger . . . . . . . . . . . . . . 36

3.2 .2 A aproximação da matriz $\mathrm{S} \ldots \ldots . \ldots . \ldots . . \ldots 38$

3.3 A matriz $\mathrm{S}$ relativística . . . . . . . . . . . . . . . . . 41

3.3 .1 Unitariedade . . . . . . . . . . . . . . . . . . . 44

3.3 .2 Analiticidade . . . . . . . . . . . . . . . . . 45

3.3 .3 Crossing . . . . . . . . . . . . . . . . . . . . . . . . . . . . . . . . 58

3.4 Teoria de Regge . . . . . . . . . . . . . . . . . . . . . 53

3.5 Abordagem matemática da teoria de Regge. . . . . . . . . . . . . . 55

3.6 Seção de choque elástica e total. . . . . . . . . . . . . . . . . 60

3.7 O Pomeron e o Odderon. . . . . . . . . . . . . . . . . . . . 62

3.8 Introdução à física das colisões ultraperiféricas . . . . . . . . . . . . . . . . . 65

3.9 A física das colisões ultraperiférica no LHC . . . . . . . . . . . . . . . . 67

3.10 Efeitos electromagnéticos em colisões de íons relativísticas . . . . . . . . 70

3.11 Produção de ressonâncias gigantes nas colisões ultraperiféricas . . . . . . . 72

3.12 Produção de píons nas colisões ultraperiféricas . . . . . . . . . . . . . . . . 73

3.13 Mecanismo de interação inicial . . . . . . . . . . . . . . . . . . . . . 75

3.13 .1 Seção de choque . . . . . . . . . . . . . . . 77

4 Fótoprodução dos mésons vetoriais no ambiente nuclear. $\quad \mathbf{7 9}$

4.1 Fotoprodução de mésons vetoriais pelo modelo de soft dipole Pomeron . . 79

4.1 .1 Seção de choque total . . . . . . . . . . . . . . . . 83

4.1 .2 Seção de choque diferencial . . . . . . . . . . . . . . . . . . . . . 84

4.2 Interações no estado final (FSI) dos mésons . . . . . . . . . . . . . 85

4.2.1 O modelo de troca de um méson no estado final da reação $\omega N$. . 86

4.2.2 O modelo de troca de um méson para a dissociação do méson $J / \Psi \quad 92$

4.2.3 O modelo ressonante no estado final da reação $\pi N$. . . . . . . 95

4.2.4 Interação no estado final dos mésons vectoriais

$\pi, \rho, \omega, \phi$ e $J / \Psi$ incluída no código CRISP . . . . . . . . . 96

5 Resultados

5.1 Fotoprodução de mésons vetoriais. Seção de choque de fotoprodução vs número de massa . . . . . . . . . . . . . . . . . . . . . . . . 100

5.2 Fotoprodução de mésons vetoriais. Seção de choque de fotoprodução do nucleon ligado vs energia . . . . . . . . . . . . . . . . . 102

5.3 Fotoprodução de mésons vetoriais abaixo do limiar de produção . . . . . . 103

5.3 .1 Movimento fermiônico . . . . . . . . . . . . . . . . . . 104

5.3.2 Mecanismo de bloqueio de Pauli . . . . . . . . . . . . . . 107

5.3 .3 Comportamento sub-limiar de $A_{\text {eff }}(E) \ldots \ldots$. . . . . . . . 107

5.3 .4 Fotoprodução do méson $\mathrm{J} / \Psi$ perto ao limiar . . . . . . . . . . . . . . . . . . . . . . . . .

5.4 Largura do nucleo composto $V-A \ldots \ldots \ldots$

5.5 O espectro de massa do méson $\rho \ldots \ldots$. . . . . . . . . . . . 113

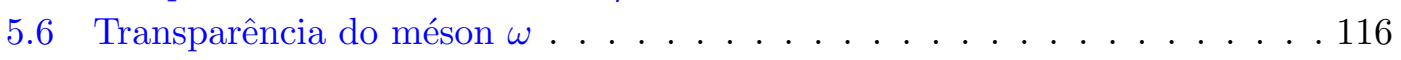

6 Conclusões e próximos passos 
Referências Bibliográficas 


\section{Lista de Figuras}

1.1 Representação do mecanismo de cascata intranuclear. Figura extraída da

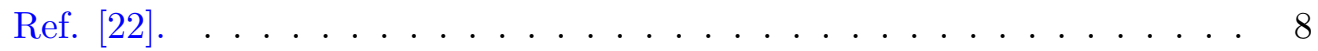

2.1 Estrutura em camadas no espaço dos momentos para simular o mecanismo de Pauli $[22] . \ldots \ldots \ldots \ldots \ldots$

2.2 Diagrama do potencial nuclear no modelo de gás de Fermi . . . . . . . . . 16

2.3 Distribuição do número de ocupação para prótons para vários instantes da cascata comparado com o ajuste da função de partição na temperatura. Figura obtida da Ref. [92] . . . . . . . . . . . . . . . . . . . . 18

2.4 Distribuição do número de ocupação para prótons para vários instantes da cascata. Figura obtida da Ref. [92] . . . . . . . . . . . . . . . 19

2.5 Distribuição do número de ocupação para nêutrons para vários instantes da cascata comparado com o ajuste da função de partição na temperatura. Figura obtida da Ref. [92]. . . . . . . . . . . . . . . . . . . . . . . 19

2.6 Distribuição do número de ocupação para nêutrons para vários instantes da cascata. Figura obtida da Ref. [92] . . . . . . . . . . . . . . . 20

2.7 Representação de um processo fictício em que um fóton transfere toda sua energia a um nucleon e escapa do núcleo sem interagir com os outros nucleons . . . . . . . . . . . . . . . . . . 2 25

2.8 Diagrama do efeito de sombreamento não saturado do fóton no núcleo. . . 27

2.9 Diagrama do efeito de sombreamento saturado do fóton no núcleo. . . . . 27

2.10 Sessão de choque da fotofissão na região de ressonância nucleônica obtida com o código CRISP comparada com dados experimentais. Figura publicada na Ref. [17]. . . . . . . . . . . . . . . . . . . . . . . 28

2.11 Diagrama do processo de competição evaporação/fissão que acontece no núcleo residual simulado no CRISP. Figura publicada na Ref. [92]. . . . . 29

3.1 Difração hadrônica. Elástico (esquerda), difração única (centro), difração dupla (direita) . . . . . . . . . . . . . . . . . . 34

3.2 Espalhamento de uma partícula por um potencial central [106] . . . . . . 37

3.3 Diagrama da condição de unitariedade da amplitude de espalhamento [106]. 44

3.4 Representação gráfica do teorema óptico para o espalhamento relativística [106] . . . . . . . . . . . . . . . . . . . . 4 45

3.5 Representação gráfica do espalhamento sucessivo por um potencial. . . . . 47

3.6 Singularidades da matriz de espalhamento frontal de duas partículas [106]. 48

3.7 Contorno da integral de Cauchy da amplitude $A(s, t)[106] \ldots$. . . . . . 49

3.8 Seções de choque totais das colisões $p p$ e $p \bar{p}$ ajustadas por A. Donnachie e P. V. Landshoff usando a teoría de Regge que demostram o teorema de Pomeranchuk [114] . . . . . . . . . . . . . . . . . . . 52 
3.9 Gráfico das trajetórias da partículas $\mathrm{N}$ e $\Delta[106] \ldots \ldots \ldots$. . . . . . 53

3.10 Diagramas da troca do Reggeon $\rho$ na colisão $\pi^{-} p \rightarrow \pi^{0} n$. . . . . . . . 54

3.11 Interpolação da trajetória mesônica para las partículas $f_{2}, a_{2}, \rho, \omega[106]$. 59

3.12 Seções de choque totais das colisões $a) p p$ e $p \bar{p}, b) \pi^{-} p$ e $\left.\pi^{+} p, c\right) K^{-} p$ e $K^{+} p$ e d) $\gamma p$ ajustadas usando a teoria de Regge por A. Donnachie e P. V.

Landshoff. Figura publicada na Ref.[114]. . . . . . . . . . . . . . . . 61

3.13 Interpolação da trajetória do pomeron com uma partícula glueball [106]. . 63

3.14 Diagrama esquemático de uma colisão ultraperiféricas entre dois íons. . 65

3.15 Diagrama esquemático: (a) da interação electromagnética onde os fótons dos íons interagem entre eles, (b) da reação fóton-núcleo onde o fóton emitido por um núcleo interage com o outro núcleo, (c) da reação fótonresíduo nuclear onde o fóton emitido por um núcleon interage com o outro núcleo fissionado resultado de uma fotorreação com um núcleo diferente ao inicial. Figura publicada na Ref. [7] . . . . . . . . . . . . . . 66

3.16 Diagrama esquemático do intervalo cinemático das colisão ultraperiféricas no LHC. Figura publicada na Ref. [7]. . . . . . . . . . . . . . . 70

3.17 O fluxo de fótons para uma energia de centro do massa de $\sqrt{s_{N N}}=200$, $G e V$ na colisão $\mathrm{Au}+\mathrm{Au}$ no RHIC e a colisão $\mathrm{Pb}+\mathrm{Pb}$ com energia do centro de massa $\sqrt{s_{N N}}=5, \mathrm{TeV}$ no LHC comparado com a colisão $e A u$ à $10 \mathrm{GeV}+100 \mathrm{GeV}$. O gráfico do RHIV é mostrado multiplicado por 6000. Figura publicada na Ref. [7]. . . . . . . . . . . . . . . .

$3.18 \mathrm{O}$ espectro de fótons para colisões ultraperiféricas dos núcleos $\mathrm{Pb}+\mathrm{Pb}$ e $\mathrm{Ca}+\mathrm{Ca}$ no LHC para o valor da energia de repouso do núcleo alvo. Figura publicada na Ref. [7]. . . . . . . . . . . . . . . . . . 71

3.19 Diagrama das contribuições à seção de choque de produção de ressonâncias na colisão ultraperiférica ${ }^{40} \mathrm{Ca}+{ }^{238} \mathrm{U}$. Na figura, a linha traço-pontilhada corresponde à contribuição magnética, a linha tracejada à dipolar eléctrica, a linha pontilhada à contribuição quadripolar eléctrica e a linha cheia à soma das duas. Figura publicada na Ref. [133]. . . . . . . . . . . . . . 73

3.20 Diagrama das contribuições à seção de choque de produção de ressonâncias na colisão ultraperiférica ${ }^{40} \mathrm{Ca}+{ }^{238} \mathrm{U}$. A figura é uma ampliação da figura 3.19 que só contem a parte eléctrica da contribuição onde a linha tracejada corresponde à contribuição dipolar eléctrica, a linha traço-pontilhada à contribuição quadripolar eléctrica e a linha cheia à soma das duas. Figura publicada na Ref. [133]. . . . . . . . . . . . . . . . . . . . . . . 74

3.21 Diagrama da seção de choque de produção de píons para colisões ultraperiféricas dos núcleos $\mathrm{Pb}+\mathrm{Pb}$ e $\mathrm{Ca}+\mathrm{Ca}$ em função do fator de Lorentz

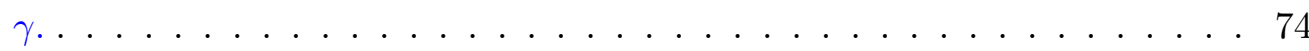

3.22 Distribução de número de fotões em função da energia numa colição ultraperiférica de tipo $A A$ para diferentes valores de parâmetro de impacto mínimo obtida com o código CRISP. . . . . . . . . . . . . . 77

3.23 Luminosidade efetiva $\gamma \gamma$ no LHC (direita) e no RHIC para diferentes colisões de íons. Figura publicada na Ref. [7] . . . . . . . . . . . 78

4.1 Diagrama esquemático da fotoprodução de um méson vectorial. . . . . . . 81

4.2 Seções de choque de fotoprodução por nucleon obtido gerada com o código CRISP em comparação com dados experimentais obtidos de várias fontes. 84

4.3 Seção de choque diferencial de fotoprodução do méson $\rho_{0}, \omega$ e $\phi$ no póton obtida com o código CRISP comparada com dados experimetais [159-161]. 85 
4.4 Seção de choque diferencial de fotoprodução do méson $J / \Psi$ no póton obtida com o código CRISP comparada com dados experimetais [162]. . . 86

4.5 Diagramas dos canais da interação no estado final dos sistema $\omega N$ incluídos no código CRISP. . . . . . . . . . . . . . . . . . . 87

4.6 Seção de choque elástica $\omega N \rightarrow \omega N$. O modelo da troca de um méson com $g_{\omega \sigma \omega}=1.76$ é representado pela línea cheia, o limite dado pelo modelo de quark pela línea pontilhada e a interpolação dos dois modelos pela línea tracejada. Figura extráida da Ref. [165] . . . . . . . . . . . . . . 88

4.7 Seções de choque diferenciais angular para $\pi^{+} n \rightarrow \omega p$. Figura extráida da Ref. [165]. . . . . . . . . . . . . . . . . . . . . 90 90

4.8 Seções de choque diferenciais angular para $\pi^{+} n \rightarrow \omega p$. Figura extráida da Ref. [165]. . . . . . . . . . . . . . . . . . . . . . 91

4.9 Seções de choque diferenciais para $\pi^{+} n \rightarrow \omega p$ e $\pi^{-} p \rightarrow \omega n$. Figura extráida da Ref. [165]. . . . . . . . . . . . . . . . . . . . . 91

4.10 Seções de choque totais para os canais $\pi N \rightarrow \omega N$ e $\omega N \rightarrow \pi N$. Figura extráida da Ref. [165] . . . . . . . . . . . . . . . . . . . . . 92

4.11 Processo de dissociação das interações $J / \Psi N \rightarrow X_{1} X_{2}$ incluídas no código CRISP.

4.12 Processos de produção de mésons vectoriais pelo modelo ressonante incluídos no código CRISP.

4.13 Seções de choque totais da interação $\rho N \rightarrow V N$ (esquerda) e $\omega N \rightarrow V N$ (direita) incluídas no CRISP. . . . . . . . . . . . . . . . 96

4.14 Seções de choque totais da interação $\phi N \rightarrow V N$ (esquerda) e $J / \Psi N \rightarrow$ $V N$ (direita) incluídas no CRISP. . . . . . . . . . . . . . . . . 97

4.15 Seções de choque totais da interação $\pi N \rightarrow V N$ incluídas no CRISP (esquerda) e comparação da seções de choque totais para a reação $\pi N \rightarrow$ $\omega N$ pelos modelos troca de um méson e ressonante (direita). . . . . . . 98

5.1 Seção de choque de fotoprodução de mésons vetoriais em função do número de massa atômica para uma energia do fóton $E_{\gamma=9 \mathrm{GeV}}$. A figura mostra o ajuste geral para todos os mésons pela Equação 5.1 comparada com dados experimentais da Ref. [184]. O melhor ajuste dos parâmetros é $\sigma_{0}=0.98 \pm 0.03$ e $\alpha=0.87 \pm 0.01 \mathrm{com} \chi_{\text {red }}^{2}=3.5 \ldots \ldots$. . . . . . 101

5.2 Seção de choque de fotoprodução do nucleon ligado vs energia obtido com o código CRISP comparada com dados experimentais. . . . . . . . . . . 103

5.3 Flutuação energética dada pelo movimento fermiônico sobre o sistema $\gamma N$ para nêutrons e prótons do núcleo ${ }^{238} \mathrm{U}$ e energia do fóton $\sim 8470 \mathrm{MeV}$ em função do momento no eixo $x, p_{x}$, para diferentes camadas. . . . . . 106

5.4 Efeitos do bloqueio de Pauli na fotoprodução do méson $J / \Psi$ perto ao limiar. Os parâmetros obtidos do melhor ajuste dos dados à equação 5.8 são $\alpha=2.3 \cdot 10^{6} \pm 3.5 \cdot 10^{5}$ e $\beta=-1.7 \pm 0.02 \mathrm{com} \chi_{\text {red }}^{2}=5.5 \ldots . . .108$

5.5 Seção de choque de fotoprodução de méson vetoriais em função do número de massa para varias energias do fóton perto ao limiar. . . . . . . . . . 110

5.6 Comportamento do expoente $\alpha$ na fotoprodução do méson $J / \Psi$ perto ao limiar ajustado à equação $5.9 \ldots$. . . . . . . . . . . . . . . . . 111

5.7 Seção de choque por nucleon ligado do méson $J / \Psi$ perto ao limiar usando duas normalizações: com um efeito de sombreamento saturado e (quadros vermelhos) e com um efeito de sombreamento não saturado dependente da energia (círculos pretos). . . . . . . . . . . . . . . . . . . 11 
5.8 Tempo de dissociação do méson $J / \Psi$ com (quadrados pretos) e sem FSI (círculos vermelhos) na escala da direita. O tempo médio de dissociação com FSI é dado por dois processos mostrados na figura: a emissão do méson fora do núcleo (círculos abertos) e a interação com o nucleons (quadrados abertos). . . . . . . . . . . . . . . . . . 114

5.9 Distribuição da massa invariante do méson $\rho$ gerado no processo de fotoprodução no ${ }^{56} \mathrm{Fe}$ com o código CRISP comparada com dados experimentais da Ref. [191]. . . . . . . . . . . . . . . . . . 115

5.10 Transparência do méson $\omega$ obtida com o código CRISP para $E_{\gamma}=1.7 \mathrm{GeV} .117$ 


\section{Lista de Tabelas}

4.1 Parâmetros obtidos pelo ajuste dos dados experimentais para a fotoprodução dos mésons $\rho, \omega, \phi$ e $J / \Psi$ realizado na Ref. [152] . . . . . . . 83

4.2 Massa efetiva e largura da ressonância bariônica mostradas em (4.52), e os termos $B=B_{\text {in }} B_{\text {out }}(2 J+1)$ obtido pelo ajuste de datos experimentais à equação de Breit-Wigner na Ref. [182]. . . . . . . . . . . . . . . 96

5.1 Valores da seção de choque por nucleon e o expoente $\alpha$ obtido pelo ajuste da seção de choque fotoprodução para vários núcleos com a Equação 5.1. 101 


\section{Abreviaturas}

$\begin{array}{ll}\text { CRISP } & \text { Colaboração Rio de Janeiro - São Paulo } \\ \text { VMD } & \text { Vector Meson Dominance } \\ \text { FSI } & \text { Final State Interaction } \\ \text { MCMC } & \text { Multi-Collisional Monte Carlo } \\ \text { MCEF } & \text { Monte Carlo Evaporation Fission } \\ \text { LHC } & \text { Large Hadron Collider } \\ \text { jLab } & \text { Thomas Jefferson Accelerator Facility } \\ \text { HERA } & \text { Hadron Electron Ring Accelerator } \\ \text { UPCs } & \text { Ultraperipheral Collisions } \\ \text { BDR } & \text { Black Disk Regime } \\ \text { DIS } & \text { Deep Inlelastic Scattering }\end{array}$


Dedicada a mis padres y hermana 


\section{Capítulo 1}

\section{Introdução}

As reações nucleares são ferramentas importantes para o estudo da estrutura nuclear e nucleônica. pois permitem o estudo da formação e propagação de diferentes partículas no ambiente nuclear como ressonâncias bariônicas e hyperons [1-6], o estudo do efeito de shadowing na seção de choque de fotoabsorção dado pela hadronização dos fótons no meio nuclear entre outros processos. Entre as reações nucleares se encontram as reações fotonucleares. O estudo destas tem grande interesse da comunidade científica na atualidade e são estudados nos mais importantes colisores de partículas do mundo como o Large Hadron Collider (LHC) e o Thomas Jefferson Accelerator Facility (JLab)[714]. Uma ferramenta que consiga simular corretamente os processos estudados nestas instalações seria muito importante como suporte teórico na análise dos resultados experimentais, bom como a preparação dos experimentos devido à grande complexidade e o alto custo deles.

As reações fotonucleares que estão caraterizadas por ter uma grande simplicidade já que não produzem grandes modificações na estrutura nuclear comparadas com as produzidas pelas reações induzidas por partículas que interagem fortemente. Devido a isto, a análise das modificações das propriedades dos mésons vetoriais é um dos métodos mais eficazes da atualidade para estudar as características do meio nuclear. As seções de choque de interação méson-nucleon podem ser estudadas pela análise das possíveis modificações nas propriedades dos mésons vetoriais. HERA encontrou também uma nova classe de processos hard: produção de mésons vetoriais leves para valores altos de virtualidade $Q^{2}$ e produção de mésons pesados em todo o intervalo de valores da virtualidade $[15,16]$. Devido a esta descoberta, análises combinadas de espalhamento inelástico profundo (DIS) da produção hard de mésons vetoriais poderiam dar maior esclarecimento o entendimento à interação forte em altas energias. Estes fenômenos são amplamente estudados nos colisores de partículas mencionados acima. 
As reações nucleares induzidas por fótons para energias do centro de massa acima de $W \sim 1 \mathrm{GeV}$ mostram um processo diferente dos processos que acontecem nas energias no intervalo das ressonâncias bariônicas [17]. Nas altas energias, o fóton apresenta uma componente hadrônica na sua função de onda que aumenta a seção de choque de interação com os nucleons. De acordo com o modelo de dominância vetorial (VMD) [18-21], no meio nuclear, a seção de choque total é dominada pela produção de estados ligados $(q \bar{q})^{J=1}$ conhecidos como mésons vetoriais. Estes estados ligados se propagam pela matéria nuclear interagindo com os nucleons no processo conhecido como interação no estado final (FSI).

Uma reação nuclear é geralmente descrita em duas etapas, uma representando a fase rápida, onde as interações hadrônicas mudam localmente a densidade nuclear, e a outra descreve o decaimento do sistema depois que este atingiu o equilíbrio térmico. A fase rápida leva cerca de $10^{-22} \mathrm{seg} .(\sim 30 \mathrm{fm} / \mathrm{c})$, onde o núcleo perde parte da energia incidente através de emissões diretas e de pré-equilíbrio evoluindo para um estado termalizado em equilíbrio chamado de núcleo composto (NC). Este processo é conhecido na literatura como cascata intranuclear. A segunda etapa tem uma duração de $10^{-17}$ seg. $\quad\left(\sim 3 \times 10^{6} \mathrm{fm} / \mathrm{c}\right)$ e evolui através da emissão de partículas ou pelo processo de fissão nuclear[22].

Devido ao nível de complexidade na descrição do processo o método de maior sucesso é o Monte Carlo, que considera a cascata intranuclear como uma sequência de eventos de partículas individuais. Estes modelos estão baseados no princípio de que na cascata intranuclear ocorrem apenas interações binárias, pois o tempo de interação é muito menor do que o tempo médio entre as colisões sucessivas, i. e.,

$$
\tau \ll \frac{d}{v} \quad \text { e } \quad \lambda \ll d
$$

onde $\tau$ é o tempo de interação, $d$ e $v$ são a distância e velocidade relativa entre as partículas e $\lambda$ é o comprimento da onda de De Broglie da dispersão. O comprimento de onda é suficientemente pequeno permitindo usar a aproximação de que as interações entre nucleons são independentes entre si, o que significa que o grau de dispersão de uma interação binária não será influenciada pela presença de nucleons externos a ela. O processo de dispersão depende dos efeitos de correlação binários na interação nucleon - nucleon na matéria nuclear. Assim, o comprimento de onda dos nucleons deve ser pequeno em relação ao alcance efetivo das interações nucleares $r_{\text {eff }}$ :

$$
\lambda \ll r_{e f f} \leq v \tau \ll d .
$$

A condição (1.2) limita a faixa de energia cinética das interações da cascata. De fato, os 
modelos de cascata só são válidos para energias cinéticas $\gtrsim 250 \mathrm{MeV}$. Alguns dos mais velhos e conhecidos códigos que usam esta abordagem para a cascata intranuclear e/ou processo de evaporação são o modelo de Bertini [23], o modelo da cascata intranuclear ISABEL (INC) [24, 25] e o modelo de cascata intranuclear de Barashenkov e colaboradores [26-29]. Estes modelos apresentam um modelo nuclear onde a matéria nuclear é dividida em regiões concêntricas com densidades diferentes. Nestas regiões os nucleons mostram uma distribuição de um gás Fermi. No entanto, a energia de Fermi é calculada usando uma aproximação de densidade local, ou seja, o momento e a energia de Fermi são dependentes do raio da região. Nos modelos ISABEL INC, a densidade nuclear tem 16 regiões, ao invés de três, como no código Bertini INC e sete na versão do código CASCADE. As interações entre hádrons estão dadas por parâmetros extraídos de dados experimentais de acordo à lei de conservação de energia-momento. CASCADE considera a diminuição da densidade intranuclear devido a um knock-out de nucleons já que este efeito é especialmente importante em altas energias e em colisões núcleo-núcleo num ambiente com muitas interações intranucleares [28].

Esses modelos antigos consideram a cascata intranuclear como uma sequência de eventos de partícula única baseada na hipótese pouco realista de que todas as outras partículas dentro do núcleo são objetos estáticos, assim cada ramificação da cascata é independente das demais. Esta hipótese não considera as colisões diretas entre partículas re-espalhadas fazendo com que alguns fenômenos físicos conhecidos como o bloqueio de Pauli e as flutuações de densidade locais não sejam eficazmente tratadas na simulação. A cascata intranuclear é inicializada por uma partícula incidente que colide com o núcleo e é interrompida quando todas as partículas envolvidas na cascata que tem possibilidade de sair do núcleo conseguem. Outro aspecto importante é a inclusão do princípio de exclusão de Pauli que é levado em conta aceitando apenas as colisões em que os nucleons resultantes tiverem uma energia maior que a energia de Fermi da região. O modelo de Bertini é baseado em uma abordagem independente do tempo que leva em conta as interações entre nucleons isolados no meio nuclear, enquanto no código ISABEL são eliminadas as colisões entre as partículas que resultam em núcleos com energia acima do nível de Fermi. Estes modelos clássicos têm a desvantagem de não permitirem seguir o curso dos núcleos durante o desenvolvimento da cascata, evitando assim obter informações de uma grandeza em qualquer instante de tempo no intervalo da evolução da cascata. Porém, muitas versões desses códigos foram desenvolvidas ao longo do tempo apresentando melhorias e obtendo resultados interessantes, alguns dos quais nos processos de fotoprodução [9, 30-39].

Com o desenvolvimento do poder de cálculo computacional novos modelos de cascata intranuclear foram propostos para tratar a cascata intranuclear usando uma abordagem multicolisional [40-43]. Este tipo de modelo proporciona uma ferramenta poderosa para 
o estudo de reações nucleares, porque trata a evolução temporal semiclássica de todas as partículas através de uma dinâmica de muitos corpos permitindo obter o valor de qualquer observável no transcurso da cascata. Uma discussão sobre a vantagem destes códigos em relação aos códigos mais antigos podem ser encontradas em Ref. [17, 44-46].

Uns dos códigos mais avançados que seguem esta abordagem multicolisional é o código INC Liege. Este foi primeiramente proposto para a descrição das colisões de íons pesados na faixa de energia dos GeV [47]. A partir da versão INCL2 [41] se introduz o estudo de reações nucleares induzidas por nucleons. Foi incluído também o princípio de exclusão de Pauli por meio de fatores estatísticos que dependem da probabilidade de ocupação no espaço de fase da vicindade das partículas que interagem. Esta probabilidade é dada pelo números de partículas que cabem em um volumem dado pelo produto de uma esfera de radio $r=2 \mathrm{fm}$, no espacio da posição, e uma esfera de radio $r=200 \mathrm{MeV} / \mathrm{c}$ no espacio dos momentos [41]. Este mecanismo leva a violações do princípio de Pauli que se verificam nos números de ocupação incorretos nos níveis de energias menos energéticos, na obtenção de energias negativas do núcleo residual excitado e no processo conhecido como nuclear boiling. Estas violações geram dificuldades, tais como a impossibilidade de manter o núcleo estável durante muito tempo mesmo sem ser excitado, incorreta excitação do núcleo residual e espectro de partículas secundárias e multiplicidade em desacordo com os dados experimentais. Outra versão deste código foi proposta, INCL4 [40]. Esta foi melhor sucedida na reprodução das colisões de muitos corpos com a introdução do que os autores chamam de determinação de um tempo de parada auto-consistente do processo em cascata. O modelo diferencia os nucleons participantes dos nucleons espectadores. Os participantes são aqueles nucleons que colidem com outros participantes, assim os primeiros participantes são os nucleons resultantes do mecanismo de interação inicial, enquanto que os espectadores não podem colidir com outros. Estas restrições foram impostas para evitar a emissão espontânea da esfera de Fermi (spontaneous boiling[40]), onde um nucleon que está perto da superfície de Fermi pode obter energia através de colisões com outros nucleons e escapar do núcleo. Esta versão introduz uma superfície nuclear difusa e bloqueia as colisões se a energia de excitação dentro da esfera de Fermi se tornasse negativa. Esta abordagem evita que algum nucleon tenha um momento menor do que o momento de Fermi e levando a uma incorreta deformação do mar do Fermi, causando assim a existência de um pequeno número de eventos com energia de excitação negativa. Em trabalhos mais recentes, um mecanismo rigoroso do princípio de exclusão de Pauli foi introduzido [48], onde são proibidas as colisões que resultam em nucleons com um momento menor que o momento de Fermi. Este mecanismo não leva em conta a deformação da esfera de Fermi. Os dados experimentais sugerem um mecanismo de exclusão de Pauli entre as duas variantes [48]. Uma comparação interessante entre este códigos num estudo de S. Leray, A. Boudard e J. Gugnon et. al. pode ser encontrada na 
refêrencia [49]. Este abordagem multicolisional, que inclui de forma natural as flutuações de densidade nuclear durante a cascata e também as variações do número de ocupação de cada nível energia para cada partícula como uma função do tempo, tem obtido bons resultados até a presente data [43, 49-51].

Outro código MC dependente do tempo que vale a pena mencionar é o código MCMC (Multicolisional Monte Carlo) [42]. O código de MCMC foi inicialmente desenvolvido para estudar colisões de íons pesados relativísticos e também é utilizado para descrever fotorreações em altas energias $(0,5-1,5 \mathrm{GeV})[42,52]$. MCMC foi o primeiro software a incluir píons e ressonâncias, especificamente as ressonâncias $\Delta$, na dinâmica multicolisional da cascata intranuclear e não como uma superposição das trajetórias de individuais destas. Esta abordagem tem um efeito direto na multiplicidade das partículas emitidas e na energia de excitação do núcleo residual. No entanto, um dos efeitos mais importantes a incluir no modelo INC, o efeito de Pauli, ainda é incluído da mesma forma que nos modelos convencionais. Por outro lado, o processo de competição entre fissão e evaporação que segue depois da cascata no decaimento do núcleo residual foi tratado na versão mais simples desde que apenas a evaporação de nêutrons é relevante, em comparação com o proceso de fissão. No entanto, trabalhos posteriores demonstraram que esta hipótese não está correta [53] mesmo para núcleos pesados, para o quais a fissilidade está perto de 100 \%. O código MCEF (Monte Carlo Evaporação e Fissão [53, 54]) soluciona este problema pela inclusão, no processo de competição evaporação/fissão da evaporação de prótons e partículas alfa. Com isso, é possível prever, com boa precisão, os valores de fissilidade de actinídeos e preactinídeos [55]. Estes códigos têm obtido bons resultados até agora em vários trabalhos [56-63].

O código CRISP (Colaboração Rio - São Paulo) [17] é um software em C++ que tem sido desenvolvido nos últimos 25 anos. Este propõe o acoplamento entre os modelos usados nos códigos MCMC e MCEF (Monte Carlo Evaporação e Fissão [53, 54]) na mesma rotina. O CRISP interpreta corretamente o comportamento médio de algumas grandezas em processos de fotorreações a energias intermediárias e altas. O código apresenta melhorias significativas se comparado com códigos similares: i) A exitação de ressonâncias mais pesadas que as ressonâncias $\Delta$, ii) um estado fundamental nuclear utilizando o modelo de gás de Fermi e o principio de exclusão de Pauli, iii) um mecanismo de exclusão de Pauli mais realista, $i v$ ) o canal de quase-deuteron na fotoprodução e $v$ ) um critério de parada da cascata livre de parâmetros e baseado estritamente em critérios energéticos. A diferença fundamental do código em relação às outras versões é a dinâmica temporal total em que se desenvolve a cascata intranuclear [42, 64]. Esta dinâmica é considerada num núcleo com um potencial quadrado e inclui todas as partículas deste. As partículas participam em colisões binárias, reflexões na superfície e a emissão na fase de pré-equilíbrio. Os efeitos do potencial nuclear se manifestam na transmissão das 
partículas através da superfície nuclear pela aproximação da massa efetiva de acordo com a aproximação do campo médio de Wallecka [65]. Ao longo da simulação multicolisional da cascata intranuclear se atualizam todas as variáveis cinemáticas das partículas dentro do núcleo que permite a descrição mais realista de vários fenômenos.

Este modelo multicolisional do código CRISP é um dos mais sofisticados em termos de modelos de transporte porque o destino do processo do sistema nuclear está completamente determinado pela condição inicial (interação inicial e o estado básico do núcleo antes da colisão) e não depende de parâmetro nenhum, exceto aqueles dos modelos usados nas colisões entre partćulas. O diagrama 1.1 mostra a evolução temporal de um processo de cascata multicolisional que inclui interação inicial, emissões diretas de préequilíbrio e processos de reflexão na superfície nuclear. Estes processos, assim como o processo de interação elementar, o movimento fermiônico e o mecanismo de bloqueio de Pauli serão explicados em detalhe no capítulo 2.

A descrição semiclássica de um mecanismo de reação para múltiplas colisões binárias no espaço de configuração é complicada pelo tratamento de efeitos quânticos causados pelas correlações nucleon-nucleon e pelo fato de que as distribuições de energia e de impulso não obedecem regras de seleção e nem discretização dos níveis previstos pela mecânica quântica. Uma violação deste tipo conduz à ocupação incorreta dos estados quânticos fisicamente inacessíveis ao sistema. Um destes efeitos quânticos é o bloqueio de Pauli. Todo processo da cascata intranuclear simulado no código CRISP é dominado pelo mecanismo de bloqueio de Pauli que resulta da simetrização da função de onda do nucleon no ambiente nuclear. Sua principal consequência é a proibição de processos no meio nuclear que seriam permitidos no vácuo. O modelo desenvolvido no código CRISP difere dos códigos similares em vários aspectos, uma vez que incorpora um tratamento realista do mecanismo de bloqueio de Pauli baseado numa estrutura de camadas no espaço dos momentos e o princípio de incerteza de Heisenberg. O mecanismo determina o comportamento no processo de fotoabsorção inicial, de cada interação binária nucleon-nucleon e cada desintegração ou formação de ressonâncias no meio nuclear sem incluir nenhum parâmetro livre. O CRISP, ao mesmo tempo, incorpora uma condição de equilíbrio térmico como condição para a parada da cascata. Os resultados obtidos mostram a consistência desta nova abordagem que evita as deficiências e desvantagens em outros modelos desenvolvidos até a presente data.

O código CRISP tem obtido bons resultados no estudo de reações induzidas por prótons [45, 46, 66, 67], fótons [17, 44, 55, 68], elétrons [69, 70] e processos que dependem da cascata intranuclear, como a produção de káon [71] e o decaimento de hypernúcleos [7274]. Os processos de evaporação/fissão estudados com o código obtiveram pela primeira 
vez o efeito de saturação abaixo da unidade de fissilidade observado nos experimentos com núcleos pesados $[53,54,75]$.

$\mathrm{Na}$ atualidade existe um grande interesse nos fenômenos nas altas energias porque oferecem o único método para estudar e compreender as propriedades da matéria nuclear quente e densa. Para isso é preciso o estudo da equação do estado nuclear (EoS) e da seção de choque de interação nucleon-nucleon num meio com estas características. Um grande número de observáveis foram propostos e/ou medidos durante as três últimas décadas, por exemplo o fluxo plano transversal [76], o fluxo elíptico [77, 78], a energia de equilibrio [79-82], a energia de transição [78, 83, 84], isospin tracing [85, 86], multifragmentação [87, 88], distribuição de rapidity [89, 90] etc. Várias destas grandezas são estudadas atualmente nos experimentos realizados nos colisores LHC (The Large Hadron Collider) e no JLab (Thomas Jefferson Accelerator Facility).

Em uma reação nuclear em altas energias é produzida uma grande quantidade de partícula carregadas e neutras e os fenômenos tem uma alta multiplicidade. Por exemplo, um evento típico no LHC consiste na produção de cerca de 100 partículas carregadas e outras tantas neutras. À luminosidade nominal do LHC, cada 15 ns são produzidos 20 desses eventos aproximadamente. Ao mesmo tempo, muitos sinais fazem uso de propriedades coletivas destas partículas, tais como jatos e o momentum transversal de neutrinos e outras partículas não detectadas. Outros sinais dependem de partículas individuais tais como elétrons, múons ou fótons e precisam ser detectadas com algum grau de certeza. Os sinais destas partículas vem frequentemente acompanhados de um forte background produzindo flutuações que atrapalham a medição. Devido a isto o Monte Carlo é o método mais poderoso à nossa disposição para obter uma compreensão detalhada e realista da física em altas energias. Uma correta simulação MC deve incluir efeitos nucleares e individuais como os diferentes processos de interação das partículas a partir das funções de estrutura medidas e parametrizadas previamente de modelos teóricos de pártons, eventos similares, processos de fragmentação etc. O CRISP é um código deste tipo que, incluindo corretamente as interações mais relevantes no intervalo de energias onde os processos estão sendo analisados, poderia ser uma boa ferramenta para o estudo destes e para fazer novas previsões. Aliás, seus resultados poderão ser usados como uma ajuda para o planejamento de um novo detector e para a elaboração das estratégias de análise de dados reais e a otimização das condições de sinal-fundo.

Por consequência, o seguinte problema surge:

"Como simular reações nucleares induzidas por fótons nas altas energias usando o método de Monte Carlo implementado no código CRISP?"

As reações nucleares induzidas por fótons para energias do centro de massa acima de $W \sim 1 \mathrm{GeV}$ mostram um processo diferente dos processos que acontecem nas energias no 


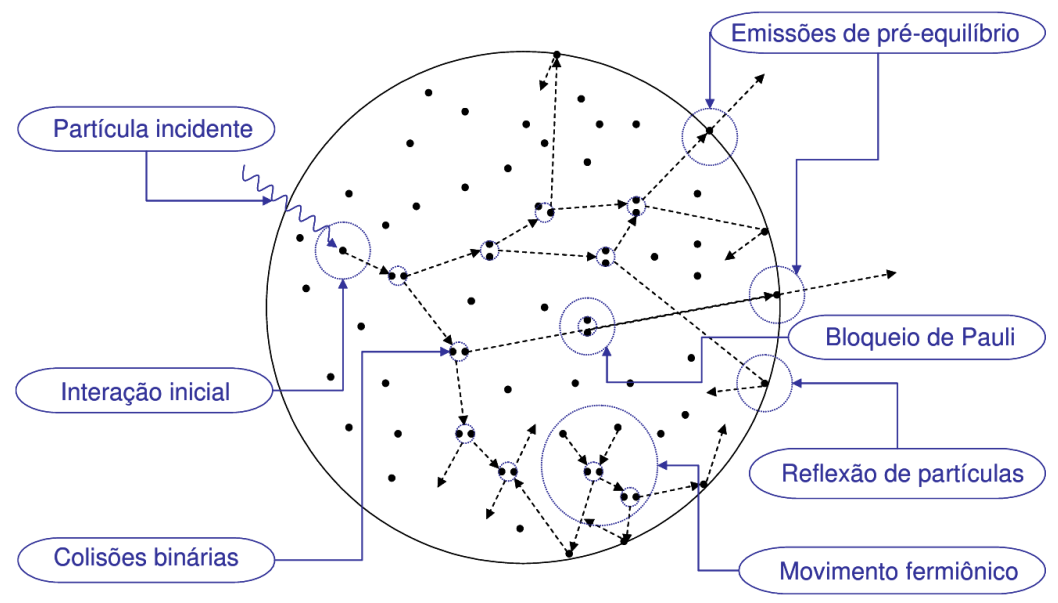

FigurA 1.1: Representação do mecanismo de cascata intranuclear. Figura extraída da Ref. [22].

intervalo das ressonâncias bariônicas [17]. A altas energias, o fóton apresenta uma componente hadrônica na sua função de onda que aumenta a seção de choque de interação com os nucleons. De acordo com o modelo de dominância vetorial (VMD) [18, 19], no meio nuclear, a seção de choque total resulta fundamentalmente na produção de estados ligados $(q \bar{q})^{J=1}$ conhecidos como mésons vetoriais. Estas partículas são muito importantes já que analisando as modificações de suas propriedades, podem ser estudadas as características do meio nuclear. O experimento HERA encontrou uma nova classe de processos hard: produção de mésons vetoriais leves para valores altos de virtualidade $Q^{2}$ e produção de mésons pesados em todo o intervalo de valores da virtualidade $[15,16]$. Devido à estas descobertas, análises combinadas de espalhamento inelástico profundo (DIS) de produção hard de mésons vetoriais esclareceriam o entendimento da interação forte a altas energias.

O problema poderia ser resolvido a partir da seguinte hipótese:

Se é assumido que: $i$ ) os fótons nas altas energias se comportam como um hádron de acordo ao modelo de dominância vetorial, $i i$ ) o modelo multicolisional de cascata intranuclear é uma boa ferramenta para descrever as reações nucleares que incluam interações hádron-hádron e iii) o código CRISP consegue obter bons resultados no intervalo das energias das ressonâncias bariônicas, então este código poderá simular reações fotonucleares em altas energias se fora incluída a produção de mésons vetoriais e uma correta interação no estado final destas no ambiente nuclear.

De acordo com o parágrafo anterior é definido o seguinte objetivo:

Expandir o código CRISP pela inclusão do processo de fotoproducção de mésons vetoriais e a interação destes com o núcleo. 
Este trabalho estuda reações nucleares induzidas por fótons no intervalo das médias e altas energias desde o limiar de produção dos mésons vetoriais até vários $G e V$ s usando o modelo de cascata intranuclear desenvolvido no código CRISP. Os mésons vetoriais que serão incluídos são $\rho, \omega, \phi$ e $\mathrm{J} / \Psi$. Em altas energias a interação no estado final dos mésons vetoriais pode ser substituída pelo conjunto de interações binárias entre os méson e o hádrons. Para alcançar o objetivo principal é necessário definir vários objetivos específicos:

- Fazer um estudo na literatura dos canais de produção de mésons vetoriais em fotorreações na faixa de médias e alta energias.

- Fazer um estudo na literatura dos canais de interação de mésons vetoriais com o meio nuclear na faixa de médias e alta energias.

- Implementar no código CRISP os canais relacionados com a produção dos mésons vetoriais $\rho, \omega, \phi$ e $\mathrm{J} / \Psi$

- Implementar no código CRISP os canais interação no estado final dos mésons vetoriais e o núcleo.

- Verificar a inclusão dos novos canais pela simulação da fotoabsorção e da interação dos mésons vetoriais com prótons e comparar com dados experimentais.

- Simular processos nucleares na faixa de médias e alta energias.

- Calcular grandezas de interesse nos processo fotonucleares em altas energias e comparar com dados experimentais.

A cascata intranuclear que se formará será processada de acordo com o modelo já utilizado no código incluíndo o efeito do potencial nuclear para os nucleons, o mecanismo de Pauli nas colisões méson- nucleon e as emissões pré-equilíbrio dos mésons. O resultado desta cascata será a formação de um núcleo residual que será modificado de acordo com a competição entre os processos de evaporação e fissão nuclear. Com isso o CRISP poderá ser utilizado para investigar a atenuação de hádrons no núcleo, efeitos nucleares sobre o processo de produção e multiplicidade de hádrons secundários produzidos e seus espectros. Além disso, todas as características do núcleo residual formado ao final da reação poderão ser determinadas. Uma aplicação de grande importância são as colisões ultraperiféricas no LHC (The Large Hadron Collider) as quais serão introduzidas aqui já que para fazer um estudo correto deste fenômeno é preciso uma simulação realista da dinâmica da cascata que inclua os processos de fotoprodução de mésons vetoriais.

A tese está organizada da seguinte maneira: no capitulo 2 é explicado o modelo de cascata intranuclear por Monte Carlo desenvolvido no CRISP, no capítulo 3 são estudadas 
as colisões de partículas em altas energias e o processo de colisões ultraperiféricas, no capítulo 4 é feita uma descrição dos processos que serão adicionados no código, relacionados com a produção e interação com o meio nuclear dos mésons vetoriais. No capítulo 5 são mostrados os resultados do trabalho em cada etapa e comparados com os dados experimentais. No capítulo 6 serão expostas as conclusões do trabalho até a presente data e apresentados os próximos passos a seguir para complementar a pesquisa. 


\section{Capítulo 2}

\section{O Modelo de Cascata}

\section{Intranuclear e Evaporação/Fissão}

Neste capítulo serão descritos a teoria e o modelo de cascata intranuclear e o processo de evaporação e/ou fissão nuclear empregado no código CRISP. As principais características que diferenciam o CRISP em relação a outros códigos existentes serão discutidas em detalhes, evidenciando as principais melhorias e modificações introduzidas para obter uma adequada descrição do ambiente nuclear para que suas propriedades sejam satisfatoriamente reproduzidas na evolução temporal de um sistema nuclear complexo e excitado.

\subsection{Modelo da cascata intranuclear CRISP}

Uma descrição correta da fase rápida de uma reação nuclear nas energias intermediárias e altas ( $\gtrsim 50 \mathrm{MeV}$ ) está baseada na seguinte abordagem: inicialmente um mecanismo de interação inicial produz uma reação inicial que transfere energia a um ou vários nucleons do núcleo que interagem com os outros nucleons através de interações binárias. Com a evolução do sistema partícula inicial-núcleo o número de partículas envolvidos nesta dinâmica multicolisional aumentará, distribuíndo a energia em todo o núcleo. O tipo de partículas participantes no processo varia com a energia; nas médias energias, as partículas que participam no processo são nucleons, ressonâncias e píons, enquanto que em altas energias, mésons leves e pesados são criados dentro do núcleo. Vários efeitos nucleares são levados em conta para uma correta descrição do processo como são as emissões diretas de pre-equilíbrio, e do processo de desintegração do núcleo composto. 
Chapter 2. O modelo de cascata intranuclear e Evaporação/Fissão usando o método de

\subsubsection{O estado fundamental}

O estado fundamental é sempre gerado a partir de uma distribuição de momento usando o modelo de um gás de Fermi uniforme incluindo os graus de liberdade associados ao spin. Com eles espera-se reproduzir a média dos efeitos quânticos no meio nuclear.

\subsubsection{Distribução da posição e dos momentos dos nucleons}

Nesta seção é descrita a distribuição de momentos dos núcleos no estado nuclear fundamental. Inicialmente a distribuição inicial dos momentos dos nucleons está de acordo com uma distribuição de Fermi onde os estados das partículas $|p\rangle$ correspondem aos estados de onda plana do gás de Fermi que não interagem entre si, i.e.:

$$
\hat{H}|p\rangle=\epsilon|p\rangle
$$

onde $H$ é o operador de Hamilton, e $\epsilon$ as energias correspondentes ao estado; o operador de Hamilton pode ser reescrito na forma relativística:

$$
H=\sqrt{p^{2}+m_{0}^{2}}
$$

onde $p$ é o momento do nucleon e $m_{0}=938 \mathrm{MeV}$ é a sua massa de repouso ${ }^{1}$. O espaço de fase dos momentos está dividido em células cujo volume está definido de acordo ao modelo de gás de Fermi e o princípio de incerteza de Heisenberg. De acordo ao modelo, a energia de Fermi para nêutrons e prótons é

$$
\begin{gathered}
\epsilon_{F}^{p}=\frac{1}{2 m_{0}}\left(3 \pi^{2}\right)^{\frac{2}{3}}\left(\frac{Z}{\Omega}\right)^{\frac{2}{3}} \\
\epsilon_{F}^{n}=\frac{1}{2 m_{0}}\left(3 \pi^{2}\right)^{\frac{2}{3}}\left(\frac{A-Z}{\Omega}\right)^{\frac{2}{3}} .
\end{gathered}
$$

Estas células de momento no espaço de fase dos momentos estão agrupadas em camadas concêntricas com volume $4 \pi p^{2} \Delta p$ onde $p$ é o valor do momento dos nucleons e $\Delta p$ é um intervalo do momento que é calculado na base do princípio de incerteza de Heisenberg:

$$
\Delta p \sim \frac{\hbar}{2 \Delta r}
$$

\footnotetext{
${ }^{1}$ No código CRISP é usado o sistema de unidades naturais onde $c=\hbar=1$
} 
Chapter 2. O modelo de cascata intranuclear e Evaporação/Fissão usando o método de

onde $\Delta r$ é a incerteza da posição do nucleon e.g., para o núcleo pesado ${ }^{208} \mathrm{~Pb}$ temos $\Delta r \sim 7.0 \mathrm{fm}$ e $\Delta p \sim 14 \mathrm{MeV} / \mathrm{c}$. O número de ocupação para cada nível está definido de

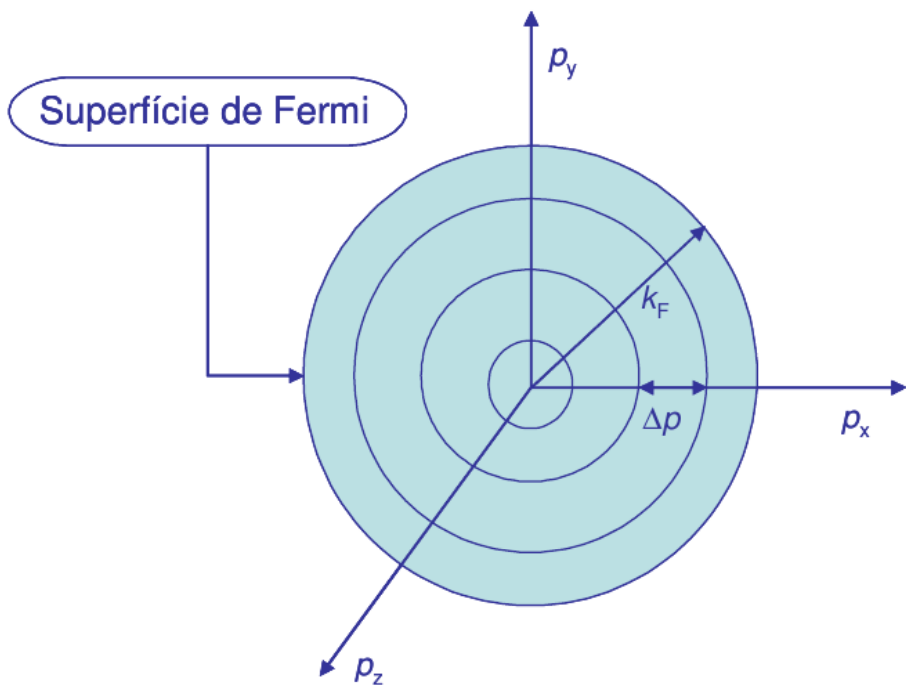

FIGURA 2.1: Estrutura em camadas no espaço dos momentos para simular o mecanismo de Pauli [22].

acordo aos número quânticos do nucleons. No CRISP, estes estão confinados num poço de potencial infinito quadrado de comprimento L onde os autovetores para $i=x, y, z$ :

$$
\Psi= \begin{cases}0 & \text { se } x_{i}<0, \\ \sqrt{\frac{2}{L}} \sin \left(\frac{\pi n}{L} x_{i}\right), n_{i} \in N^{+} & \text {se } 0<x<L, \\ 0 & \text { se } x_{i}>L\end{cases}
$$

têm autovalores:

$$
E_{n}=\frac{\hbar^{2}}{2 m L^{2}} n^{2}, n \in \mathbf{N}^{+}
$$

Assim, os níveis quânticos são caracterizados pelas componentes $p_{i}=n_{i} \Delta p$ com $n_{i}$ inteiro. Portanto, o momento total é dado por

$$
p^{2}=p_{x}^{2}+p_{y}^{2}+p_{z}^{2}=\left(n_{x}^{2}+n_{y}^{2}+n_{z}^{2}\right)(\Delta p)^{2}=n^{2}(\Delta p)^{2}
$$

com $n$ inteiro. A condição $n$ e $n_{i}$ inteiros restringe o número de estados possíveis. Os números quânticos possíveis são caracterizados por números inteiros $n_{x}, n_{y}, n_{z}$ que 
Chapter 2. O modelo de cascata intranuclear e Evaporação/Fissão usando o método de

satisfazem a equação 2.8. Por exemplo para $n=1$ são só possíveis três combinações:

$$
\begin{aligned}
& n_{1}\left(n_{x}, n_{y}, n_{z}\right)=(1,0,0) \\
& n_{2}\left(n_{x}, n_{y}, n_{z}\right)=(0,1,0) \\
& n_{3}\left(n_{x}, n_{y}, n_{z}\right)=(0,0,1)
\end{aligned}
$$

que multiplicado pelo grau de liberdade do spin resulta em 6 estados prováveis para o primeiro nível dos prótons e 6 para os nêutrons.

Os núcleons estão confinados no volume esférico $\Omega=\frac{4}{3} \pi r_{0}^{3} A$ e não há restrições do ponto de vista quântico para as posições dos nucleons no núcleo durante a cascata.

Esta distribuição é uma das características do CRISP que o diferencia dos códigos anteriores, onde os nucleons foram distribuídos uniformemente para um valor médio de energia de Fermi entre prótons e nêutrons. As últimas versões do código CRISP levam em conta a assimetria no espaço dos momentos causadas pelo excesso de nêutrons em núcleos pesados. As equações (2.3) e (2.4) medidas a partir da parte inferior do poço dos prótons e nêutrons, respectivamente, permitem que a energia de Fermi no sistema de referência de laboratório seja a mesma para prótons e nêutrons pois, isto evita que o núcleo em estado de repouso decaia via decaimento $\beta$, como é esperado no comportamento de um núcleo real.

Para os nêutrons os estados fisicamente acessíveis são distribuídos uniformemente num espaço de momentos entre $k_{n}^{\text {min }}=0$ e $k_{n}^{\text {max }}=k_{F}^{n}$, enquanto os prótons são distribuídos $\operatorname{com} k_{p}^{\text {min }}=k_{F}^{n}-k_{F}^{p}$ e $k_{p}^{\text {max }}=k_{F}^{n}$. Então usando as variável pseudoaleatórias $x_{1}, x_{2}$ e $x_{3}$ uniformemente distribuídas no intervalo [0..1] é possível escrever $\theta_{p} \varphi_{p}$ usando as formas de inversão:

$$
\begin{aligned}
\varphi_{p} & =2 \pi x_{1} \\
\theta_{p} & =\arccos \left(1-2 x_{2}\right) \\
p & =k_{F}^{n} x_{3}^{\frac{1}{3}} \text { (para nêutrons) } \\
e & \\
p & =\left[\left(\left(k_{F}^{n}\right)^{3}+\left(k_{\text {min }}^{p}\right)^{3}\right) x_{3}+\left(k_{\text {min }}^{p}\right)^{3}\right]^{\frac{1}{3}} \text { para prótons }
\end{aligned}
$$


Chapter 2. O modelo de cascata intranuclear e Evaporação/Fissão usando o método de

\subsubsection{Potencial nuclear e barreira de Coulomb}

O modelo de cascata intranuclear CRISP, de forma similar a outros modelos, está baseado na hipótese de que o potencial nuclear é constante no espaço e no tempo, assim as trajetórias dos nucleons são retilíneas entre duas interações consecutivas. Embora o potencial de Wood-Saxon descreva melhor a distribuição da densidade nuclear experimental, ele apresenta a desvantagem prática que a solução da equação de Schrödinger não resulta numa função analítica. Para núcleos pesados este potencial pode ser substituído em aproximação pelo poço quadrado. Assim este último vira um excelente candidato para uma boa descrição da dinâmica da cascata intranuclear. É importante notar nesta introdução, que esta abordagem do potencial quadrado tem pouca influência sobre os efeitos quânticos do mecanismo de exclusão de Pauli. Nos mecanismos de excitação nucleares, a aproximação de um potencial nuclear estático constante, que é o ponto de partida do modelo de cascata, é bastante razoável, tendo em conta que o campo nuclear médio não deve afetar significativamente as emissões durante o pré-equilíbrio.

Enquanto o processo de interação inicial e a dinâmica da cascata leva em conta distribuições realistas, o potencial usado para o efeito de confinamento é um poço quadrado como é mostrado na figura 2.2. O potencial nuclear é da forma:

$$
V=V_{0}=\epsilon_{F}+B
$$

onde $B$ é a energia de ligação que é determinada a partir de dados experimentais e é aproximadamente $\sim 7 \mathrm{MeV}$. No processo de emissão da cascata, os nucleons deverão ter uma energia suficiente para ultrapassar o valor deste potencial. Para o caso dos prótons, a energia deverá ultrapassar uma energia adicional que é a energia do potencial coulombiano como também é mostrado na figura 2.2. A altura da barreira de Coulomb $V_{C}$ é calculada sobre a superfície nuclear com $r_{\max }=r_{0} A^{1 / 3}$ e seu valor é:

$$
V_{C}=\frac{e^{2} Z_{1} Z_{2}}{r_{\max }}
$$

onde $Z_{1}$ e $Z_{2}$ são as cargas das partículas respectivamente com $e^{2}=1.44 \mathrm{MeV} / \mathrm{fm}$.

No CRISP também é considerado o efeito de tunelamento através da barreira Coulombiana. A probabilidade de transmissão deste efeito $T_{C}$ é calculada de acordo com a aproximação WKB:

$$
T_{C}=1 \quad \text { para } \quad T_{0}^{c i n}>V_{C}
$$

$\mathrm{e}$

$$
T_{C}=e^{-\frac{2}{\hbar}} \int_{r_{1}}^{r_{\max }} \sqrt{2 m\left(\frac{e^{2} Z_{1} Z_{2}}{r}-E\right)} d r \quad T_{0}^{c i n}<V_{C}
$$


Chapter 2. O modelo de cascata intranuclear e Evaporação/Fissão usando o método de

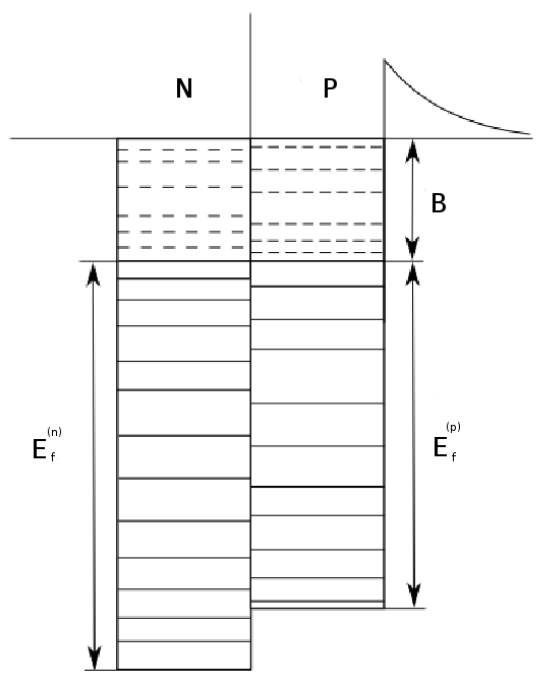

Figura 2.2: Diagrama do potencial nuclear no modelo de gás de Fermi

onde $T_{0}^{c i n}$ é a energia cinética assintótica $(r \rightarrow \infty)$ da partícula emitida e $r_{1}=\frac{e^{2} Z_{1} Z_{2}}{E}$ é o valor clássico de retorno. Calculando a integral, é obtida a fórmula de Gamow:

$$
T_{C}=\exp \left[\frac{-2 e^{2} Z_{1} Z_{2}}{\hbar v}(2 W-\sin (2 W))\right]
$$

onde $v=\sqrt{\frac{2 E}{m}}$ e $W=\arccos \left(\sqrt{\frac{r_{\max }}{r_{1}}}\right)$. No CRISP, o efeito de tunelamento permite a emissão de partículas com energia cinética insuficiente para ultrapassar a barreira de acordo a probabilidade calculada acima.

O potencial 2.15 impõe outra restrição ao gap de energia entre as camadas no espaço de momentos $\Delta p$. Por exemplo para o núcleo ${ }^{208} \mathrm{~Pb}$ obtém-se $\Delta p \approx 28.57 \mathrm{MeV} / \mathrm{c}$ para pótons, entanto para nêutrons $\Delta p \approx 26.45 \mathrm{MeV} / \mathrm{c}$. Usando o princípio de Heisenberg, este valor corresponde a $\Delta x \approx 5.5 \mathrm{fm}$ que é compatível com a dimensão de núcleo. Isto é uma clara indicação da auto consistência do modelo.

\subsubsection{O efeito do potencial nuclear. Massa efetiva dos nucleons ligados}

O Hamiltoniano efetivo dos nucleons ligados pode ser escrito como:

$$
H=T+V=\sqrt{p^{2}+m_{0}^{2}}-V_{0}
$$

Devido à complexidade algébrica para tratar a raiz quadrada, é conveniente a definição de um novo operador:

$$
H^{\prime}=\sqrt{p^{2}+\left(m_{0}-S\right)^{2}}
$$


Chapter 2. O modelo de cascata intranuclear e Evaporação/Fissão usando o método de

onde $S$ é um potencial escalar a ser determinado. Se $H=H^{\prime}$, é definida a grandeza $m^{*}=m_{0}-S:$

$$
\sqrt{p^{2}+m_{0}^{2}}-V_{0} \equiv \sqrt{p^{2}+m^{* 2}}
$$

A grandeza $m^{*}$ substitui assim a massa dos nucleons numa massa efetiva dos nucleons ligados. O problema se reduz a resolver o problema do nucleon livre com o valor da massa reduzida, isto é, o efeito dos potenciais nucleares correspondentes para uma diminuição da massa dos nucleons na matéria nuclear. A solução imediata da massa reduzida é

$$
m^{*}(p)=\sqrt{m_{0}^{2}+V_{0}^{2}-2 V_{0} \sqrt{p^{2}+m_{0}^{2}}}
$$

mostrando que $m^{*}$ é uma função do momento total do nucleon. No entanto, a solução exata não tem muita relevância prática devido à aproximação da poço quadrado. Fazendo o calculo para o ${ }^{208} \mathrm{~Pb}$ o valor médio da massa efetiva é $\left\langle m^{*}\right\rangle=0.950 m_{0}$, que está de acordo com os dados experimentais $\left\langle m^{*}\right\rangle=(0.953 \pm 0.002) m_{0}[91]$.

\subsubsection{O mecanismo de bloqueio de Pauli}

Um dos aspectos mais importantes na simulação da cascata intranuclear é a inclusão dos fenômenos quânticos, como o princípio de exclusão de Pauli. Esta questão foi abordada várias vezes em outros modelos atuais de cascata intranuclear. A metodologia utilizada no CRISP é um tratamento dos níveis de energia dos nucleons de uma forma não estocástica. O estado do núcleo pode ser representado por

$$
|\Psi\rangle=\left|p_{1}, p_{2} \ldots p_{N}\right\rangle
$$

onde $p_{1}, p_{2} \ldots p_{N}$ são os momentos das $\mathrm{N}$ partículas confinadas no ambiente nuclear. Em uma colisão binária, devido à característica desta interação, o estado das duas partículas e do núcleo formado pelo resto dos nucleons pode ser expresso na forma:

$$
|\Psi\rangle=\left|p_{1}, p_{2} \ldots p_{i-1}, p_{i+1} \ldots p_{j-1}, p_{j+1} \ldots p_{N}\right\rangle \otimes\left|p_{i}, p_{j}\right\rangle
$$

onde as partículas $i$ e $j$ representam as duas partículas que colidem. Então, a probabilidade de transição entre o estado $\left|p_{i}, p_{j}\right\rangle$ e o estado $\left|p_{k}, p_{l}\right\rangle$ vai estar determinada pelo termo matricial $\left\langle p_{k}, p_{l} \mid p_{i}, p_{j}\right\rangle$, que somente será diferente de zero se o princípio de exclusão de Pauli não for violado. Assim o espaço de fase dos nucleons é reduzido pela presença da materia nuclear.

O princípio de exclusão de Pauli está incorporado na cascata intranuclear fazendo uso da estrutura de camadas nucleares simetricamente esféricas no espaço dos momentos dos 
Chapter 2. O modelo de cascata intranuclear e Evaporação/Fissão usando o método de

nucleons. Esta abordagem implica que o número de prótons ou nêutrons com valor de momento entre $p$ e $p+\Delta p$ não excederá o número de máximo permitido para a camada associada a este valor do momento. No CRISP, o mecanismo de bloqueio Pauli consiste na verificação, depois de cada uma possível interação entre diferentes partículas ou um processo de decaimento no núcleo, se houver células disponíveis para o momento dos nucleons obtidos no estado final da reação. Se todos os nucleons secundários poderem ser corretamente colocados em níveis, a interação é permitida, caso contrário ela será bloqueada. Este processo regula todo processo nas fases da cascata, e também na construção do estado fundamental inicial de núcleo. Quando não há bloqueio na colisão o número de ocupação de cada camada é atualizado.

A confiabilidade do mecanismo de Pauli aqui proposto pode ser comprovada quando são analisadas as figuras 2.3, 2.4, 2.5, 2.6, onde são representados os níveis de ocupação do núcleo para diferentes momentos na cascata e comparados com o ajuste da função de partição à temperatura nuclear.
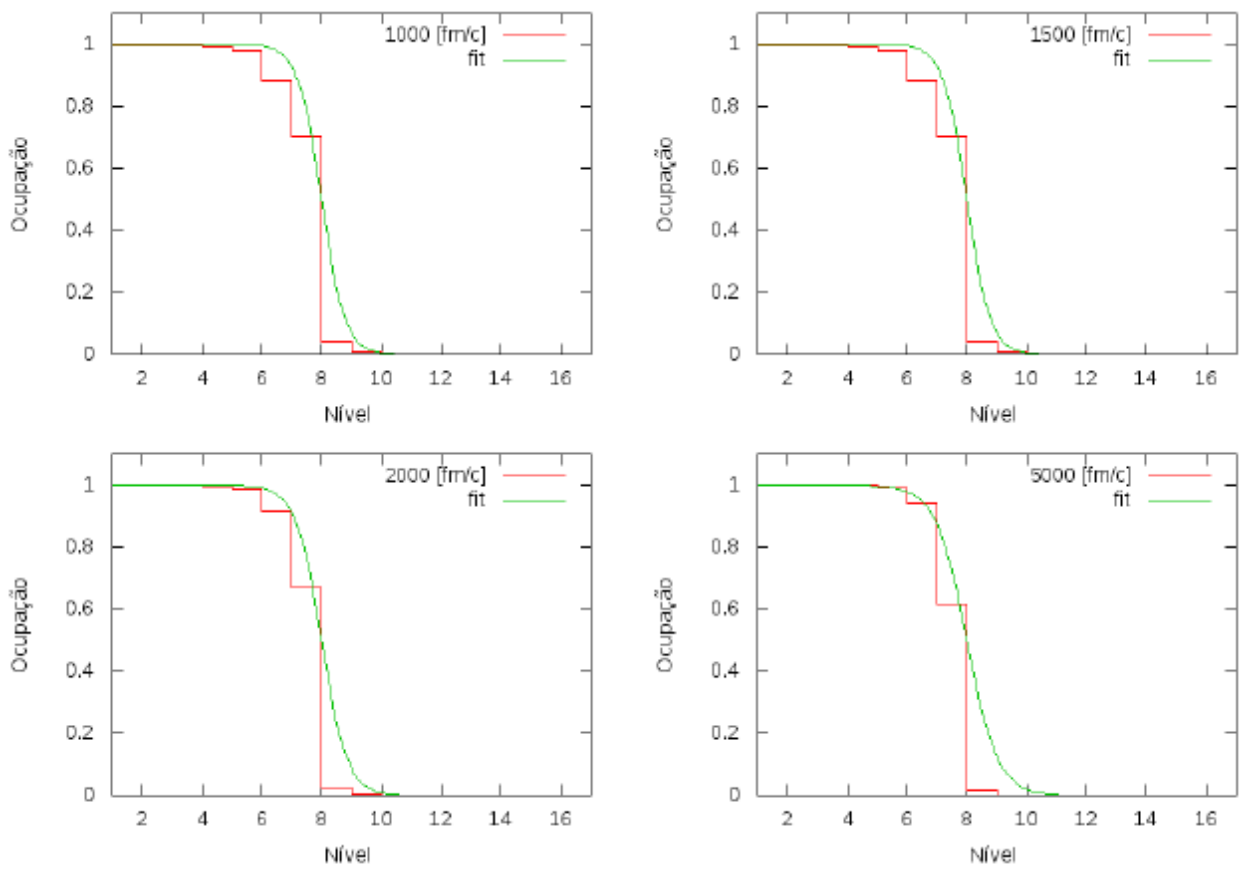

Figura 2.3: Distribuição do número de ocupação para prótons para vários instantes da cascata comparado com o ajuste da função de partição na temperatura. Figura obtida da Ref. [92] 
Chapter 2. O modelo de cascata intranuclear e Evaporação/Fissão usando o método de

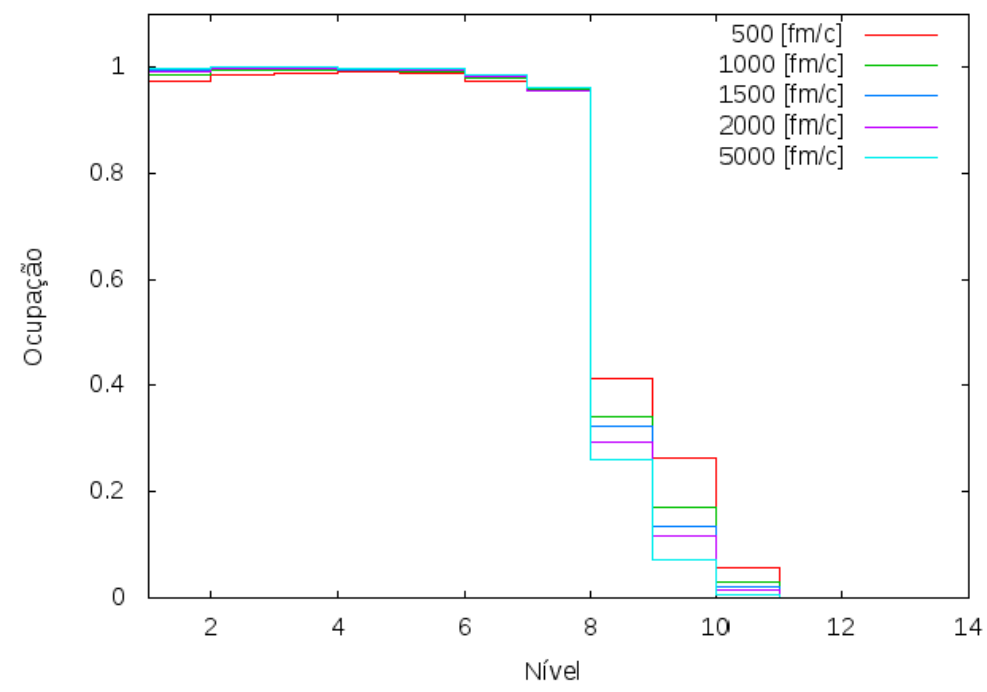

Figura 2.4: Distribuição do número de ocupação para prótons para vários instantes da cascata. Figura obtida da Ref. [92].
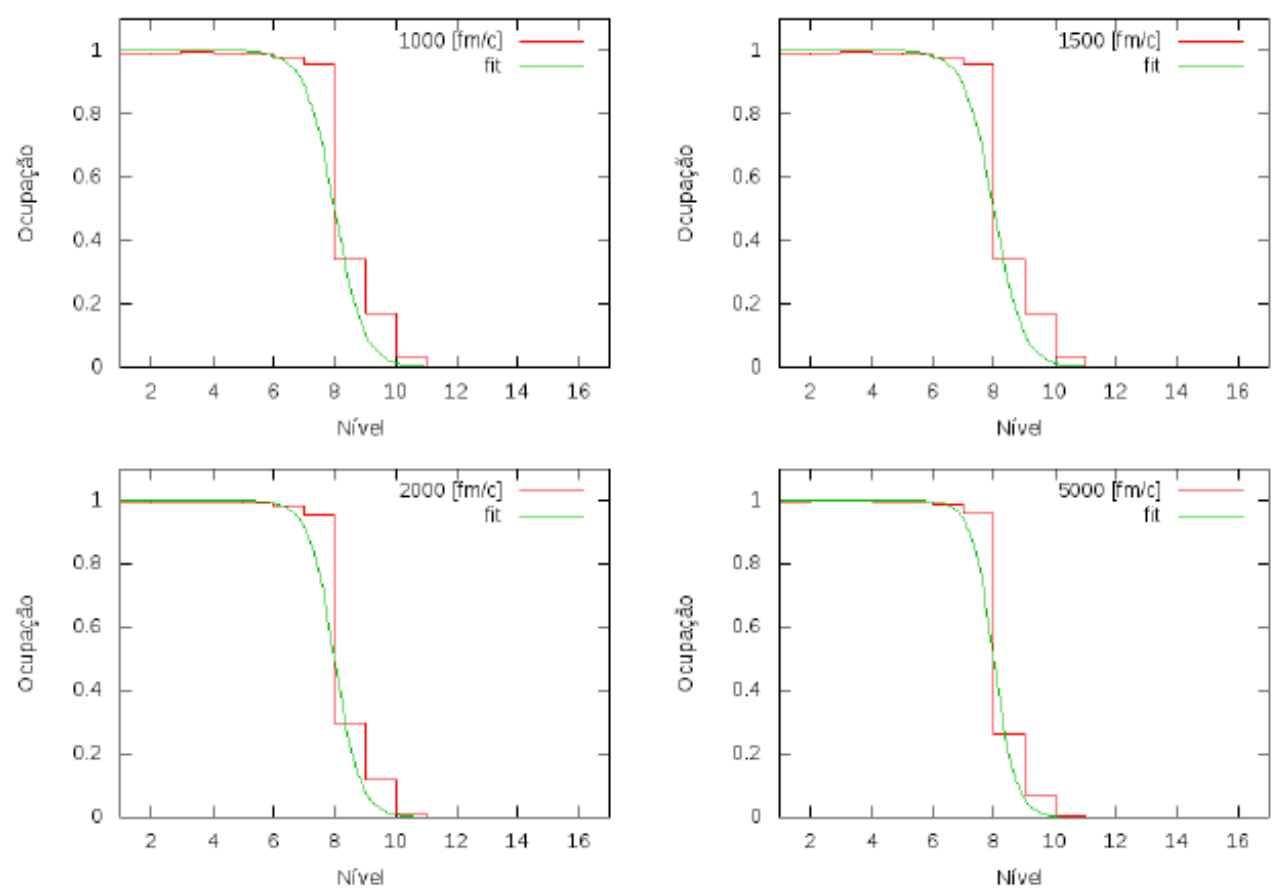

FIgURA 2.5: Distribuição do número de ocupação para nêutrons para vários instantes da cascata comparado com o ajuste da função de partição na temperatura. Figura obtida da Ref. [92]. 
Chapter 2. O modelo de cascata intranuclear e Evaporação/Fissão usando o método de

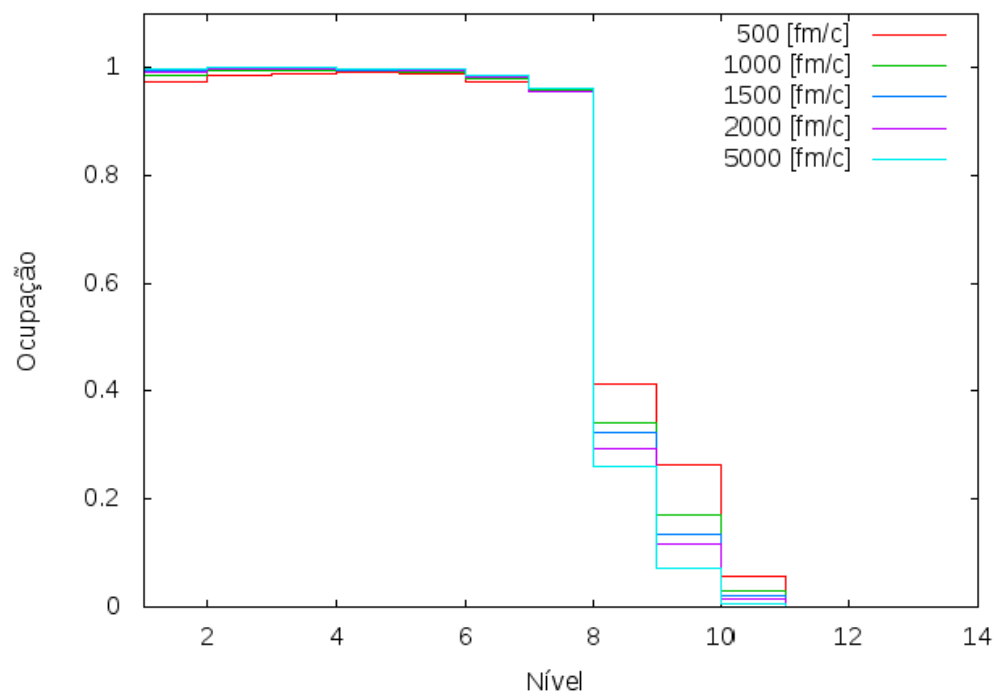

FIGURA 2.6: Distribuição do número de ocupação para nêutrons para vários instantes da cascata. Figura obtida da Ref. [92].

\subsubsection{Ressonâcias nucleônicas}

No código foram feitas melhorias para tornar o processo de simulação das reações nucleares mais realista. Foram incluídas as duas ressonâncias nucleônicas mais importantes após a Delta: as $D_{13}$ e $F_{35}$. A formação e a desintegração das ressonâncias nucleônicas são os mecanismos de maior importância em reações nucleares, principalmente nas reações fotonucleares. Essas alterações foram realizadas para melhorar o cálculo das fotorreações no intervalo de energia entre 500 e $1000 \mathrm{MeV}$. Decaimentos possíveis com as suas respectivas amplitudes são:

$$
\begin{aligned}
& N^{*}\left(P_{33}\right) \rightarrow n+\pi(100 \%) \\
& N^{*}\left(D_{13}\right) \rightarrow n+\pi(50 \%) \\
& N^{*}\left(D_{13}\right) \rightarrow n+\Delta+\pi \rightarrow n+\pi+\pi(25 \%) \\
& N^{*}\left(D_{13}\right) \rightarrow n+\rho \rightarrow n+\pi+\pi(25 \%) \\
& N^{*}\left(F_{35}\right) \rightarrow n+\pi(15 \%) \\
& N^{*}\left(F_{35}\right) \rightarrow \Delta+\pi \rightarrow n+\pi(25 \%) \\
& N^{*}\left(F_{35}\right) \rightarrow n+\rho \rightarrow n+\pi+\pi(60 \%)
\end{aligned}
$$


Chapter 2. O modelo de cascata intranuclear e Evaporação/Fissão usando o método de

O decaimento destas ressonâncias é descrito pela equação de Breit-Wigner

$$
\sigma=\sigma_{0} \frac{\left(\frac{\Gamma}{2}\right)^{2}}{\left(E-E_{0}\right)+\left(\frac{\Gamma}{2}\right)^{2}}
$$

onde $E_{0}$ é a energia da ressonância, $\Gamma$ é o comprimento do canal e $\sigma_{0}$ é o respectivo valor máximo da seção de choque.

\subsubsection{A dinâmica da cascata intranuclear}

No código CRISP, o mecanismo de interação inicial inicia o processo de cascata intranuclear pela modificação do momento de nucleons e/ou a criação de novas partículas, configurando assim o estado exitado inicial do núcleo. As partículas do núcleo excitado evoluem pelo algoritmo da dinâmica da cascata intranuclear cujos aspectos básicos serão abordados nesta seção. O processo de dinâmica da cascata intranuclear inclui a evolução temporal de colisões binárias múltiplas e emissões de pré-equilíbrio, até que o sistema evolua para um estado de equilíbrio termodinâmico. Outras questões abordadas são os critérios para as interações binárias, o balanço energético nas emissões de pré-equilíbrio do processo e o critério de parada da cascata.

Uma das características do código CRISP é a independência do algoritmo da dinâmica da cascata intranuclear do mecanismo de interação inicial da reação. Com isso o CRISP pode ser usado para a simulação de muitos processos nucleares simplesmente pela adaptação de novo mecanismo de interação inicial, provendo assim uma grande liberdade de sistemas a serem estudados.

\subsubsection{A evolução temporal da cascata}

Seja $t_{0}$ o instante de tempo onde a configuração da excitação nuclear foi estabelecida e o instante de tempo de ocorrência $t_{c}$ (expresso em unidades $\mathrm{fm} / \mathrm{c}$ ) para todas as possíveis colisões de pares de partículas ligadas $i-j$. Se cumpre que:

$$
\begin{aligned}
& t_{c}-t_{0}=\frac{r_{i j} \cos \theta}{v_{i j}}, \quad \text { para } \quad \theta<\frac{\pi}{2} \\
& t_{c}-t_{0} \rightarrow \infty \quad \text { para } \quad \theta \geq \frac{\pi}{2}
\end{aligned}
$$

Onde $r_{i j}=\left|\boldsymbol{r}_{i j}\right|=\left|\boldsymbol{r}_{i}-\boldsymbol{r}_{j}\right|$ e $v_{i j}=\left|\boldsymbol{v}_{i j}\right|=\left|\boldsymbol{v}_{i}-\boldsymbol{r}_{j}\right|$ as distâncias e velocidades relativas, respectivamente das partículas envolvidas numa colisão binária e $\cos \theta=\frac{\boldsymbol{r}_{i j} \boldsymbol{v}_{i j}}{r_{i j} v_{i j}}$. Em seguida, usando um tratamento semiclássico pode-se estabelecer se haverá uma interação entre as partículas $i$ e $j$. 
Chapter 2. O modelo de cascata intranuclear e Evaporação/Fissão usando o método de

A condição para que os pares de partículas interajam é:

$$
\pi b_{i j}^{2} \leq \sigma\left(s_{i j}\right)
$$

onde $b_{i j}$ é o parâmetro de impacto e $s_{i j}$ é a seção de choque total de espalhamento em função da energia no centro de massa do sistema binário

$$
\sqrt{s_{i j}}=\sqrt{\left(E_{i}+E_{j}\right)^{2}-\left(\boldsymbol{p}_{i}+\boldsymbol{p}_{j}\right)^{2}} \text {. }
$$

No processo são calculados os intervalos de tempo $\left(t_{c}-t_{0}\right)$ para todos os pares de partículas $i, j$ e, em seguida é escolhido o menor deles $\left(t_{c}-t_{0}\right)_{\min }$ correspondente ao par proposto que produzirá a próxima interação se a condição 2.29 se cumprir. Neste caso é calculada a energia das partículas de acordo com as leis de conservação do momento e energia usando um ângulo obtido da seção de choque diferencial da colisão. Finalmente os momentos das partículas do estado final da colisão são usados para o teste final: o mecanismo de bloqueio de Pauli. No caso de uma interação não bloqueada, todas as variaveís cinemáticas como são as posições e os momentos $p_{\nu}$ de todas as partículas dentro do núcleo são atualizadas. Se a interação é bloqueada o processo se repete para a colisão correspondente ao próximo intervalo de tempo mínimo.

Os processos físicos envolvidos na cascata intranuclear do código CRISP são: i) espalhamento elástico entre nucleons; ii) produção e desintegração de ressonâncias; e iii) espalhamento elástico e inelástico méson - nucleon, para o caso dos píons é incluída a maioria dos processos fundamentais para energias $E_{\pi} \leq 6.0 \mathrm{GeV}$. As seções de choque das colisões por dispersão inelástica, o processo básico de dispersão de energias médias onde ainda não é suficiente para a formação de partículas secundárias, são parametrizadas de acordo às seções de choque de Kikuchi-Kawai [93] na forma:

$$
\begin{array}{r}
\sigma_{n-n, n-p}(\beta)=\left(\frac{10.63}{\beta^{2}}-\frac{29.92}{\beta}+42.9\right) \\
\sigma_{n-p}(\beta)=\left(\frac{34.10}{\beta^{2}}-\frac{82.2}{\beta}+82.2\right)
\end{array}
$$

onde $\beta$ é a velocidade da partícula incidente. Essa parametrização corresponde à descrição de experimentos feitos na faixa de energia de 25 aos $300 \mathrm{MeV}$ com erro de $6 \%$.

\subsubsection{Emissões de pré-equilíbrio e balanço energético}

Na fase dinâmica da cascata intranuclear, além dos processos de espalhamento binários e decaimentos de partículas, acontecem ao mesmo tempo processos de transmissão e 
Chapter 2. O modelo de cascata intranuclear e Evaporação/Fissão usando o método de

de reflexão de nucleons na superfície nuclear. Estes processos são incluídos de forma consistente no código CRISP, uma vez que é assumida a altura do potencial nuclear como a soma da energia de Fermi e da energia de ligação; não existe qualquer restrição especial para simular os processos anteriormente ditos. Estas tem lugar uma vez que as partículas podem obter quantidades suficientes de energia para superar a barreira de potencial da forma:

$$
\begin{array}{r}
T_{\text {cin }}^{0}>V_{0} \text { para partículas neutras } \\
T_{\text {cin }}^{0}>V_{0}+V_{C} \quad \text { para partículas carregadas }
\end{array}
$$

Também está incluído na simulação da cascata, o efeito túnel através da barreira de Coulomb usando a probabilidade calculada anteriormente em (2.19), para que as partículas carregadas tenham uma probabilidade real de serem emitidas mesmo quando a emissão é energeticamente impossível $\left(T_{\text {cin }}^{0}<V_{0}+V_{C}\right)$.

Durante as emissões de pré-equilíbrio, é assumido que a partícula emitida não interaja mais com o núcleo e a energia de recuo do núcleo é insignificante. Este pressuposto simplifica substancialmente os cálculos sem a introdução de erros significativos $\sim \frac{1}{A_{\text {res }}}$ no momento da partícula emitida. O erro é calculado usando a conservação do momento na emissão da partícula $j$, que é expresso por:

$$
\sum_{i=1}^{A} \boldsymbol{P}_{\boldsymbol{i}}=\sum_{i=1(i \neq j)}^{A} \boldsymbol{P}_{\boldsymbol{i}}^{\prime}+\boldsymbol{P}_{j}^{\prime}
$$

onde a soma é sobre todos os nucleons ligados e $\boldsymbol{P}_{\boldsymbol{i}}^{\prime}$ se refere ao momento da partícula $i$ após a emissão. A conservação da energia do sistema para os momentos antes e depois da emissão da partícula é da forma:

$$
\sum_{i=1(i \neq j)}^{A}\left[\left(p_{i}^{2}+\langle m\rangle^{2}\right)^{\frac{1}{2}}-\left(p_{i}^{2}+\left\langle m^{\prime}\right\rangle^{2}\right)^{\frac{1}{2}}\right]=\left(p_{j}^{2}+m_{0}^{2}\right)^{\frac{1}{2}}-\left(p_{j}^{2}+\langle m\rangle^{2}\right)^{\frac{1}{2}}
$$

onde $\langle m\rangle$ é a massa do núcleo. Todos os termos da equação são conhecidos exceto $\left\langle m^{\prime}\right\rangle$. Neste caso assume-se que os $\left\langle m^{\prime}\right\rangle$ são proporcionais ao $\langle m\rangle$, da forma:

$$
\left\langle m^{\prime}\right\rangle=(1-\delta)\langle m\rangle
$$

Os núcleos pesados satisfazem $\delta \ll 1$. Em seguida, expandindo o termo energia que contém a massa desconhecida, em uma série de Taylor na base de $\delta$ e escolhendo o 
Chapter 2. O modelo de cascata intranuclear e Evaporação/Fissão usando o método de

termo de primeira ordem é obtido:

$$
\begin{aligned}
\left(p_{i}^{2}+\left\langle m^{\prime}\right\rangle^{2}\right)^{\frac{1}{2}} & =\left[p_{i}^{2}+(1-\delta)^{2}\left\langle m^{*}\right\rangle^{2}\right]^{\frac{1}{2}} \approx\left(p_{i}^{2}+\langle m\rangle^{2}-2 \delta\langle m\rangle^{2}\right)^{\frac{1}{2}} \\
& \approx\left(1-\frac{\delta\langle m\rangle^{2}}{p_{i}^{2}+\langle m\rangle^{2}}\right)\left(p_{i}^{2}+\langle m\rangle^{2}\right)^{\frac{1}{2}}
\end{aligned}
$$

se (2.38) é substituído em (2.36) é obtido:

$$
\delta\langle m\rangle^{2} \sum_{i=1(i \neq j)}^{A}\left(p_{i}^{2}+\langle m\rangle^{2}\right)^{-\frac{1}{2}}=\left(p_{j}^{2}+m_{0}^{2}\right) \frac{1}{2}-\left(p_{j}^{2}+\langle m\rangle^{2}\right) \frac{1}{2}
$$

e assim:

$$
\delta=\frac{\left(p_{j}^{2}+m_{0}^{2}\right) \frac{1}{2}-\left(p_{j}^{2}+\langle m\rangle^{2}\right) \frac{1}{2}}{\langle m\rangle^{2} \sum_{i=1(i \neq j)}^{A}\left(p_{i}^{2}+\langle m\rangle^{2}\right)^{-\frac{1}{2}}}
$$

Assim a diferença de energia introduzida devido à aproximação é:

$$
\Delta E_{N}=\sum_{i=1(i \neq j)}^{A}\left[\left(p_{i}^{2}+\langle m\rangle^{2}\right)^{\frac{1}{2}}-\left(p_{i}^{2}+\left\langle m^{\prime}\right\rangle^{2}\right)^{\frac{1}{2}}\right]-\left[\left(p_{j}^{2}+m_{0}^{2}\right)^{\frac{1}{2}}-\left(p_{j}^{2}+\langle m\rangle^{2}\right)^{\frac{1}{2}}\right]
$$

É importante assinalar que o processo inicial de excitação nuclear não tem efeito sobre o balanço energético. Esta abordagem tem influência sobre o cálculo do valor da energia liberada na reação porque é introduzida uma incerteza no valor final. Por exemplo para uma cascata $\gamma(140 \mathrm{MeV})$ de ${ }^{208} \mathrm{~Pb}$, é introduzida uma incerteza de $\Delta E \sim 0.27 \mathrm{KeV}$ por cada partícula emitida.

\subsubsection{O critério energético de parada e o cálculo da energia de excitação}

Uma das características dos modelos INC é o critério de parada da cascata intranuclear. Modelos anteriores usaram parâmetros dependentes da energia inicial para resolver esse problema [40, 40, 48, 94-97], que são obtidos através da análise de alguns observáveis, como por exemplo a energia de excitação. Este procedimento é uma limitação para a descrição temporal adequada das fases da cascata de acordo com o trabalho feito pelo grupo de Liège onde demostra que há diferentes tempo de parada para diferentes observáveis de referência [41]. Uma das características do modelo CRISP é o tempo de parada da cascata intranuclear baseado num critério totalmente energético livre de parâmetros.

A fase rápida do mecanismo da cascata intranuclear continua evoluindo enquanto exista pelo menos uma partícula que tem energia suficiente para sair do núcleo. No momento em que todos os nucleons tiverem uma energia cinética menor do que o potencial nuclear 
Chapter 2. O modelo de cascata intranuclear e Evaporação/Fissão usando o método de

do sistema a fase rápida da cascata intranuclear encerra deixando o núcleo num estado estacionário. Este núcleo residual em estado estacionário decai na fase de competição evaporação/fissão. Este tipo de abordagem é mais realista desde que cada cascata tem o seu próprio tempo de parada, mesmo sendo inicializada pelo mesmo mecanismos de interação a mesma energia.

Para energias acima do limiar dos píons é necessária a adição de critérios de paradas adicionais porque nesta faixa de energia podem existir mésons e ressonâncias no meio nuclear. Neste regime de energia é necessário acrescentar ao critério de parada existente a exigência que todas as ressonâncias deveram decair e os mésons deveram estar fora do meio nuclear. Só assim o processo de cascata intranuclear é interrompido.

A energia de excitação da partícula emitida depois é:

$$
E^{*}=E^{*^{\prime}}-\sum_{j}\left(T_{j}^{0}+B\right)
$$

onde $E^{*}$ e $E^{*^{\prime}}$ são as energias de excitação depois e antes da emissão das $j$ partículas respectivamente $T_{j}^{0}$ é a energia cinética assintótica da $j$-ésima partícula para $r \rightarrow \infty$ e $B$ é a energia de ligação média dos nucleons de valência. Na figura 2.7 é mostrado um processo de emissão no qual a energia é transferida por um fóton a um nucleon que escapa do núcleo, cuja energia de excitação diminui no valor da energia cinética da partícula emitida e a energia de ligação.

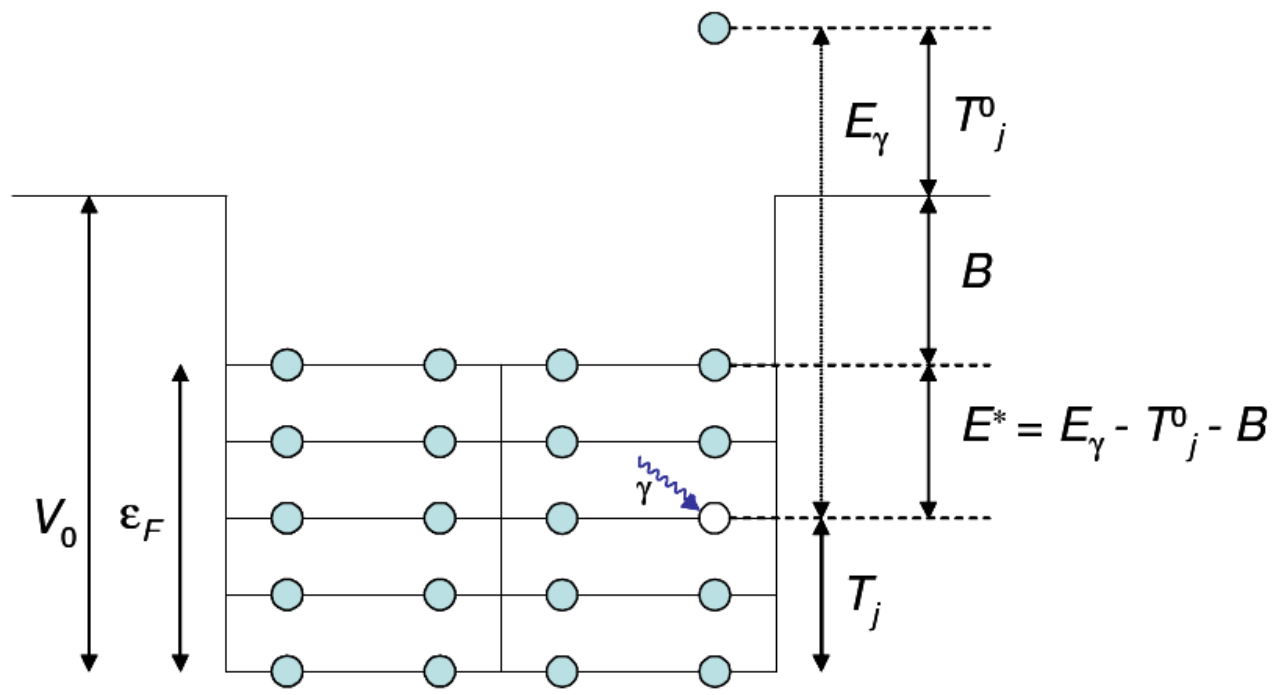

Figura 2.7: Representação de um processo fictício em que um fóton transfere toda sua energia a um nucleon e escapa do núcleo sem interagir com os outros nucleons 
Chapter 2. O modelo de cascata intranuclear e Evaporação/Fissão usando o método de

\subsubsection{Efeito de sombreamento}

Para fótons com energia acima de $\sim 1 G e V$ a função de onda deixa de ser puramente eletromagnética, passando a ter uma componente hadrônica que se manifesta através de auto-estados correspondentes a mésons vetoriais $(\rho, \omega, \phi, \mathrm{J} / \Psi$, etc.) $[98-100]$. O estado hadrônico é caracterizado por uma energia $E_{h}$, módulo do quadrimomento $p$ e massa $m$ relacionados pela lei de conservação [101]:

$$
E_{h}=\left[p^{2} c^{2}+m^{2} c^{4}\right]^{1 / 2} .
$$

A energia $E_{\gamma}$ é a energia do fóton e $\mu=m^{2} c^{4}$. A relação

$$
E_{h}=E_{\gamma}=\left[p^{2} c^{2}+\mu\right]^{1 / 2} \Rightarrow p=\frac{\sqrt{E_{\gamma}^{2}-\mu}}{c}
$$

leva a uma variação da quantidade de movimento dada por:

$$
\Delta p=\frac{E_{\gamma}}{c}-p=\frac{E_{\gamma}}{c}\left[1-\sqrt{1-\frac{\mu}{E_{\gamma}^{2}}}\right] .
$$

Expandindo a raiz quadrada em série de Taylor obtemos:

$$
\Delta p=\frac{1}{2} \frac{\mu}{c E_{\gamma}}
$$

Usando o princípio da incerteza, concluímos que o fóton pode ocupar o seu estado hadrônico por uma distância

$$
\Delta l=\frac{2 \hbar c E_{\gamma}}{\mu^{2}}
$$

Esta expressão mostra que quando a energia do fóton é suficientemente alta a distância percorrida pelo estado hadrônico pode ser grande suficiente para que o méson interaja com os nucleons do meio. A seção de choque de interação com o nucleon é muito diferente para cada um desses estados. De fato, a parte hadrônica da função de onda do fóton interage mais forte, tendo uma seção de choque de ordens de grandeza superior à parte eletromagnética, o que tem consequências na seção de choque de fotoabsorção total do nucleon, que passa a ter um comportamento semelhante àquele para a interação com hádrons. Este efeito pode-se ver nos diagramas da figura 2.8 e 2.9 representado pela "sombra" detrais do nucleon cujo comprimento está dado pela energia $E_{\text {gamma }}$ e a seção de choque de interação (área verde). Na primeira figura é mostrado um efeito de sombreamento ainda não saturado que varia com a energia de acordo a equação 2.47 entanto na segunda figura é mostrado um efeito de sombreamento saturado para energias suficientemente altas. 
Chapter 2. O modelo de cascata intranuclear e Evaporação/Fissão usando o método de

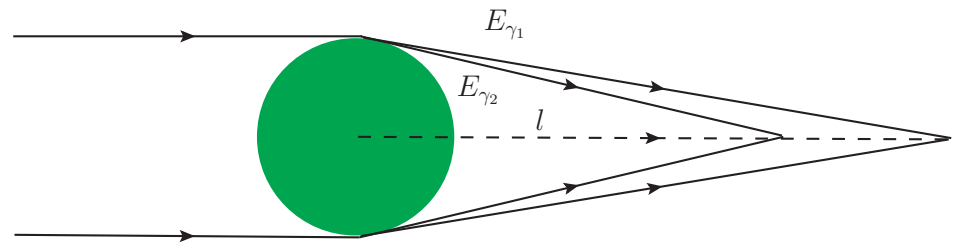

FiguRA 2.8: Diagrama do efeito de sombreamento não saturado do fóton no núcleo.

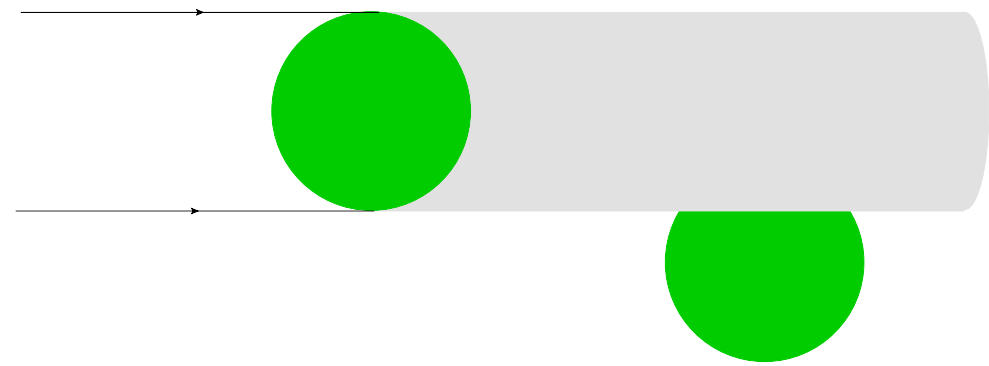

Figura 2.9: Diagrama do efeito de sombreamento saturado do fóton no núcleo.

Com o CRISP foram eliminados alguns efeitos indesejáveis dos softwares mais antigos que não são fisicamente consistentes com a realidade. Ao contabilizar o efeito de sombreamento nos cálculos observamos que em energias intermediárias (até $\sim 2.5 \mathrm{GeV}$ ) o CRISP ajusta muito bem os dados experimentais [17], o que indica que o modelo adotado no software descreve bem as reações nucleares como é mostrado na figura 2.10.

\subsection{Modelo de Evaporação/ Fissão}

Embora no trabalho apresentado aqui não tenha sido utilizada a parte de evaporação/fissão do código CRISP, para fins de complementação descrevemos sucintamente como esta fase da reação nuclear é considerada nosso código.

Depois que ocorre a cascata intranuclear, um núcleo residual em equilibrio termodinâmico é formado e a energia de excitação é distribuída uniformemente entre todos os nucleons. O mecanismo de desexcitação do núcleo se dá pela competição entre os processos de evaporação e fissão nuclear, no qual alguns nucleons conseguem escapar do ambiente nuclear ou o núcleo fissiona formando dois núcleos menores. Todo o processo é determinado pela probabilidade de cada evento calculada por modelos teóricos. Nesta seção serão descritos os métodos de cálculos utilizados no processo de competição entre evaporação/fissão do núcleo composto. 
Chapter 2. O modelo de cascata intranuclear e Evaporação/Fissão usando o método de

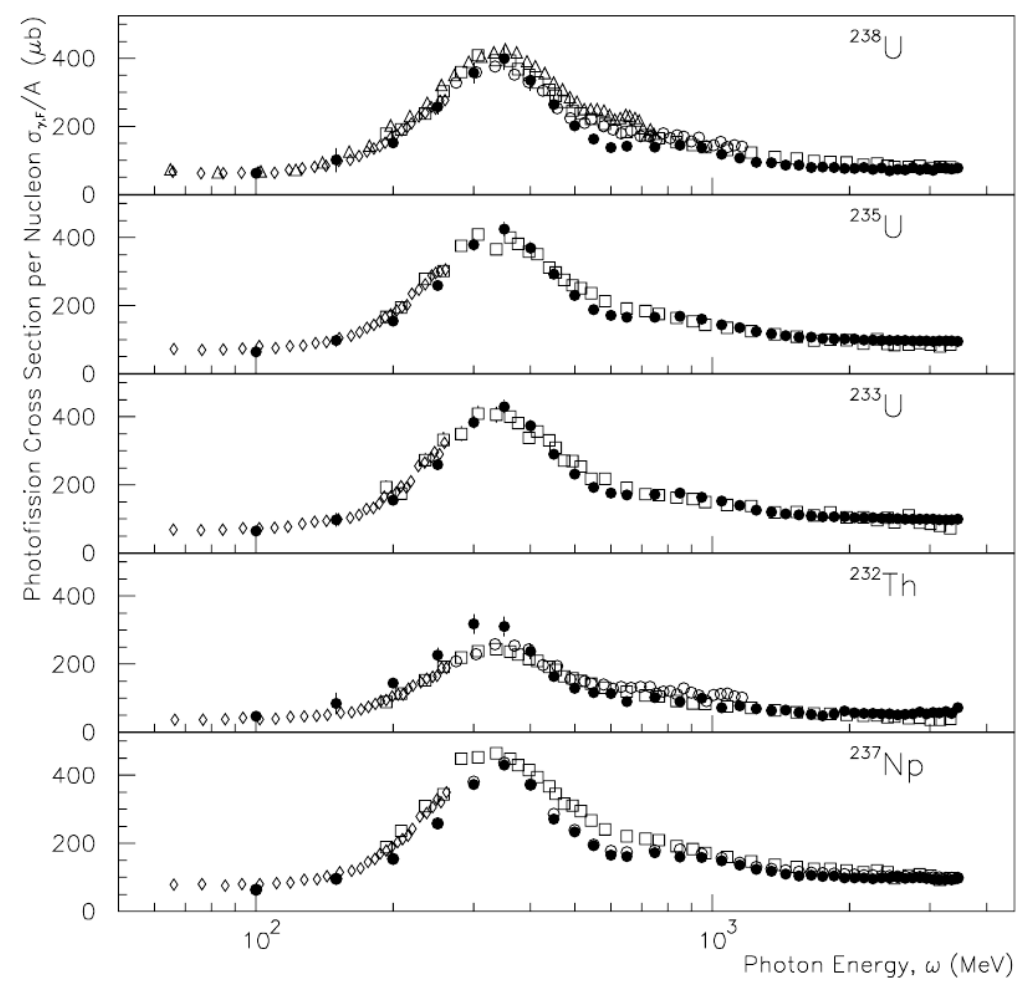

Figura 2.10: Sessão de choque da fotofissão na região de ressonância nucleônica obtida com o código CRISP comparada com dados experimentais. Figura publicada na Ref. [17].

\subsubsection{Descrição do Modelo de Evaporação/ Fissão}

O processo de evaporação nuclear após a formação do núcleo composto é descrito por algoritmo baseado no método de Monte Carlo que calcula em cada etapa da cadeia de evaporação as probabilidades das emissões de partículas e de fissão nuclear. O modelo é baseado no código MCEF[53]. No processo de evaporação só é levada em consideração a emissão de nêutrons, prótons e partículas alfa. Novas correções também estão incluídas no modelo de camadas para calcular a massa nuclear [102]. A figura 2.11 mostra o diagrama do processo de evaporação/fissão que está incluído no CRISP.

Durante a evaporação nuclear a probabilidade de emissão da partícula $k$ sobre a probabilidade de emissão da partícula $j$ é dada pelo modelo estatístico de Weisskopf [103]:

$$
\frac{\Gamma_{k}}{\Gamma_{j}}=\left(\frac{\gamma_{k}}{\gamma_{j}}\right)\left(\frac{E_{k}^{*}}{E_{j}^{*}}\right)\left(\frac{a_{j}}{a_{k}}\right) \exp \left\{2\left[\left(a_{k} E_{k}^{*}\right)^{\frac{1}{2}}-\left(a_{j} E_{j}^{*}\right)^{\frac{1}{2}}\right]\right\}
$$




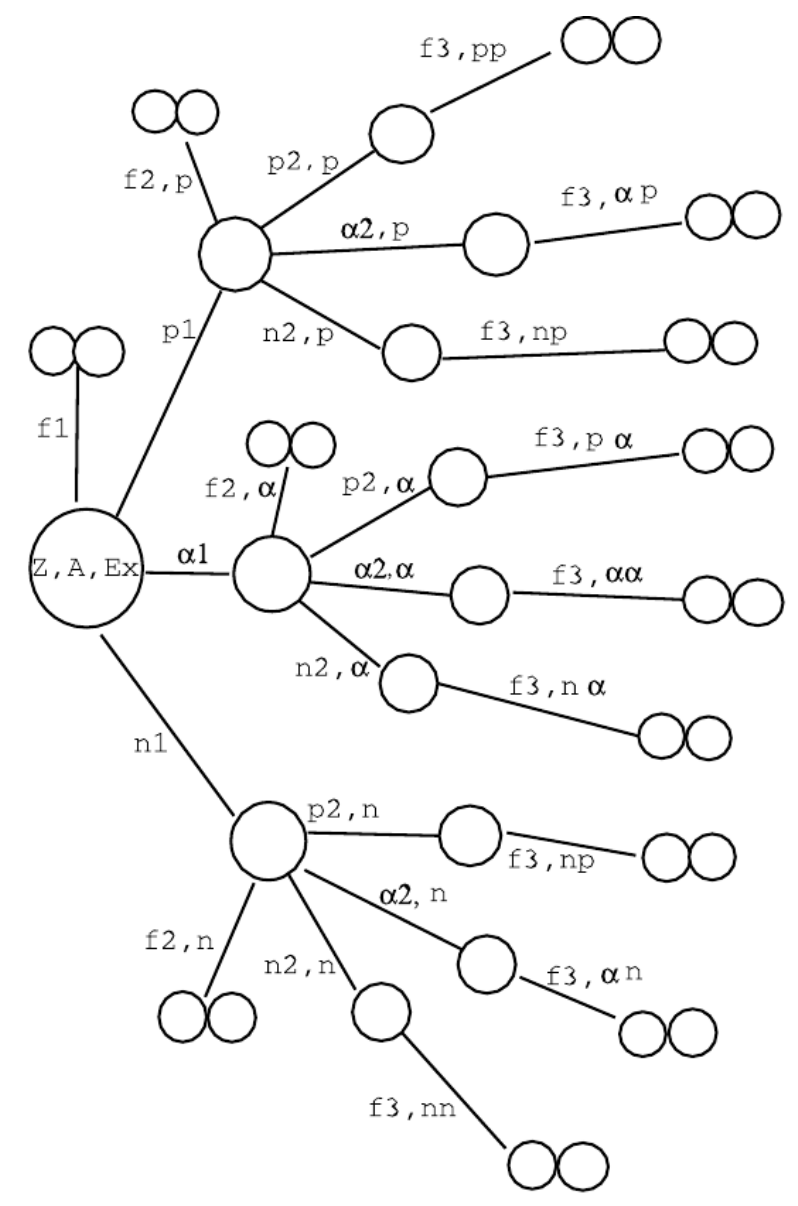

Figura 2.11: Diagrama do processo de competição evaporação/fissão que acontece no núcleo residual simulado no CRISP. Figura publicada na Ref. [92].

para a emissão de nêutrons, o parâmetros das densidades de níveis estão dadas pelas fórmulas empíricas de Dostrovsky [104]:

$$
\begin{gathered}
a_{n}=\frac{A}{a_{1}}\left(1-a_{2} \frac{A-2 Z}{A^{2}}\right)^{2} \\
a_{p}=\frac{A}{a_{3}}\left(1+a_{4} \frac{A-2 Z}{A^{2}}\right)^{2} \\
a_{n}=\frac{A}{a_{5}}\left(1-\frac{a_{6}}{A}\right)^{2}
\end{gathered}
$$

onde $a_{i}$ são parâmetros livres. A razão de probabilidade de emissão entre duas partículas emitidas é dada usualmente relativa aos nêutrons. Para o caso dos prótons a razão é:

$$
\frac{\Gamma_{p}}{\Gamma_{n}}=\left(\frac{E_{p}^{*}}{E_{n}^{*}}\right) \exp \left\{2\left(a_{n}\right)^{\frac{1}{2}}\left[\left(r_{p} E_{p}^{*}\right)^{\frac{1}{2}}-\left(E_{n}^{*}\right)^{\frac{1}{2}}\right],\right\}
$$

e para as partículas alfa:

$$
\frac{\Gamma_{\alpha}}{\Gamma_{n}}=\left(\frac{2 E_{\alpha}^{*}}{E_{n}^{*}}\right) \exp \left\{2\left(a_{n}\right)^{\frac{1}{2}}\left[\left(r_{\alpha} E_{\alpha}^{*}\right)^{\frac{1}{2}}-\left(E_{n}^{*}\right)^{\frac{1}{2}}\right] \cdot\right\}
$$


Chapter 2. O modelo de cascata intranuclear e Evaporação/Fissão usando o método de

O potencial coulombiano para o próton é dado pela fórmula:

$$
V_{p}=C \frac{\left[K_{p}(Z-1) e^{2}\right]}{r_{0}(A-1)^{\frac{1}{3}}+R_{p}},
$$

e para a partícula alfa é:

$$
V_{\alpha}=C \frac{\left[2 K_{\alpha}(Z-2) e^{2}\right]}{r_{0}(A-4)^{\frac{1}{3}}+R_{\alpha}},
$$

onde os coeficientes de penetrabilidade da barreira de Coulomb para prótons e partículas alfa são $K_{p}=0.70$ e $K_{\alpha}=0.83$, respectivamente. Os raios são $R_{p}=1.14 \mathrm{fm}$ e $R_{\alpha}=$ $2.16 \mathrm{fm}, r_{0}=1.2 \mathrm{fm}$ e $C$ é uma constante relacionada à correção do potencial devido à temperatura nuclear. Além disso é usado $r_{p}=r_{\alpha}=1$.

Para o cálculo da probabilidade da fissão nuclear é usado o modelo da gota líquida do modelo de Bohr e Wheeler[47]. A taxa de probabilidade de fissão em relação à probabilidade de emissão de nêutrons é:

$$
\frac{\Gamma_{f}}{\Gamma_{n}}=K_{f} \exp \left\{2\left[\left(a_{f} E_{f}^{*}\right)^{\frac{1}{2}}-\left(a_{n} E_{n}^{*}\right)^{\frac{1}{2}}\right]\right\}
$$

onde

$$
K_{f}=K_{0} a_{n} \frac{\left[2\left(a_{f} E_{f}^{*}\right)^{\frac{1}{2}}-1\right]}{\left(4 A^{\frac{2}{3}} a_{f} E_{n}^{*}\right)}
$$

e

$$
E_{f}^{*}=E^{*}-B_{f}
$$

sendo $K_{0}=14.39 \mathrm{MeV}$, e $B_{f}$ a altura da barreira de fissão.

A energia de ligação da partícula alfa e o próton podem ser calculadas diretamente da sua definição:

$$
\begin{array}{r}
B_{p}=m_{p}+m_{A-1, Z-1}-m_{A, Z} \\
B_{\alpha}=m_{\alpha}+m_{A-4, Z-2}-m_{A, Z} .
\end{array}
$$

Para a energia de ligação dos nêutrons e a barreira de fissão é aplicada uma fórmula empírica que resulta em dados mais precisos:

$$
B_{n}=C(0.22(A-Z)-1.40 Z+101.5) M e V
$$

a barreira de fissão é dada pelo modelo de Nix que faz uso do modelo da gota líquida, onde a energia do núcleo que é a energia que a partícula tem que vencer é dada por [105]:

$$
E_{g l}(N, Z, f)=\left[B_{s}(f)-1\right]+2 x_{0}\left[B_{c}(f)-1\right] E_{s}^{0}
$$


Chapter 2. O modelo de cascata intranuclear e Evaporação/Fissão usando o método de

onde $f$ se refere a forma do núcleo, $B_{s}$ é o termo da superfície que é uma integral unidimensional quando se limita o problema à simetria axial. $B_{c}$, para simetria axial, é o termo coulombiano e é uma integral bidimensional que envolve integrais elípticas completas de primeira e segunda ordem, ou ainda uma integral tridimensional de integrando simples. Estes parâmetro são ajustados de acordo aos dados experimentais. Se a forma estiver definida em termos de três superfícies quadráticas de revolução o problema recai em funções transcendentais elementares. A energia de superfície para um núcleo esférico $E_{s}^{0}$ é dada pela parametrização de Nix:

$$
E_{s}^{0}=a_{s}\left(1-\kappa I^{2}\right) A^{2 / 3}
$$

sendo $\kappa$ uma constante que determina o decréscimo na energia de superfície com o aumento do excesso de nêutrons, com $I=(N-Z) / A$.

A partícula que irá evaporar é selecionada aleatoriamente usando os pesos estatísticos de cada canal. Quando uma dessas partículas é escolhida, os números de massa atômica e de carga são recalculados

$$
\begin{aligned}
& A_{i+1}=A_{i}-\Delta A_{i} \\
& Z_{i+1}=Z_{i}-\Delta Z_{i},
\end{aligned}
$$

onde $\Delta A_{i}$ e $\Delta Z_{i}$ são as massas e a carga das partículas emitidas. Da mesma forma a energia de excitação é modificada de acordo a expressão:

$$
E_{i+1}^{*}=E_{i}^{*}-B_{i}-T_{i}
$$

onde $B_{i}$ e $T_{i}$ são as energias de ligação e cinética assintótica para a partícula emitida, respectivamente. Para nêutrons $T=2 \mathrm{MeV}$, para prótons e partículas alfa $T=V_{p}$ e $T=V_{\alpha}$, a equação 2.66 garante que a energia diminua depois de cada passo na evaporação. Este processo continua até que o sistema não tenha energia suficiente para emitir partículas.

Neste processo pode ser calculado o coeficiente de fissibilidade, que é dado por:

$$
W=\sum\left[\prod_{j=0}^{i-1}\left(1-F_{j}\right)\right] F_{i}
$$

onde $F_{j}$ é a probabilidade de fissão no passo $j$ do processo da evaporação é calculado:

$$
F_{i}=\frac{\left(\frac{\Gamma_{f}}{\Gamma_{n}}\right)_{i}}{1+\left(\frac{\Gamma_{f}}{\Gamma_{n}}\right)_{i}+\left(\frac{\Gamma_{p}}{\Gamma_{n}}\right)_{i}+\left(\frac{\Gamma_{\alpha}}{\Gamma_{n}}\right)_{i}}
$$




\section{Capítulo 3}

\section{Colisões de partículas a altas energias}

Neste capítulo se descreve brevemente a teoria do espalhamento de partículas em altas energias. A física das interações de partículas em altas energias ocupa um lugar muito importante desde que os aceleradores de partículas de altas energias e.g. HERA, RHIC, CERN, etc. tornaram-se o principal laboratório de produção de dados experimentais para o entendimento da estrutura das partículas fundamentais e dos campos. Existe um grande interesse nesta área devido aos novos experimentos que estão surgindo com o aumento da energia e que consequentemente explorarão processos não observados atualmente. O objetivo deste capítulo é fazer um sumário das principais características dos processos que acontecem em altas energias e fundamentalmente dos modelos teóricos usados nas colisões introduzidas no código CRISP. Esta revisão bibliográfica foi extraída da Ref. [106]. Também discutiremos a física das interações com grande parâmetro de impacto: as colisões ultraperiféricas (UPCs). Esta é uma aplicação à extensão do código CRISP desenvolvida neste trabalho e é de grande interesse para a comunidade científica. Muitos experimentos deste fenômeno estão sendo feitos na atualidade no LHC para o estudo de várias temáticas na física de altas energias. Neste capítulo analizaremos as caraterísiticas das UPCs e mostraremos a partir da teoría como será o mecanismo de interação inicial necesario para uma correta simulação das UPCs com o código CRISP.

\subsection{Introdução}

Os processos hadrônicos podem ser classificados de acordo o intervalo de energia e do momento transferido: processos soft e hard ${ }^{1}$.

\footnotetext{
${ }^{1}$ Neste trabalho será usada a notação em inglês
} 
soft. São aqueles processos onde a energia é da ordem do raio do hádron: $\mathrm{R} \sim 1 \mathrm{fm}$ e o momento transferido é geralmente pequeno: $|t| \sim 1 / R^{2} \sim$ (algumas centenas de $\mathrm{MeV})^{2}$. A dependência da seção de choque do processo com o momento transferido é exponencial

$$
d \sigma / d t \sim e^{-R^{2}|t|}
$$

assim, os processos com grande valor do momento transferido são suprimidos. Colisões elásticas hádron-hádron e dissociação difrativa são exemplos deste tipo de processo. A grande escala de comprimento característica destes processos soft indica a natureza não perturbativa deles, assim a cromodinâmica quântica perturbativa não é uma teoria adequada para o seu estudo. A teoria usada para o estudo dos processos soft é a teoria de Regge [107]. É indiscutível o sucesso desta teoria fenomenológica de Regge na descrição numa forma unificada de uma longa lista de reações em ausência de outra alternativa teórica, mesmo pelos menos otimistas. $\mathrm{Na}$ física de altas energias a teoria de Regge é como mencionado por Donnachie and Landshoff [108], "uma das grandes verdades da física das partículas". Segundo Regge, nas altas energias, os processos soft são dominados universalmente pela troca de uma partícula virtual chamada o pomeron.

hard. Estes processos podem acontecer no intervalo de energia da largura do raio dos hádron e nas altas energias. Nas altas energias o valor de momento transferido é grande $\left(|t| \gtrsim 1 \mathrm{GeV}^{2}\right)$ e pode ser tratada pela cromodinâmica quântica perturbativa. A dependência da seção de choque é proporcional à uma potência do módulo do logaritmo da energia. Dois exemplos deste tipo de processo são o espalhamento deep inelastic e produção de jatos para grande valor de momento transferido $t$. Mesmo com grande valor de momento transferido, enquanto parte do processo pode ser descrito pela cromodinâmica quantica perturbativa, o processo contem uma parte não perturbativa que é incluída na função de distribuição de glúons e plasma. O estudo deste tipo de processo é feito usando os teoremas de fatorização [109] que permite separar a parte perturbativa e não perturbativa da seção de choque. A parte não perturbativa é universal e pode ser estudada num processo e usado em outro.

O espalhamento hadrônico pode ser classificado como processo de tipo soft. O termo difrativo foi introduzido na física na década de 50 numa analogia com o conhecido fenômeno óptico que acontece quando um feixe de partículas é espalhado por um objeto ou um orifício com dimensão da mesma ordem que o comprimento de onda do feixe. A principal novidade deste tema nos últimos anos é a descoberta de processos que tem propriedades soft e hard ao mesmo tempo e.g. diffractive deep inelastic scattering (DDIS). 
Os processos de espalhamento hadrônico podem ser definidos como aquelas reações onde não acontece troca de números quânticos (com exceção dos números quânticos do vácuo) entre as partículas que colidem nas altas energias. É um processo que acontece assintoticamente eliminando assim a possibilidade de contaminação com processos não difrativos. O espalhamento hadrônico pode ser divididos em vários casos:

1. espalhamento elástico: Onde as partículas incidentes são as mesmas que as partículas que resultam da colisão:

$$
1+2=1^{\prime}+2^{\prime}
$$

2. difração única (single diffraction): Onde uma das partículas incidentes é espalhada enquanto a outra produz várias partículas ou uma ressonância com os mesmos números quânticos.

$$
1+2=1^{\prime}+X_{2}
$$

3. difração dupla (double diffraction): onde cada uma das partículas incidentes produz várias partículas ou uma ressonância com os mesmos números quânticos.

$$
1+2=X_{1}+X_{2}
$$
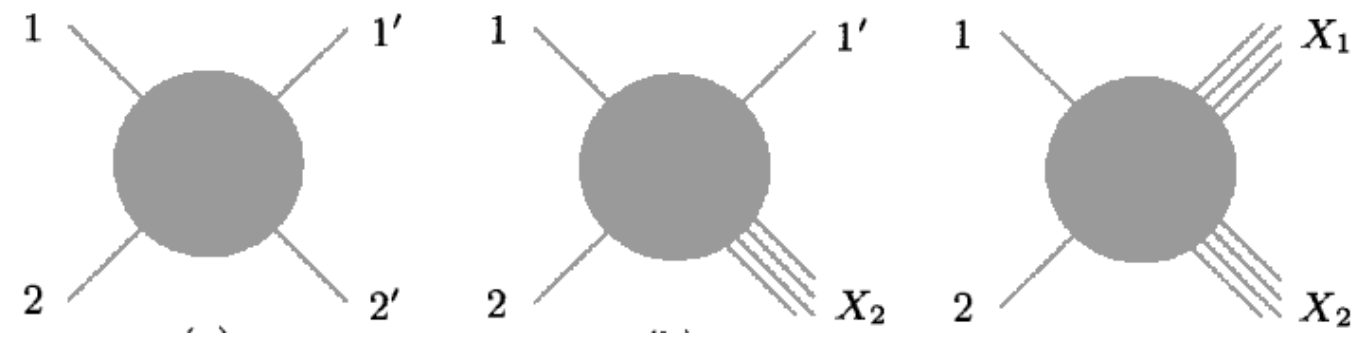

FigurA 3.1: Difração hadrônica. Elástico (esquerda), difração única (centro), difração dupla (direita)

Na pratica é simples identificar uma reação elástica. Não é assim quando não se pode reconstruir totalmente o estado final como acontece no casos 2 e 3 e com isso assegurar a igualdade de números quânticos entre as partículas incidentes e as espalhadas. Devido a issto é conveniente definir o espalhamento hadrônico numa forma mais prática. Para isso é definida uma nova grandeza: a rapidez. Seja uma partícula de energia E e componente do momento $p_{z}$, a rapidez $y$ é definida como:

$$
y=\frac{1}{2} \ln \frac{E+p_{z}}{E-p_{z}}
$$


O que faz da rapidez uma grandeza muito útil é o fato de que ela se transforma aditivamente sob uma transformação de Lorentz no eixo- $z$, i.e:

$$
\begin{gathered}
\left(E, \mathbf{p}_{\perp}, p_{z}\right) \underset{\text { boost }}{\longrightarrow}\left(\gamma\left(E+\beta p_{z}\right), \mathbf{p}_{\perp}, \gamma\left(p_{z}+\beta E\right)\right) \\
y \underset{\text { boost }}{\longrightarrow \longrightarrow} y+\frac{1}{2} \ln \frac{1+\beta}{1-\beta}
\end{gathered}
$$

assim, qualquer diferença de rapidez é invariante sob uma transformação de Lorentz longitudinal. Em geral, a rapidez pode ser expressa dependendo do ângulo de espalhamento $\vartheta$ da forma:

$$
y=\frac{1}{2} \ln \frac{1+\beta \cos \vartheta}{1-\beta \cos \vartheta} .
$$

Para energias ultra relativísticas $\beta \sim 1$ a rapidez é determinada só pelo ângulo de espalhamento. Devido a isso, é definida a pseudo-rapidez:

$$
\eta=\frac{1}{2} \ln \frac{1+\cos \vartheta}{1-\cos \vartheta}=\ln \cot \vartheta / 2 .
$$

Para valores de velocidade próxima à velocidade da luz a pseudo-rapidez e a rapidez são numericamente iguais, exceto nos valores $\vartheta=0, \pi$.

Definindo o gap de rapidez do estado final $\Delta \eta$, o espalhamento hadrônico pode ser também definido como aquelas reações que estão caracterizadas por um elevado valor do gap da rapidez. No estado final, esta grandeza deverá ser suprimida numa forma não exponencial. Um alto valor do gap de rapidez indica um grande ângulo de espalhamento. Como no caso da definição anterior, esta condição é necessária mas não suficiente, desde que podem haver espalhamentos não difrativos. O problema é resolvido desde que nas altas energias os processos não difrativos sejam exponencialmente suprimidos, diferentemente dos difrativos. A distribuição de eventos difrativos é [106]

$$
\frac{d N}{d \Delta \eta} \sim \text { constante }
$$

enquanto a distribuição de eventos não difrativos tem a forma

$$
\frac{d N}{d \Delta \eta} \sim \exp ^{\Delta \eta}
$$

A teoria de Regge é o atual marco teórico para o estudo dos processos difrativos. A teoria descreve os espalhamentos hadrônicos em termos de trocas de "objetos" virtuais chamados de reggeons. O reggeon que contêm números quânticos do vácuo, é chamado de pomeron. Assintoticamente a troca de outros "objetos" com o vácuo, que contribuem ao espalhamento não difrativo é suprimida nas altas energias onde a troca do pomerons domina o processo. 


\subsection{Espalhamento de uma partícula pelo um potencial cen- tral}

Para o estudo de processos difrativos nas altas energias é importante introduzir o espalhamento não-relativístico por um potencial central. Existem duas aproximações: uma tem com base a equação de Schrödinger e outra esta baseada em propriedades gerais da amplitude de espalhamento.

\subsubsection{A aproximação da equação de Schrödinger}

Na mecânica quântica, o movimento relativo de duas partículas sem spin que interagem via o potencial central $V(\mathbf{r})$ esta dado pela conhecida equação de Schrödinger:

$$
-\frac{\hbar^{2}}{2 \mu} \nabla^{2} \psi(\mathbf{r})+V(\mathbf{r}) \psi(\mathbf{r})=E \psi(\mathbf{r})
$$

onde $\mu$ é a massa reduzida do sistema, $V(\mathbf{r})$ é um potencial definido com um alcance de interação finito. A equação pode ser simplificada se:

$$
k^{2}=\frac{2 \mu}{\hbar^{2}} E, \quad U(\mathbf{r})=\frac{2 \mu}{\hbar^{2}} V(\mathbf{r}),
$$

assim a equação reduzida fica:

$$
\left(\nabla^{2}-U(\mathbf{r})-k^{2}\right) \psi(\mathbf{r})=0
$$

$\mathrm{Na}$ teoria do espalhamento de partículas em altas energia, assume-se que a função de onda incidente é uma onda plana enquanto assintoticamente (i.e $r \rightarrow \infty$ ) é uma superposição de uma onda incidente e uma onda esférica que emerge do centro de massa do sistema. Matematicamente isto é:

$$
\psi(\mathbf{r}) \underset{r \rightarrow \infty}{\sim} e^{i \mathbf{k r}}+f\left(\mathbf{k}, \mathbf{k}^{\prime}\right) \frac{e^{i k r}}{r}
$$

onde $\mathbf{k}$ e $\mathbf{k}^{\prime}$ são os vetores de onda da onda incidente e espalhada respectivamente que estão relacionados pela lei de conservação do momento, assim: $|\mathbf{k}|=\left|\mathbf{k}^{\prime}\right| \equiv k$. A grandeza $f\left(\mathbf{k}, \mathbf{k}^{\prime}\right)$ é a amplitude de espalhamento e contém toda a informação do processo de colisão. A solução da equação reduzida de Schrödinger 3.14 que cumpre com a solução assintótica 3.15 é dada pela equação integral de Lippman-Schwinger:

$$
\psi(\mathbf{r})=e^{i \mathbf{k r}}-\frac{1}{4 \pi} \int \frac{e^{i k\left|\mathbf{r}-\mathbf{r}^{\prime}\right|}}{\left|\mathbf{r}-\mathbf{r}^{\prime}\right|} U\left(\mathbf{r}^{\prime}\right) \psi\left(\mathbf{r}^{\prime}\right) .
$$




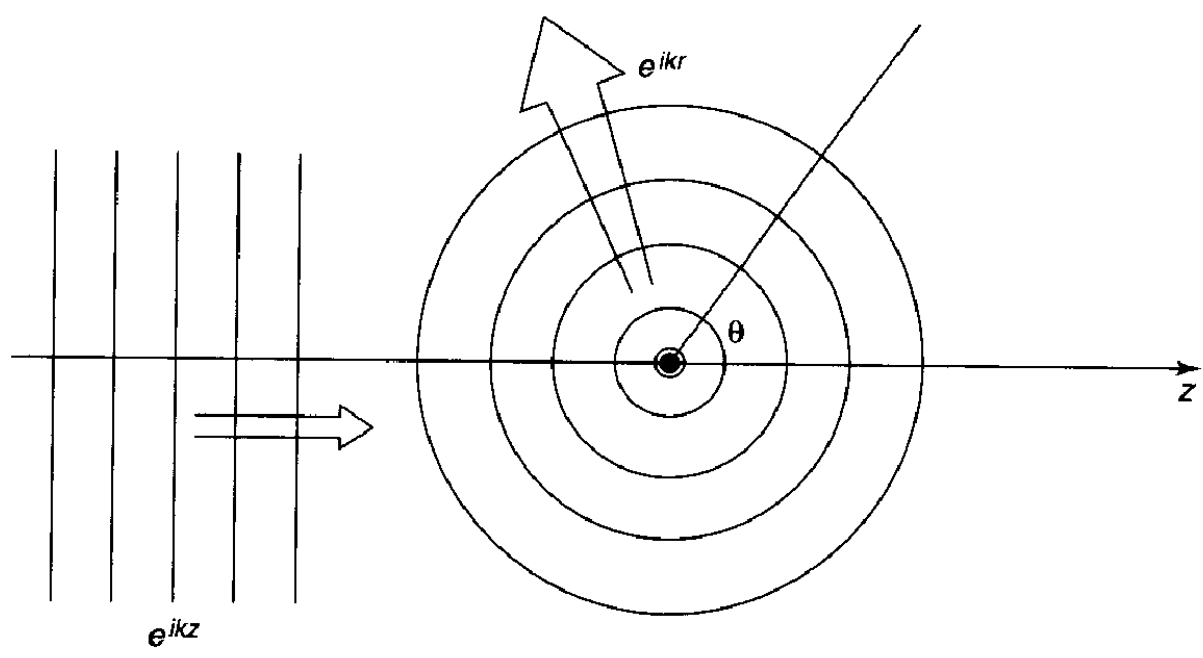

Figura 3.2: Espalhamento de uma partícula por um potencial central [106].

Se o potencial vai a zero mais rapidamente de que $1 / r$ (i.e $r U(r) \underset{r \rightarrow \infty}{\rightarrow} 0$ ), a expressão $\left(\left|\mathbf{r}-\mathbf{r}^{\prime}\right|\right)$ pode ser expandida usando a série de Taylor

$$
\left|\mathbf{r}-\mathbf{r}^{\prime}\right| \simeq r-\mathbf{r}^{\prime} \frac{\mathbf{r}}{r}
$$

assim, comparando as equações 3.16 e 3.15

$$
f\left(\mathbf{k}, \mathbf{k}^{\prime}\right)=-\frac{1}{4 \pi} \int e^{i \mathbf{k}^{\prime} \mathbf{r}} U\left(\mathbf{r}^{\prime}\right) \psi\left(\mathbf{r}^{\prime}\right)
$$

o valor de momento transferido $\mathbf{q}=\mathbf{k}^{\prime}-\mathbf{k}$ pode ser expresso em função do ângulo de espalhamento $\vartheta$ pela relação

$$
q=2 k \sin \frac{\vartheta}{2}
$$

Assim a seção de choque elástica diferencial no ângulo sólido $(d \Omega=d \phi d(\cos \vartheta))$ do processo é

$$
\frac{\sigma_{\text {elas }}}{d \Omega}=|f(k, \vartheta, \phi)|^{2}
$$

A solução para o potencial central é usualmente dada em coordenadas esféricas. Nestas, a amplitude $f$ não depende do ângulo $\phi$ e pode ser expandida em termos de ondas parciais, o que matematicamente se refere à uma expansão em harmônicos esféricos da forma

$$
f(k, \vartheta)=\sum_{l=0}^{\infty}(2 l+1) a_{l}(k) P_{l}(\cos \vartheta),
$$

onde $l$ é o valor do momento angular e $P_{l}$ são os conhecidos polinômios de Legendre que contêm toda a informação angular. A amplitude parcial $a_{l}(l)$ pode ser substituída em 
função da mudança de fase $\delta_{l}$ da onda com momento angular $l[110]$

$$
a_{l}(k)=\frac{e^{2 i \delta_{l}(k)}}{2 i k} \equiv \frac{S_{l}(k)-1}{2 i k}, .
$$

O significado físico da mudança de fase pode ser entendida se for analisado o caráter assintótico das funções de onda radial incidente e espalhada respectivamente, que são funções de Bessel

$$
\begin{aligned}
& u_{l}(k) \underset{r \rightarrow \infty}{\sim} \frac{1}{k r} \sin \left(k r-\frac{\pi l}{2}\right) \\
& \varphi_{l}(k) \underset{r \rightarrow \infty}{\sim} \frac{e^{i \delta_{l}}}{k r} \sin \left(k r-\frac{\pi l}{2}+\delta_{l}\right) .
\end{aligned}
$$

Pode-se notar que a única diferença entre a onda incidente e espalhada é a mudança de fase e é esta que leva toda a informação da dinâmica do processo e toda a informação do potencial.

A seção de choque pode ser integrada pelo ângulo $\phi$, desde que não depende deste

$$
\frac{\sigma_{\text {elas }}}{d \cos \vartheta}=2 \pi|f(k, \vartheta)|^{2}
$$

Substituindo a amplitude radial 3.22 na equação da amplitude de espalhamento $3.21 \mathrm{e}$ usando a condição de ortogonalidade dos polinômios de Legendre, a seção de choque fica:

$$
\begin{aligned}
\sigma_{\text {elas }} & =\int \frac{d \sigma_{\text {elas }}}{d \cos \vartheta} \\
& =\frac{\pi}{k^{2}} \sum_{l=0}^{\infty}(2 l+1)\left|S_{l}-1\right|^{2} \\
& =\frac{\pi}{k^{2}} \sum_{l=0}^{\infty}(2 l+1) \sin ^{2} \delta_{l} .
\end{aligned}
$$

É importante notar que até agora tem considerado unicamente o espalhamento elástico. Para analisar o processo inelástico e com ele os efeitos de absorção no processo, propõese um potencial complexo que produz uma solução complexa que é contida numa nova fase imaginária na solução.

\subsubsection{A aproximação da matriz $\mathrm{S}$}

Nesta seção é introduzida a aproximação da matriz $S$. Esta abordagem está baseada nas propriedades que tem que cumprir a amplitude de espalhamento, assim pode ser usada para estudar os processos inelásticos, partículas com spin, processos de espalhamento 
por um potencial não central, assim como processos dependentes do tempo, reações com vários canais de saída, colisões relativísticas etc.

A matriz de espalhamento $S$ é definida como o operador unitário que transforma o estado inicial $|i\rangle$ do processo de espalhamento no estado final $|f\rangle$, assim

$$
|f\rangle=S|i\rangle
$$

de acordo com esta definição, o operador $S$ está relacionado ao operador unitário de evolução temporal $U\left(t_{1}, t_{2}\right)$ e assim com o hamiltoniano de interação $\mathcal{H}_{\text {int }}^{\prime}$. Usando a representação de interação $S$ pode ser escrita como

$$
S=\mathcal{T} \int_{-\infty}^{\infty} e^{-\frac{i}{\hbar} \mathcal{H}_{\text {int }}^{\prime}(t) d t}
$$

onde $\mathcal{T}$ é o operador time ordering. Esta equação é cohecida como a fórmula de Dayson. O caráter unitário da matriz $S S^{\dagger}=S^{\dagger} S=\hat{\mathbf{1}}$, implica que o estado inicial pode ser obtido da forma $|i\rangle=S^{\dagger}|f\rangle$.

Substituindo explicitamente os estados inicial e final $|i\rangle=|\mathbf{k}, \lambda\rangle$ e $|f\rangle=\left|\mathbf{k}^{\prime}, \lambda^{\prime}\right\rangle$, a seção diferencial do processo pode ser expressa em termo da matriz $S$ :

$$
d \sigma=\frac{4 \pi^{2}}{k^{2}}\left|\left\langle\mathbf{k}^{\prime}, \lambda^{\prime}|S-\hat{\mathbf{1}}| \mathbf{k}, \lambda\right\rangle\right|^{2} d \Omega
$$

O módulo dos vetores do momento inicial e final do sistema de partículas são iguais devido à lei de conservação do momento, assim a diferença entre os estados inicial e final depende unicamente das direções dos vetores $\hat{\mathbf{k}}$ e $\hat{\mathbf{k}}^{\prime}$ ou o que é o mesmo dos ângulos $\vartheta$ e $\phi$. Se os outros números quânticos são denotados pelos índices $\lambda$ e $\lambda^{\prime}$ para os estados inicial e final respectivamente, a normalização dos estados é da forma:

$$
\left\langle\mathbf{k}^{\prime}, \lambda^{\prime} \mid \mathbf{k}, \lambda\right\rangle=\delta_{\lambda \lambda^{\prime}} \delta\left(\hat{\mathbf{k}}-\hat{\mathbf{k}}^{\prime}\right)=\delta_{\lambda \lambda^{\prime}} \delta\left(\Omega_{k}-\Omega_{k^{\prime}}\right)
$$

Até agora as equações descrevem processos de espalhamento gerais, o seja:

$$
\frac{d \sigma}{d \Omega}=\left|f\left(k, \vartheta, \phi ; \lambda, \lambda^{\prime}\right)\right|^{2}
$$

Comparando com a equação 3.31 a amplitude de espalhamento é da forma

$$
f\left(k, \vartheta, \phi ; \lambda, \lambda^{\prime}\right)=\frac{2 \pi i}{k}\left\langle\hat{\mathbf{k}}^{\prime}, \lambda^{\prime}|S-\hat{\mathbf{1}}| \hat{\mathbf{k}}, \lambda\right\rangle .
$$


Expandindo a fórmula de Dayson 3.30 até o primeiro termo, de acordo a aproximação de Born:

$$
S \simeq \hat{\mathbf{1}}-\frac{1}{\hbar} \int_{-\infty}^{\infty} \mathcal{H}_{i n t}^{\prime}(t) d t
$$

para o mesmo nível de energia e usando o princípio de incerteza:

$$
\left\langle\hat{\mathbf{k}}^{\prime}, \lambda^{\prime}|S-\hat{\mathbf{1}}| \hat{\mathbf{k}}, \lambda\right\rangle \simeq-2 \pi i\left\langle\hat{\mathbf{k}}^{\prime}, \lambda^{\prime}\left|\mathcal{H}_{S h c}^{\prime}\right| \hat{\mathbf{k}}, \lambda\right\rangle
$$

onde $\mathcal{H}_{S h c}^{\prime}$ é o hamiltoniano de interação na representação de Schrödinger, assim a amplitude de espalhamento está relacionada com o hamiltoniano $\mathcal{H}_{S h c}^{\prime}$, usando a equação 3.34:

$$
f\left(k, \vartheta, \phi ; \lambda, \lambda^{\prime}\right)=-\frac{4 \pi}{k}\left\langle\hat{\mathbf{k}}^{\prime}, \lambda^{\prime}\left|\mathcal{H}_{S h c}^{\prime}\right| \hat{\mathbf{k}}, \lambda\right\rangle
$$

para o caso de um potencial central os números quânticos são os autovalores do operador momento angular 1 e os autoestados $|l m\rangle$ tem componente no espaço dos momentos da forma:

$$
\langle\hat{\mathbf{k}}, \lambda \mid l m, \lambda\rangle=Y_{l}^{m}(\Omega),
$$

onde $Y_{l}^{m}(\Omega)$ são os harmônicos esféricos. As componentes da matriz $S$ na base $|l m, \lambda\rangle$ cumprem que:

$$
\left\langle l^{\prime} m^{\prime}, \lambda^{\prime}|S| l m, \lambda\right\rangle=\delta_{l l^{\prime}} \delta_{m m^{\prime}} S_{l}^{\lambda \lambda^{\prime}} .
$$

Substituindo esta última relação junto com o teorema de adição dos harmônicos esféricos

$$
\sum_{m=-l}^{l} Y_{l}^{m}\left(\Omega_{k}\right) Y_{l}^{m *}\left(\Omega_{k^{\prime}}\right)=\frac{2 l+1}{4 \pi} P_{l}(\cos \vartheta)
$$

nos termos da matriz $S$ (equação 3.31) resulta:

$$
\left\langle\hat{\mathbf{k}}^{\prime}, \lambda^{\prime}|S-\hat{\mathbf{1}}| \hat{\mathbf{k}}, \lambda\right\rangle=\frac{1}{4 \pi} \sum_{l=0}^{\infty}(2 l+1)\left(S_{l}^{\lambda \lambda^{\prime}}-\delta_{\lambda \lambda^{\prime}}\right) P_{l}(\cos \vartheta)
$$

e a seção de choque:

$$
\frac{d \sigma}{d \Omega}=\frac{1}{4 k^{2}}\left|\sum_{l=0}^{\infty}(2 l+1)\left(S_{l}^{\lambda \lambda^{\prime}}-\delta_{\lambda \lambda^{\prime}}\right) P_{l}(\cos \vartheta)\right|^{2}
$$

assim, usando as propriedades de ortogonalidade dos polinomios de Legendre:

$$
\sigma(k)=\int \frac{d \sigma}{d \Omega} d \Omega=\frac{\pi}{k^{2}} \sum_{l=0}^{\infty}(2 l+1)\left|S_{l}^{\lambda \lambda^{\prime}}-\delta_{\lambda \lambda^{\prime}}\right|^{2} .
$$

Para o caso de espalhamento elástico, os números quânticos $\lambda$ e $\lambda^{\prime}$ são iguais e com eles os únicos termos que são diferentes de zero são os da diagonal $S_{l} \equiv S_{l}^{\lambda \lambda}$. Aplicando o 
anterior a seção de choque é da forma:

$$
\sigma_{\text {elas }}(k)=\frac{\pi}{k^{2}} \sum_{l=0}^{\infty}(2 l+1)\left|S_{l}-1\right|^{2}
$$

e a inelástica:

$$
\sigma_{\text {inel }}(k)=\frac{\pi}{k^{2}} \sum_{l=0}^{\infty}(2 l+1) \sum_{\lambda \neq \lambda^{\prime}}\left|S_{l}^{\lambda \lambda^{\prime}}\right|^{2} .
$$

O caráter unitário da matriz $S$ implica uma relação os termos da diagonal e os não diagonais uma vez que a base $|\lambda l m\rangle$ seja ortogonal, assim:

$$
\sum_{\lambda \lambda^{\prime}}\left|S_{l}^{\lambda \lambda^{\prime}}\right|^{2}=\left|S_{l}\right|^{2}+\sum_{\lambda \neq \lambda^{\prime}}\left|S_{l}^{\lambda \lambda^{\prime}}\right|^{2}=1,
$$

então a seção de choque inelástica pode ser expressa na base dos elementos diagonais da matriz $S$ :

$$
\sigma_{\text {inel }}(k)=\frac{\pi}{k^{2}} \sum_{l=0}^{\infty}(2 l+1)\left(1-\left|S_{l}\right|^{2}\right)
$$

e a seção de choque total $\sigma_{\text {tot }}=\sigma_{\text {elas }}+\sigma_{\text {inel }}$ :

$$
\sigma_{t o t}=\frac{\pi}{k^{2}} \sum_{l=0}^{\infty}(2 l+1)\left(1-\operatorname{Re}\left(S_{l}\right)\right)
$$

Um teorema muito importante resulta da comparação com a equação da expansão em harmônicos esféricos mostradas na eq. 3.21 e usando relação entre os elementos da matriz $S$ a amplitude reduzida (eq. 3.22):

$$
\operatorname{Im} f(0)=\frac{k}{4 \pi} \sigma_{t o t}
$$

Este teorema é conhecido como teorema óptico e é uma consequência direta da unitariedade. Nas colisões reais as seções de choques total e diferencial são obtidas com uma grande precisão. Com estas e usando o teorema óptico é como podem ser determinadas as partes real e imaginária da amplitude de espalhamento.

\subsection{A matriz $\mathrm{S}$ relativística}

A abordagem da matriz $S$ relativística é uma alternativa á teoria quântica de campos para descrever as interações fortes. Mesmo a teoria não ter tido o sucesso esperado nessa área, seus métodos, conceitos e ferramentas ainda são muito usados na física hadrônica, já que são baseados nas características que precisam cumprir os processos de espalhamento em vez do estudo da natureza deste. 
Para altas energias, de acordo a teoria quântica de campos, num processo:

$$
1+2 \rightarrow 3+4+5+6 \ldots+N
$$

a matriz S é dada pela equação:

$$
S=\hat{\mathbf{1}}+\sum_{n=1}^{\infty} \frac{i^{n}}{n !} \int d^{4} x_{1} \ldots d^{4} x_{n} \mathcal{T}\left(\mathcal{H}_{\text {int }}^{\prime}\left(x_{1}\right)\right) \ldots \mathcal{H}_{\text {int }}^{\prime}\left(x_{n}\right)
$$

onde $\mathcal{H}_{\text {int }}^{\prime}$ é o hamiltoniano de interação e $\mathcal{T}$ é o operador time ordering. Ao mesmo tempo pode ser expressa em função do operador ou matriz de transição:

$$
S=\hat{\mathbf{1}}+i T
$$

A teoria relativística da matriz $S$ está baseada propriedades que são usualmente assumidas. Estas são:

- linearidade,

- invariância sob uma transformação de Lorentz

- unitariedade

- analiticidade

- crossing.

A linearidade reflete o princípio de superposição da mecânica quântica, enquanto a propriedade de ser invariante relativística obriga a que os termos da matriz $S$ dependam das variáveis cinemáticas invariantes de Lorentz. A propriedade de unitariedade é uma consequência direta da conservação da probabilidade, enquanto a analiticidade e crossing são basicamente postuladas embora sejam suportadas pela teoria quântica de campos.

Os elementos da matriz relativística $S_{i f}$ são neste caso:

$$
\left.S_{i f} \equiv\right\rangle|S| i\left\langle=\delta_{i j}+i\right\rangle f|T| i\left\langle=\delta_{i j}+(2 \pi)^{4} \delta^{4}\left(p_{i}-p_{f}\right) A(i \rightarrow f)\right.
$$

onde $A(i \rightarrow f)$ é a amplitude relativística do espalhamento. A seção de choque diferencial é:

$$
d \sigma=\frac{1}{\Phi}\left|A\left(i \rightarrow f_{n}\right)\right|^{2} d \Pi_{n} \therefore \sigma_{t o t}=\frac{1}{\Phi} \sum_{n} \int d \Pi_{n}\left|A\left(i \rightarrow f_{n}\right)\right|^{2}
$$


onde $\Phi$ é o fluxo incidente e $d \Pi_{n}$ é o espaço de fase invariante de Lorentz das $n$-partículas. Para duas partículas no estado final:

$$
\frac{d \sigma}{d \Omega}=\frac{\lambda^{1 / 2}\left(s, m_{3}^{2}, m_{4}^{2}\right)}{64 \pi^{2} s \lambda^{1 / 2}\left(s, m_{1}^{2}, m_{2}^{2}\right)}|A(s, t)|^{2}
$$

onde $s$ e $t$ são invariantes de Mandelstam. A altas energias, uma cinemática diferente é usada para descrever a configuração inicial e final do processo. Esta tem que se adaptar à cinemática relativística. Muitas das abordagens teóricas dos processos em altas energias estão baseadas na identificação de regimes cinemáticos para cada tipo de processo. Os processos a altas energias são usualmente descritos usando as variáveis de Mandelstam que são definidas, para este caso:

$$
\begin{aligned}
& s=\left(p_{1}+p_{2}\right)^{2}=\left(p_{3}+p_{4}\right)^{2} \\
& t=\left(p_{1}-p_{3}\right)^{2}=\left(p_{2}-p_{4}\right)^{2} \\
& u=\left(p_{1}-p_{4}\right)^{2}=\left(p_{2}-p_{3}\right)^{2} .
\end{aligned}
$$

As variáveis de Mandelstam cumprem a identidade:

$$
s+t+u=\sum_{i=1}^{4} m_{i}^{2}
$$

A função $\lambda^{1 / 2}(x, y, z)$ é a função triangular definida na forma:

$$
\lambda^{1 / 2}(x, y, z)=x^{2}+y^{2}+z^{2}-2 x y-2 y z-2 x z .
$$

Usando a relação:

$$
d \cos \vartheta=\frac{2 s}{\lambda^{1 / 2}\left(s, m_{1}^{2}, m_{2}^{2}\right) \lambda^{1 / 2}\left(s, m_{3}^{2}, m_{4}^{2}\right)} d t \underset{s \rightarrow \infty}{\simeq} \frac{2}{s} d t
$$

é calculada a seção de choque diferencial em função do momento transferido que é uma grandeza muito reportada na literatura e é expressa na forma:

$$
\frac{d \sigma}{d t}=\frac{1}{16 \pi \lambda^{1 / 2}\left(s, m_{1}^{2}, m_{2}^{2}\right)}|A(s, t)|^{2} \underset{s \rightarrow \infty}{\simeq} \frac{1}{16 \pi s^{2}}|A(s, t)|^{2} .
$$

As propriedades da matriz $S$ levam a que a amplitude de espalhamento satisfaça características muito importantes: 


\subsubsection{Unitariedade}

Uma vez que a condição de unitariedade impõe que:

$$
\left(\hat{\mathbf{1}}-i T^{\dagger}\right)\left(\hat{\mathbf{1}}+i T^{\dagger}\right)=\hat{\mathbf{1}} \therefore i\left(T^{\dagger}-T\right)=T^{\dagger} T,
$$

os elementos matriciais

$$
i\left\langle f\left|T^{\dagger}-T\right| i\right\rangle=\sum_{\{n\}}\left\langle f\left|T^{\dagger}\right| n\right\rangle\langle n|T| i\rangle
$$

e as amplitudes de espalhamento ficam na forma:

$$
2 \operatorname{Im} A(i \rightarrow f)=\sum_{n} \int d \Pi_{n} A^{*}(f \rightarrow n) A(i \rightarrow n) .
$$

Um resultado muito importante sai da relação de unitariedade comparando a equação

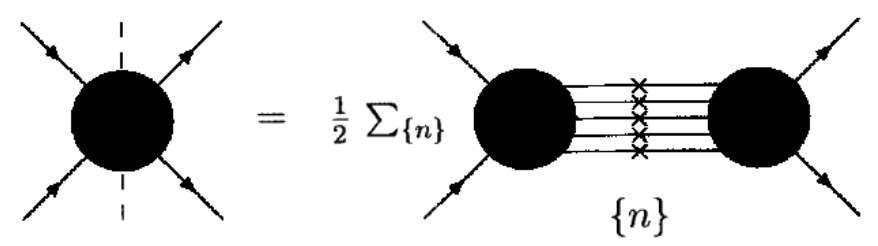

Figura 3.3: Diagrama da condição de unitariedade da amplitude de espalhamento $[106]$.

3.54 para o caso em que o estado final seja igual ao estado inicial. Asim:

$$
\sigma_{\text {total }}=\frac{2}{\Phi} \operatorname{Im} A_{\text {elas }}(s, t=0) \underset{s \rightarrow \infty}{\simeq} \frac{2}{s} \operatorname{Im} A_{\text {elas }}(s, t=0),
$$

então, a seção de choque total do processo de espalhamento depende unicamente do canal elástico, sem importar o número de canais inelásticos que poderão existir. De fato é a soma de todas as amplitudes dos canais inelásticos que "contrói" a amplitude do canal elástico. A condição de unitariedade sugere algumas suposições quando a energia do centro de massa do sistema ao quadrado $s$ se incrementa e com ela, o numero de canais aumentará até infinito $|n\rangle \rightarrow \infty$. Estas consequências são verificadas depois nos experimentos. Uma delas é que, desde que para altas energias a seção de choque deverá tender a um valor limite, a amplitude será predominantemente imaginária. Outra consequência se deriva do caráter randômico da fase da onda espalhada que permite um grande cancelamento da contribuição imaginária na amplitude a altas energias, assim, a seção de choque diminuirá abruptamente na medida em que a direção do vetor da onda espalhada se afaste da direção dianteira. 


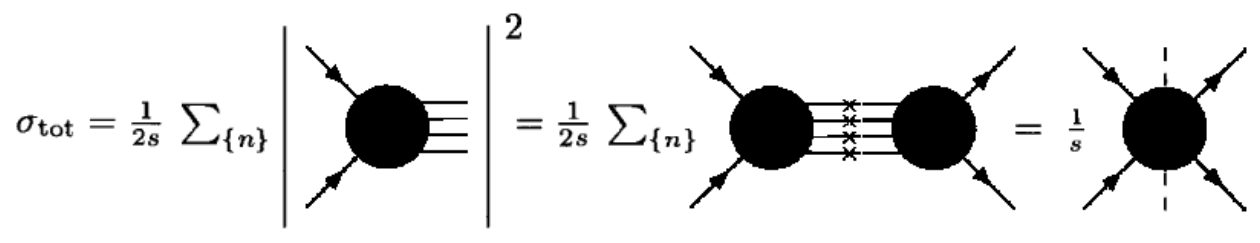

FIGURA 3.4: Representação gráfica do teorema óptico para o espalhamento relativística $[106]$.

\subsubsection{Analiticidade}

O postulado de analiticidade estabelece que os termos da matriz $S$ são funções analíticas que dependem das variáveis cinemáticas tratadas como números complexos. A amplitude física é o limite real para estas funções. Na mecânica quântica, a analiticidade está relacionada com a causalidade. É esperado que a conexão se mantenha na teoria de campo e na teoria da matriz $S$ relativística embora não haja prova desta tese. Para mostrar, não uma prova, mas um razoável argumento, vamos ver a amplitude da matriz $S$ não relativística expandida em termos da série de Born sem ter em conta a convergência desta.

Definindo $\boldsymbol{\Delta}=\left(\mathbf{k}-\mathbf{k}^{\prime}\right)^{2}=2 k^{2}(1-\cos \vartheta)$. A região fisicamente possível está definida para $-1 \leq \cos \vartheta \leq 1$. A amplitude pode ser expressa de duas maneiras:

$$
f\left(k^{2}, \cos \vartheta\right)=f\left(k^{2}, 1-\frac{\boldsymbol{\Delta}^{2}}{2 k^{2}}\right)=F\left(k^{2}, \boldsymbol{\Delta}^{2}\right) .
$$

Assim pode ser expandida:

$$
\begin{aligned}
& -4 \pi f\left(k^{2}, \cos \vartheta\right)=\left\langle k^{\prime}|V| k\right\rangle+\frac{1}{(2 \pi)^{3}} \int\left\langle k^{\prime}|V| p\right\rangle \frac{d^{3} p}{(k+i \varepsilon)^{2}-p^{2}}\langle p|V| k\rangle \\
& +\frac{1}{(2 \pi)^{6}} \int\left\langle k^{\prime}|V| p_{1}\right\rangle \frac{d^{3} p_{1}}{(k+i \varepsilon)^{2}-p_{1}^{2}}\left\langle p_{1}|V| p_{2}\right\rangle \frac{d^{3} p_{2}}{(k+i \varepsilon)^{2}-p_{2}^{2}}\left\langle p_{2}|V| k\right\rangle+\ldots
\end{aligned}
$$

Para o potencial de Yukawa:

$$
V(r)=g \frac{e^{-\mu r}}{r}
$$


e calculando o termo

$$
\begin{aligned}
\left\langle p|V| p^{\prime}\right\rangle & =\int e^{i\left(\mathbf{p}-\mathbf{p}^{\prime}\right) \mathbf{x}} \frac{e^{-\mu r}}{r} d^{3} x \\
& =2 \pi g \int_{0}^{\infty}\left\{\frac{e^{i\left|\mathbf{p}-\mathbf{p}^{\prime}\right| r}-e^{-i\left|\mathbf{p}-\mathbf{p}^{\prime}\right| r}}{i\left|\mathbf{p}-\mathbf{p}^{\prime}\right| r}\right\} e^{-\mu r} r d r \\
& =\frac{2 \pi g}{i\left|\mathbf{p}-\mathbf{p}^{\prime}\right|}\left\{\frac{1}{\mu-i\left|\mathbf{p}-\mathbf{p}^{\prime}\right|}-\frac{1}{\mu+i\left|\mathbf{p}-\mathbf{p}^{\prime}\right|}\right\} \\
& =\frac{4 \pi g}{\left(\mathbf{p}-\mathbf{p}^{\prime}\right)^{2}+\mu^{2}},
\end{aligned}
$$

a série de Born para a amplitude 3.68 pode ser escrita como:

$$
F\left(k^{2}, \Delta^{2}\right)=-\sum_{n=1}^{\infty} F_{n}\left(k^{2}, \Delta^{2}\right)
$$

e cada termo:

$$
\begin{aligned}
& F_{1}\left(k^{2}, \boldsymbol{\Delta}^{2}\right)=\frac{4 \pi g}{\boldsymbol{\Delta}^{2}+\mu^{2}} \\
& F_{2}\left(k^{2}, \boldsymbol{\Delta}^{2}\right)=\frac{2 g^{2}}{\pi} \int \frac{d \mathbf{p}}{\left[\left(\mathbf{k}^{\prime}-\mathbf{p}\right)^{2}+\mu^{2}\right]\left[\mathbf{k}^{2}-\mathbf{p}^{2}+i \varepsilon\right]\left[(\mathbf{k}-\mathbf{p})^{2}+\mu^{2}\right]} \\
& F_{3}\left(k^{2}, \boldsymbol{\Delta}^{2}\right)=\frac{g^{3}}{\pi^{3}} \iint \frac{1}{\left[\left(\mathbf{k}^{\prime}-\mathbf{p}_{1}\right)^{2}+\mu^{2}\right]\left[\mathbf{k}^{2}-\mathbf{p}_{1}^{2}+i \varepsilon\right]\left[\left(\mathbf{p}_{1}-\mathbf{p}_{2}\right)^{2}+\mu^{2}\right]} \\
& \times \frac{d \mathbf{p}_{1} d \mathbf{p}_{2}}{\left[\mathbf{k}^{2}-\mathbf{p}_{2}^{2}+i \varepsilon\right]\left[\left(\mathbf{p}_{2}-\mathbf{k}\right)^{2}+\mu^{2}\right]} .
\end{aligned}
$$

As propriedades analíticas dos temos da série da amplitude em funçaõ da variável $\boldsymbol{\Delta}$ (ou correspondente ao momento transferido) poderão ser analizadas das equações anteriores e assim estudar seu significado físico. No primeiro termo a singularidade é um pólo simples devido à forma do potencial de Yukawa $\Delta^{2}=-\mu$. A posição do pólo é determinado pelo alcance do potencial: quanto maior o alcance, menor será o valor de $\mu$. Para o caso $\mu=0$ (potencial de Coulomb) o sistema atinge um dos limites da região física $\cos \vartheta=1$. Para analisar os outros termos da expansão é importante considerar seu sentido físico: o termo $F_{n}$ corresponde a um espalhamento $n$-vezes sucessivo pelo potencial. Pode-se inferir que o primeiro termo está associado a um espalhamento onde a partícula é espalhada com o momento transferido fisicamente impossível $\boldsymbol{\Delta}=-\mu^{2}$. Se uma partícula é $n$ vezes espalhada significa que passou por $n$ processos de espalhamento com momento transferido $i \mu$. No segundo espalhamento as direções dos dois espalhamentos coincidem na fronteira do cone e corresponde a um valor de momento transferido $2 i \mu$ e a uma singularidade em $\boldsymbol{\Delta}^{2}=4 \mu^{2}$. Assim o termo $F_{n}$ terá uma singularidade em $\boldsymbol{\Delta}^{2}=n^{2} \mu^{2}$. Ainda existe mais um tipo de singularidade que é dada pela soma das contribuições entre um espalhamento duplo com momento transferido -i $i$ com um ângulo de espalhamento $\vartheta$ e um espalhamento simples com momento transferido $-2 i \mu$ com ângulo $2 \vartheta$ como é 


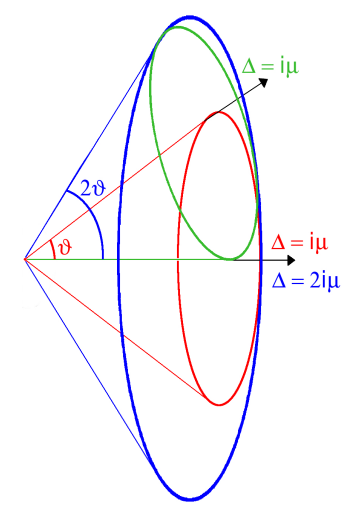

FiguRA 3.5: Representação gráfica do espalhamento sucessivo por um potencial.

mostrado na figura 3.5. Por exemplo para o segundo termo da série existiriam duas singularidades:

$$
\begin{aligned}
& \boldsymbol{\Delta}_{1}=-4 \mu^{2} \\
& \boldsymbol{\Delta}_{2}=-4 \mu^{2}-\frac{\mu^{2}}{k^{2}}
\end{aligned}
$$

onde foi usada a definição $\boldsymbol{\Delta}_{2}=\left(\mathbf{k}-\mathbf{k}^{\prime}\right)^{2}=2 k^{2}(1-\cos 2 \vartheta)$ :

$$
\cos \vartheta=1+\frac{\mu^{2}}{2 k^{2}}, \cos 2 \vartheta=2 \cos ^{2} \vartheta-1=1+\frac{2 \mu^{2}}{k^{2}}+\frac{\mu^{4}}{2 k^{4}}
$$

Desde o ponto de vista relativística, a amplitude de espalhamento tem singularidades de tipo pólo simples e branch points. O primeiro tipo de singularidade se refere à troca de uma partícula, enquanto o segundo à troca de duas ou mais partículas. Um exemplo do anterior é que para o espalhamento duas partículas a amplitude $A(s, t)$ tem as diferentes singularidades pela troca de partículas de massa $m$ :

$$
\begin{aligned}
s & =m^{2} \text { polo simple do canal } s \\
s & =(2 m)^{2},(3 m)^{2},(4 m)^{2} \ldots \text { branch point canal } s \\
t & =m^{2} \text { polo simple do canal } t \\
t & =(2 m)^{2},(3 m)^{2},(4 m)^{2} \ldots \text { branch point canal } t \\
u & =m^{2} \text { polo simple do canal } u \\
u & =(2 m)^{2},(3 m)^{2},(4 m)^{2} \ldots \text { branch point canal } u .
\end{aligned}
$$

Os branch points $s=(2 m)^{2}, s=(3 m)^{2}$ são mostrados na Figura 3.6

Um pólo simples pode ser considerado na teoria perturbativa como um processo onde é trocada uma partícula escalar. 

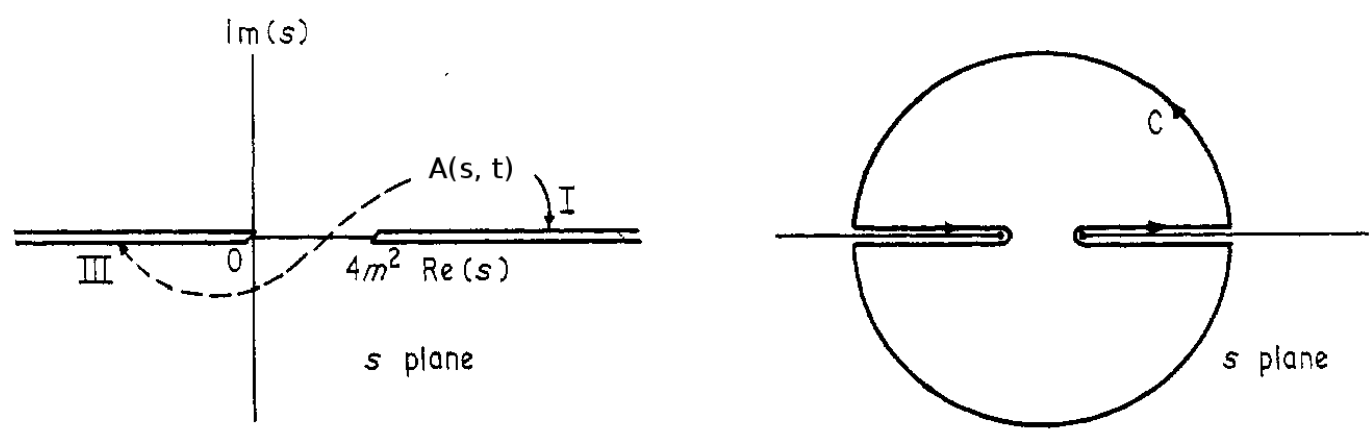

Figura 3.6: Singularidades da matriz de espalhamento frontal de duas partículas [106].

\subsubsection{Crossing}

Propriedade muito importante na teoria é o "crossing", que se refere a relações entre processos de espalhamento que envolvem as mesmas partículas. Está baseado na propriedade da teoria relativística de campos onde uma partícula incidente pode ser vista como uma antipartícula espalhada de momento $-p$. Esta propriedade relaciona os canais $s, t, u$ da forma

$$
\begin{array}{rr}
\text { I : } & 1+2 \rightarrow 3+4 \quad \text { canal } s \\
\text { II }: & 1+\overline{3} \rightarrow \overline{2}+4 \quad \text { canal } t \\
\text { III }: & 1+\overline{4} \rightarrow \overline{2}+3 \quad \text { canal } u
\end{array}
$$

O postulado da simetria do crossing na teoria da matriz $S$ relativística afirma que os três processos são descritos pela mesma amplitude. Está baseado no fato de que as regiões cinemáticas dos três canais são diferentes e não se sobrepõem, assim a amplitude expressa nas variáveis de Mandelstam descreve as reações 3.80 em diferentes domínios destas variáveis.

Usando $s$ e $t$ como variáveis independentes e fixada $t$, as singularidades no canal $u$ da forma das Equações 3.79 da amplitude aparecem no plano $s$ nos pontos

$$
\begin{aligned}
& s=3 m^{2}-t \text { pólo simples } \\
& s=-t, s=-t-5 m^{2}, \text { etc. branch point }
\end{aligned}
$$

onde é usada a relação

$$
s+t+u=4 m^{2} .
$$


Devido ao principio de Schwarz, o caráter real da amplitude para $-t<s<4 m^{2}$ implica

$$
A\left(s^{*}, t\right)=A^{*}(s, t) \text {. }
$$

A relação anterior define a descontinuidade da amplitude

$$
D_{s}(s, t) \equiv \operatorname{Disc}_{s} A(s, t, u)=\frac{1}{2 i} \lim _{\varepsilon \rightarrow 0^{+}}[A(s+i \varepsilon, t)-A(s-i \varepsilon, t)],
$$

que é usada para a obtenção das relações de espalhamento da amplitude que são fundamentais para a formulação da teoria de Regge.

As relações de espalhamento são obtidas a partir da representação integral de Cauchy da amplitude

$$
A(s, t)=\frac{1}{2 \pi i} \oint_{\Gamma} \frac{A\left(s^{\prime}, t\right)}{s^{\prime}-s} d s^{\prime}
$$

onde $\Gamma$ é o contorno em sentido contrario do relógio no plano $s$ e é a soma $\Gamma=\gamma_{1}+$ $\gamma_{2}+\Gamma_{1}+\Gamma_{2}+C$ assegurando assim o caráter analítico da amplitude como é mostrado na Figura 3.7. É logico supor que a amplitude se desfaze para grandes valores de $s$

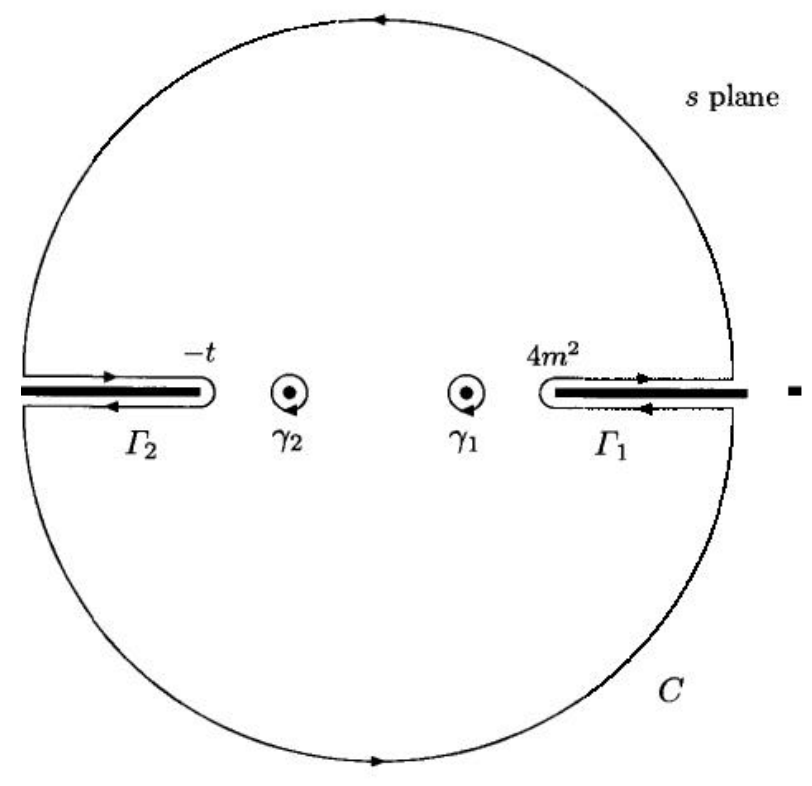

Figura 3.7: Contorno da integral de Cauchy da amplitude $A(s, t)[106]$

$$
A(s, t) \underset{|s| \rightarrow \infty}{\rightarrow \rightarrow \infty} 0,
$$

Esta suposição faz que a integral pela trajetória onde $R_{|s|} \rightarrow \infty$ é zero. Usando o teorema do resíduo, a definição de descontinuidade e assumindo a condição 3.86, a amplitude pode 
ser expressa como

$$
A(s, t)=\frac{1}{\pi} \int_{4 m^{2}}^{\infty} \frac{D_{s}\left(s^{\prime}, t\right)}{s^{\prime}-s} d s^{\prime}+\int_{-\infty}^{-t} \frac{D_{s}\left(s^{\prime}, t\right)}{s^{\prime}-s} d s^{\prime}+\text { polos } \ldots
$$

Esta expressão é conhecida como relação de espalhamento da amplitude de uma variável, ou seja, a variável $t$ é fixada. É importante notar que a amplitude pode ser definida totalmente usando os critérios de analiticidade e unitariedade. Na realidade, a condição 3.86 não é cumprida. O que se cumpre é que no limite para grandes valores da energia de centro de massa ao quadrado a amplitude tende a uma potência de $s$

$$
A(s, t)_{|s| \rightarrow \infty}^{\rightarrow} s^{\lambda}
$$

onde $\lambda$ é um numero real positivo. Devido a isto, para eliminar o termo da integral para a trajetória $R_{|s|} \rightarrow \infty$ a relação de espalhamento deverá ser escrita para a quantidade

$$
\frac{A(s, t)}{\left(s-s_{1}\right)\left(s-s_{2}\right) \ldots\left(s-s_{N}\right)},
$$

onde $N$ é o menor número inteiro maior que $\lambda$ e as substrações $s_{1}, s_{2} \ldots . . s_{N}$ são constantes arbitrárias. A relação de espalhamento fica

$$
\begin{aligned}
A(s, t) & =\sum_{n=0}^{N-1} c_{n}(t) s^{n} \\
& +\frac{1}{\pi}\left(s-s_{1}\right)\left(s-s_{2}\right) \ldots\left(s-s_{N}\right) \int_{4 m^{2}}^{\infty} \frac{D_{s}\left(s^{\prime}, t\right)}{\left(s^{\prime}-s_{1}\right)\left(s^{\prime}-s_{2}\right) \ldots\left(s^{\prime}-s_{N}\right)} d s^{\prime} \\
& +\frac{1}{\pi}\left(u-u_{1}\right)\left(u-u_{2}\right) \ldots\left(u-u_{N}\right) \int_{4 m^{2}}^{\infty} \frac{D_{u}\left(u^{\prime}, t\right)}{\left(u^{\prime}-u_{1}\right)\left(u^{\prime}-u_{2}\right) \ldots\left(u^{\prime}-u_{N}\right)} d u^{\prime} \\
& + \text { polos... }
\end{aligned}
$$

onde foi usada a relação cinématica $u_{i}=4 m^{2}-s_{i}-t$. Esta relação de espalhamento é conhecida como relação de espalhamento substraída $\mathrm{N}$ vezes e está indeterminada pelo polinômio $\sum_{n=0}^{N-1} c_{n}(t) s^{n}$, que não pode ser determinado a priori.

Se a seção de choque é assintoticamente constante como é esperado na teoria de Regge

$$
\sigma_{t o t} \rightarrow \text { const, }
$$

de acordo com o teorema óptico mostrado na Eq. 3.66, a parte imaginária da amplitude de espalhamento na direção dianteira tem uma dependência linear com $s$

$$
\operatorname{Im} A_{e l}(s, 0) \underset{s \rightarrow \infty}{\rightarrow} s
$$


Então, só os dois primeiros termos do polinômio da relação de dispersão 3.90 são necessários para definir a amplitude, ou o que é o mesmo, dois constantes $c_{0}(0)$ e $c_{1}(0)$. Uma representação importante desta relação é a representação de Froissart-Gribov escrita em função das ondas parciais. Se a amplitude para um valor de $t$ fixo cumpre

$$
A(s, t) \underset{s \rightarrow \infty}{\rightarrow} s^{\lambda},
$$

usando o ângulo de espalhamento $\vartheta$

$$
z_{t} \equiv \cos \vartheta_{t}=1+\frac{2 s}{t-4 m^{2}}=-\left(1+\frac{2 u}{t-4 m^{2}}\right)
$$

a condição 3.93 tem a forma

$$
A\left(z_{t}, t\right) \underset{z_{t} \rightarrow \infty}{\rightarrow} z_{t}^{\lambda}, .
$$

A amplitude pode ser expandida em ondas parciais da forma

$$
A_{l}(t)=\frac{1}{2} \int_{-1}^{1} d \cos \vartheta_{t} P_{l}\left(\cos \vartheta_{t}\right) A\left(s, \cos \vartheta_{t}\right)
$$

onde $\vartheta_{t}$ é o ângulo de espalhamento no canal $t$. Expressando a relação de espalhamento em função do ângulo $\vartheta_{t}$ e em ondas parciais

$$
\begin{aligned}
A_{l}(t) & =\frac{1}{2} \sum_{n=0}^{N-1} c_{n}(t) \int_{-1}^{1} d z_{t} P_{l}\left(z_{t}\right) z_{t}^{n} \\
& +\frac{1}{2 \pi} \int_{-1}^{1} d z_{t} z_{t}^{N} P_{l}\left(z_{t}\right) \int_{z_{0}}^{\infty} \frac{D_{s}\left(s^{\prime}\left(z_{t}, t\right), t\right)}{z_{t}^{\prime N}\left(z_{t}^{\prime}-z_{t}\right)} d z_{t}^{\prime} \\
& +\frac{1}{2 \pi} \int_{-1}^{1} d z_{t} z_{t}^{N} P_{l}\left(z_{t}\right) \int_{-z_{0}}^{-\infty} \frac{D_{u}\left(u^{\prime}\left(z_{t}, t\right), t\right)}{z_{t}^{\prime N}\left(z_{t}^{\prime}-z_{t}\right)} d z_{t}^{\prime}
\end{aligned}
$$

onde $z_{0}$ é o branch point no canal $t$ e as subtrações $z_{1}=0, z_{2}=0 \ldots z_{N}=0$ por simplicidade. Para $l \geq N$ o primeiro termo da equação 3.97 é zero pelas propriedades dos harmônicos esféricos. Desta forma a representação de Froissart-Gribov para a amplitude expandida em ondas parciais fica da forma

$$
A_{l}(t)=\frac{1}{\pi} \int_{z_{0}}^{\infty} d z_{t} D_{s}\left(s^{\prime}\left(z_{t}, t\right), t\right) Q_{l}\left(z_{t}\right)+\frac{1}{\pi} \int_{z_{0}}^{\infty} d z_{t} D_{u}\left(u^{\prime}\left(z_{t}, t\right), t\right) Q_{l}\left(z_{t}\right)
$$

onde $Q_{l}\left(z_{t}\right)$ é a função de Legendre de secunda ordem

$$
Q_{l}\left(z_{t}\right)=\frac{1}{2} \int_{1}^{-1} d z_{t} P_{l}\left(z_{t}\right) \frac{z_{t}^{N}}{z_{t}^{\prime N}\left(z_{t}^{\prime}-z_{t}\right)}
$$

Desta representação são demostrados importantes teoremas que serão brevemente expostos, mas suas demonstrações poderão ser encontradas nos artigos originais. 
Teorema de Froissart-Martin $[111,112]$. Este teorema determina que a seção de choque não pode crescer mais rápido que $\ln ^{2} s$, ou seja:

$$
\sigma_{\text {total }} \leq C \ln ^{2} s, \text { para } s \rightarrow \infty,
$$

colocando assim um limite estrito ao crescimento da seção de choque de qualquer processo.

Teoremas de Pomeranchuk [113]. Este teorema estabelece que as seções de choque totais das colisão alvo-partícula $(a b)$ e alvo-antipartícula $(a \bar{b})$ são assintoticamente iguais, ou seja:

$$
\sigma_{\text {total }}(a b) \underset{s \rightarrow \infty}{\stackrel{\simeq}{\rightarrow}} \sigma_{\text {total }}(a \bar{b})
$$

Este teorema está baseado no fato que os canais de que diferenciam uma colisão da outra se fecham nas altas energias e.g. a seção de choque total da colisão $p \bar{p}$ decresce assintoticamente até a seção de choque total de $p p$ como é mostrado na figura 3.8. O teorema abre a possibilidade de escrever novas relações de dispersão entre amplitudes de espalhamento.

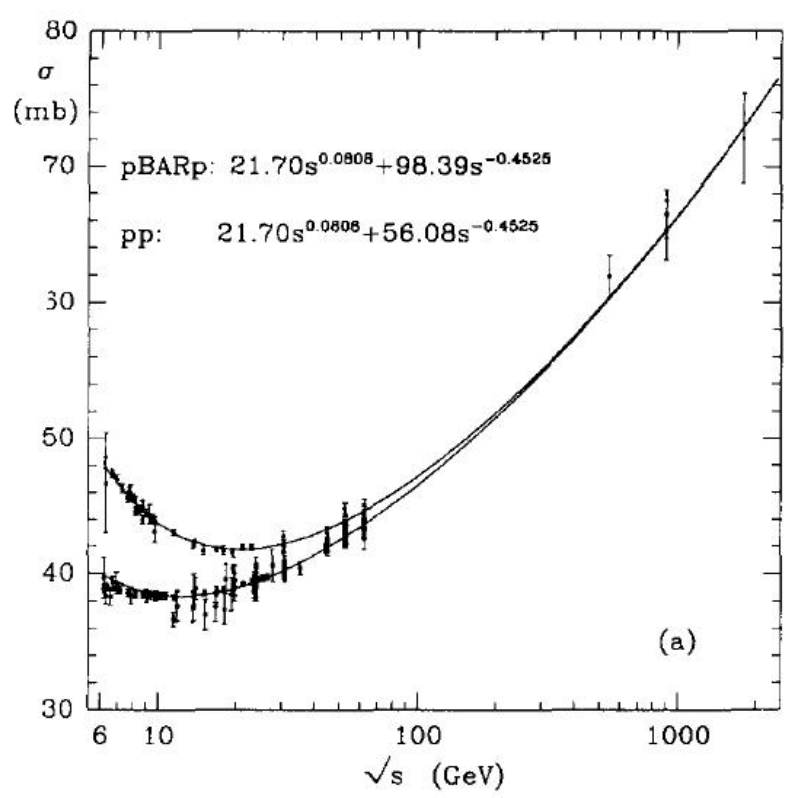

Figura 3.8: Seções de choque totais das colisões $p p$ e $p \bar{p}$ ajustadas por A. Donnachie e P. V. Landshoff usando a teoría de Regge que demostram o teorema de Pomeranchuk [114]. 


\subsection{Teoria de Regge}

A teoria de Regge propõe a existência de funções complexas $\alpha_{n}(s)$, onde $s$ é a energia do centro de massa ao quadrado, definidas como trajetórias de Regge que relacionan diferentes partículas ou ressonâncias. Estas funções cumprem $\operatorname{Re} \alpha_{n}(s)=J($ spin da partícula) para $s=m_{n}^{2}$. Na teoria relativística as partículas relacionadas tem os mesmos números quânticos (isospin, paridade, estranheza, etc..) mas os spins diferem em 2 unidades de $\hbar$. No espalhamento por um potencial não relativística, as trajetórias relacionan estados ligados ou ressonn̂acias que diferem no spin em unidades de $1 \hbar$, se as forças de troca não são levadas em conta. Seja o exemplo ${ }^{2}$ mostrado na Figura 3.9: o próton ou nêutron, $\mathrm{N}(936)$, com valor de spin $\frac{1}{2}$, isospin $\frac{1}{2}$ e paridade + , está relacionado com as ressonâncias $\mathrm{N}(1688)$ e $\mathrm{N}(2220)$ no sistema $\pi N$ que tem o mesmo isospin e paridade que o nucleon, mas com valores de spin $\frac{5}{2}$ e $\frac{9}{2}$ respectivamente. Outro exemplo são as ressonâncias $\Delta(1236), \Delta(1950)$ e $\Delta(2420)$, todas com os mesmos valores de isospin $\frac{3}{2}$ e paridade + , número bariônico 1 e estranheza 0 , mas com valores de spin $\frac{3}{2}, \frac{7}{2}$ e $\frac{11}{2}$ respectivamente. As trajetórias são muito bem ajustes por uma recta, em primeira

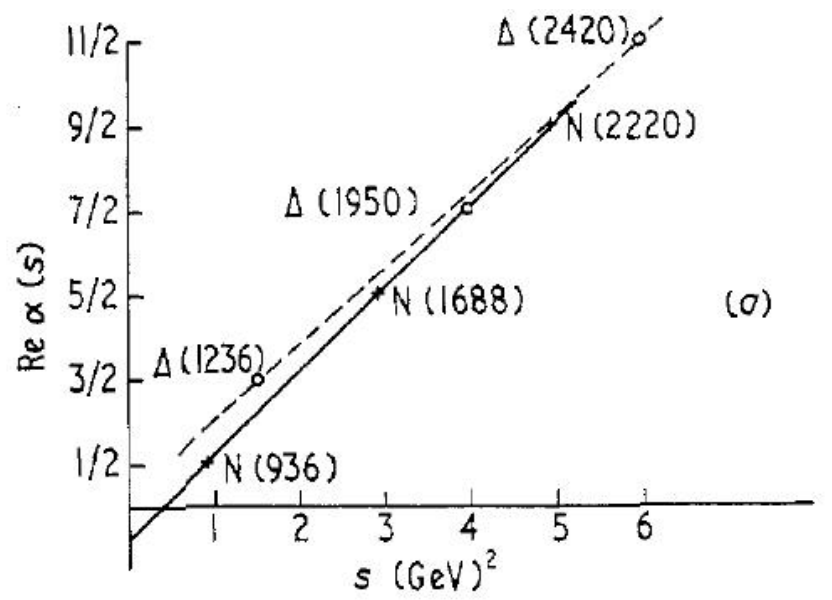

Figura 3.9: Gráfico das trajetórias da partículas $\mathrm{N}$ e $\Delta$ [106].

aproximação:

$$
\alpha(s)=\alpha_{0}+\alpha^{\prime} s
$$

onde $\alpha_{0}$ e $\alpha^{\prime}$ são o ponto de intercepção e a tangente da reta respectivamente. Experimentalmente se sabe que a inclinação da reta tem o valor único $\alpha^{\prime} \sim 1$ para todas as famílias de partículas.

Outra proposta da teoria de Regge é a relação entre estas trajetórias e o comportamento dos processos de espalhamento nas altas energias. Esta expõe que o processo de espalhamento a altas energias é definido pela troca de um objeto virtual chamado Reggeon

\footnotetext{
${ }^{2} \mathrm{O}$ spin é expresso em unidades de $\hbar$.
} 
associado a uma trajetória $\alpha_{n}(t)$. O Reggeon não é uma partícula física. Pode ser demonstrado que se a partícula trocada é um méson, o processo violaria o limite de Froissart-Martin [106] . Esta trajetória $\alpha_{n}(t)$ do canal $t$ corresponde à ressonância que é formada pelos os estados iniciais e finais das partículas deste canal. Exemplo: seja a reação no canal $s$ :

$$
\pi^{-} p \rightarrow \pi^{0} n
$$

no canal $t$ é da forma:

$$
p n \rightarrow \pi^{-} \pi^{0}
$$

A seção de choque elástica para a reação $\pi^{-} \pi^{0}$ têm associada a sim uma ressonância nos $765 \mathrm{MeV}$ que é a partícula $\rho^{+}$. Esta pode estar associada também ao estado ligado do sistema $n p$. De acordo a teoria há uma trajetória de Regge $\alpha_{\rho}(t)$ associada a esta ressonância que depende do valor do momento transferido ao quadrado $t$ como é mostrado na Figura 3.10. A colisão estará dominada pela troca do Reggeon com maior

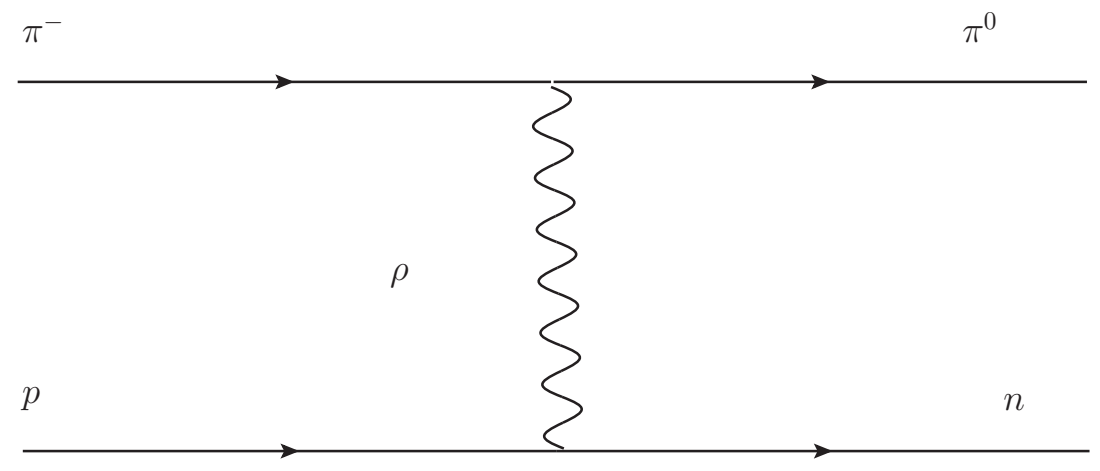

FigurA 3.10: Diagramas da troca do Reggeon $\rho$ na colisão $\pi^{-} p \rightarrow \pi^{0} n$.

valor da parte real da trajetótia $\operatorname{Re}\left[\alpha_{n}(t)\right]$. A teoria de Regge calcula uma seção de choque diferencial para esta reação da forma:

$$
\frac{d \sigma}{d t} \sim|b(t)|^{2} s^{2 \alpha(t)-2},
$$

onde, para altas energias, $|b(t)|$ pode ser considerado constante. Substituindo a expressão 3.102 para $\alpha(t)$ e usando os valores $\alpha_{\rho}^{0}=1 / 2$ e $\alpha_{\rho}^{\prime}=1$, a seção de choque diferencial fica:

$$
\frac{d \sigma\left(\pi^{-} p \rightarrow \pi^{0} n\right)}{d t} \sim\left|b_{0}\right|^{2} s^{2 \alpha_{\rho}^{0}-2} \exp \left(2 \alpha_{\rho}^{\prime} t \ln s\right) .
$$

Integradando em $t$ é obtida a seção de choque total:

$$
\sigma\left(\pi^{-} p \rightarrow \pi^{0} n\right) \sim \frac{\left|b_{0}\right|^{2}}{s 2 \alpha_{\rho}^{\prime} \ln s} .
$$


Este resultado está de acordo com dados experimentais [115] que confirmam o valor $\alpha_{\rho}^{0} \sim m_{\rho^{+}}^{2} \sim 0.5$. e $\alpha_{\rho}^{\prime} \sim 1$.

\subsection{Abordagem matemática da teoria de Regge.}

Nesta seção será tratado brevemente a abordagem matemática em que está baseada a teoria de Regge. Aqui serão expostos inicialmente propriedades matemáticas da amplitude expandida em harmônicos esféricos que depois será usada na teoria de espalhamento a altas energias a partir dos resultados anteriores.

A teoria tem como base a amplitude expandida em ondas parciais no canal $s$ :

$$
\begin{aligned}
A(s, z) & =\sum_{l=0}^{\infty} A_{l}(s) P_{l}(z) \\
A_{l}(s) & =\frac{1}{2} \int_{-1}^{1} A(s, z) P_{l}(s, t(s, z)) d z .
\end{aligned}
$$

onde:

$$
z \equiv \cos \vartheta=1+\frac{2 t}{s-4 m^{2}}
$$

Esta série converge se:

$$
-1 \leq z \leq 1 \text { e } s \geq 4 m^{2}
$$

Pode-se demostrar que a relação anterior implica que $z$ está dentro de uma elipse com foco -1 e 1, no plano complexo, chamada elipse de Lehmann. Este critério de convergência está correto para energias baixas, mas para energias relativísticas o $\cos \vartheta$ pode ter valores maiores do que 1 uma vez que a Equação 3.110 limita o domínio das variáveis de Mandelstam $t$ e $u$ para um valor fixo de $s$. Para que a série consiga convergir para maiores valores de $z$, Regge expande a amplitude $A(s, z)$ usando uma transformação de Sommerfeld-Watson e extrapolando o momento angular $l$ ao plano dos números complexos. Neste caso a amplitude pode ser escrita como:

$$
\begin{aligned}
A(s, z) & =-\sum_{i} \pi\left(2 \alpha_{i}(s)+1\right) \beta_{i}(s) \frac{P_{\alpha_{i}}(-z)}{\sin \pi \alpha_{i}} \\
& -\frac{1}{2 i} \int_{c-i \infty}^{c+i \infty} d l(2 l+1) A(l, s) \frac{P_{l}(-z)}{\sin \pi l}
\end{aligned}
$$

onde $\alpha_{i}(s)$ é a localização dos pólos no plano complexo do momento angular $l, \beta_{i}(s)$ é o resíduo da integral e $-\frac{1}{2} \leq \operatorname{Re} c<0$. A integral $\int_{c-i \infty}^{c+i \infty}$ vai ao longo de uma reta paralela 
ao eixo imaginário de $l$. A equação 3.112 é conhecida como a representação SommerfeldWatson da amplitude e é a ferramenta fundamental para o abordagem teórica do momento angular complexo. Usando esta representação se expande o domínio das variáveis $t$ e $u$ para uma energia do centro de massa ao quadrado $s$. Os termos $P_{l}(z)$ obedecem a relação:

$$
P_{l}(z) \underset{z \rightarrow \infty}{\sim}|z|^{\operatorname{Re} \alpha} \sim|z|^{l}
$$

Como o termo integral da representação Sommerfeld-Watson da amplitude 3.112 se comporta como $|z|^{-\frac{1}{2}}$ no limite $|z| \rightarrow \infty$, a única contribuição neste limite será a contribuição dos pólos, assim:

$$
A(s, t) \underset{t \rightarrow \infty}{\sim}-\beta(s) \frac{t^{\alpha(s)}}{\sin \pi \alpha(s)}
$$

ou:

$$
A(s, t) \underset{s \rightarrow \infty}{\sim}-\beta(t) \frac{s^{\alpha(t)}}{\sin \pi \alpha(t)}
$$

se for expandindo em ondas parciais no canal $t$ ao inicio. Este resultado está de acordo com o princípio de crossing e mostra que a singularidade principal é a que define o comportamento da amplitude de espalhamento a altas energias. As equações 3.114 e 3.115 são válidas mesmo para correções sub-assintóticas dadas pela inclusão de pólos menores e outras singularidades que alteram seu comportamento produzindo, por exemplo, um comportamento tipo $\ln s$.

A forma da Equação 3.114 para a amplitude pode ser obtida na mecânica quântica usando potenciais de Yukawa e na teoria de espalhamento relativística. Nesta última abordagem é preciso definir duas amplitudes para cumprir o princípio de crossing. A partir das equações da teoria relativística descrita acima em 3.97, estas tem a forma:

$$
A_{l}^{ \pm}(t)=\frac{1}{\pi} \int_{z_{0}}^{\infty}\left[D_{s}(s, t) \pm D_{u}(s, t)\right] Q_{l}\left(z_{t}\right) d z_{t},
$$

onde:

$$
\begin{array}{lllll}
A_{l}^{+}(t)=A_{l}(t) & \text { para } & l & \text { par } \\
A_{l}^{-}(t)=A_{l}(t) & \text { para } & l & \text { impar. }
\end{array}
$$

Introduzindo a variável $\xi$ com dois possíveis valores \pm 1 , a amplitude pode ser escrita como:

$$
A_{l}(t)=\frac{1}{2} \sum_{\xi= \pm 1}\left(1+\xi \mathrm{e}^{-i \pi l}\right) A_{l}^{\xi}(t) .
$$


Em altas energias têm a forma:

$$
A(s, t) \underset{s \rightarrow \infty}{\sim}-\beta(t) \frac{1+\xi \mathrm{e}^{-i \pi l}}{\sin \pi \alpha(t)} s^{\alpha(t)}
$$

Se o análise tivesse sido expandindo inicialmente a amplitude em ondas parciais no canal $s$ o resultado para grandes valores de momento transferido é

$$
A(s, t) \underset{t \rightarrow \infty}{\sim}-\beta(s) \frac{1+\xi \mathrm{e}^{-i \pi l}}{\sin \pi \alpha(s)} t^{\alpha(s)} .
$$

Nesta expressão, o limite $s \rightarrow \infty$ é aplicado no final, mas para pode ser aplicado antes para se-obter a representação de Sommerfeld-Watson. O anterior é possível se é usada as propriedades dos polinômios de Legendre de primeira e segunda ordem:

$$
\begin{aligned}
P_{l}(z) \underset{|z| \rightarrow \infty}{\sim} \frac{1}{\sqrt{\pi}} \frac{\Gamma\left(l+\frac{1}{3}\right)}{\Gamma(l+1)}(2 z)^{l} \\
Q_{l}(z)_{|z| \rightarrow \infty}^{\sim} \sqrt{\pi} \frac{\Gamma(l+1)}{\Gamma\left(l+\frac{2}{3}\right)}(2 z)^{-l-1} .
\end{aligned}
$$

Substituindo estas últimas, a amplitude tem a forma:

$$
A(s, t)=-\frac{1}{4 \pi i} \sum_{\xi \pm 1} \int_{c-i \infty}^{c+i \infty} \frac{\xi+\mathrm{e}^{-i \pi l}}{\sin \pi l}\left(\frac{s}{|t|}\right)^{l} a^{\xi}(l, t) d l
$$

onde $a^{\xi}(l, t)$ é a transformada de Mellin da descontinuidade $D_{l}^{\xi}(s, t)$. O comportamento da amplitude dependerá desta quantidade já que é esta a que tem incluída as singularidades no plano complexo de $l$. Exemplo desta dependência pode ser vista nas relações:

$$
\begin{aligned}
D_{s}^{\xi}(s, t) \sim s^{\alpha} & \Rightarrow \quad a(l, t) \sim \frac{1}{l-\alpha} \\
D_{s}^{\xi}(s, t) \sim s^{\alpha}(\ln s)^{-1} & \Rightarrow \quad a(l, t) \sim \ln (l-\alpha) .
\end{aligned}
$$

Na primeira a amplitude tem um pólo simples da mesma forma que se comporta na teoria de Regge, entretanto o segundo exemplo mostra uma amplitude que tem um cut em vez de um pólo.

Vejamos agora a relação das trajetórias de Regge e as ressonâncias e os estados ligados. Na presença de um pólo de Regge a amplitude tem a forma

$$
A(l, t) \underset{l \rightarrow \alpha(t)}{\sim} \frac{\beta(t)}{l-\alpha(t)} .
$$


Suponhamos que para um número real $t_{0}$ exista $\alpha^{\prime}\left(t_{0}\right)$ definido por:

$$
t_{0}=l+\epsilon
$$

onde $l$ é um inteiro e $\epsilon$ e um número real pequeno comparado com 1. Expandindo $\alpha\left(t_{0}\right)$ na vicindade de $t_{0}$

$$
\alpha(t)=t_{0}+\alpha^{\prime}\left(t_{0}\right)\left(t-t_{0}\right)+\cdots=l+i \epsilon+\alpha^{\prime}\left(t_{0}\right)\left(t-t_{0}\right)+\ldots
$$

Substituindo esto no denominador da equação 3.127, esta fica da forma:

$$
A(l, t) \underset{l \rightarrow \tilde{\alpha}(t)}{\sim} \frac{\beta(t)}{t-t_{0}+i \Gamma} .
$$

onde:

$$
\Gamma=\frac{\operatorname{Im} \alpha\left(t_{0}\right)}{\alpha^{\prime}\left(t_{0}\right)}=\frac{\epsilon}{\alpha^{\prime}\left(t_{0}\right)},
$$

que tem a típica estrutura de uma ressonância de Breit-Wigner de massa $M=\sqrt{t_{0}}$. Embaixo do limiar para $t\left(4 m^{2}\right.$ para massa iguais) se cumpre $\operatorname{Im} \alpha(t)=0$, então os pólos de Regge corresponderam a estados ligados. Esto conclui que as trajetórias de Regge interpolam as famílias de estados ligados e ressonâncias com os mesmos números quânticos e se diferem pelo valor de momento angular $l=\operatorname{Re} \alpha(t)$ i.e. spin. O denominador da amplitude 3.127 se anula para qualquer valor inteiro de $l$, mas o numerador se anula para valores alternados de $l$ de acordo com seu assinatura $\xi$. Assim as trajetórias com assinatura positivo $\xi=1$ interpolarão ressonâncias e estados ligados com momento angular $l$ par enquanto que as trajetórias $\operatorname{com} \xi=-1$ interpolarão ressonâncias e estados ligados com momento angular $l$ ímpar.

O conceito principal a lembrar na teoria de Regge é que o comportamento assintótico da amplitude 3.114 no canal $s$ é a dado pela troca de trajetórias $\alpha(t)$, ou o que é o mesmo, famílias de ressonâncias no canal $t$. Entender esto ajuda a entender a natureza e o papel das singularidade do momento angular complexo. A ideia da troca de uma partícula em procesos e a existência do méson não é nova desde que foi abordado previamente por Yukawa. A novidade aqui é que esta troca de partículas virtuais acontece agora para o comportamento assintótico da reação. Quase inesperadamente as trajetórias $\alpha(t)$ ajustam a equação linear para todo o intervalo de valores de $t$ :

$$
\alpha(t)=\alpha(0)+\alpha^{\prime} t
$$

onde $\alpha(0)$ e $\alpha^{\prime}$ são os pontos de interseçaõ e a inclinação da recta respectivamente.

A inclinação das trajetórias tem o valor $\alpha^{\prime} \sim 1 \mathrm{GeV}^{-2}$. Nas trajetórias mesônicas o valor do ponto de interseção é aproximadamente $\alpha(0) \simeq \frac{1}{2}$ enquanto para as trajetórias 
bariônica o valor é bem bem menor. Existe uma propriedade associada às trajetórias mesônicas e é o fato de serem degeneradas. Assim cada uma das trajetórias mesônicas interpola ressonâncias com spin par e ímpar ao mesmo tempo. Na pratica as trajetórias com assinatura $\xi=1\left(f_{2}\right.$ e $\left.a_{2}\right)$ são indistinguíveis das de assinatura $\xi=-1$ ( $\rho$ e $\omega$ ) como é mostrado na interpolação destas na Figura 3.11.

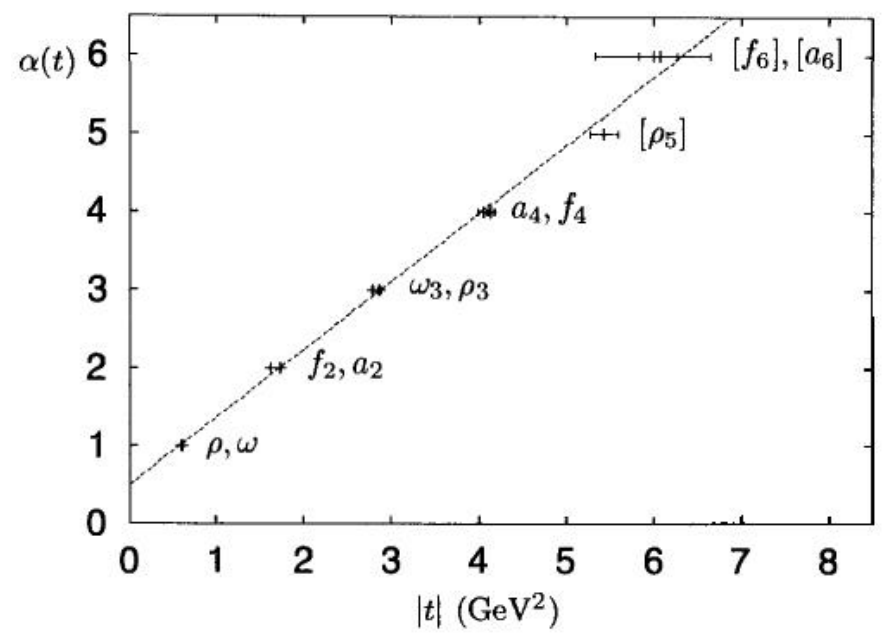

FIGURA 3.11: Interpolação da trajetória mesônica para las partículas $f_{2}, a_{2}, \rho, \omega$ [106].

Desde o ponte de vista fenomenológico é melhor reescrever a amplitude da forma:

$$
A(s, t)=\beta(t) \eta(t) s^{\alpha(t)}
$$

onde $\beta(s)$ é o resíduo e $\eta(t)$ é o fator de sinal:

$$
\eta(t)=-\frac{1+\xi \mathrm{e}^{-i \pi \alpha(t)}}{\sin \pi \alpha(t)}
$$

Para uma trajetória linear e desconsiderando a dependência de $t$ no denominador, mas para pequenos valores de $t$, o último termo da equação 3.134 assume a forma:

$$
\eta(t) \simeq \eta(0) \mathrm{e}^{-i \frac{\pi}{2} \alpha^{\prime} t}
$$

Se o resíduo $\beta(s)$ tem uma forma exponencial

$$
\beta(t)=\beta(0) \mathrm{e}^{\frac{t}{2} B_{0}},
$$


a amplitude de Regge para altas energias e pequeno momento $|t|$ transferido tem a expressão:

$$
A(s, t)=\beta(0) \eta(0) \exp \left[\frac{B_{0}}{2}+\alpha^{\prime}\left(\ln s-i \frac{\pi}{2}\right)\right]\left(\frac{s}{s_{0}}\right)^{\alpha(0)}{ }_{3}
$$

\subsection{Seção de choque elástica e total.}

Usando a amplitude 3.137 se calcula a seção de choque total da contribuição de um pólo de Regge usando o teorema óptico:

$$
\sigma_{\text {total }} \underset{s \rightarrow \infty}{\sim} \frac{1}{s} \operatorname{Im} A(s, t=0) \underset{s \rightarrow \infty}{\sim} s^{\alpha(0)-1} .
$$

A seção de choque para o caso de contribuição de vários pólos é

$$
\sigma_{\text {total }}=\sim \sum_{i} A_{i} s^{\alpha_{i}(0)-1}
$$

Se vários pólos de Regge contribuem, a amplitude incluirá termos de interferências. A seção de choque diferencial é calculada de acordo à Equação 3.62 que na teoria de Regge é da forma:

$$
\frac{d \sigma}{d t}=F(t) s^{2 \alpha(t)-2}
$$

onde $F(t)$ inclui o termo de sinal e do resíduo. Substituindo una trajetória de Regge linear e a parametrização exponencial do termo do resíduo na equação 3.140 esta é expressa:

$$
\frac{d \sigma}{d t} \sim F(t) s^{2 \alpha(0)-2} \mathrm{e}^{B|t|}
$$

onde $B=B_{0}+2 \alpha^{\prime} \ln s$. O inverso desta quantidade é a largura $B^{-}$do pico que decresce com o aumento da energia. Este fenômeno é conhecido como o decremento do pico de difração que pode ser interpretado como o incremento do raio de interação $R_{\text {int }}=\sqrt{\alpha^{\prime} s}$. Este fenômeno é observado experimentalmente e não tem analogia na abordagem óptica do problema, assim o decremento do pico de difração é um resultado não trivial da teoria de Regge.

A teoria de Regge tem conseguido descrever de maneira precisa e muito simples várias colisões a altas energias como é feito por A. Donnachie e P. V. Landshoff no artigo [114] demostrando assim o poder desta abordagem fenomenológica. Neste trabalho foram ajustados dados experimentais das reações $p p, p \bar{p}, \pi^{-} p, \pi^{+} p, K^{-} p, K^{+} p$ e $\gamma p$ pela equação:

$$
\sigma_{\text {total }}=X^{\epsilon}+Y^{-\eta}
$$

\footnotetext{
${ }^{3}$ As potências de $s$ deveram ser normalizadas a um valor fixo $s_{0}$ que é o valor minimo a partir do qual a teoria de Regge poderá ser aplicada. Este valor é normalmente fixado em $s=1 \mathrm{GeV}$.
} 
como é mostrado nas figuras 3.12 .
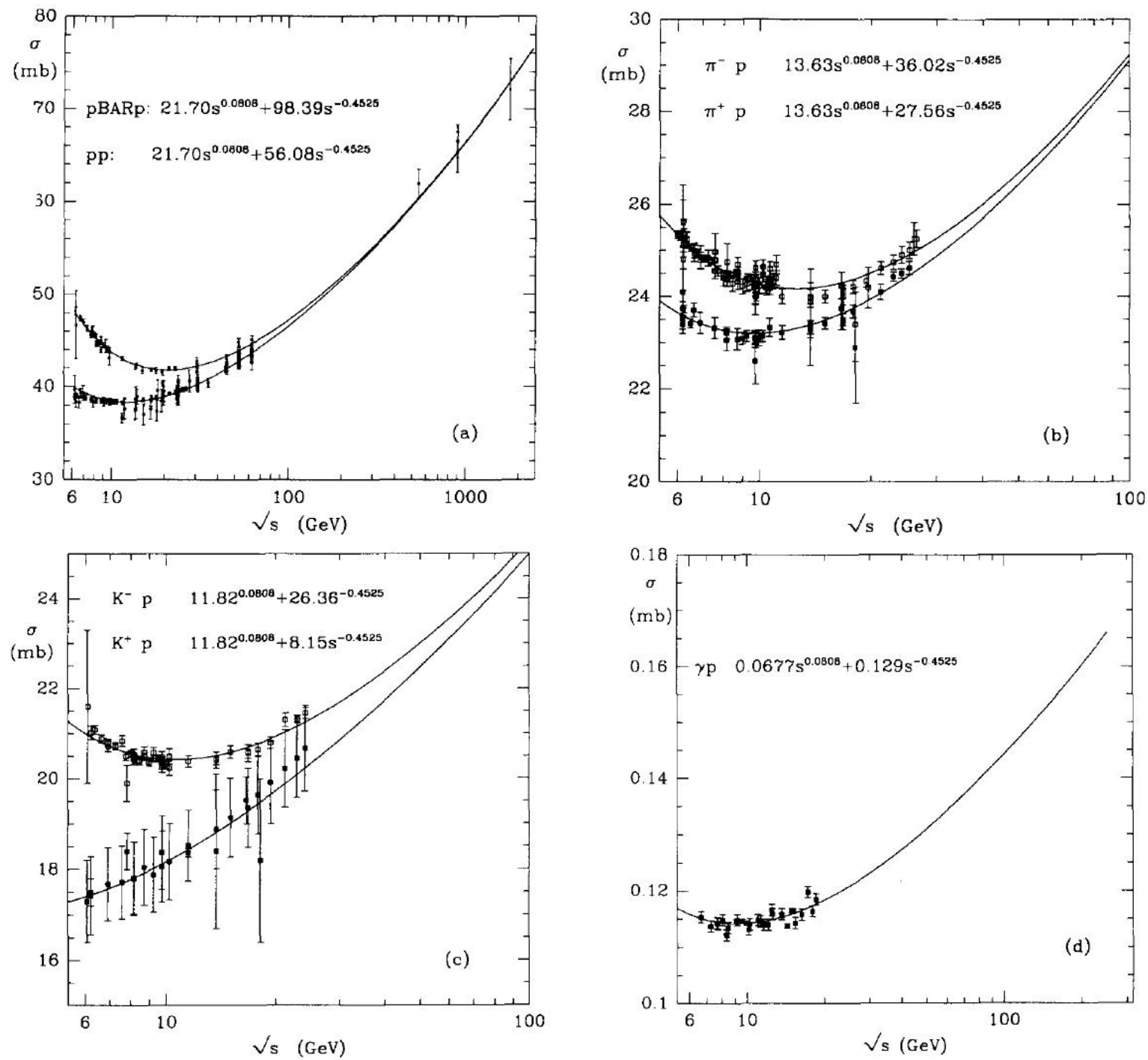

Figura 3.12: Seções de choque totais das colisões $a$ ) $p p$ e $p \bar{p}, b) \pi^{-} p$ e $\pi^{+} p$, c) $K^{-} p$ e $K^{+} p$ e $\left.d\right) \gamma p$ ajustadas usando a teoria de Regge por A. Donnachie e P. V. Landshoff. Figura publicada na Ref.[114].

Assim todas as colisões estão determinadas pela troca de um pomeron com valor de coeficiente linear $\alpha_{0 \mathbb{P}}=1.0808$ e de um reggeon com coeficiente linear $\alpha_{0 \mathbb{R}}=0.5475$ (lembrando que o expoente de $e$ é da forma $\alpha(0)-1)$. O pomeron será explicado na próxima seção.

Na prática, nos aceleradores com alvo fixo, a seção de choque total é calculada usando técnicas de transmissão i.e. pela observação da atenuação das partículas aceleradas depois que colidem com um alvo de hidrogênio líquido. Este método é muito preciso uma vez que os erros são pequenos $(0.2-0.3 \%)$. Nos colisores, a seção de choque total 
é calculada usando os números de eventos elásticos $N_{\text {elast }}$ e inelástico $N_{\text {inelast }}$ da forma:

$$
N_{\text {elast }}+N_{\text {inelast }}=\mathcal{L} \sigma_{\text {total }}
$$

onde $\mathcal{L}$ é a luminosidade do colisor. A grandeza $\mathcal{L}$ não está bem definida, então é necessário um método independente desta. Este método relaciona a seção de choque total com o espalhamento elástico para $t=0$ da forma:

$$
\frac{d N_{\text {elastica }}}{d t}=\left.\mathcal{L} \frac{d \sigma_{\text {elastica }}}{d t}\right|_{t=0}=\mathcal{L} \frac{1+\rho^{2}}{16 \pi} \sigma_{\text {total }},
$$

onde $\rho$ é a razão entre a parte real e imaginária da amplitude:

$$
\rho=\frac{\operatorname{Re} A(s, t=0)}{\operatorname{Im} A(s, t=0)} .
$$

Esta grandeza é pequena a altas energias, $\rho \sim 0.15$, então não é necessário um valor preciso dela neste intervalo de energia. Eliminando a luminosidade da equação pela substituição da equação 3.144 em 3.143 , a seção de choque total é agora:

$$
\sigma_{\text {total }}=\frac{16 \pi}{1+\rho^{2}} \frac{\left.\frac{d N_{\text {elastica }}}{d t}\right|_{t=0}}{N_{\text {elastica }}+N_{\text {inelastica }}} .
$$

Esta fórmula independente da luminosidade é a usada nos mais recentes experimentos de seção de choque total. Este método introduz erros de algumas unidades de \%, maiores que no método para alvos fixos.

\subsection{O Pomeron e o Odderon.}

Os polos de Regge relacionados com ressonâncias no canal $t$ mostradas na figura 3.11 tem valores de coeficiente linear menor que $\alpha_{0} \leq 0.5$. Este valor produz una comportamento da seção de choque mostrada na equação 3.138 que decresce com o aumento da energia $\sqrt{s}$. No entanto, experimentalmente alguns processos de espalhamentos são descritos por uma seção de choque constante no intervalo $10 \mathrm{GeV} \leq \sqrt{s} \leq 20 \mathrm{GeV}$ e que cresce para maiores energias. Para resolver esta questão foi proposta a existência de um reggeon com $\alpha_{0_{\mathbb{P}}} \sim 1[116,117]$ que foi chamado pomeron devido Ao trabalho desenvolvido por I. Ya. Pomeranchuk. A trajetória do pomeron no esta associada a nenhuma partícula conhecida. O comportamento desta trajetória indica que está associada a um glueball que resulta de uma troca de ao menos dois gluons como é mostrado na figura 3.13 na referência [118]. O pomeron é a trajetória dominante no espalhamento elástico e processos difrativos e estão caracterizados pela troca de números quânticos do vácuo no 


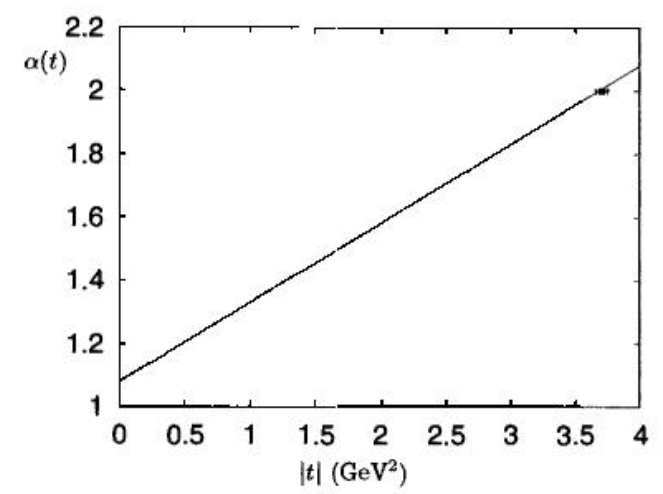

FigURA 3.13: Interpolação da trajetória do pomeron com uma partícula glueball [106].

canal $t$, assim:

$$
\mathbb{P}: \quad P=+1, \quad C=+1, \quad G=+1, \quad I=0, \quad \xi=+1 .
$$

Daí que o limite

$$
\xi \lim _{x \rightarrow 1} \frac{1+\mathrm{e}^{-i \pi x}}{\sin i x}=-i .
$$

e o termo de sinal $3.135 \eta(0)=-1$. Para $t=0$, o comportamento assintótico da amplitude é imaginário puro:

$$
A_{\mathbb{P}}(s, t=0) \underset{s \rightarrow 0}{\simeq} i \beta_{\mathbb{P}}(0) s^{\alpha_{\mathbb{P}(0)}}
$$

A seção de choque destes processos indica a trajetória do pomeron é mais plana que as trajetórias mesônicas e bariônicas, assim $\alpha_{\mathbb{P}}^{\prime} \sim 0.25 \mathrm{GeV}$ e $\alpha_{0 \mathbb{P}} \sim 1$ como foi mencionada acima. Este último valor tem a característica de saturar a seção de choque, desde que um valor do coeficiente linear maior que a unidade $\alpha_{0 \mathbb{P}}>1$ violaria o limite de FroissartMartin. Experimentalmente é encontrada incoerência ao exposto anteriormente já que a seção de choque aumenta com a energia, o que implica um valor do coeficiente linear $\alpha_{0}$ maior que a unidade. Isto pode-se abordar de várias formas. Uma delas estabelece que as energias para as quais a seção de choque cresce estão ainda longe das energias que produzem o comportamento assintótico existindo assim um mecanismo (ainda desconhecido) que produz o crescimento da seção de choque. Outra está dada pela existência de outras singularidades além de pólos no plano de momento angular complexo como são os cuts que contribuem para o crescimento da seção de choque [119-121].

O pomeron com um valor de coeficiente linear $\alpha_{0 \mathbb{P}} \sim 1.08$, como o obtido no trabalho de Donnachie e Landshoff [114], é conhecido como soft pomeron. Outros experimentos reportam valores ainda maiores e.g Covolan, Montanha e Goulianos [122] reportam 
$\alpha_{0 \mathbb{P}}=1.104 \pm 0.002$, enquanto que Cudell, Kang e Kim [123] obtêm $\alpha_{0 \mathbb{P}}=1.0964_{-0.0091}^{+0.0115}$. Os primeiros mostram ainda que usando a amplitude eikonal de Regge o valor deste coeficiente cresce ainda mais até $\alpha_{0 \mathbb{P}} \sim 1.122$. Um valor positivo do expoente da energia do centro de massa $s^{\lambda}$ leva à violação da unitariedade, porém matematicamente estes valores do coeficiente $\alpha_{0}$ não diferem de um comportamento logarítmico $\ln s$ até valores de energia que nunca poderão ser atingidos experimentalmente. Da mesma forma, a função $\ln s$ mais uma constante não difere de uma combinação de $\ln ^{2} s$. A função $\ln ^{\gamma} s$ não viola a unitariedade para valores menores do que 2 , então o valor $\alpha_{0 \mathbb{P}} \sim 1.1$, ou o que é o mesmo um valor do expoente $\sim s^{0.1}$ violará o princípio de unitariedade em energia astronomicamente gigantes. Nestas energias deverá haver um mecanismo até agora desconhecido que incluirá correções unitárias para assim modificar o comportamento da função e assim cumprir o citado princípio.

Existe um valor do coeficiente linear ainda maior $\alpha_{0 \mathbb{P}} \sim 1.15$ obtido na abordagem da cromodinâmica quântica (QCD) pertubativa conhecido como BFKL ou hard pomeron. Esta abordagem é desenvolvida por Lipatov e co-autores [124, 124-126]. O hard pomeron se comporta no plano complexo do momento angular mais como um branch cut do que como um polo. Na QCD perturbativa este pomeron é associado como uma escada (ladder) de glúons "reggenizados" interagentes. Comparar o hard pomeron com o soft pomeron é quase impossível já que que a estrutura deste último não é conhecida que é definido como "algo" que é trocado numa colisão que permite uma análise muito precisa e muito simples do processo. No entanto existem dois diferentes pontos de vista na literatura ao respeito da relação entre os dois pomerons. O primeiro é que existe só um pomeron que tem um valor do coeficiente linear que depende da virtualidade $Q^{2}$, $\alpha_{0 \mathbb{P}}\left(Q^{2}\right)$ e transita do estado soft $\left(\alpha_{0 \mathbb{P}} \sim 1.1\right)$ para valores da virtualidade $Q^{2} \sim 0$ ao estado hard $\left(\alpha_{0 \mathbb{P}} \sim 1.4\right)$ caracterizado por altos valores de $Q^{2}$. O segundo ponto de vista é que os pomerons soft e hard são objetos diferentes: o primeiro tem uma natureza não perturbativa, o segundo é um objeto perturbativo que domina o processo para altos valores de $|t|$ e $Q^{2}$, mas que é irrelevante para processo com $|t| \rightarrow 0$ e $Q^{2} \rightarrow 0$.

Outro tipo de trajetória de Regge é o odderon que é proposto como um pomeron com $C=P=-1$ por L. Lukaszuk e B. Nicolescu [127] O odderon é uma singularidade no plano $j$ da amplitude ímpar $A_{-}$no canal $t$ perto de $j=1$. Ainda não existe evidência experimental do odderon para pequenos valores de $|t|$, mas sua existência pode resolver diferenças entre as amplitudes e seções de choque do espalhamento assintótico das partículas $p p$ e $p \bar{p}$. 


\subsection{Introdução à física das colisões ultraperiféricas}

As colisões ultraperiféricas são aquelas onde os íons interagem via campo electromagnético produzido por eles. O campo electromagnético pode ser considerado como uma distribuição de fótons virtuais que interagem com o núcleo de acordo ao modelo de Dominância Vetorial [18]. Portanto, as colisões ultraperiféricas, UPCs, são reações em que dois íons interagem via nuvem de fótons virtuais. A intensidade do campo eletromagnético, ou seja, o número de fótons na nuvem em torno do núcleo, é proporcional à $Z^{2}$. Assim estes tipos de interações são altamente favorecidas quando íons pesados colidem. A figura 3.14 mostra uma visão esquemática de uma colisão ultraperiférica de íons pesados. A forma elíptica dos núcleos é devido à contração de Lorentz.

Os processos dominantes em UPCs são as interações fóton-nucleon (núcleo). As configurações atuais do detector do LHC podem explorar fenômenos hard a pequenos valores do momento transferido em núcleos e nucleons com energias do centro de massa acima de $1 \mathrm{TeV}$, ampliando assim o limite de energia para experimentos similares no HERA por um fator de dez. O LHC está na cinemática da faixa onde os efeitos não lineares são várias vezes maiores do que no HERA. Nesta faixa de energia é possível analisar as densidades inclusivas e difrativas dos pártons pela análise de vários processos nucleares. A interação de dipolos pequenos com prótons e núcleos pode ser investigada em produções elásticas e quase-elásticas dos mésons $J / \psi$ e $\Upsilon$, assim como a produção de mésons $\rho_{0}$ para grande valores de momento transferido $t$. Estritamente falando, uma in-

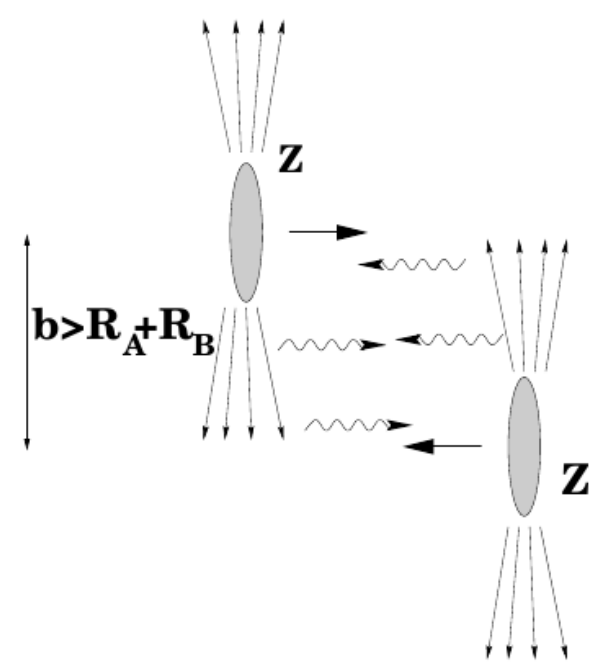

Figura 3.14: Diagrama esquemático de uma colisão ultraperiféricas entre dois íons.

teração eletromagnética ultraperiférica pode ocorrer simultaneamente com uma colisão hadrônica. No entanto, uma vez que não é possível separar as componentes hadrônicas das eletromagnéticas em tais colisões, as componentes hadrônicas são excluídas pelo 


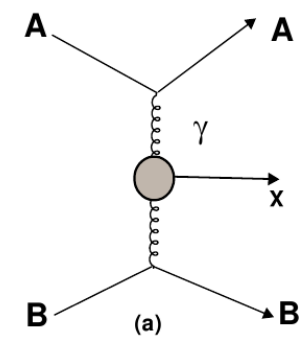

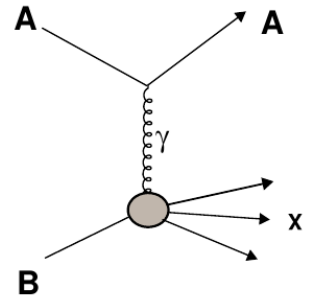

(b)

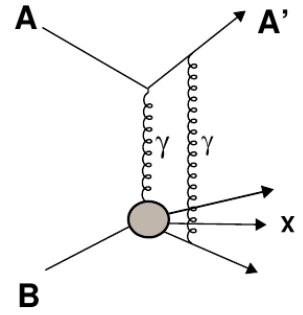

(c)

FiguRA 3.15: Diagrama esquemático: (a) da interação electromagnética onde os fótons dos íons interagem entre eles, (b) da reação fóton-núcleo onde o fóton emitido por um núcleo interage com o outro núcleo, (c) da reação fóton-resíduo nuclear onde o fóton emitido por um núcleon interage com o outro núcleo fissionado resultado de uma fotorreação com um núcleo diferente ao inicial. Figura publicada na Ref. [7]

corte no parâmetro de impacto. Assim, nos cálculos das colisões ultraperiféricas entre os núcleos $A$ e $B$, o parâmetro tem que ser maior do que a soma dos dois raios nuclear e.i. $b>R_{A}+R_{B}$.

Nas colisões ultraperiféricas existem vários tipos de reações nucleares possíveis como é mostrado nos diagramas da figura 3.15. Podem acontecer a) reações entre os fótons irradiados pelos íons, b) reações fotonucleares nos íons induzidas por fótons irradiados pelo outro íon e c) reações nucleares onde o fóton interage com um ou vários núcleos que resultam de uma possível fissão devido à uma fotorreação primaria. Algumas das características mais importantes das colisões ultraperiférica são:

- Os fótons emitidos pelos núcleos são coerentemente irradiados, fixando o limite para o valor mínimo do comprimento de onda, que tem que ser maior do que o raio do núcleo.

- O princípio de incerteza fixa o limite superior do momento transversal do fóton emitido pelo íon $A$ em $p_{T} \lesssim \hbar c / R_{A} \sim 28(330) M e V / c$ para jatos de $\mathrm{Pb}(p)$, enquanto que o momento longitudinal $p_{L}$ é modificado pelo fator de Lorentz. Por exemplo, a energia máxima de colisão para $\gamma \gamma$ numa colisão simétrica $A A$ é $2 \hbar c \gamma_{L} / R_{A}$. $\approx 6 \mathrm{GeV}$ no Relativistic Heavy Ion Collider (RHIC) e $\approx 200 \mathrm{GeV}$ no Large Hadron Collider (LHC).

- A intensidade do campo electromagnético, e com ele, o número de fótons virtuais na nuvem ao redor do núcleo atômico é proporcional à $Z^{2}$.

- Os íons irradiam fótons quase reais com virtualidade $q^{2}<\left(\hbar c / R_{A}\right)^{2}$ onde $R_{A}$ é o raio do núcleo $A$. A virtualidade pode ser desconsiderada se é menor que $(60 \mathrm{MeV})^{2}$, ou seja, para núcleos pequenos $(A>16)$. 
- Como a energia dos fótons é menor que a energia dos nucleons, as interações fotonucleares têm menor energia de centro de massa do que as interações hádron - hádron.

- Mesmo que a energia das fotorreações seja menor, a dependência do fluxo de fótons com o quadrado da carga elétrica aumenta a taxa deste tipo de reações.

- Mesmo que os fótons sejam quase reais, altas energias provocam fotorreações com alta virtualidade $Q^{2}$ no sistema de centro de massa do fóton e o párton.

- Portanto, mésons vetoriais e quarks pesados e jatos são produzidos com grande probabilidade nas colisões ultraperiféricas.

\subsection{A física das colisões ultraperiférica no LHC}

Os dados experimentais do Hadron Electron Ring Accelerator (HERA) mostram que a distribuição de glúons e o mar de quarks cresce com a diminuição da fração do momento $x$ ao ponto que, para valores suficientemente pequenos de $x$, a densidade dos pártons e prótons vai até um incremento proporcional à potências de $\ln (1 / x)$. O aumento da densidade dos pártons é regulado pelos fenômenos de "shadowing " e recombinação ex. $g g \rightarrow g$, assim como possíveis tunelamentos entre diferentes vácuos da QCD. O processo de espalhamento com os nucleons será dominante para valores pequenos de $x$. O grande valor das densidades de glúons medidas no experimento HERA demonstram a existência de efeitos não lineares no comportamento do momento transferido ao quadrado de fótons virtuais com virtualidade até $Q^{2} \sim 4 G e V^{2}$, para os valores de $x$ estudados. No LHC, estes fenômenos poderão ser vistos para maiores valores de $x$ em colisões de íons pesados.

Os experimentos realizados no HERA foram muito importantes para o entendimento da física das interações forte nas altas energias. Foi observado um rápido crescimento de pártons com pequenos valores de $x$ num amplo intervalo de $Q^{2}$, assim como uma probabilidade significativa de espalhamento com altos valores de momento transferido (hard difraction) com uma dependência logarítmica de $Q^{2}[15,16]$. Foi encontrado um novo tipo de processo hard exclusivo que é a produção de mésons vetoriais leves para grandes valores de $Q^{2}$ e a produção de mésons vetoriais pesados para qualquer valor de $Q^{2}[15,16]$ que mostra que os dipolos $q \bar{q}$ interagem com hádrons. Estudos combinados de deep inelastic scattering (DIS) e produção hard de mésons vetoriais sugerem que a força da interação alcança o maior valor possível para $Q^{2}<4 G e V^{2}$, conhecido como regime do disco escuro (BDR). Esta conclusão foi confirmada nos estudos de espalhamento hard inclusivo [128]. 
No entanto, o intervalo de $Q^{2}$ sobre a qual o BDR existe é relativamente pequeno, com valores ainda menores para processos hard onde acontecem acoplamentos de dipolos $q \bar{q}$ com $Q^{2} \sim 1 \mathrm{GeV}^{2}$, tornando difícil separar os efeitos perturbativos dos não perturbativos e assim fazer conclusões corretas. A existência de um regime de interação onde aconteçam, com uma alta probabilidade, processos com altos valores de momento transferido à pequenos valores de $x$ podem ser uma característica de interações fortes à altas energias. Isto é devido ao fato que a densidade de glúons do alvo é alta para pequenos valores da fração de momento $x$.

O começo do regime do disco escuro corresponde ao começo da dinâmica não linear da QCD, i.e um drástico término do regime linear da QCD, onde na cinemática os processos DIS e o espalhamento exclusivo podem ser tratados como uma interação de pequenos dipolos com o alvo. A seção de choque inelástica da interação de um dipolo quarkantiquark de tamanho $d$ com um hádron, usando a aproximação leading - log, é dada pela fórmula $[129,130]$

$$
\left.\sigma_{\operatorname{dip} h}\left(s_{\operatorname{dip} h}, d^{2}\right)=\frac{\pi^{2}}{4} C_{F}^{2} d^{2} \alpha_{s}\left(Q_{e f f}^{2}\right) x g\left(x, Q_{e f f}^{2}\right)\right)
$$

onde $x=Q_{e f f}^{2} / s_{\operatorname{dip} h}$ e $s_{\operatorname{dip} h}$ é a energia de centro do massa para o sistema hádrondipolo, $C_{F}^{2}$ é o operador de Casimir que é igual a $4 / 3$ para o dipolo $q \bar{q}$ e 3 para $g g$, $\alpha_{s}\left(Q_{e f f}^{2}\right)$ é a constante de acoplamento forte leading - order (LO) e $\left.g\left(x, Q_{e f f}^{2}\right)\right)$ é a densidade de glúons LO no alvo. Estas duas últimas grandezas são calculadas para $Q_{e f f}^{2} \propto d^{-2}$.

A densidade dos glúons aumenta com a diminuição de $x$, a seção de choque na equação 3.150 , pode torna-se maior do que é permitido pela restrição de unitariedade, $\sigma_{\operatorname{dip} h}\left(s_{\operatorname{dip} h}, d^{2}\right)>$ $\pi r_{h}^{2}$, onde $r_{h}$ é o raio transversal da distribuição de glúons no hádron para o valor de $x$ correspondente. A restrição de unitariedade corresponde a uma absorção total para valores de parâmetros de impacto $b \leq r_{h}$, então a seção de choque incluíra o efeito de absorção para valores pequenos de $b$. Se o regime de absorção total em $b \leq r_{h}$ é alcançado, a seção de choque de absorção torna-se aproximadamente igual à seção de choque inelástico. Em energias suficientemente altas, os campos de glúons com pequenos valores de $x$ caracterizados pela cor dos dipolos tornam-se tão fortes que o dipolo não pode se propagar através da matéria nuclear sem que aconteça absorção, sinalizando o colapso do regime linear da equação 3.150 e assim, o início do BDR.

Na aproximação de dipolo, um fóton de alta energia pode ser considerado como uma sobreposição de dipolos que interagem no BDR com energia crescente. Fótons contêm maior quantidade de dipolos pequenos do que hádrons, levando a um crescimento mais rápido da $\sigma_{\operatorname{dip} h}(\gamma p)$ que é o valor estabelecido para a seção de choque de hádrons 
restringida pelo limite de Froissart-Martin. Assim, as interações de fótons reais são sensíveis a estes pequenos dipolos. Como resultado, um grande número de questões teóricas acerca do início da BDR podem ser estudadas utilizando UPCs. O valor da energia perto do limiar do BDR onde a secção de choque de interação está determinada pela restrição de unitariedade e não apresenta um crescimento tão rápido, é pouco conhecida. Tambén não é conhecida a dependência energética. Além disso, a energia em que a seção de choque dipolo-alvo faz a transição de cor que vai desde transparente (processo soft) à opaco (processo hard) e ao BDR é uma das questões da física moderna que debe ser determinada.

Muitas questões podem ser respondidas selecionando processos onde glúons interagem diretamente. Para isso, é necessário que os experimentos sejam levados à energias mais altas e pequenos valores de $x$, assim como a maior densidade gluônica utilizando vários núcleos, para uma mesma energia e valor de $x$. Alguns experimentos foram propostos pelo HERA mas ainda não foram implementados [131]. As colisões hadrônicas $p p$ e $p A$ tem a dificuldade de apresentar um grande background devido às múltiplas colisões com altos valores de momento transferido no núcleo que impede medições com valores de virtualidade $Q^{2}<100-200 \mathrm{GeV}^{2}$. Assim, para alcançar o regime de pequenos valores da fraç̧ão de momento $x$, muitas das abordagens utilizadas no HERA poderiam ser implementadas no LHC, pelas UPCs, em colisões $A A$ e $A p$.

O principal objetivo das UPCs do tipo $p A$ e $A A$ é o estudo das interações com alto momento transferido que mostram a presença de uma alta densidade gluônica. Espalhamento com altos valores de momento transferido em alvos nucleares ampliam a faixa de pequenos valores da fração de momento $x$ em três ordens de grandeza, e assim, os pontos mais importantes dos experimentos do HERA: $i$ ) medições de altas densidades de glúons, $i i$ ) espalhamento com altos valores de momento transferido induzidos por glúons e iii) os processos exclusivos de produção dos mésons vetoriais $J / \Psi$ e $\Upsilon$ que podem ser estudados nas UPCs de colisões tipo $A B$ e $p A$. Na figura 3.16 é mostrado o intervalo de trabalho nas UPCs no LHC, o qual mostra que estas podem aprofundar a presença de glúons nos prótons e no núcleo na produção de quarkonium, dijets e hádrons. É mostrado também o valor típico de $Q^{2}$ para o processo exclusivo de fotoprodução dos mésons pesados $J / \Psi$ e $\Upsilon$. Ao mesmo tempo, na figura 3.16 são mostrados os intervalos cinemáticos para $J / \Psi$ no RHIC, os dados $F_{2}^{A}$ e $\sigma_{L}^{A}$ no eRHIC e a produção de $Z^{0}$ no LHC.

O interesse em colisões electromagnéticas de íons relativísticos em aceleradores de partículas está relacionado também com questões de importância prática. A colisão relativística de um núcleo, incluindo as interações hadrônica e electromagnética, resulta em uma mudança das propriedades deste como as emissões de núcleons, e com elas mudanças na 


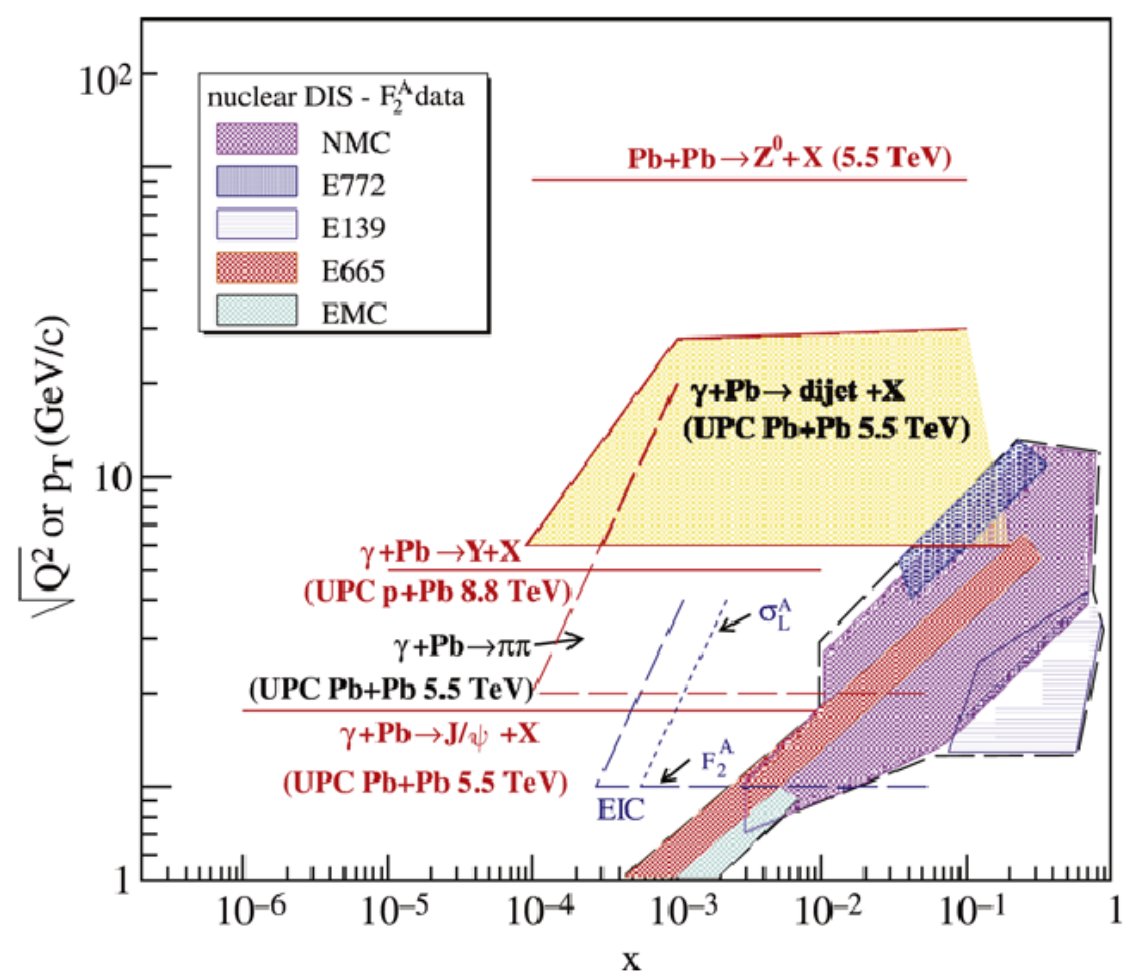

Figura 3.16: Diagrama esquemático do intervalo cinemático das colisão ultraperiféricas no LHC. Figura publicada na Ref. [7].

massa, na carga e na trajetória do núcleo. O fato de que a seção de choque da interação electromagnética excede grandemente à seção de choque da componente hadrônica da interação, é ela quem determina o tempo de vida do jato no acelerador. Outro aspecto de interesse é que devido aos fragmentos nucleares criados pelo fenômeno da dissociação electromagnética, pode causar exposição à radiação aos materiais que compõem o acelerador. Então um modelo atualizado que simule corretamente o fenômeno de dissociação electromagnética é importante também para tratar estas questões.

\subsection{Efeitos electromagnéticos em colisões de íons rela- tivísticas}

As reações fotonucleares induzidas por fótons levam a um fenômeno bem conhecido na literatura como a dissociação ou fragmentação electromagnética (EMD) em colisões de núcleos relativísticos. Este processo é a segunda fonte mais importante de produção de íons depois da interação hadrônica. Ao contrário de outros processos que acontecem neste tipo de colisões, como a produção de pares de partículas ligadas, a EMD resulta num amplo espectro de fragmentos emitidos como nêutrons, prótons, mésons e fragmentos nucleares leves. O objetivo desta sessão é abordar os principais processos que 
acontecem neste tipo de colisão e que podem ser simulados pelo código CRISP, desde que eles sejam importantes como background de reações nucleares de particular interesse nos colisores de íons pesados como o LHC.

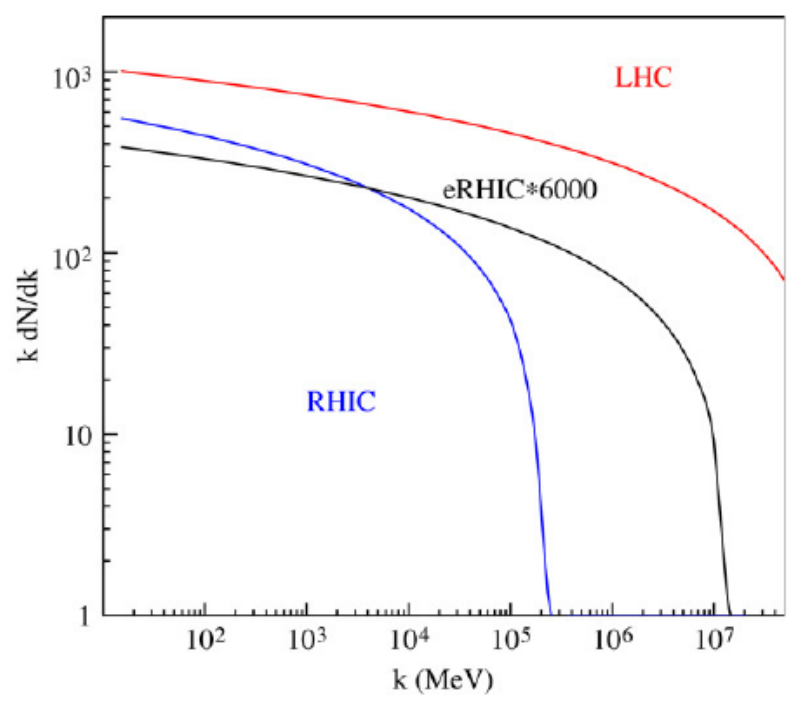

Figura 3.17: O fluxo de fótons para uma energia de centro do massa de $\sqrt{s_{N N}}=200$, $\mathrm{GeV}$ na colisão $\mathrm{Au}+\mathrm{Au}$ no RHIC e a colisão $\mathrm{Pb}+\mathrm{Pb}$ com energia do centro de massa $\sqrt{s_{N N}}=5, T e V$ no LHC comparado com a colisão $e A u$ à $10 \mathrm{GeV}+100 \mathrm{GeV}$. O gráfico do RHIV é mostrado multiplicado por 6000. Figura publicada na Ref. [7].

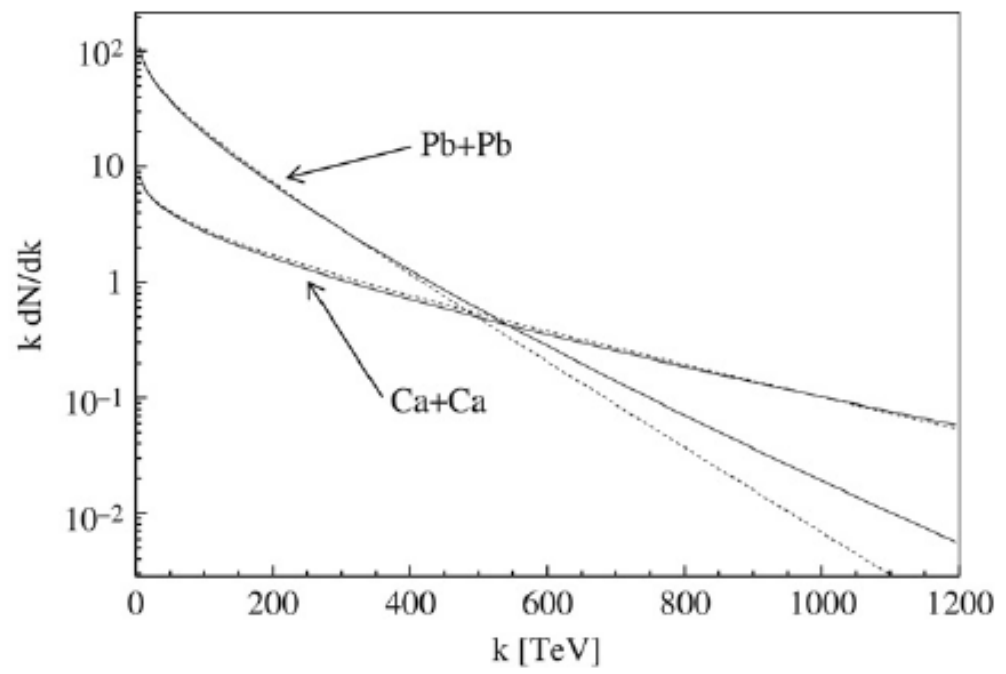

Figura 3.18: $\mathrm{O}$ espectro de fótons para colisões ultraperiféricas dos núcleos $\mathrm{Pb}+\mathrm{Pb}$ e Ca + Ca no LHC para o valor da energia de repouso do núcleo alvo. Figura publicada na Ref. [7]. 


\subsection{Produção de ressonâncias gigantes nas colisões ultra- periféricas}

$\mathrm{Na}$ excitação de um núcleo atômico induzido por colisões de íons relativísticos está incluída, num estreito intervalo de energia, a produção de ressonâncias gigantes. Estas ressonâncias usualmente decaem dentro do núcleo pela emissão de partículas ou pela fissão do núcleo para o caso de núcleos pesados como o urânio. No trabalho de C. A. Bertulani e G. Baur [132] é feita uma estimativa da contribuição multipolar da excitação de ressonâncias nos íons pesados deste processo. Nesta seção exporemos brevemente este tema.

A seção de choque de fragmentação pode ser separada em multipolos. Se, por simplicidade são usados só os termos dipolar eléctrico e quadripolar magnético, a seção de choque fica da forma:

$$
\sigma_{C F} \simeq \frac{n_{E 1}\left[E_{G R}^{(1)}\right]}{E_{G R}^{(1)}} \int \sigma_{\gamma}^{E 1}\left(E_{\gamma}\right) d E_{\gamma}+n_{E 1}\left[E_{G R}^{(2)}\right] E_{G R}^{(2)} \int \frac{\sigma_{\gamma}^{E 2}\left(E_{\gamma}\right) d E_{\gamma}}{\left(E_{\gamma}\right)^{2}}
$$

Onde os fatores $n_{E 1}(\omega)$ e $n_{E 2}(\omega)$, com boa aproximação, podem ser tirados da integral. Em núcleos pesados as ressonâncias relacionadas aos multipolos $E_{1}$ e $E_{2}$ têm picos em torno de [132]:

$$
\begin{aligned}
& E_{G R}^{(1)}=80 / A^{1 / 3} M e V, \\
& E_{G R}^{(2)}=62 / A^{1 / 3} M e V .
\end{aligned}
$$

Ao usar as regras de soma mostradas na referência [132], as seções de choque são dadas por:

$$
\begin{aligned}
& \int \sigma_{\gamma}^{E 1}\left(E_{\gamma}\right) d E_{\gamma} \simeq 60 \frac{N Z}{A} M e V \cdot m b \\
& \int \frac{\sigma_{\gamma}^{E 2}\left(E_{\gamma}\right) d E_{\gamma}}{\left(E_{\gamma}\right)^{2}} \simeq 0.22 Z A^{2 / 3} \mu b / M e V .
\end{aligned}
$$

Na figuras 3.19 e 3.20 são mostradas as contribuições dipolar magnética e dipolar e quadripolar eléctrica a seç̧ão de choque de produção de ressonâncias na colisão ultraperiférica ${ }^{40} \mathrm{Ca}+{ }^{238} \mathrm{U}$ [132]. A figura mostra que a contribuição quadripolar é muito importante nas energias médias e a dipolar é quase $90 \%$ do total nas altas energias. A excitação de ressonâncias magnéticas podem ser despreciadas desde que nas energias medianas $n_{M 1} \ll n_{E 1}$ dado por o fato $n_{M 1} \simeq\left((v / c)^{2} n_{E 1}\right)$ e nas altas energias, onde $n_{M 1} \simeq n_{E 1}$, a seção de choque de produção de ressonâncias magnéticas cumprem $\sigma^{M 1} / \sigma^{E 1} \simeq\left(\mu / e R_{t}\right)^{2}=\left(\hbar / 2 m_{N} c R_{t}\right)^{2} \ll 1$. 


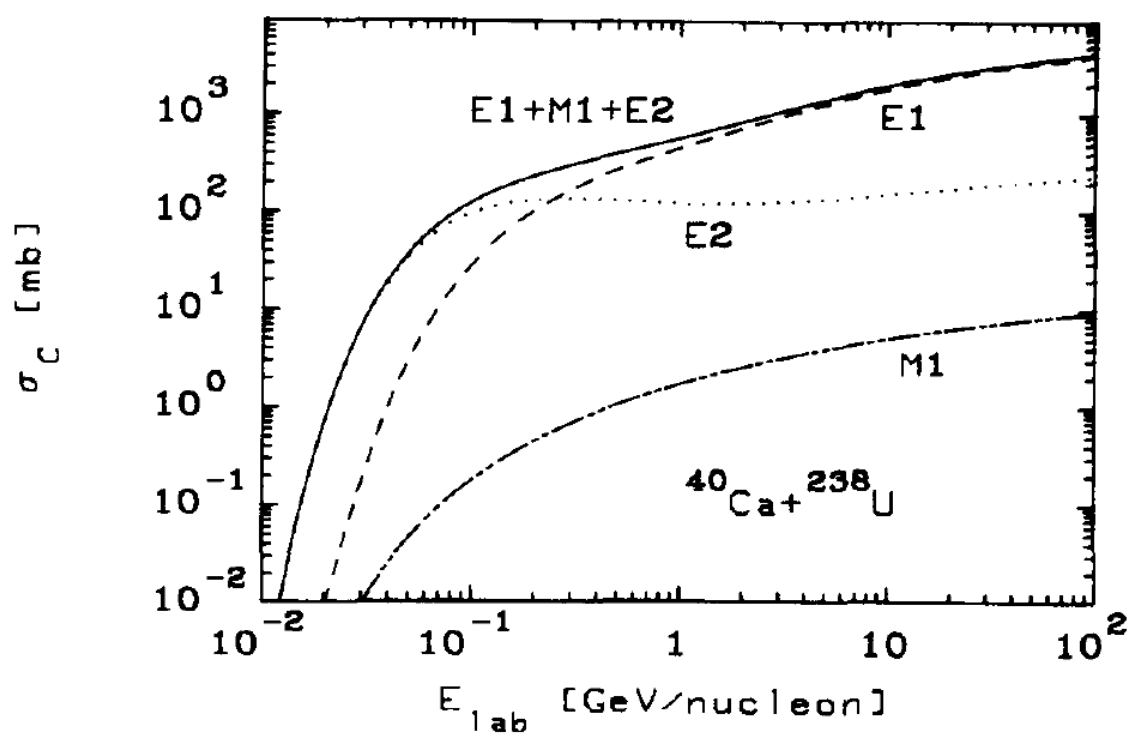

Figura 3.19: Diagrama das contribuições à seção de choque de produção de ressonâncias na colisão ultraperiférica ${ }^{40} \mathrm{Ca}+{ }^{238} \mathrm{U}$. Na figura, a linha traço-pontilhada corresponde à contribuição magnética, a linha tracejada à dipolar eléctrica, a linha pontilhada à contribuição quadripolar eléctrica e a linha cheia à soma das duas. Figura publicada na Ref. [133].

\subsection{Produção de píons nas colisões ultraperiféricas}

Um dos temas mais importantes relacionado à fragmentação electromagnética é a produção de píons. Os píons são uma fonte de informação das interações hadrônicas que acontecem no núcleo atômico. Para colisões acima do limar de produção de píons, a seção de choque é dominada por este processo e pode ser calculada da forma:

$$
\sigma_{\gamma X}=A_{e f f}(\omega)\left(\frac{Z}{A} \sigma_{\gamma, \text { próton }}(\omega)+\frac{N}{A} \sigma_{\gamma, \text { nêutron }}(\omega)\right),
$$

como mostram os experimentos e usando o efeito de sombreamento, $A_{\text {eff }}$ pode ser tomada aproximadamente independente da energia, com a dependência $A_{\text {eff }} \simeq A^{\alpha}$ onde $\alpha=0.6-0.9$. Se, por simplicidade é assumido que $\sigma_{\gamma \text {,nêutron }}=\sigma_{\gamma, \text { próton }}$, a seção de choque de produção de píons é da forma:

$$
\sigma(X Y \rightarrow \pi X Y)=\int_{140 \mathrm{MeV}}^{\infty} n_{1}\left(E_{\gamma}\right) A_{2}^{\alpha} \sigma_{\gamma p}\left(E_{\gamma}\right) \frac{d E_{\gamma}}{E_{\gamma}}+\int_{140 \mathrm{MeV}}^{\infty} n_{2}\left(E_{\gamma}\right) A_{1}^{\alpha} \sigma_{\gamma p}\left(E_{\gamma}\right) \frac{d E_{\gamma}}{E_{\gamma}}
$$

onde $n_{1}$ é o espectro de fótons equivalentes gerados pelo núcleo $X$ e $n_{2}$ corresponde ao caso contrário. O resultado da integração da equação 3.157 é mostrado na figura 3.21 


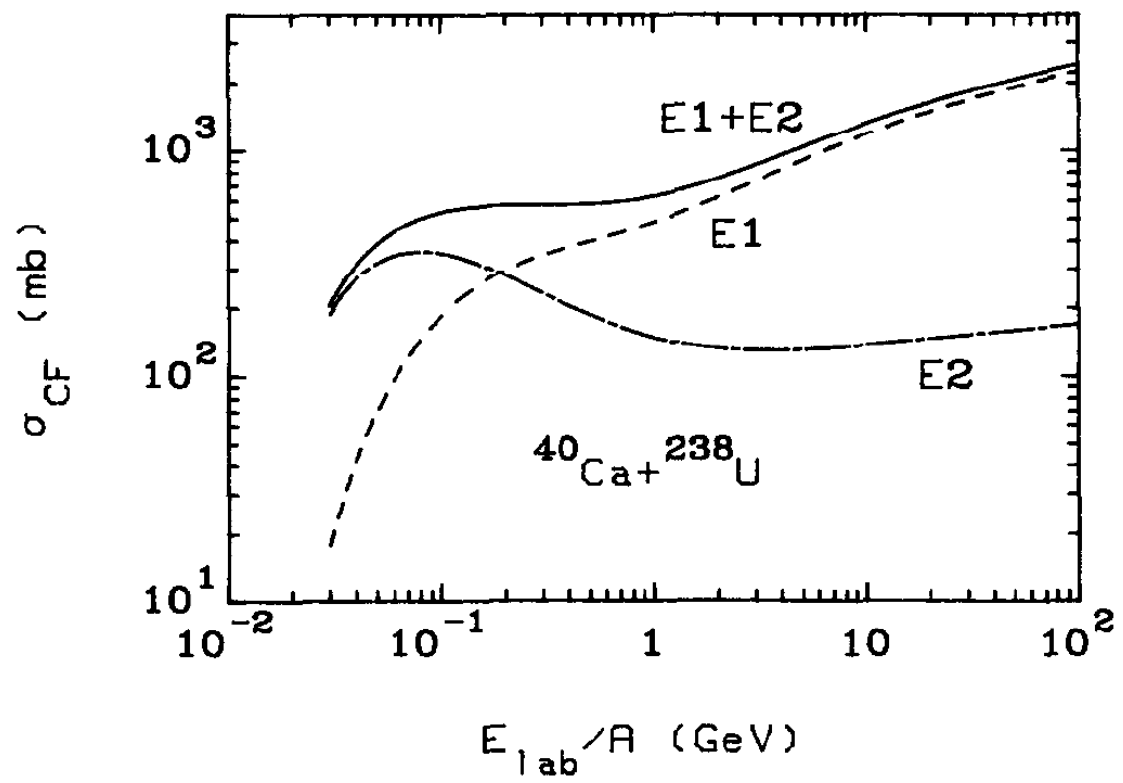

Figura 3.20: Diagrama das contribuições à seção de choque de produção de ressonâncias na colisão ultraperiférica ${ }^{40} \mathrm{Ca}+{ }^{238} \mathrm{U}$. A figura é uma ampliação da figura 3.19 que só contem a parte eléctrica da contribuição onde a linha tracejada corresponde à contribuição dipolar eléctrica, a linha traço-pontilhada à contribuição quadripolar eléctrica e a linha cheia à soma das duas. Figura publicada na Ref. [133].

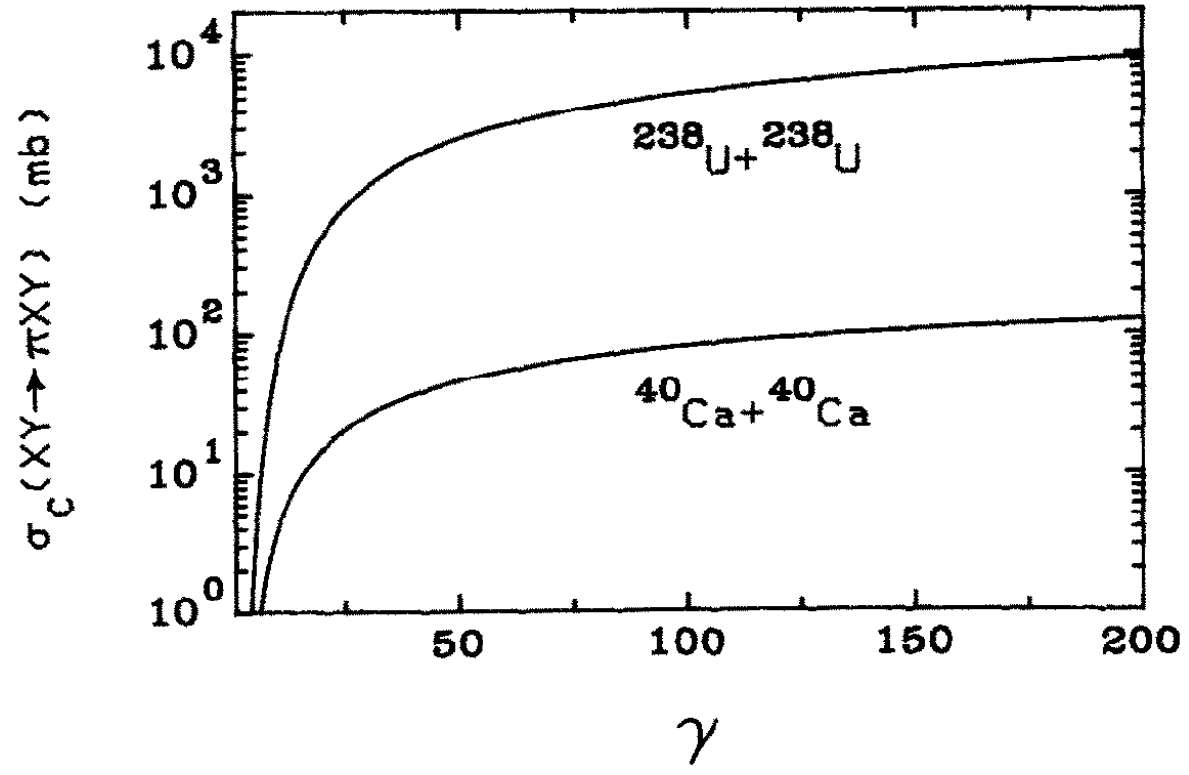

FiguRA 3.21: Diagrama da seção de choque de produção de píons para colisões ultraperiféricas dos núcleos $\mathrm{Pb}+\mathrm{Pb}$ e $\mathrm{Ca}+\mathrm{Ca}$ em função do fator de Lorentz $\gamma$.

para as reações ${ }^{238} \mathrm{U}+{ }^{238} \mathrm{U}$ e ${ }^{40} \mathrm{Ca}+{ }^{40} \mathrm{Ca}$ para vários valores do fator de Lorentz $\gamma$ que é calculado no trabalho de C. A. Bertulani e G. Baur [132]. 


\subsection{Mecanismo de interação inicial}

Como foi explicado acima na seção 3.13.1, a forma mais simples de estudar o mecanismo de reação de uma colisão nuclear ultraperiférica relativista é dada pelo método de fluxo de fótons equivalente de Weizsäcker-Williams. Este será o modelo usado em nosso trabalho como mecanismo de interação inicial na simulação de colisões ultraperiféricas no código CRISP.

A ferramenta fundamental para o estudo destas colisões é o método de WeizsäckerWilliams como foi explicado na equação 3.169. Usando uma extensão deste método para o número de fótons, pode ser feito uma análise multipolar do campo electromagnético; assim a seção de choque de excitação pode ser escrita:

$$
\sigma_{C}=\sum_{\pi l} \int n_{\pi l}(\omega) \sigma_{\gamma}^{\pi l}(\omega) \frac{d \omega}{\omega}
$$

onde $n_{\pi l}(\omega)$ é o número equivalente de fótons com energia $\hbar \omega$ para a radiação de multipolaridade de ordem $l$ eléctrica $(\pi=E)$ e magnética $(\pi=M)$ e $\sigma_{\gamma}^{\pi l}(\omega)$ é a seção de choque de fotoabsorção para cada valor de $l$ e $\pi$. A expressão analítica de todos os termos são mostrados na referência [134]. Para os primeiros termos da expansão:

$$
\begin{aligned}
& n_{E 1}(\omega)=Z^{2} \alpha \frac{2}{\pi}\left(\frac{c}{v}\right)^{2}\left[\xi K_{0} K_{1}-\frac{v^{2} \xi^{2}}{2 c^{2}}\left(K_{1}^{2}-K_{0}^{2}\right)\right] \\
& n_{E 2}(\omega)=Z^{2} \alpha \frac{2}{\pi}\left(\frac{c}{v}\right)^{4}\left[2\left(1-\frac{v^{2}}{c^{2}}\right) K_{1}^{2}+\xi\left(2-\frac{v^{2}}{c^{2}}\right)^{2} K_{0} K_{1}-\frac{\xi^{2}}{2}\left(\frac{v}{c}\right)^{4}\left(K_{1}^{2}-K_{0}^{2}\right)\right] \\
& n_{M 1}(\omega)=Z^{2} \alpha \frac{2}{\pi}\left[\xi K_{0} K_{1}-\frac{\xi^{2}}{2}\left(K_{1}^{2}-K_{0}^{2}\right)\right]
\end{aligned}
$$

onde $\mathrm{Z}$ é o número atômico, $\alpha$ é contante da estrutura fina, $K_{n}$ são as funções de Bessel modificadas de ondem $n, \gamma$ é o fator de Lorentz e $\xi$ é o parâmetro adiabático $\xi=\omega R / \gamma v$, onde $R$ é o valor mínimo do parâmetro de impacto. Para baixos valores do fator $\gamma$, que não é nosso casso, é importante levar em conta o recuo do núcleo e para isso, a referência [135] sugere a aproximação $\xi=(\omega / \gamma v)\left(R+\pi Z_{1} Z_{2} e^{2} / 2 m_{0} v^{2} \gamma\right)$, onde $m_{0}$ é a massa reduzida dos dois núcleos.

A separação da sesão de choque da forma 3.158 é útil nas reações que têm uma multipolaridade favorecida e para calcular a contribuição de cada multipolaridade no processo.

A probabilidade de que aconteça um processo electromagnético numa colisão nuclear ultrarelativística é dada pela fórmula:

$$
P(b)=\int I(\omega, b) \sigma_{\gamma}(\hbar \omega) d(\hbar \omega)=\int N(\omega, b) \sigma_{\gamma}(\omega) \frac{d \omega}{\omega}
$$


onde $I(\omega, b)$ é a intensidade do campo electromagnético por unidade de energia, por unidade de parâmetro de impacto, por unidade de área, $\sigma_{\gamma}(\hbar \omega)$ é a seção de choque fotonuclear com energia $E_{\gamma}=\hbar \omega$. A grandeza $N(\omega, b)$ pode ser interpretada como o número de fótons equivalentes por unidade de área, por unidade de energia e parâmetro de impacto emitidos pelo projéctil de carga elétrica $Z$, e é da forma:

$$
N(\omega, b)=\frac{Z^{2} \alpha}{\pi^{2}}\left(\frac{\omega}{\gamma v}\right)^{2}\left(\frac{c}{v}\right)^{2}\left[K_{1}^{2}(x)+\frac{1}{\gamma^{2}} K_{0}^{2}(x)\right]
$$

onde $x=\omega b / \gamma v, \alpha=e^{2} / \hbar c$ é a constante de estrutura fina, $K_{0}$ e $K_{1}$ são as funções modificadas de Bessel de ordem 0 e 1 respetivamente. Os termos que contêm o fator relativista de Lorentz $\gamma$ estão associados ao pulso electromagnético longitudinal que é afetado pela contração relativística de Lorentz.

O fluxo de fótons por unidade de área está dado pela integral da equação 3.163 no intervalo de parâmetro de impacto que vai desde o valor mínimo $b=R$, que depende do tipo da colisão, até $b=\infty$. Nosso estudo leva em conta a contribuição de cada termo da expresão 3.158 pela inclusão direta das seções de choque de fotoprodução das ressonâncias expostas na seção 2.1.1.5 assim não é preciso de uma expansão multipolar de seção de choque, assim:

$$
\sigma=\int_{R}^{\infty} 2 \pi b P(b) d b=\int n(\omega) \sigma_{\gamma}(\omega) \frac{d \omega}{\omega}
$$

onde

$$
n(\omega)=\int_{R}^{\infty} 2 \pi b N(\omega, b) d b=\frac{2}{\pi} Z_{1}^{2} \alpha\left(\frac{c}{v}\right)^{2}\left[\xi K_{0}(\xi) K_{1}(\xi)-\frac{v^{2} \xi^{2}}{2 c^{2}}\left(K_{1}^{2}(\xi)-K_{0}^{2}(\xi)\right)\right] .
$$

onde foi introduzido o parâmetro adiabático $\xi=\omega R / \gamma v$. Se este valor de grandeza é multiplicado pela área do alvo fornece o número de fótons que interagem com o alvo, por exemplo, a distribuição do número de fótons que atingirão o alvo numa colisão ultraperiférica do tipo $A A$ é mostrado na figura 3.22 .

O código CRISP, permite estudar reações nucleares, como são as colisões ultraperiféricas, onde acontecem múltiplas processos de fotoabsorção em um núcleo atômico, criando assim varias partículas ao mesmo tempo de acordo ao canais de reação, como dêuterons, ressonâncias, píons e mésons vectoriais. Devido a natureza aleatória do código, para um mesmo valor dos parâmetros que caracterizam estas reações, como são a energia do centro de massa e o parâmetro de impacto para o as colisões ultraperiféricas, serão produzidas diferentes partículas, com diferentes de momento, ângulo de espalhamento, etc. 


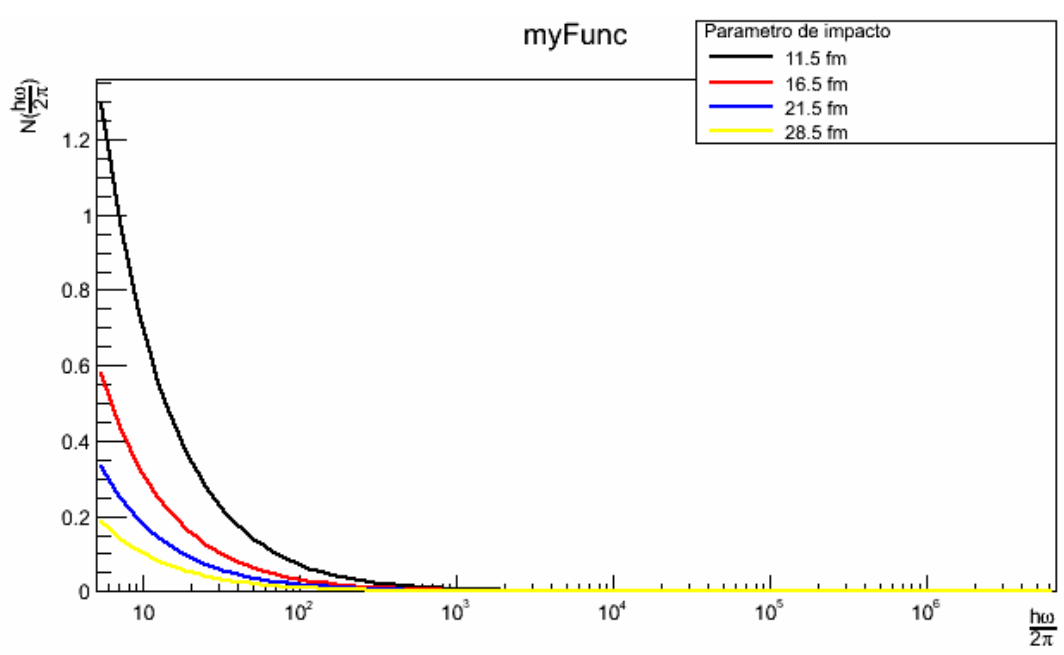

Figura 3.22: Distribução de número de fotões em função da energia numa colição ultraperiférica de tipo $A A$ para diferentes valores de parâmetro de impacto mínimo obtida com o código CRISP.

Assim o mecanismo inicial a ser usado será a simulação de varias fotorreações com uma distribuição de fótons como a mostrada na figura 3.22 que será calculada de acordo com os parâmetros da colisão a ser estudada. Estas fotorreações já estudadas de forma singular no CRISP, e que de acordo ao intervalo de energia, produzirão partículas associadas canais de reação ao longo do todo o intervalo de energias, criando assim a configuração excitada inicial que disparará a cascata intranuclear.

\subsubsection{Seção de choque}

A seção de choque para um processo de dois fótons é:

$$
\sigma_{X}=\int d k_{1} d k_{2} \frac{d L_{\gamma \gamma}}{d k_{1} d k_{2}} \sigma_{X}^{\gamma \gamma}\left(k_{1}, k_{2}\right)
$$

onde $\sigma_{X}^{\gamma \gamma}\left(k_{1}, k_{2}\right)$ é a seção de choque de produção de dois fótons do estado final e $d L_{\gamma \gamma} / d k_{1} d k_{2}$ é a luminosidade de dois fótons:

$$
\frac{d L_{\gamma \gamma}}{d k_{1} d k_{2}}=\int_{b>R_{A}} \int_{r>R_{A}} d^{2} b d^{2} r \frac{d^{3} N_{\gamma}}{d k_{1} d^{2} b} \frac{d^{3} N_{\gamma}}{d k_{2} d^{2} r}
$$

onde $d^{3} N_{\gamma} / d k_{2} d^{2} r$ é o fluxo de fótons na distância $r$ produzidos pelo núcleo de carga Z. A luminosidade pode ser multiplicada pela luminosidade íon íon resultando em uma luminosidade efetiva $d L_{\gamma \gamma}^{e f f} / d W_{\gamma \gamma}$, onde $W_{\gamma \gamma}=\sqrt{s}$ é a energia de centro de massa. Na figura 3.23 é mostrada a luminosidade efetiva para colisões de vários íons publicada na referência [7]. 

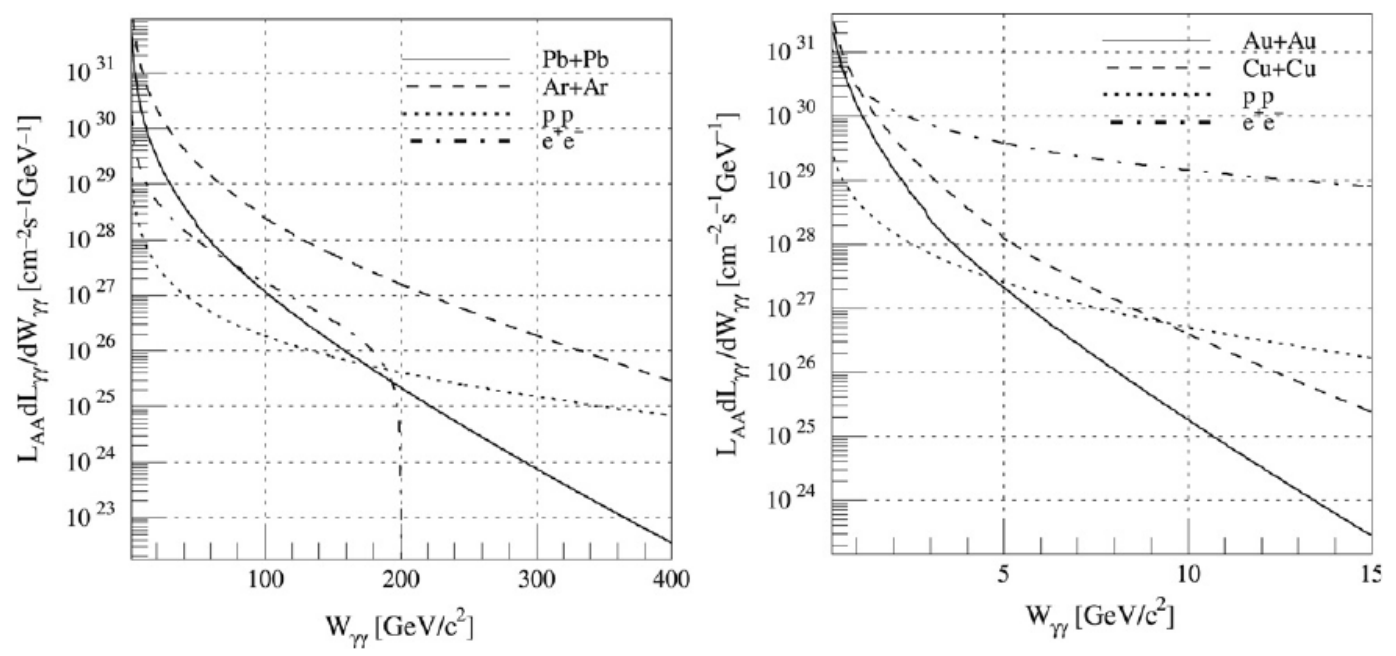

Figura 3.23: Luminosidade efetiva $\gamma \gamma$ no LHC (direita) e no RHIC para diferentes colisões de íons. Figura publicada na Ref. [7]

A seção transversal de fotoprodução também pode ser fatorada no produto da seção transversal fotonuclear e o fluxo de fótons $d N_{\gamma} / d k$ :

$$
\sigma_{X}=\int d k \frac{d N_{\gamma}}{d k} \sigma_{X}^{\gamma}(k)
$$

onde $\sigma_{X}^{\gamma}(k)$ é a seção de choque fotonuclear. O fluxo de fótons usado para calcular a luminosidade de dois fótons na Eq. 3.167 e a seção de fotoprodução transversal na Eq. 3.168 é calculada usando o método de fluxo de fótons equivalente, que foi originalmente proposto por Fermi [136] e mais tarde desenvolvido por Weizsäcker [137] e Williams [138], conhecido na literatura como o método de Weizsäcker-Williams e explicado em detalhe no livro "Classical Electrodynamics" do autor "J.D. Jackson" [139]. Este método substitui o campo gerado pelo íon projétil, que interage com o íon alvo, por uma distribuição de fótons reais cujo fluxo é uma função no espaço do parâmetro de impacto na distancia $r$ e é dado por:

$$
\frac{d^{3} N_{\gamma}}{d k d^{2} r}=\frac{Z^{2} \alpha w^{2}}{\pi^{2} k r^{2}}\left[K_{1}^{2}(w)+\frac{1}{\gamma_{L}^{2}} K_{0}^{2}(w)\right]
$$

O fluxo total que interage com o núcleo é a integral da equação 3.169 em relação a área geométrica do alvo para todos os valores do parâmetro de impacto usando sua condição de corte $b>R_{A}+R_{B}$ que não permite a componente hadrônica na colisão. Nas figuras 3.17 e 3.18 são mostrados exemplos de fluxo de colisões ultraperiféricas. Nessas colisões, o íon é acelerado cercado por uma nuvem de fótons quase reais de virtualidade $\left|Q^{2}\right|<\left(\hbar c / R_{A}\right)^{2}$, onde $R_{A}$ é o raio nuclear. Estes fótons são quase reais no sistema de medição de laboratório, mas no sistema de medição do centro de massa, devido às altas energias das colisões, as interações acontecerão com fótons de alta virtualidade. 


\section{Capítulo 4}

\section{Fótoprodução dos mésons vetoriais no ambiente nuclear.}

Neste capítulo são descritos os modelos usados nos processos de fotoprodução e interação no estado final dos mésons vetoriais no núcleo que foram incluídos no código CRISP.

\subsection{Fotoprodução de mésons vetoriais pelo modelo de soft dipole Pomeron}

Os resultados experimentais sobre a fotoprodução de mésons vetoriais mostram que estes processos podem ser naturalmente explicados utilizando o modelo de Pomeron dependente da massa. A teoria de Regge [140] tem sido bem sucedida em descrever interações soft hádron-hádron. A fotoprodução elástica de $\rho_{0}$ e mésons $\phi$ é bem descrita $[108,141,142]$ pela abordagem de um processo de espalhamento usando o modelo de dominância vetorial (VDM) [18] e a teoria de Regge [140]. Este processo de espalhamento soft pode ser descrito pela troca de uma trajetória de Regge de um soft Pomeron $\alpha(t)=$ $\alpha(0)+\alpha^{\prime} t$, com o valores de coeficientes linear $\alpha(0)=1.08$ e angular $\alpha^{\prime}=0.25 \mathrm{GeV}^{-2}$, conhecido como Pomeron não perturbado. No entanto, o comportamento do processo de fótoprodução elástico do méson $J / \Psi$ no HERA, não pode ser descrito usando o modelo do Pomeron não perturbado.

O modelo de Haakman, Kaidalov e Koch [143] descreve a fotoprodução dos mésons vetoriais, além da dependência da seção de choque com a virtualidade $Q^{2}$ do méson $\rho_{0}$. O modelo é baseado em um pomeron com interseção $\sim 1.02$, cujo comportamento é modificado à forma soft pela abordagem de multi-troca de pomerons nas interações hádron-hádron. Usando o modelo de extensão eikonal de Regge off shell, Petrov e 
Produking [144] conseguem a correta descrição da seção de choque da produção dos méson $\rho_{0}$ e $J / \Psi$. Para ele, foi proposto um pomeron universal com um valor da interseção $\sim 1.1$ obtido da descrição dos processos $p p$ e $\bar{p} p$. O modelo usa uma trajetória de pomeron independente de $Q^{2}$, leva em conta correções unitárias e não viola a unitariedade para os processos hádron-hádron e a produção de mésons vetoriais.

Um modelo universal de polo de Regge foi mais tarde proposto por L. L. Jenkovszky, E. S. Martynov e F. Paccanoni para a fotoprodução de mésons vetoriais [145]. Este modelo, que usa um pomeron com interseção igual a 1, descreve a produção dos mésons $\rho_{0}, \omega, \phi$ e $J / \Psi$. O modelo do dipolo de Pomeron foi usado em [146] para os mésons pesados. Vários cálculos que usam a teoria de perturbações no QCD foram feitos [147150], mostrando que existe uma mistura dos regimes hard e soft que consegue explicar o processo.

Para a simulação da fotoprodução de mésons vetoriais neste trabalho foi escolhido o modelo do dipolo de Pomeron desenvolvído por E. Martynov, E. Predazzi e A. Prokudin [151, 152]. Este modelo foi proposto pelo autores baseado nas medições obtidas da reação de foto-produção exclusiva de méson $J / \Psi$ pelo experimento ZEUS que permitiu fazer previsões universais do processo de foto-produção de mésons vetoriais. O ponto chave do análise dos dados obtidos é o encolhimento no cone de difração observado na fotoprodução do méson $J / \Psi$, que levou a considerá-lo como um processo QCD de baixo valor de momento transferido (soft) ao invés de um processo QCD puro (hard). Isto faz possível a aplicação do modelo de troca de dipolo um soft pomeron. O modelo tem a característica de poder ser aplicado em um grande intervalo de energia e ser aplicado para todos os mésons vetoriais (universalidade). As estruturas das singularidades da amplitude no plano $J$ são mantidas, mas uma trajetória não linear é assumida. As trajetórias dos Reggeons secundários são assumidos no caso hadrônico puro por simplicidade. O modelo descreve corretamente a produção dos mésons $\rho_{0}, \omega, \phi$ e $J / \Psi$ e prediz o comportamento da seção de choque de produção do méson $\Upsilon$ para valores de energia do centro de massa desde o limiar de produção até $W \sim 250 \mathrm{GeV}$, valores de virtualidade $0 \leq Q^{2} \leq 35 \mathrm{GeV}^{2}$ e momento transferido $0 \leq|t| \leq 1.6 \mathrm{GeV}$.

O modelo apresenta o fóton como um par quark-antiquark cujo tempo de vida é dado pelo principio da incerteza e cresce com a energia do feixe $\nu$ tipo $\left.2 \nu /\left(Q^{2}+M_{V}^{2}\right)\right)$. Este tempo é bastante longo como para que o próton interage com este par quark-antiquark pela troca de um Pomeron ou Reggeon secundário. Após a interação, este par forma um méson vetorial. A ideia é que esta interação deve ser semelhante teoricamente à interação entre hádrons onde, de acordo ao princípio da teoria de Regge, o Pomeron é universal. Se a troca de um pomeron é possível, este irá ter as mesmas propriedades que nas interações hâdron-hâdron como é o tipo de singularidade, a sua posição no plano 
complexo do momento angular, a trajetória etc. O modelo também descreve a produção de mésons induzidos por fótons virtuais $\left(Q^{2} \neq 0\right)$. Enquanto, no modelo, um fóton real pode ser considerado um hádron, para um fóton virtual pode ser assumido que nenhuma nova singularidade irá aparecer e se assim forem a sua contribuição iria ser nula [153]. Isto é demostrado a partir de análises de dados experimentais [154]. O diagrama básico da interação é mostrado na fig 4.1. Em altas energias, a amplitude de espalhamento é

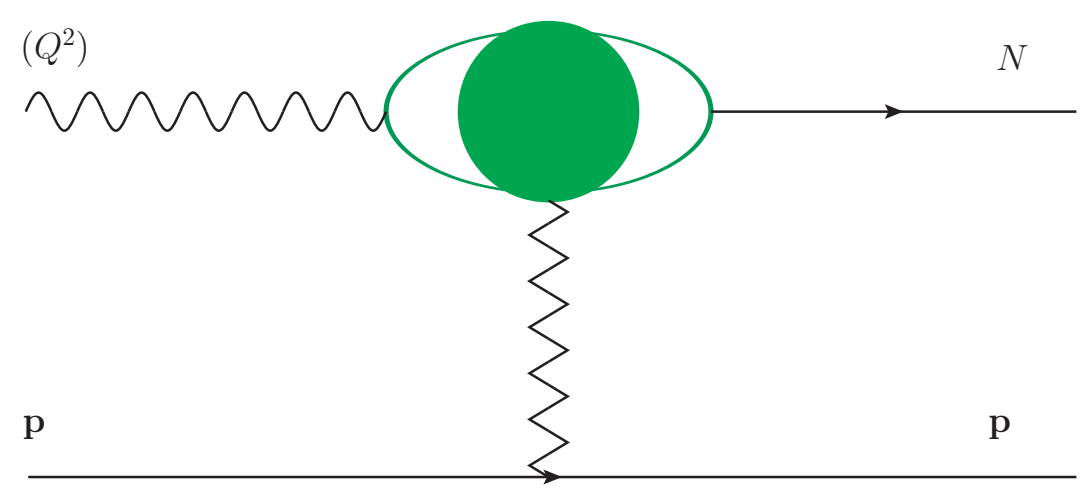

Figura 4.1: Diagrama esquemático da fotoprodução de um méson vectorial.

expressa como a soma de polos de Regge no plano complexo

$$
A(s, t)_{s \rightarrow \infty}=\sum_{i} \eta_{i}(t) \beta_{i}(t)\left(\cos \theta_{t}\right)^{\alpha_{i}(t)}
$$

onde $\eta_{i}(t)$ é o fator de forma e $\theta_{t}$ é o angulo no canal $t$. De acordo ao VDM a relação entre as seções de choque $\gamma p \rightarrow V p$ e $V p \rightarrow V p$ é dada por

$$
\frac{d \sigma}{d t}(t=0)_{\gamma p \rightarrow V p}=\frac{4 \pi \alpha}{f_{V}^{2}} \frac{d \sigma}{d t}(t=0)_{V p \rightarrow V p}
$$

onde a constante de acoplamento $4 \pi / f_{V}^{2}$ pode ser calculado a partir do processo decaimento do méson no par elétron-pósitron. No caso dos mésons $\rho_{0}, \omega, \phi, J / \Psi$ as relações desses acoplamentos são obtidos assumindo simetria de sabor SU(4). Aplicando propriedades do decaimento dos mésons vetoriais são obtidas as relações [152]

$$
m_{\rho} / f_{\rho}^{2} m_{\omega} / f_{\omega}^{2} m_{\phi} / f_{\phi}^{2} m_{J / \Psi} / f_{J / \Psi}^{2}=9: 1: 2: 8 .
$$

Estas relações são usadas pela introdução dos coeficientes $N_{V}$

$$
N_{C}=3, \quad N_{\rho}=\frac{1}{\sqrt{2}}, \quad N_{\omega}=\frac{1}{3 \sqrt{2}}, \quad N_{\phi}=\frac{1}{3}, \quad N_{J / \Psi}=\frac{2}{3},
$$

resultando na amplitude como

$$
A_{\gamma p \rightarrow V p}=N_{C} N_{V} A_{V p \rightarrow V p} .
$$


A amplitude do processo $V p \rightarrow V p$ pode ser escrita como

$$
A\left(z, t, M_{V}^{2}, \tilde{Q}^{2}\right)=\mathbb{P}\left(z, t, M_{V}^{2}, \tilde{Q}^{2}\right)+\mathbb{R}\left(z, t, M_{V}^{2}, \tilde{Q}^{2}\right)+\ldots
$$

onde $\tilde{Q}^{2}=M_{V}^{2}+Q^{2} \cdot \mathbb{P}\left(z, t, M_{V}^{2}, \tilde{Q}^{2}\right)$ é a contribuição de um pomeron e é dada por

$$
\mathbb{P}\left(z, t, M_{V}^{2}, \tilde{Q}^{2}\right)=i g_{0}\left(t, M_{V}^{2}, \tilde{Q}^{2}\right)(-i z)^{\alpha_{\mathbb{P}}(t)-1}+i g_{1}\left(t, M_{V}^{2}, \tilde{Q}^{2}\right) \ln (-i z)(-i z)^{\alpha_{\mathbb{P}}(t)-1} .
$$

O primeiro termo da soma é a contribuição de um único pólo $j$, e o segundo é a contribuição de um polo $j$ duplo. A contribuição do termo de regge é dada por

$$
\mathbb{R}\left(z, t, M_{V}^{2}, \tilde{Q}^{2}\right)=i g_{f}\left(t, M_{V}^{2}, \tilde{Q}^{2}\right)(-i z)^{\alpha_{\mathbb{R}}(t)-1} .
$$

No caso dos mésons $\rho$ e $\omega$, a amplitude de espalhamento é expressada como a soma de um Pomeron e uma contribuição de reggeon tipo $f$. De acordo com a regra de OkuboZweig-Iizuka [155-158], esta última contribuição deve ser suprimida na produção dos mésons $\phi$ e $J / \Psi$, mas devido as previsões das abordagens atuais, a contribuição reggeon tipo $f$ é adicionada para o méson $\phi$. Para o méson $\omega$ é agregada a contribuição reggeon tipo $\pi$ que é importante para uma correta descrição do espectro na energias acima do limiar. A seção de choque total elástica é dada pela expressão

$$
\sigma\left(z, M_{V}^{2}, \tilde{Q}^{2}\right)_{e l}^{\gamma p \rightarrow V p}=4 \pi \int_{t_{-}}^{t_{+}} d t\left|A\left(z, t, M_{V}^{2}, \tilde{Q}^{2}\right)\right|^{2}
$$

onde $t_{+}$e $t_{-}$são os momentos da reação do canal $t$ para o ângulo de espalhamento 0 e $\pi$ respectivamente. No caso da contribuição do Pomeron é usada uma trajetória não linear utilizada para amplitudes de fotoprodução

$$
\alpha_{\mathbb{P}}(t)=1+\gamma\left(\sqrt{4 m_{\pi}^{2}}-\sqrt{4 m_{\pi}^{2}-t}\right)
$$

onde $m_{\pi}$ é a massa do méson $\pi$. No caso da trajetória para a contribuição $f$ é escolhida a trajetória linear do Reggeon padrão

$$
\alpha_{\mathbb{R}}(t)=\alpha_{R R}(0)+\alpha_{\mathbb{R}}^{\prime}(0) t
$$

A seguinte parametrização é usada para o resíduo do Pomeron

$$
g_{i}\left(t ; M_{V}^{2}, \tilde{Q}^{2}\right)=\frac{g_{i}}{\tilde{Q}^{2}+\tilde{Q}_{i}^{2}} \exp \left[b_{i}\left(t, \tilde{Q}^{2}\right)\right]
$$


com $\mathrm{i}=0,1$. Os expoentes são parametrizados da forma

$$
b_{i}\left(t ; \tilde{Q}^{2}\right)=\left(b_{i 0}+\frac{b_{i 1}}{1+\tilde{Q}^{2} / \tilde{Q}_{b}^{2}}\right)\left(\sqrt{4 m_{\pi}^{2}}-\sqrt{4 m_{\pi}^{2}-t}\right)
$$

$\operatorname{com} \mathrm{i}=0,1$.

Para o Reggeon

$$
g_{\mathbb{R}}\left(t ; M_{V}^{2}, \tilde{Q}^{2}\right)=\frac{g_{\mathbb{R}} M_{p}^{2}}{\tilde{Q}^{2}+Q_{\mathbb{R}}^{2}} \exp b_{\mathbb{R}}\left(t ; \tilde{Q}^{2}\right)
$$

onde

$$
b_{\mathbb{R}}\left(t ; \tilde{Q}^{2}\right)=\frac{b_{\mathbb{R}}}{1+\tilde{Q}^{2} / \tilde{Q}_{b}^{2}} t
$$

A parametrização é realizada ajustando os parâmetros $g_{0}, g_{1}, Q_{0}^{2}\left(G e V^{2}\right), Q_{1}^{2}\left(G e V^{2}\right)$, $Q_{\mathbb{R}}^{2}\left(G e V^{2}\right), Q_{b}^{2}\left(G e V^{2}\right), \quad b_{00}\left(G e V^{-1}\right), \quad b_{01}\left(G e V^{-1}\right), \quad b_{10}\left(G e V^{-1}\right), b_{11}\left(G e V^{-1}\right)$, $b_{\mathbb{R}}\left(G e V^{-2}\right)$, onde $\mathbb{R}=f$ para $\rho$ e $\phi, \mathbb{R}=f, \pi$ para $\omega$. Os valores obtidos dos parâmetros pelo ajuste do modelo aos dados experimentais [152] de são mostrados na tabela 4.1.

\begin{tabular}{|c|c|c|c|c|c|c|c|}
\hline $\mathrm{N}$ & Parâmetro & Valor & Error & & Trajetória & $\alpha(0)$ & $\alpha(0)\left(G e V^{2}\right)$ \\
\hline 1 & $\gamma\left(G e V^{-1}\right)$ & $0.53853 \times 10^{-1}$ & $0.15666 \times 10^{-1}$ & & & fixo & fixo \\
\hline 2 & $g_{1}$ & $0.10435 \times 10^{-1}$ & $0.17851 \times 10^{-1}$ & 1 & $f$ Reggeon & 0.8 & 0.85 \\
\hline 3 & $g_{0}$ & $-0.32901 \times 10^{-1}$ & $0.49449 \times 10^{-4}$ & 2 & $\pi$ Reggeon & 0.0 & 0.85 \\
\hline 4 & $g_{f}$ & $0.83371 \times 10^{-1}$ & $0.49503 \times 10^{-3}$ & & Meson & No. pontos & $\chi^{2}$ per ponto \\
\hline 5 & $g_{\pi}$ & 0.60011 & $0.21962 \times 10^{-1}$ & 1 & $\rho_{0}(770)$ & $\sigma_{e l}, 127$ & 1.49 \\
\hline 6 & $Q_{0}^{2}\left(\mathrm{GeV}^{2}\right)$ & 0.0 & fixo & & $\rho_{0}(770)$ & $d \sigma_{e l} / d t, 24$ & 0.99 \\
\hline 7 & $Q_{1}^{2}\left(\mathrm{GeV}^{2}\right)$ & 0.41908 & $0.23586 \times 10^{-2}$ & 2 & $\omega(782)$ & $\sigma_{e l}, 77$ & 1.65 \\
\hline 8 & $Q_{\mathbb{R}}^{2}\left(\mathrm{GeV}^{2}\right)$ & 0.0 & fixo & & $\omega(782)$ & $d \sigma_{e l} / d t, 12$ & 0.83 \\
\hline 9 & $Q_{b}^{2}\left(\mathrm{GeV}^{2}\right)$ & 3.9724 & 0.32482 & 3 & $\phi(1020)$ & $\sigma_{e l}, 39$ & 0.98 \\
\hline 10 & $b_{10}\left(\mathrm{GeV}^{1}\right)$ & 2.1251 & $0.73983 \times 10^{-1}$ & & $\phi(1020)$ & $d \sigma_{e l} / d t, 5$ & 0.61 \\
\hline 11 & $b_{11}\left(\mathrm{GeV}^{1}\right)$ & 2.5979 & 0.21451 & 4 & $J / \Psi(3096)$ & $\sigma_{e l}, 29$ & 0.79 \\
\hline 12 & $b_{00}\left(\mathrm{GeV}^{1}\right)$ & 2.6967 & $0.24985 \times 10^{-1}$ & & $J / \Psi(3096)$ & $d \sigma_{e l} / d t, 70$ & 1.92 \\
\hline 13 & $b_{01}\left(\mathrm{GeV}^{1}\right)$ & 6.7897 & $0.18717 \times 10^{-1}$ & & mésons & No. pontos & $\chi^{2} / \mathrm{DOF}$ \\
\hline 14 & $b_{\mathbb{R}}\left(\mathrm{GeV}^{2}\right)$ & 4.5741 & $0.10509 \times 10^{-2}$ & & $\rho, \omega, \phi, J / \Psi$ & 357 & 1.49 \\
\hline
\end{tabular}

TABela 4.1: Parâmetros obtidos pelo ajuste dos dados experimentais para a fotoprodução dos mésons $\rho, \omega, \phi$ e $J / \Psi$ realizado na Ref. [152].

\subsubsection{Seção de choque total}

Nesta etapa o principal resultado foi a obtenção da seção de choque de fotoprodução total do próton com a inclusão das seções de choque de fotoprodução parciais dos mésons vetoriais. Uma comparação entre as seções de choque de fotoprodução obtidas com o modelo e dados experimentais obtido com o CRISP é mostrada na figura 4.2. 


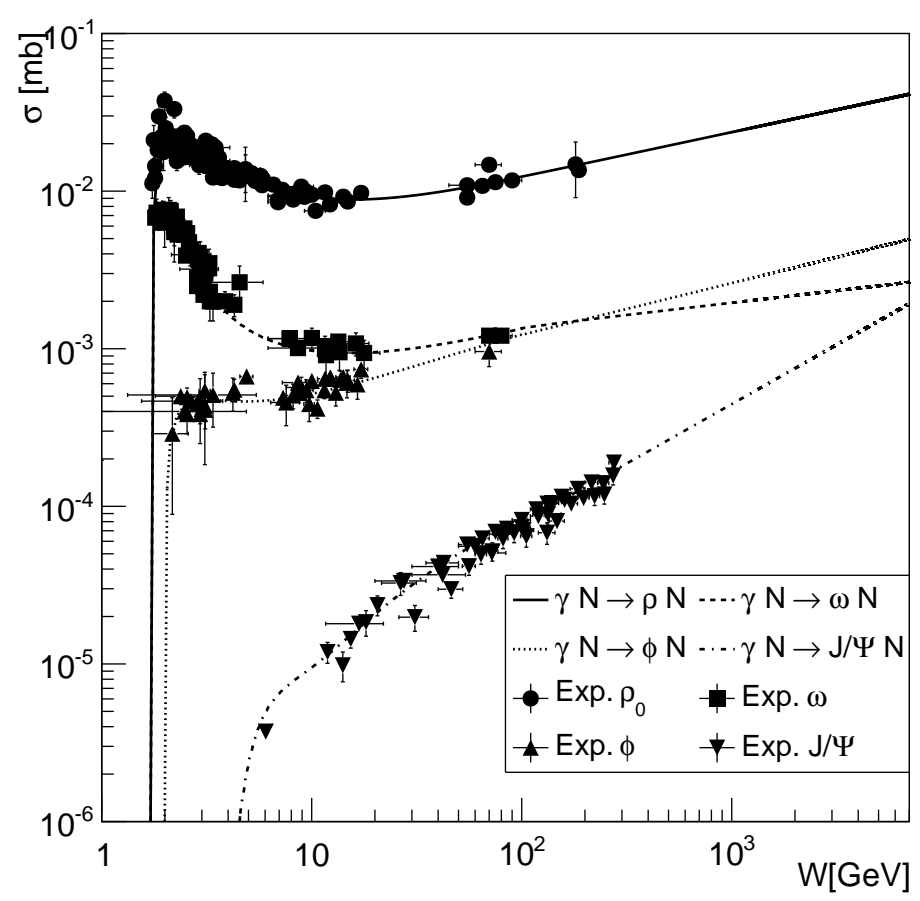

Figura 4.2: Seções de choque de fotoprodução por nucleon obtido gerada com o código CRISP em comparação com dados experimentais obtidos de várias fontes.

\subsubsection{Seção de choque diferencial}

A interação do estado final dos mésons vetoriais depende em grande medida da energia obtida no processo de produção. Esta energia é muito sensível ao momento transferido $t$ da reação inversa. Assim uma correta descrição da seção de choque diferencial

$$
\frac{d \sigma}{d t}=4 \pi|A(s, t)|^{2}
$$

cria as bases para uma boa descrição na simulação do processo de fotoprodução no código CRISP. Em nosso caso usando a amplitude do modelo de soft Pomeron a seção de choque de fotoprodução de mésons vetoriais para o proton é:

$$
\frac{d \sigma}{d t}=4 \pi\left|A\left(z, t ; \tilde{Q}^{2}, M_{V}^{2}\right)\right|^{2}
$$

O processo de simulação produção dos mésons vetoriais induzidos por fótons é simulado com o código CRISP para várias energias para testar a distribuição de momento transferido par cada méson. O resultado é mostrado nas figuras 4.3 e 4.4 comparada com dados experimentais obtido no HERA [159-162]. 

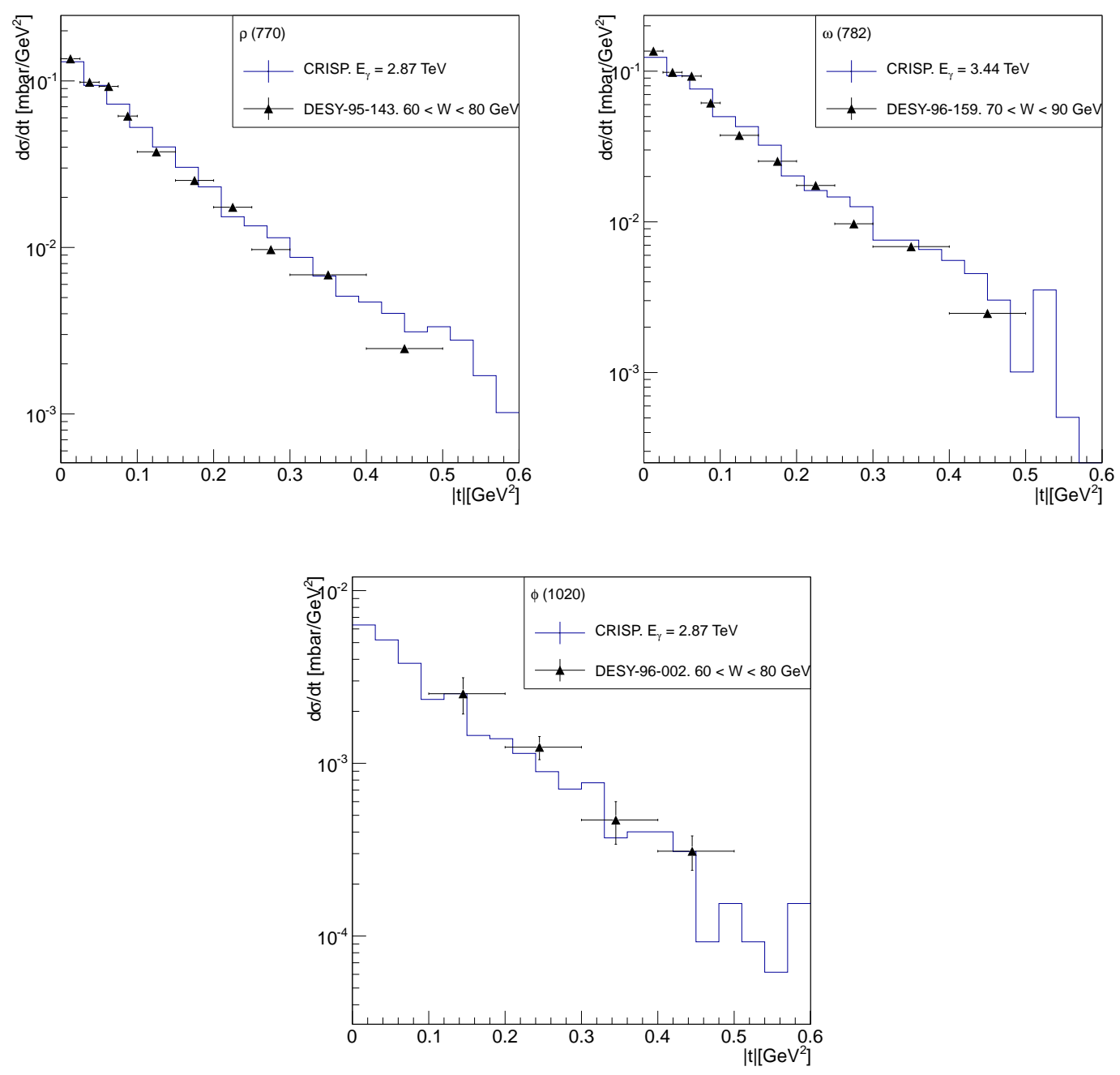

Figura 4.3: Seção de choque diferencial de fotoprodução do méson $\rho_{0}, \omega$ e $\phi$ no póton obtida com o código CRISP comparada com dados experimetais [159-161].

\subsection{Interações no estado final (FSI) dos mésons}

Nesta seção é apresentado o cálculo das interações que acontecem no estado final dos mésons vetoriais na matéria nuclear. As propriedades dos hádrons no meio nuclear são de grande interesse na física nuclear para explicar, por exemplo, a questão da restauração da simetria quiral $[13,163,164]$. Nas altas energias os efeitos colectivos podem ser desconsiderados já que a interação forte é a dominante que tem um range de interação pequeno comparada com as outras interações. Assim, o fenômeno da interação no estado final das partículas no ambiente nuclear pode ser substituído pelo conjunto de colisões binarias como primeira aproximação. No entanto, a FSI será mais precisa de acordo a quantidade de canais incluídos na colisão de dois tipos de partículas. 

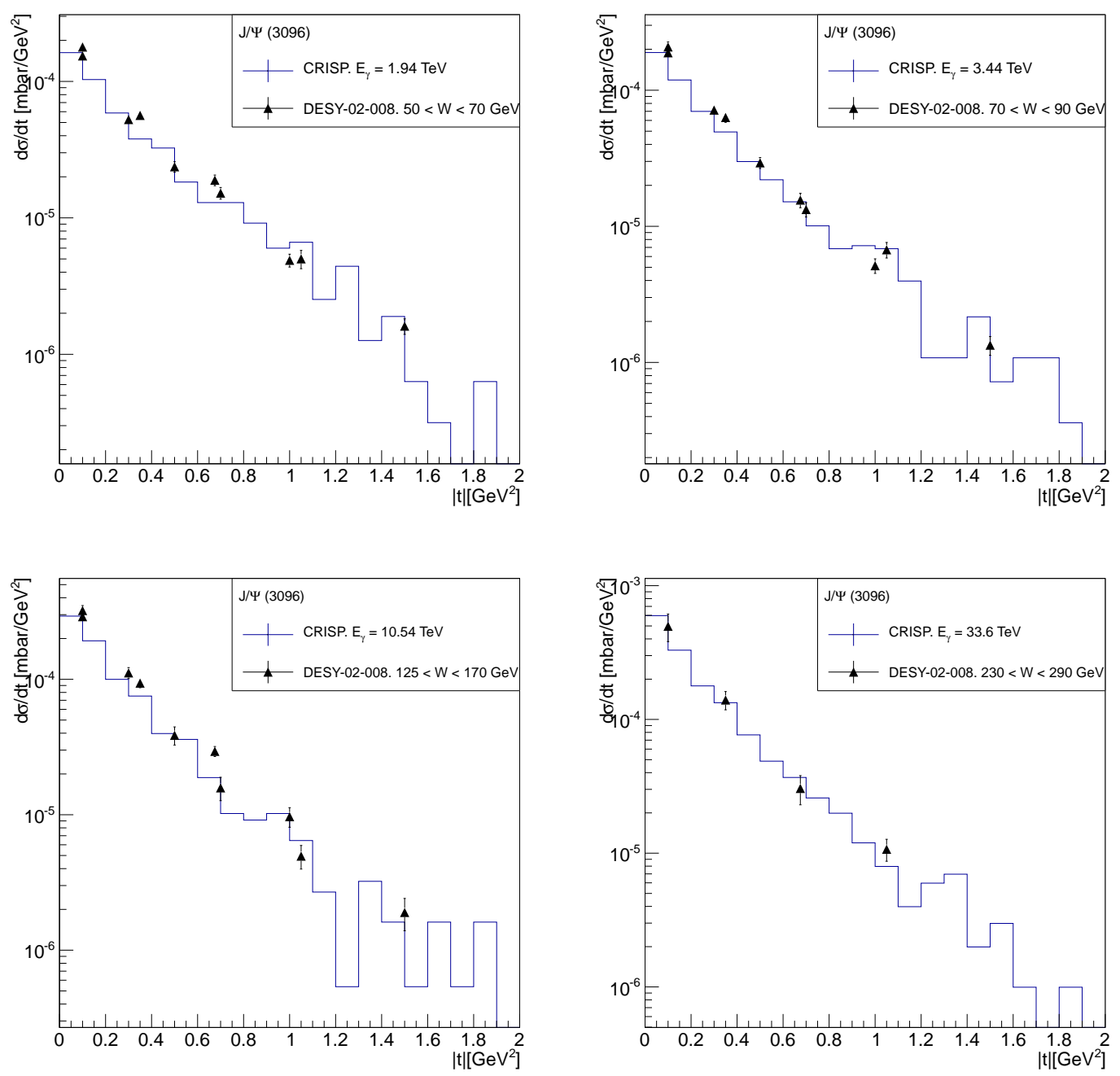

FigurA 4.4: Seção de choque diferencial de fotoprodução do méson $J / \Psi$ no póton obtida com o código CRISP comparada com dados experimetais [162].

Neste trabalho foram usados dois modelos para a inclusão de vários canais nas reações nucleares entre duas partículas no código CRISP o modelo de troca de um méson e o modelo de ressonância. Ao mesmo tempo são calculados os canais inversos usando o principio de balanço detalhado. Estes dois modelos, junto com o principio de balanço detalhado, cobrem a maioria das interações que formam parte da FSIs dos mésons vetoriais no ambiente nuclear.

\subsubsection{O modelo de troca de um méson no estado final da reação $\omega N$}

Os cálculos elástico e inelástico das reações $\omega N \rightarrow \omega N, \rightarrow \pi N, \rightarrow \rho N, \rightarrow \pi \pi N$, foram feitos usando a aproximação troca de bóson, onde a constante de acoplamento $\omega \rho \pi$ e o fator de forma são fixados pela reação $\pi N \rightarrow \omega N$ em comparação com os 


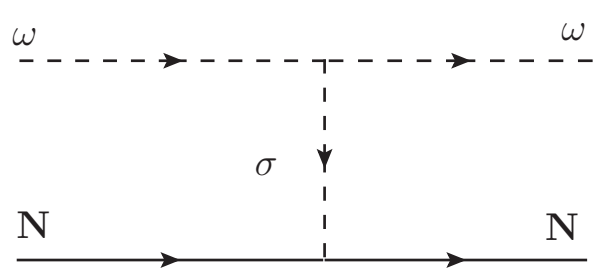

a)

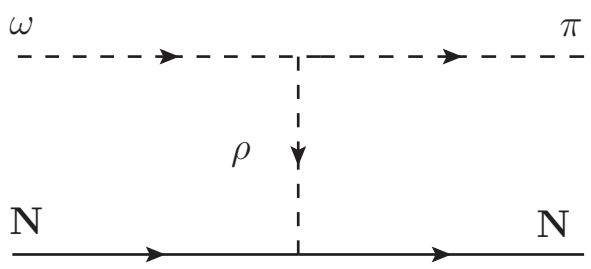

c)

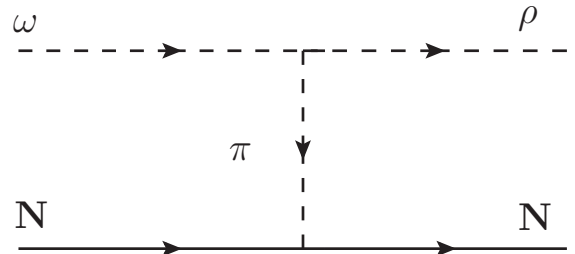

b)

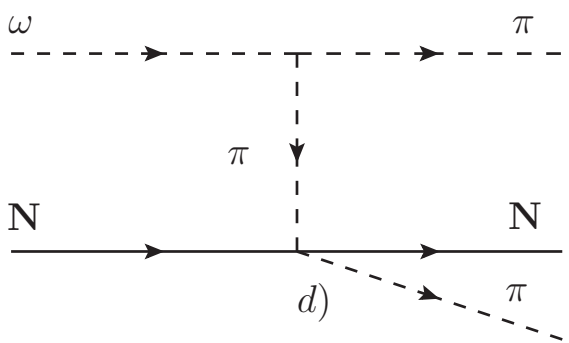

FigurA 4.5: Diagramas dos canais da interação no estado final dos sistema $\omega N$ incluídos no código CRISP.

dados experimentais [165]. Procurando a simplificação do modelo, é adotado o modelo de dominância $\omega \rho \pi$ proposto por Gell-Mann, Sharp e Wagner [166] e a aproximação de troca do méson $\sigma$ que conta como uma troca efetiva de dois méson $\pi$ no canal spinisospin $=0$. Os canais incluídos no código CRISP das interações da FSI usando este modelo são mostrados na figura 4.5. A Lagrangiana do vértice $\omega \rho \pi$ é construída a partir das identidades entre as partes isoescalar e isovetorial das correntes electromagnéticas e mesônicas [21].

Canal elástico $\omega N \rightarrow \omega N$. O canal elástico $\omega N \rightarrow \omega N$ é mostrado no diagrama da figura 4.5 a) e é calculado a través do modelo da troca de um méson $\sigma$. A Lagrangiana usada nesta reação

$$
\begin{aligned}
\mathcal{L}_{\sigma N N} & =g_{\sigma N N} \bar{N} N \cdot \sigma \\
\mathcal{L}_{\omega \sigma \omega} & =g_{\omega \sigma \omega}\left(\partial^{\alpha} \omega^{\beta} \partial_{\alpha} \omega_{\beta}-\partial^{\alpha} \omega^{\beta} \partial_{\beta} \omega_{\alpha}\right) \sigma
\end{aligned}
$$

onde a constante de acoplamento $g_{\sigma N N}=10.54$ e o fator de forma de monopolo terá o parâmetro de corte $\Lambda=2 \mathrm{GeV}$ no vértice $\sigma N N$ [167]. O maior valor da constante de acoplamento é obtido pelo decaimento $\omega \rightarrow 2 \pi^{0} \gamma$ assumindo que este acontece via o decaimento $\omega \rightarrow \omega \sigma$, que a sua vez decaem $\omega \rightarrow \gamma$ e $\sigma \rightarrow 2 \pi^{0}$ respectivamente. Outros valores de constante de acoplamento deste decaimento podem ser encontrados nas Ref. [168-171]. Outros trabalhos calculam esta grandeza a partir da largura do decaimento como $g_{\omega \sigma \omega}=5.7$ obtido usando os dados na Ref. [172] e $g_{\omega \sigma \omega=0.5}$ da Ref. [173]. O modelo [165] usado neste trabalho assume o valor da constante de acoplamento para o vértice $g_{\omega \sigma \omega}=1.76$, mas adverte que não 
há restrição na escolha deste valor e no cálculo é assumido como um parâmetro essencialmente livre. Com este valor as contribuições elástica e inelásticas à seção de choque $\omega N$ estão bastantes equilibradas [165].

A seção de choque elástica diferencial para a reação $\omega N \rightarrow \omega N$ é

$$
\frac{d \sigma}{d t}=\frac{g_{\sigma N N}^{2} g_{\omega \sigma \omega}^{2}}{16 \pi m_{\omega}^{2} \lambda\left(s, m_{\omega}^{2}, m_{N}^{2}\right)}\left(4 m^{2}-t\right) \frac{F_{\omega \sigma \omega}^{2} F_{\sigma N N}^{2}}{\left(t-m_{\sigma}^{2}\right)^{2}}\left(m_{\sigma}^{4}-\frac{m_{\omega}^{2}}{3}+\frac{t^{2}}{12}\right)
$$

com $m_{\sigma}=550 \mathrm{MeV}$ que para baixas valores de momento do méson $\omega$ resulta em $\sigma \approx 15 \mathrm{mb}$. Para maiores valores de momento o modelo cai muito rápido comparado com o valor limite obtido usando o modelo dos quarks $[174,175]$, onde

$$
\sigma_{\omega N \rightarrow \omega N}(s)=\frac{1}{2}\left[\sigma_{\pi^{+} N \rightarrow \pi^{+} N}(s)+\sigma_{\pi^{-} N \rightarrow \pi^{-} N}(s)\right] .
$$

Os autores interpolam o resultado do modelo da troca do méson $\sigma$ com este limite [165] obtendo

$$
\sigma_{\text {elástica }}=5.4+10 \exp \left(-0.6 p_{\omega}\right)[\mathrm{mb}] .
$$

Os resultado da seção de choque elástica pelo modelo da troca de um méson, o limite pelo modelo de quark e a interpolação dos dois modelos são mostrado na figura 4.6.

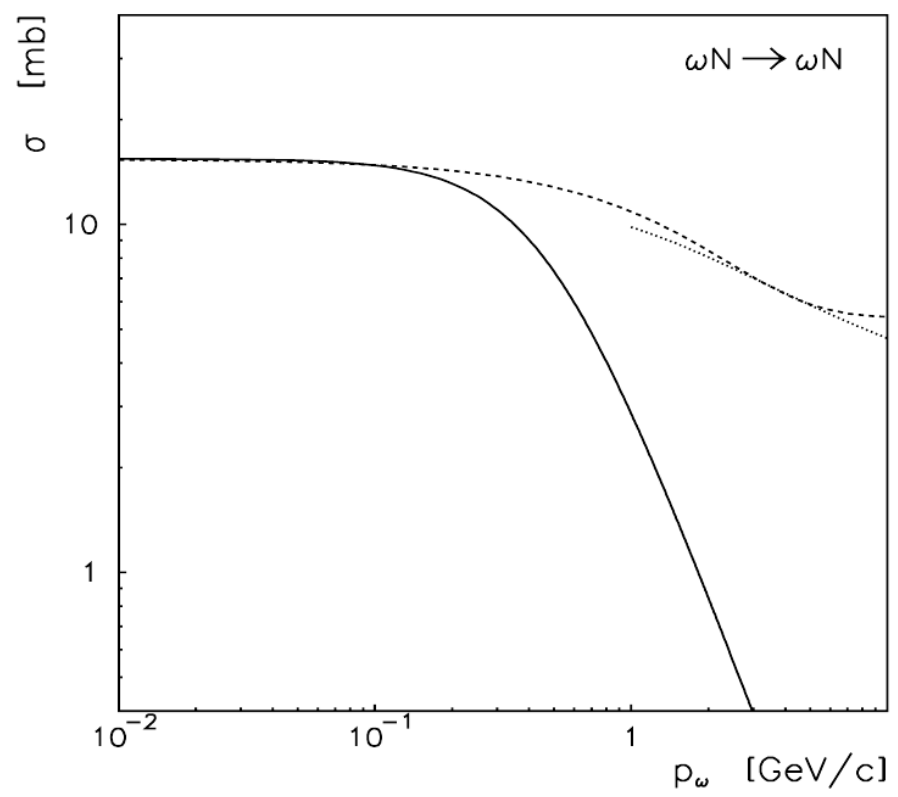

Figura 4.6: Seção de choque elástica $\omega N \rightarrow \omega N$. O modelo da troca de um méson com $g_{\omega \sigma \omega}=1.76$ é representado pela línea cheia, o limite dado pelo modelo de quark pela línea pontilhada e a interpolação dos dois modelos pela línea tracejada. Figura extráida da Ref. [165]. 
Canal $\omega N \rightarrow \pi N$. A Lagrangiana do canal inverso $\pi N \rightarrow \omega N$ mostrado no item $b$ ) da figura 4.5 neste caso é a soma dos termos associados aos vértices $\omega \rho \pi$ e $\rho N N$ [165]

$$
\begin{aligned}
\mathcal{L} & =\mathcal{L}_{\omega \rho \pi}+\mathcal{L}_{\rho N N} \\
\mathcal{L}_{\omega \rho \pi} & =-\frac{g_{\omega \rho \pi}}{m_{\omega}} \epsilon_{\alpha \beta \gamma \delta} \partial^{\alpha} \rho^{\beta} \partial^{\gamma} \omega^{\delta} \pi \\
\mathcal{L}_{\rho N N} & =-g_{\rho N N}\left(\bar{N} \gamma^{\mu} \tau N \rho_{\mu}+\frac{\kappa}{2 m_{N}} \bar{N} \sigma^{\mu \nu} \tau N \partial_{\mu} \rho_{\nu}\right)
\end{aligned}
$$

onde a constante de acoplamento $g_{\omega \rho \pi}=11.79$ foi obtida do decaimento $\omega \rightarrow$ $3 \pi$ [166], $\epsilon_{\alpha \beta \gamma \delta}$ é o tensor antissimétrico, e $\rho, \omega$ e $\pi$, são os campos mesônicos respectivamente. Na Lagrangiana $\rho N N, N$ se refere ao campo nucleônico, $\tau$ são as matrizes de Pauli, a constante $g_{\rho N N}=3.24$ foi extraída da referência [176], a constante do tensor de acoplamento $\kappa=f_{\rho N N} / g_{\rho N N}=6.1$.

De acordo à Lagrangiana, a seção de choque para o processo $\pi N \rightarrow \omega N$ é

$$
\begin{aligned}
& \frac{d \sigma}{d t}=\frac{g_{\omega \rho \pi}^{2}}{m_{\omega}^{2}} \frac{1}{8 \pi \lambda\left(s, m_{N}^{2}, m_{\pi}^{2}\right)} \frac{F_{\omega \rho \pi}^{2} F_{\rho N N}^{2}}{\left(t-m_{\rho}^{2}\right)^{2}} \\
& \times\left[-\left(g_{\rho N N}+f_{\rho N N}\right)^{2} m_{\omega}^{2} q_{\omega}^{2} t+\left(g_{\rho N N}^{2}-\frac{f_{\rho N N}^{2} t}{4 m_{N}^{2}}\right)\left(\frac{\sin ^{2} \theta}{8 s} \lambda\left(s, m_{N}^{2}, m_{\pi}^{2}\right) \lambda\left(s, m_{N}^{2}, m_{\omega}^{2}\right)\right)\right]
\end{aligned}
$$

onde $q_{\omega}^{2}=\frac{\lambda\left(t, m_{\omega}^{2}, m_{\pi}^{2}\right)}{4 m_{\omega}^{2}}, s$ é a energia de colisão invariante ao quadrado, $t$ é o momento transferido ao quadrado, $\theta$ é o ângulo de emissão e $\lambda(x, y, z)$ é a função triangular. Para o vértice $\omega \rho \pi$ é usado o fator de forma [165]

$$
F(t)=\frac{\Lambda^{2}-m_{\rho}^{2}}{\Lambda^{2}-t}
$$

Para o vértice $\rho N N$ é usado o fator de forma mais geral, devido às discrepâncias entre cálculos feitos com um fator de forma similar ao vértice $\omega \rho \pi$ e os dados experimentais, usando o modelo de intercâmbio de um méson $\rho$. Este fator de forma é proposto

$$
F(t, s)=\exp (\beta t) \exp (-\alpha s)
$$

O ajuste da seção de choque aos dados experimentais resultaram nos seguintes valores dos parâmetros [165]

$$
\Lambda=2.7 \mathrm{GeV}, \quad \beta=2.3 \mathrm{GeV}^{-2}, \quad \alpha=0.16 \mathrm{GeV}^{-2}
$$

Os resultados dos cálculos expostos na referência [165], são mostrados nas figuras 4.7, 4.8, 4.9, 4.10 para as reações $\pi^{+} n \rightarrow \omega p$ e $\pi^{-} p \rightarrow \omega n$ para um valor de 
momento fixo referente ao sistema de coordenadas do laboratório.

Canal $\omega N \rightarrow \rho N$.

O canal $\omega N \rightarrow \rho N$ é calculado de acordo com o diagrama mostrado no item $c$ ) da figura 4.5. A lagrangiana para o vértice $\omega \rho \pi$ é igual o canal anterior, mas o termo da lagrangiana de interação associado ao vértice $\pi N N$ é

$$
\mathcal{L}_{\pi N N}=-g_{\pi N N} \bar{N} \gamma_{5} \tau N \cdot \pi
$$

onde $g_{\pi N N}=13.59$ é a constante de acoplamento [177]. A seção de choque diferencial é

$$
\frac{d \sigma}{d t}=-g_{\pi N N}^{2} \frac{g_{\omega \rho \pi}^{2}}{m_{\omega}^{2}} t \frac{\left(t-m_{\omega}^{2}-m_{\rho}^{2}\right)^{2}-4 m_{\omega}^{2} m_{\rho}^{2}}{96 \pi \lambda\left(s, m_{\omega}^{2}, m_{N}^{2}\right)} \times \frac{F_{\omega \rho \pi}^{2}(t) F_{\pi N N}^{2}(t)}{\left(t-m_{\pi}^{2}\right)^{2}}
$$
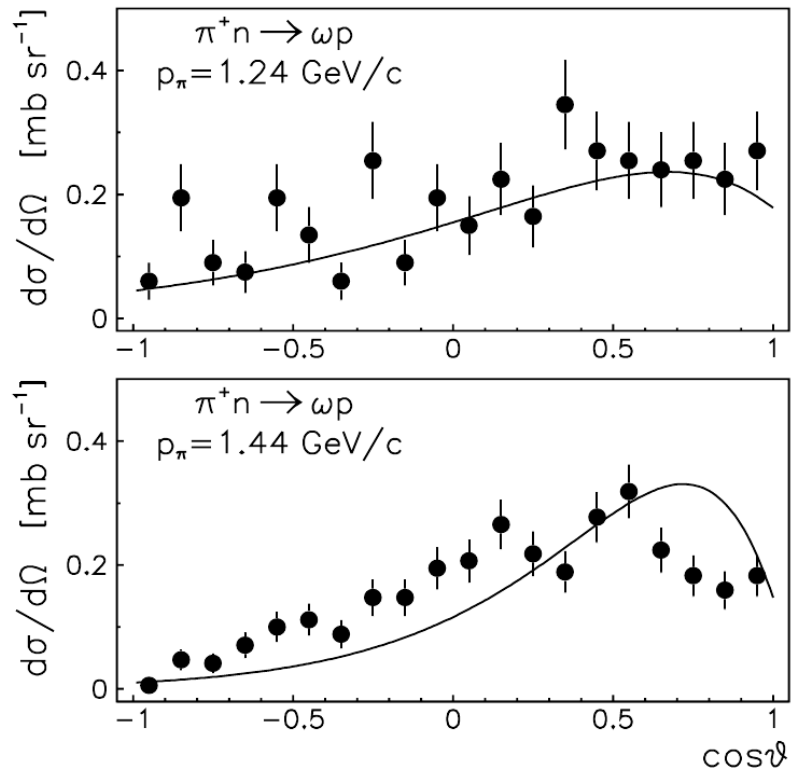

FigurA 4.7: Seções de choque diferenciais angular para $\pi^{+} n \rightarrow \omega p$. Figura extráida da Ref. [165].

onde a constante de acoplamento e o fator de forma do vértice $\omega \rho \pi$ é fixado pelo canal $\pi N \rightarrow \omega N$. O fator de forma do vértice $\pi N N$ é o mesmo que da equação 4.27 usando $\lambda=1.05$ de acordo com a referência [178]. 

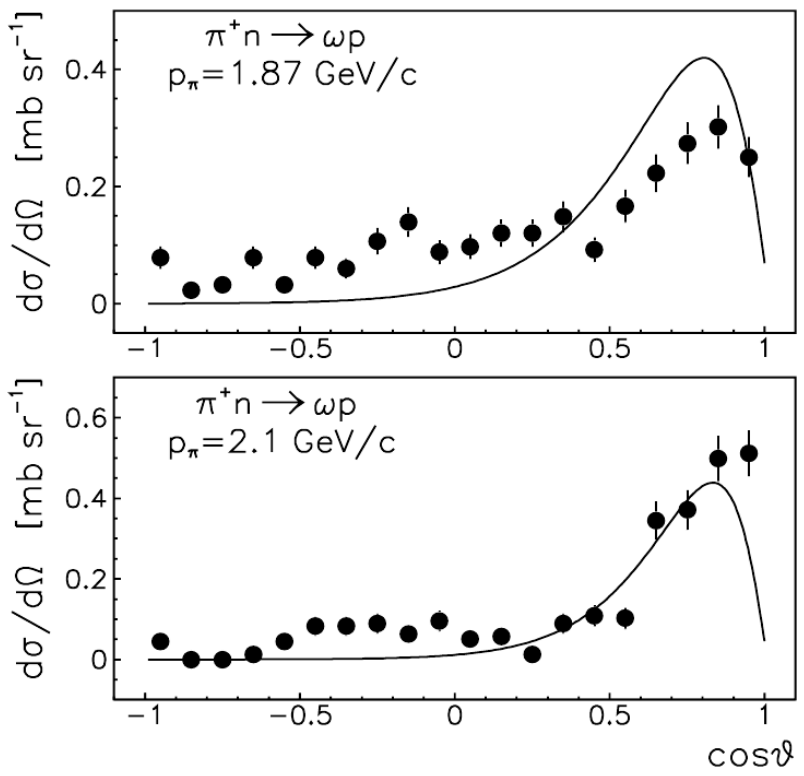

Figura 4.8: Seções de choque diferenciais angular para $\pi^{+} n \rightarrow \omega p$. Figura extráida da Ref. [165].
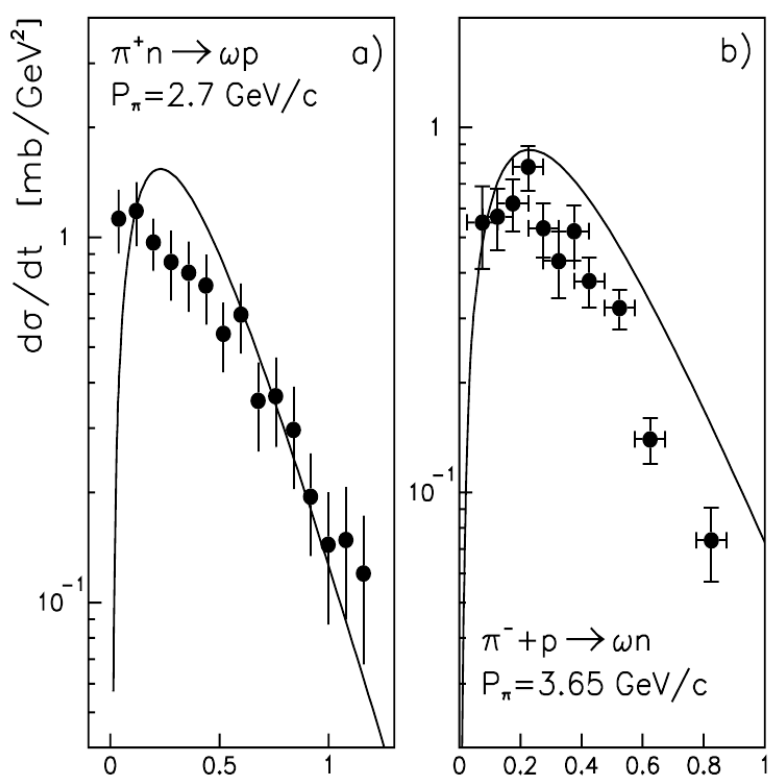

Figura 4.9: Seções de choque diferenciais para $\pi^{+} n \rightarrow \omega p$ e $\pi^{-} p \rightarrow \omega n$. Figura extráida da Ref. [165]. 

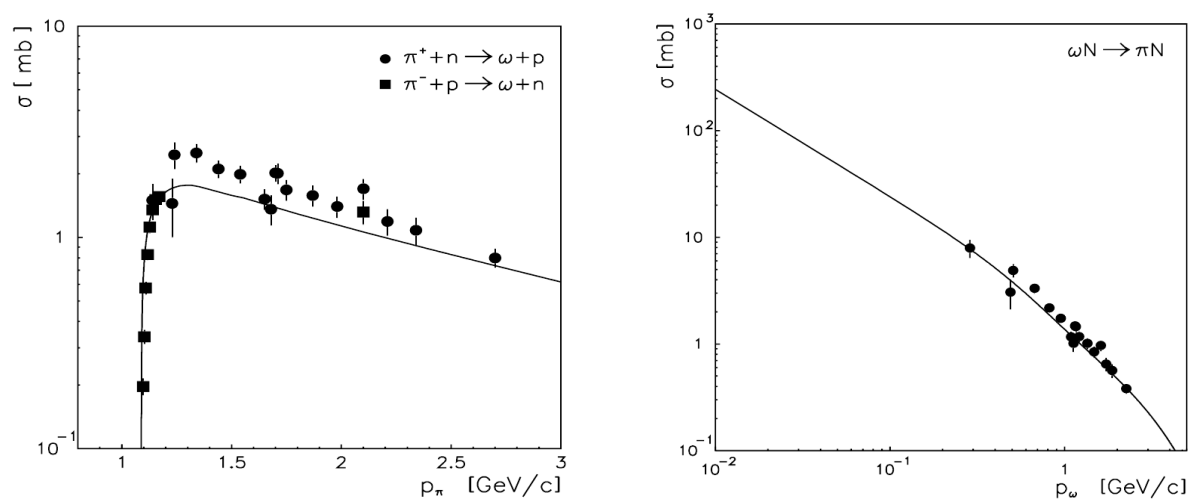

Figura 4.10: Seções de choque totais para os canais $\pi N \rightarrow \omega N$ e $\omega N \rightarrow \pi N$. Figura extráida da Ref. [165].

Canal $\omega N \rightarrow 2 \pi N$. O canal $\omega N \rightarrow 2 \pi N$ é calculado de acordo com o diagrama mostrado na figura $4.5 d$ ) e é dada por

$$
\begin{aligned}
\sigma & =\frac{1}{32 \pi^{2} \lambda\left(s, m_{\omega}^{2}, m_{N}^{2}\right)} \int_{\left(m_{N}+m_{\pi}\right)^{2}}^{\left(\sqrt{s}-m_{\pi}\right)^{2}} d s_{1} \lambda^{1 / 2}\left(s_{1}, m_{\rho}^{2}, m_{N}^{2}\right) \\
& \times \sigma_{\rho N \rightarrow \pi N}\left(s_{1}\right) \int_{t_{-}}^{t_{+}} d t \frac{g_{\omega \rho \pi}^{2} F_{\omega \rho \pi}^{2}}{m_{\omega}^{2}\left(t-m_{\rho}^{2}\right)^{2}}\left[\left(t+m_{\omega}^{2}-m_{\pi}^{2}\right)^{2}-4 m_{\omega}^{2} t\right]
\end{aligned}
$$

onde

$t^{ \pm}=m_{\omega}^{2}+m_{\pi}^{2}-\frac{1}{2 s}\left[\left(s+m_{\omega}^{2}-m_{N}^{2}\right)\left(s+m_{\pi}^{2}-s_{1}\right) \mp \lambda^{1 / 2}\left(s, m_{\omega}^{2}, m_{N}^{2}\right) \lambda^{1 / 2}\left(s, m_{\pi}^{2}, s_{1}\right)\right]$.

A seção de choque $\sigma_{\rho N \rightarrow \pi N}$ é a seção de choque do canal $\rho N \rightarrow \pi N$ que é calculada pelo princípio de balanço detalhado da reação inversa, usando o modelo ressonante descrito mais abaixo na seção 4.2 .3

$$
\sigma_{\rho N \pi N}=\frac{\pi^{2}}{3} \frac{a}{\lambda^{1 / 2}\left(s_{1}, m_{\rho}^{2}, m_{N}^{2}\right)} \frac{\Gamma^{2}}{\left(\sqrt{s_{1}}-M\right)^{2}+\Gamma^{2} / 4}
$$

onde os parâmetros $a=413 \mu b / \mathrm{GeV}^{2}, M=1.809 \mathrm{GeV}$ e $\Gamma=0.99 \mathrm{GeV}$.

\subsubsection{O modelo de troca de um méson para a dissociação do méson $J / \Psi$}

A dissociação do méson $J / \Psi$ no ambiente nuclear é dada pela colisão com nucleons. Este processo de dissociação é dado pelas reações $J / \Psi N \rightarrow \Lambda_{c} \bar{D}, \rightarrow \Lambda_{c} \bar{D}^{*}$ e $\rightarrow N D \bar{D}$ que são mostrados nos diagramas $a), b), c$ ) e $d$ ) da figura 4.11 . 


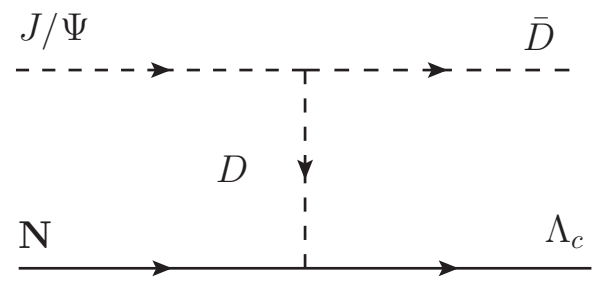

a)

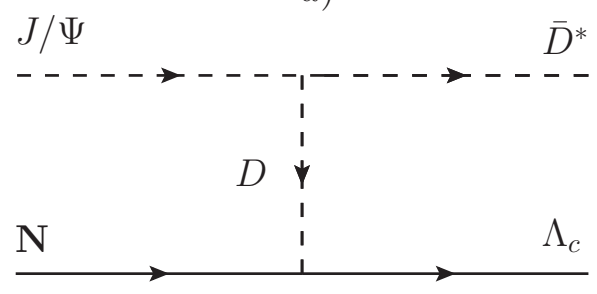

c)

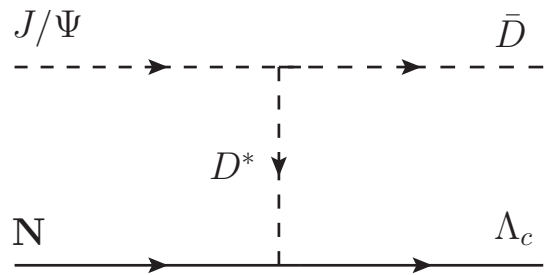

b)

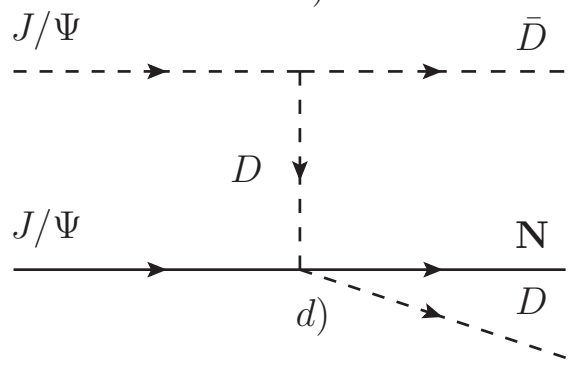

Figura 4.11: Processo de dissociação das interações $J / \Psi N \rightarrow X_{1} X_{2}$ incluídas no código CRISP.

Para incluir este processo no CRISP foi usado o modelo de troca de um bóson proposto A. Sibirtsev, K. Tsushima e A. W. Thomas [179]. O modelo tem a base experimental reportada pela colaboração NA50 para a supressão do méson $J / \Psi$ em colisões $\mathrm{Pb}+\mathrm{Pb}$ no CERN-SPS [180]. Este modelo é consistente com cálculos de primeira ordem de QCD nas altas energias, mas reporta diferenças perto do limiar da reação. O modelo usa as seguintes densidades Lagrangianas de interação [179]

$$
\begin{aligned}
\mathcal{L}_{J D D} & =i g_{J D D} J^{\mu}\left[\bar{D}\left(\partial_{\mu} D\right)-\left(\partial_{\mu} \bar{D}\right) D\right], \\
\mathcal{L}_{D N \Lambda_{c}} & =i g_{D N \Lambda_{c}}\left[\bar{N} \gamma_{5} \Lambda_{c} D+\bar{D} \bar{\Lambda}_{c} \gamma_{5} N\right], \\
\mathcal{L}_{J D^{*} D} & =\frac{g_{J D^{*} D}}{m_{J}} \varepsilon_{\alpha \beta \mu \nu}\left(\partial^{\alpha} J^{\beta}\right)\left[\left(\partial^{\mu} \bar{D}^{* \nu}\right) D+\bar{D}\left(\partial^{\mu} D^{* \nu}\right)\right], \\
\mathcal{L}_{D^{*} N \Lambda_{c}} & =-g_{D^{*} N \Lambda_{c}}\left[\bar{N} \gamma_{\mu} \Lambda_{c} D^{* \mu}+\bar{D}^{* \mu} \bar{\Lambda}_{c} \gamma_{\mu} N\right],
\end{aligned}
$$

onde

$$
N=\left(\begin{array}{c}
p \\
n
\end{array}\right), \bar{N}=N^{\dagger} \gamma_{0}, \quad D \equiv\left(\begin{array}{c}
D^{0} \\
D^{+}
\end{array}\right), \bar{D}=D^{\dagger}
$$

Uma notação similar é usada para $D^{*}$ e $\bar{D}^{*}$. Os vértices de interação possuem uma contante de acoplamento $g_{J D D}=g_{J D D^{*}}=7.64, g_{D N \Lambda_{c}}=14.8, g_{D^{*} N \Lambda_{c}}=-19$ e estão associados a um fator de forma parametrizado segundo um monopolo convencional

$$
F(t)=\frac{\Lambda^{2}}{\Lambda^{2}-t}
$$

onde $t$ é o momento transferido. $\Lambda$ é o parâmetro de corte e tem o valor fixo $\Lambda=3.1$ para os vértices $J D D$ e $J D D^{*}$; enquanto $\Lambda=2$ para os vértices $D N \Lambda_{c}$ e $D^{*} N \Lambda_{c}$. 
As seções de choque diferenciais dos processos representados nos diagramas $a, b, c$ da Figura 4.11 no sistema de coordenadas do centro de massa são

$$
\begin{aligned}
& \frac{d \sigma_{a, b}}{d \Omega}=\frac{1}{64 \pi^{2} s}{\overline{\mid M_{e, f}}}^{2}\left(\frac{\left[\left(m_{\Lambda}+m_{D}\right)^{2}-s\right]\left[\left(m_{\Lambda}-m_{D}\right)^{2}-s\right]}{\left[\left(m_{N}+m_{J / \Psi}\right)^{2}-s\right]\left[\left(m_{N}-m_{J / \Psi}\right)^{2}-s\right]}\right)^{1 / 2} \\
& \frac{d \sigma_{c}}{d \Omega}=\frac{1}{64 \pi^{2} s}{\overline{\mid M_{g}}}^{2}\left(\frac{\left[\left(m_{\Lambda}+m_{D^{*}}\right)^{2}-s\right]\left[\left(m_{\Lambda}-m_{D^{*}}\right)^{2}-s\right]}{\left[\left(m_{N}+m_{J / \Psi}\right)^{2}-s\right]\left[\left(m_{N}-m_{J / \Psi}\right)^{2}-s\right]}\right)^{1 / 2},
\end{aligned}
$$

onde $s$ é a energia do centro de masas ao quadrado e os termos ${\overline{\left|M_{e, f, g}\right|}}^{2}$ são as amplitudes ao quadrado de cada processo, tomando a média sobe os spins iniciais e somada sobe os spins finais. Estas amplitudes são dadas pelas equações [179]

$$
\begin{aligned}
{\overline{\mid M_{a}}}^{2}= & \frac{8 g_{J D D}^{2} g_{D N \Lambda_{c}}^{2}}{3 m_{J / \Psi}^{2}}\left(\frac{1}{q^{2}-m_{D}^{2}}+\frac{1}{2 p_{J / \Psi} \cdot p_{\bar{D}}}\right)^{2} \\
& \times\left(p_{N} \cdot p_{\Lambda_{c}}-m_{N} m_{\Lambda_{c}}\right)\left[\left(p_{J / \Psi} \cdot p_{\bar{D}}\right)^{2}-m_{J / \Psi}^{2} m_{\bar{D}}^{2}\right] \\
{\overline{\mid M_{b}}}^{2}= & \frac{g_{J D^{*} D}^{2} g_{D^{*} N \Lambda_{c}}^{2}}{3 m_{J / \Psi}^{2}} \frac{1}{\left(q^{2}-m_{D^{*}}^{2}\right)^{2}} \\
& \times\left\{m_{J / \Psi}^{2}\left[p^{2} q^{2}-\left(m_{\Lambda_{c}}^{2}-m_{N}^{2}\right)^{2}\right]+2\left(p_{J / \Psi} \cdot p\right)\left(p_{J / \Psi} \cdot q\right)\left(m_{\Lambda_{c}}^{2}-m_{N}^{2}\right)\right. \\
& \left.-p^{2}\left(p_{J / \Psi} \cdot q\right)^{2}-q^{2}\left(p_{J / \Psi} \cdot p\right)^{2}-4\left(p_{N} \cdot p_{\Lambda_{c}}-m_{N} m_{\Lambda_{c}}\right)\left[m_{J / \Psi}^{2} q^{2}-\left(p_{J / \Psi} \cdot p\right)^{2}\right]\right\} \\
{\overline{\left|M_{c}\right|}}^{2}= & \frac{4 g_{J D^{*} D}^{2} g_{D N \Lambda_{c}}^{2}}{3 m_{J / \Psi}^{2}} \frac{1}{\left(q^{2}-m_{D}^{2}\right)^{2}}\left(p_{N} \cdot p_{\Lambda_{c}}-m_{N} m_{\Lambda_{c}}\right)\left[\left(p_{J / \Psi} \cdot p_{\bar{D}^{*}}\right)^{2}-m_{J / \Psi}^{2} m_{\bar{D}^{*}}^{2}\right]
\end{aligned}
$$

onde $q \equiv p_{J / \Psi}-p_{\bar{D}}=p_{\Lambda_{c}}-p_{N}$ é o momento transferido, $p \equiv p_{\Lambda_{c}}+p_{N}$ e $p_{i}$ é o momento da partícula $i$.

A seção de choque diferencial da segunda ordem para a reação $J / \Psi+N \rightarrow N+D+\bar{D}$, mostrado no diagrama $d$ ) da Figura 4.11, é da forma [179]

$$
\begin{aligned}
\frac{d^{2} \sigma_{d}}{d t d s_{1}} & =\frac{g_{J / \Psi D D}^{2}}{96 \pi^{2} q_{J / \Psi}^{2} s} q_{D} \sqrt{s_{1}} \frac{F^{2}(t)}{\left(t-m_{D}^{2}\right)^{2}} \\
& \times \frac{\left[\left(m_{J / \Psi}+m_{D}\right)^{2}-t\right]\left[\left(m_{J / \Psi}-m_{D}\right)^{2}-t\right]}{m_{J / \Psi}^{2}} \sigma_{D N}\left(s_{1}\right),
\end{aligned}
$$

onde

$$
\begin{aligned}
q_{D}^{2} & =\frac{\left[\left(m_{N}+m_{D}\right)^{2}-s_{1}\right]\left[\left(m_{N}-m_{D}\right)^{2}-s_{1}\right]}{4 s_{1}} \\
q_{J / \Psi}^{2} & =\frac{\left[\left(m_{N}+m_{J / \Psi}\right)^{2}-s\right]\left[\left(m_{N}-m_{J / \Psi}\right)^{2}-s\right]}{4 s} .
\end{aligned}
$$


A seção de choque $D N$ e $\bar{D} N$ é parametrizada da forma [181]

$$
\sigma_{\bar{D} N}\left(s_{1}\right)=\left(\frac{\left[\left(m_{\Lambda_{c}}+m_{\pi}\right)^{2}-s_{1}\right]\left[\left(m_{\Lambda_{c}}-m_{\pi}\right)^{2}-s_{1}\right]}{\left[\left(m_{N}+m_{D}\right)^{2}-s_{1}\right]\left[\left(m_{N}-m_{D}\right)^{2}-s_{1}\right]}\right)^{1 / 2} \times \frac{27}{s_{1}}+20
$$

onde $m_{\Lambda_{c}}$ e $m_{\pi}$ são as massas do hyperon e píon respectivamente.

\subsubsection{O modelo ressonante no estado final da reação $\pi N$}

A seção de choque para a produção dos mésons vetoriais $\rho, \omega, \phi$ induzida por píons é calculada usando o ajuste desenvolvido por A. Sibirtsev, W. Cassing e U. Mosel pelo modelo de ressonância [182] através da reação

$$
\pi+N \rightarrow R \rightarrow M+N
$$

especificamente pelas reações $\pi N \rightarrow N \rho \rightarrow N \omega, \rightarrow N \phi$ como é mostrado na Figura 4.12. Assumindo que o elemento da matriz quadrada é proporcional a função de Breit-

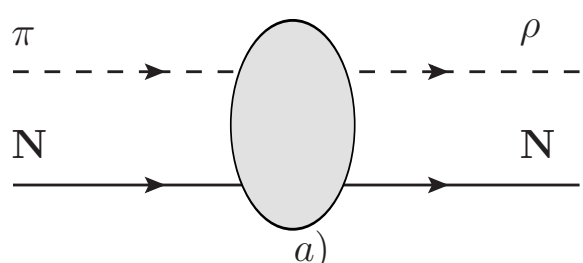

a)

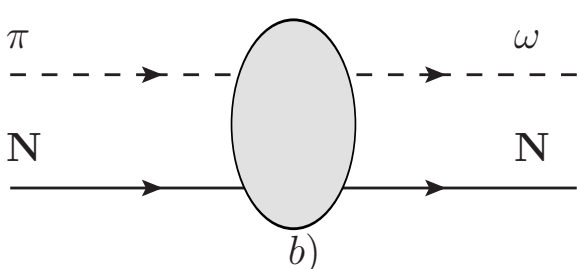

$\phi$

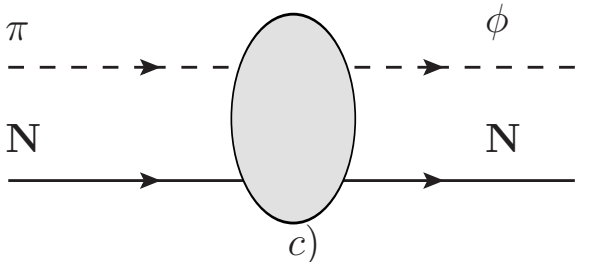

Figura 4.12: Processos de produção de mésons vectoriais pelo modelo ressonante incluídos no código CRISP.

Wigner, a seção de choque é

$$
\sigma_{\pi+N \rightarrow M+N}(s)=\frac{\pi}{k^{2}} \frac{2 J+1}{2} \frac{B_{\text {in }} B_{\text {out }} \Gamma^{2}}{\left(\sqrt{s}-M_{R}\right)^{2}+\Gamma^{2} / 4} \times R_{2}(s)
$$

onde $J, B_{\text {in }}$ e $B_{\text {out }}$ são o spin da ressonância, as taxas das partículas incidentes e produtos, respectivamente. O fator $R_{2}$ se refere ao volume do espaço de fase das partículas finais $R_{2}(s)=\pi \lambda\left(s, m_{M}^{2}, m_{N}^{2}\right) / \sqrt{s}$ e $k^{2}=\lambda\left(s, m_{\pi}^{2}, m_{N}^{2}\right)$. 
Os parâmetros $M_{R}$ e $\Gamma$ são a massa da ressonância e largura, respectivamente. Os valores desse parâmetros correspondente ao melhor ajuste de dados experimentais ao modelo [182] são mostrados na Tabela 4.2.

TABela 4.2: Massa efetiva e largura da ressonância bariônica mostradas em (4.52), e os termos $B=B_{\text {in }} B_{\text {out }}(2 J+1)$ obtido pelo ajuste de datos experimentais à equação de Breit-Wigner na Ref. [182].

\begin{tabular}{cccc}
\hline \hline Meson & $M_{R}(\mathrm{GeV})$ & $\Gamma(\mathrm{GeV})$ & $\mathrm{B}\left(\mu \mathrm{b} \mathrm{GeV}^{-2}\right)$ \\
\hline$\rho^{0}$ & 1.809 & 0.99 & 413 \\
$\omega$ & 1.809 & 0.99 & 302 \\
$\phi$ & 1.8 & 0.99 & 5.88 \\
\hline \hline
\end{tabular}

\subsubsection{Interação no estado final dos mésons vectoriais $\pi, \rho, \omega, \phi$ e $J / \Psi$ incluída no código CRISP}

Nesta seção é apresentado um resumo das seções de choque das reações associadas à interação no estado final dos mésons vectoriais incluídos no código CRISP. A FSI dos mésons vectoriais incluem todas as reações nucleares obtidas pelas abordagem tratadas nas seções anteriores. Inclui também as reações nucleares obtidas do principio de balanço detalhado aplicado à todas interações com duas partículas no estado final.
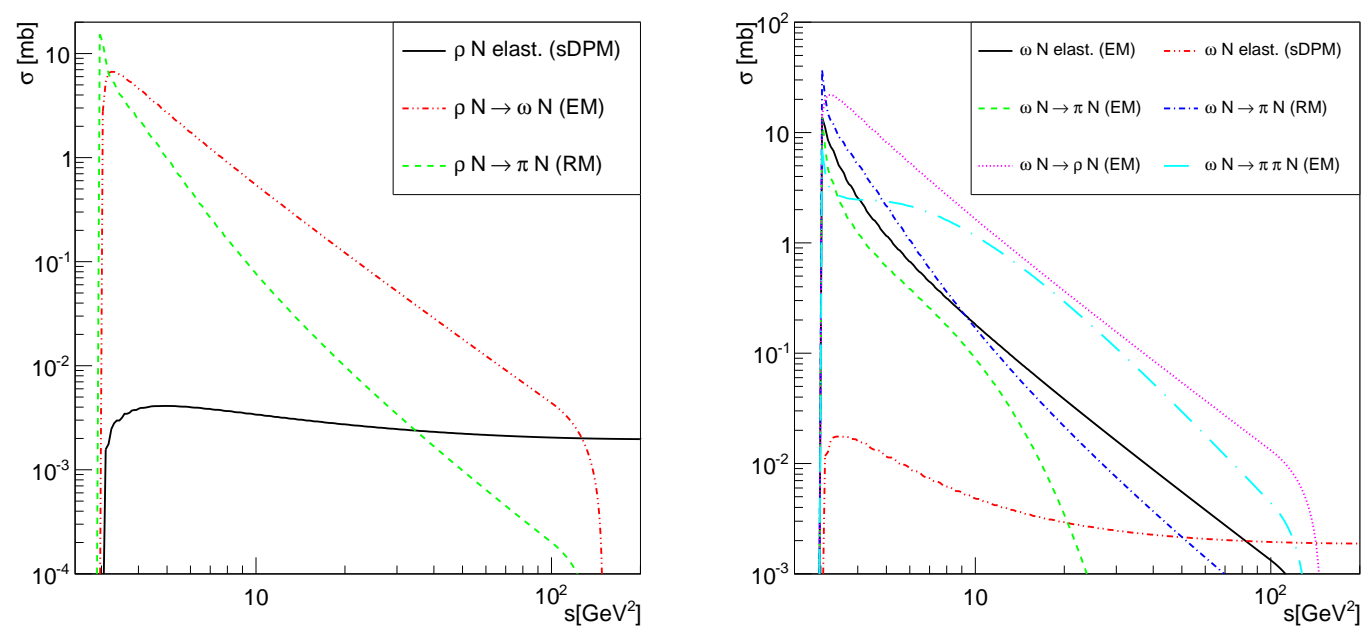

Figura 4.13: Seções de choque totais da interação $\rho N \rightarrow V N$ (esquerda) e $\omega N \rightarrow V N$ (direita) incluídas no CRISP.

As seções de choque em função de $s$ de todas a reações incluída até a presente data no CRISP são mostradas por partículas nas Figuras 4.13 esquerda (méson $\rho$ ), 4.13 direita (méson $\omega$ ), 4.14 ( esquerda méson $\phi$ ), 4.14 direita(méson $J / \Psi$ ) e 4.15 esquerda (méson 
$\pi$ ) de acordo com cada modelo incluído modelo ressonante (RM), modelo da troca de um méson (EM) e o modelo de dipolo de soft Pomeron (sDPM). Este último molelo apresenta uma seção de choque para a interação elástica $V N \rightarrow V N$ cuja amplitude é dada pela equação 4.6. As colisões elásticas incluídas usando este último modelo simulam colisões que são importantes nas altas energias, desde que estas estão determinadas pela troca de reggeons. Como foi discutido no capítulo 3, estas reações são favorecidas quando é transferido um valor de momento pequeno e nas altas energias (reações soft). Um indicativo desta analise é o crescimento da seção de choque com o aumento da energia. Novos modelos que reproduzam corretamente as colisões elásticas dos mésons $\rho, \phi$ e $J / \Psi$ nas energias menores deverão ser incluídos como foi para o méson $\omega$ cuja seção de choque da sua colisão elástica foi descrita pela troca de um méson $\sigma$.
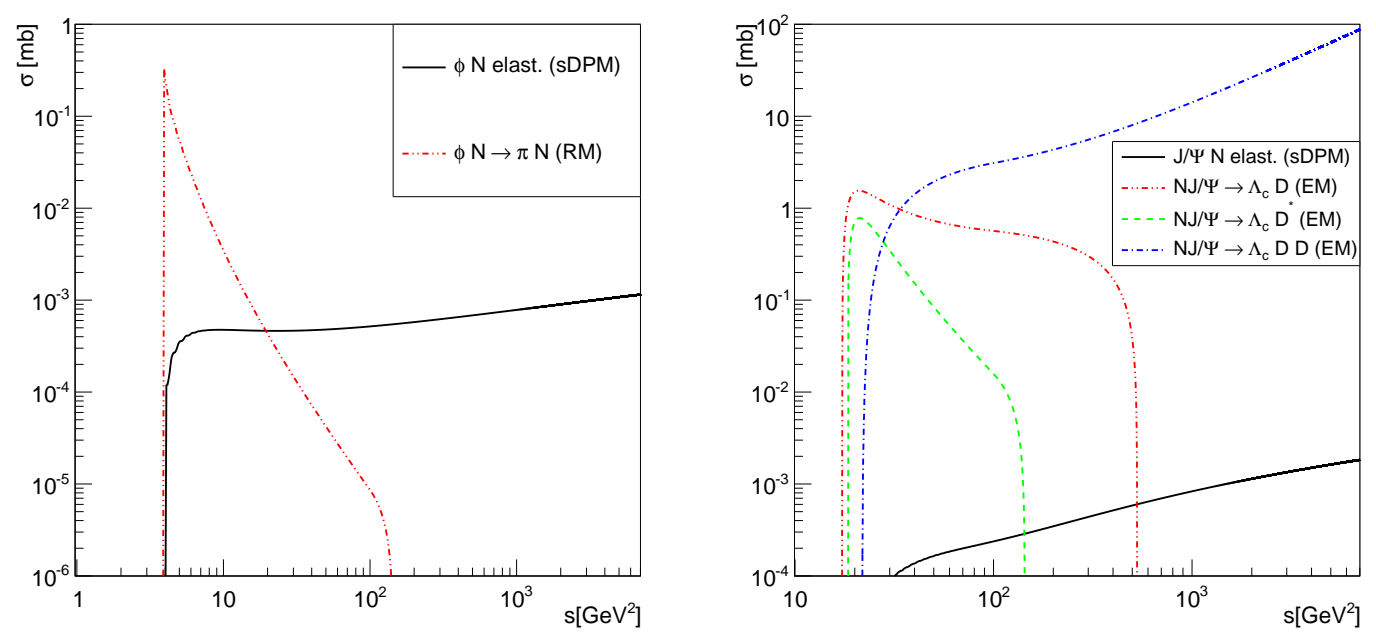

Figura 4.14: Seções de choque totais da interação $\phi N \rightarrow V N$ (esquerda) e $J / \Psi N \rightarrow$ $V N$ (direita) incluídas no CRISP.

Note-se a importância destas reações nucleares na FSI acima do limiar de produção, especialmente nas colisões $\omega N \rightarrow \rho N$ e $\omega N \rightarrow \pi N$. As figuras mostram que seções de choque de interação diminuem com o aumento da energia $s$ com exceção dos processos de dissociação do méson $J / \Psi$ onde a probabilidade de interação aumenta com o incremento da energia $s$. Os mésons vetoriais também podem ser obtidos por interações induzidas por píons.

A figura 4.15 esquerda mostra a seção de choque destas interações que representam a produção dos mésons $\rho, \omega$ e $\phi$ a partir da colisão de um méson $\pi$ com um nucleon.

Note-se que seção de choque da reação $\omega N \rightarrow \pi N$ pode ser calculada a partir de dois modelos: o modelo ressonante e o modelo de troca de um méson. Uma comparação dos cálculos destes modelos pode ser analisada na figura 4.15 direita em comparação com dados experimentais mostrados na figura 5 da Referencia [182]. De acordo à esta 

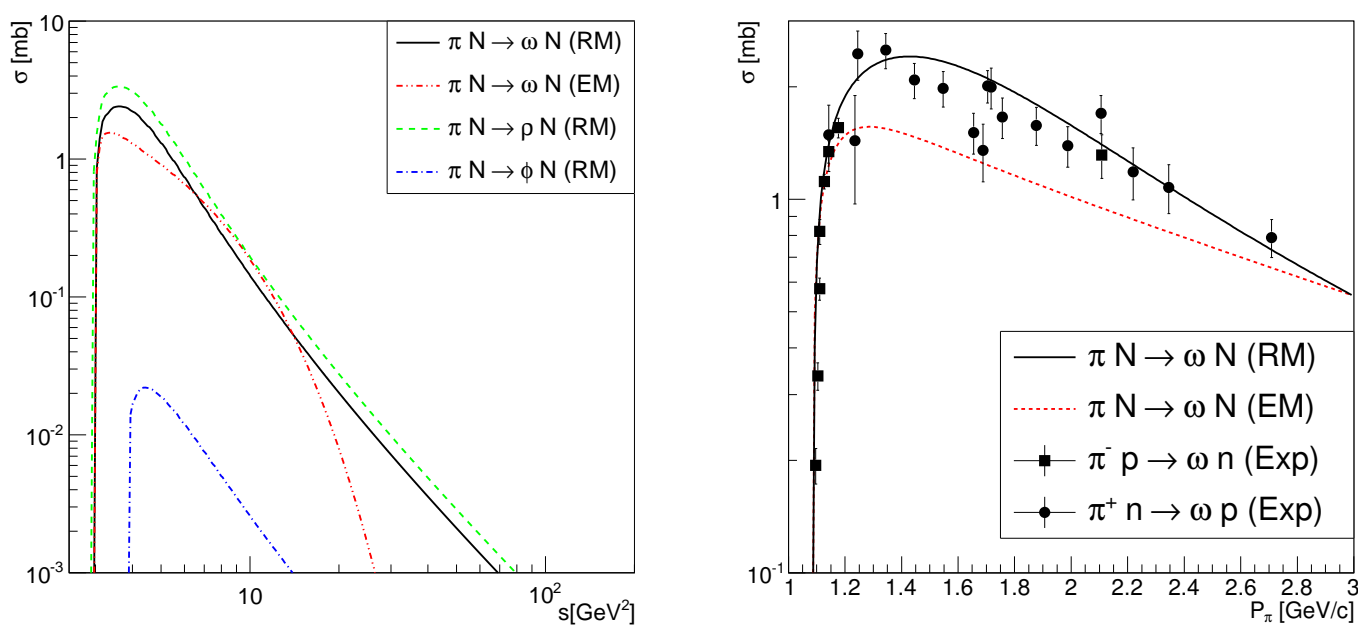

Figura 4.15: Seções de choque totais da interação $\pi N \rightarrow V N$ incluídas no CRISP (esquerda) e comparação da seções de choque totais para a reação $\pi N \rightarrow \omega N$ pelos modelos troca de um méson e ressonante (direita).

comparação, o código CRISP inclui a reação $\omega N \rightarrow \pi N$ calculada usando o modelo ressonante. 


\section{Capítulo 5}

\section{Resultados}

Neste capítulo são apresentados os principais resultados obtidos até o momento que demostram o potencial do modelo desenvolvido neste trabalho. Nesta etapa os resultados são resumidos ao estudo de fenômenos relacionados com a produção dos mésons vetoriais induzidos por fótons e a interação no estado final destes mésons com o núcleo. A principal novidade neste modelo é o estudo de processo nucleares que incluem efeitos coletivos como o bloqueio de Pauli, o movimento fermiônico etc, a partir da inclusão no código de interações binárias entre partículas. É importante assinalar que este trabalho não tem como objetivo principal um estudo aprofundado dos temas expostos a continuação e sim demostrar a efetividade da expansão do código para tratar-los. Os principais resultados são:

1. A dependência da seçao de fotoprodução dos mésons com o número de massa A.

2. O estudo da seção de choque de fotoprodução de mésons do nucleon ligado em função da energia.

3. O estudo do processo de fotoprodução do mésons em energias abaixo do limiar.

4. O estudo do decaimento do estado ligado $J / \Psi$-Núcleo.

5. O espectro de massa do méson $\rho$ gerado no processo de fotoprodução.

6. A transparência nuclear do méson $\omega$.

Os resultados mostrados neste capítulo estão resumidos no artigo intitulado "Nuclear photoproduction of vector mesons within a Monte Carlo approach", publicado no jornal Physical Review C, volumem 89, página 054613 em Maio do 2014 [183]. 


\subsection{Fotoprodução de mésons vetoriais. Seção de choque de fotoprodução vs número de massa}

O processo de fotoprodução dos méson $\rho, \omega$ e $\phi$ foi calculado segundo o modelo CRISP para uma energia $E_{\gamma}=9 \mathrm{GeV}$ nos núcleos ${ }^{12} \mathrm{C},{ }^{27} \mathrm{Al},{ }^{40} \mathrm{Ca},{ }^{56} \mathrm{Fe},{ }^{63} \mathrm{Cu},{ }^{107} \mathrm{Ag},{ }^{138} \mathrm{Ba}$, ${ }^{153} \mathrm{Eu},{ }^{166} \mathrm{Er},{ }^{180} \mathrm{~W},{ }^{197} \mathrm{Au},{ }^{208} \mathrm{~Pb}$ e ${ }^{232} \mathrm{Th}$. Foram simulados $10^{5}$ eventos, resultando em yields de $\sim 10^{4}, \sim 10^{3}$ e $\sim 3 \times 10^{2}$ para $\rho_{0}, \omega$ e $\phi$ respectivamente que correspondem a erros estatísticos de $\sim 1 \%, \sim 3 \%$ e $\sim 6 \%$ respectivamente.

A Figura 5.1 apresenta a razão entre a seção de choque de fotoprodução nuclear dividida e a seção de choque de fotoprodução do próton para vários núcleos. Esta razão é comparada com dados experimentais da Ref. [184] e com o resultado obtido se a seção de choque de fotoprodução crescesse linearmente com o número atômico (linha tracejada) o que indicaria a inexistência do efeito de sombreamento (shadowing). Pode-se observar que nosso resultado está de acordo com os dados experimentais e mostram uma notável diferença da dependência linear. Este comportamento da seção de choque de fotoprodução é uma consequência direta da dominância vetorial nas interações nucleares induzidas por fótons. Devido a isto, a seção de choque de fotoprodução não é proporcional ao número de massa $A$ e sim a uma potência deste $A^{\alpha}$ :

$$
\sigma_{V A}(A)=\sigma_{0} A^{\alpha}
$$

onde $\sigma_{0}$ e $\alpha$ são parâmetros livres. O termo $A_{\text {eff }}=A^{\alpha}$ na equação 5.1 é conhecido como número de massa efetivo que participa na absorção do fóton no núcleo enquanto o parâmetro $\sigma_{0}$ na equação 5.1 é normalmente considerada como a seção de choque de fotoprodução do nucleon ligado. Assim a seção de choque de fotoprodução nuclear pode ser fatorizada em dois termos, $A_{e f f}$ que contêm as propriedades nucleares ou coletivas e $\sigma_{0}$ que inclui as contribuições individuais dos nucleons que formam o núcleo. Esta última é considerada similar para os prótons e nêutrons do núcleo, desde que na energia do fóton estudada, a seção de fotoprodução em prótons e nêutrons livres sejam aproximadamente a mesma.

A partir do comportamento da seção de fotoprodução dos mésons $\rho, \omega$ e $\phi$ em função do número de massa obtida foi calculado o valor do expoente $\alpha$ ajustando os dados à Equação 5.1. O melhor ajuste é mostrado na tabela 5.1 para cada méson estudado. Pode-se observar que os valores do expoente $\alpha$ são muito parecidos para todos os mésons, porém não iguais. Isto pode ser interpretado como o fenômeno de saturação do efeito de sombreamento desde que a energia do processo de fotoprodução estudada esteja longe do limiar dos mésons $\rho, \omega$ e $\phi$. Apesar de obter valores similares do expoente destes mésons, ainda há uma diferença para o valor do méson $J / \Psi$. Isto é devido a que nesta energia 


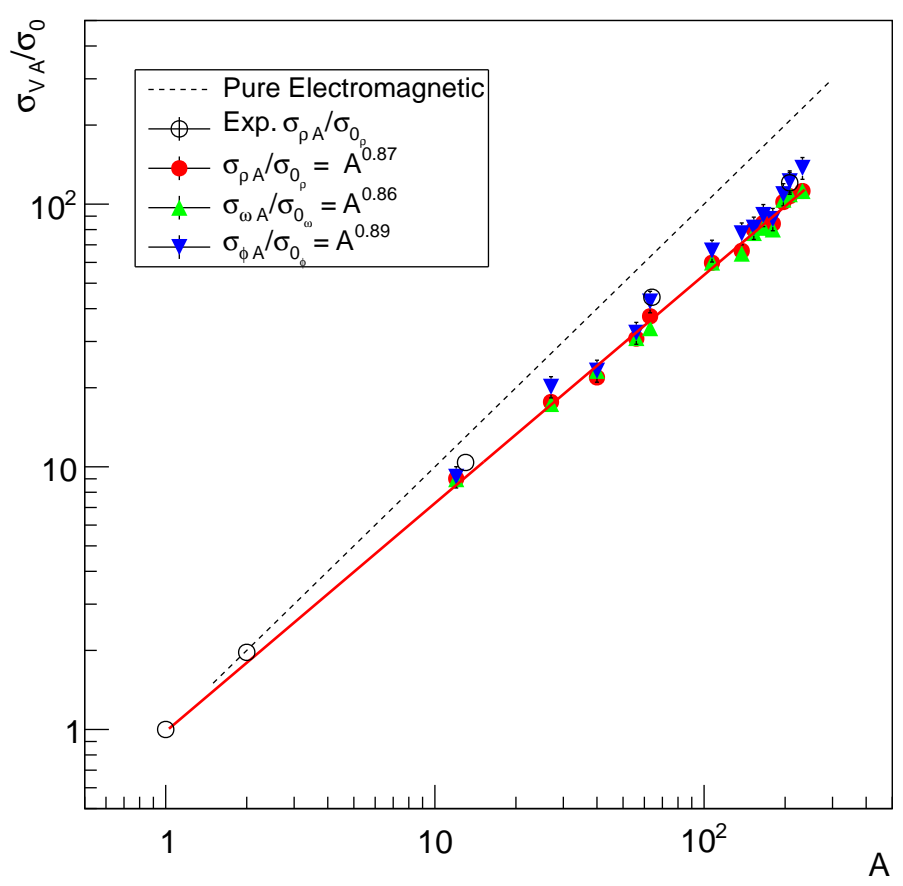

Figura 5.1: Seção de choque de fotoprodução de mésons vetoriais em função do número de massa atômica para uma energia do fóton $E_{\gamma=9 \mathrm{GeV}}$. A figura mostra o ajuste geral para todos os mésons pela Equação 5.1 comparada com dados experimentais da Ref. [184]. O melhor ajuste dos parâmetros é $\sigma_{0}=0.98 \pm 0.03$ e $\alpha=0.87 \pm 0.01$ $\operatorname{com} \chi_{\text {red }}^{2}=3.5$.

o efeito de sombreamento deste último ainda não saturou na energia estudada, como será mostrado mais na frente. Na figura 5.1, representado pela linha cheia vermelha, é mostrado um único ajuste dos dados obtidos com o CRISP para todos os mésons com a equação 5.1. Este ajuste pode ser interpretado como uma média do valor do expoente $\alpha$ de todos os mésons. O expoente obtido neste ajuste é $\alpha=0.87 \pm 0.01$ que está de acordo com o valor apresentado na literatura $\alpha \sim 0.9$ [184].

TABELA 5.1: Valores da seção de choque por nucleon e o expoente $\alpha$ obtido pelo ajuste da seção de choque fotoprodução para vários núcleos com a Equação 5.1.

\begin{tabular}{ccccc}
\hline \hline Meson & $\sigma_{0}[\mathrm{mb}]$ & $\alpha$ & $\sigma_{0_{\text {theo }}}[\mathrm{mb}]$ & $\sigma_{0_{\text {exp }}}[\mathrm{mb}]$ \\
\hline$\rho^{0}$ & $16.8 \pm 0.3$ & $0.87 \pm 0.01$ & 12.6 & $\sim 10.4 \pm 0.7$ \\
$\omega$ & $2.06 \pm 0.10$ & $0.88 \pm 0.01$ & 1.55 & $\sim 1.10 \pm 0.18$ \\
$\phi$ & $0.58 \pm 0.05$ & $0.90 \pm 0.02$ & 0.46 & $\sim 0.55 \pm 0.09$ \\
$J / \Psi$ & $3.41 \mathrm{e}-07 \pm 0.1 \mathrm{e}-07$ & $0.94 \pm 0.02$ & $3.1 \mathrm{e}-07$ & - \\
\hline \hline
\end{tabular}


Na tabela 5.1, é comparada a seção de choque de fotoprodução para o nucleon ligado $\sigma_{0}$ com a seção de choque de fotoprodução do próton teórica $\sigma_{0_{t h e o}}$, dada pelo modelo de dipolo de soft Pomeron, e experimental $\sigma_{0_{e x p}}$, obtidos de vários experimentos. Podese notar que apresentam boa concordância entre eles como é esperado, porém existem diferencias. O efeito de sombreamento observado em nossos cálculos é puramente um efeito nuclear que depende da seção de choque de fotoprodução de mésons vetoriais para prótons e de propriedades do núcleo atômico como raio e densidade. Nota-se que este efeito surge naturalmente no nosso modelo como consequência da inclusão de interação do fóton com o nucleon e da reprodução correta do mecanismo de fotoabsorção. O fato de se obter uma boa descrição deste efeito é uma prova de que a propagação do fóton e sua interação com nucleons é simulada corretamente nos presentes cálculos.

\subsection{Fotoprodução de mésons vetoriais. Seção de choque de fotoprodução do nucleon ligado vs energia}

Nesta seção são mostrados os resultados da seção de choque de fotoprodução do nucleon ligado ( $\sigma_{0}$ da equação 5.1). Esta grandeza é calculada a partir da seção de choque de fotoprodução nuclear normalizada pelo número de massa efetivo $A_{\text {efetivo }}=A^{\alpha}$ calculados na seção anterior e mostrados na tabela 5.1 para cada méson. Para o caso do méson $J / \Psi$ usamos $\alpha=0.94$. Este valor foi obtido a partir de um estudo detalhado do efeito de sombreamento para este méson descrito à frente.

Na figura 5.2 a seção de choque de fotoprodução por nucleon ligado para cada méson vetorial em função da energia do fóton é comparada com dados experimentais obtidos de vários experimentos que foram transformados para o referencial do laboratório. Observa-se uma boa concordância dos nossos resultados com os dados experimentais até algumas centenas de $\mathrm{GeV}$, no entanto, perto do limiar pode-se observar diferenças. Estas discrepâncias no limiar podem estar relacionadas ao fato de que foi usado um valor de $\alpha$ constante. Como discutido adiante (seção 5.3), neste intervalo de energia esta grandeza deve ter alguma dependência com a energia devido à crescente influência de outros efeitos nucleares como por exemplo o bloqueio de Pauli. Este efeito será estudado mais detalhadamente na próxima seção para o méson $J / \Psi$. 


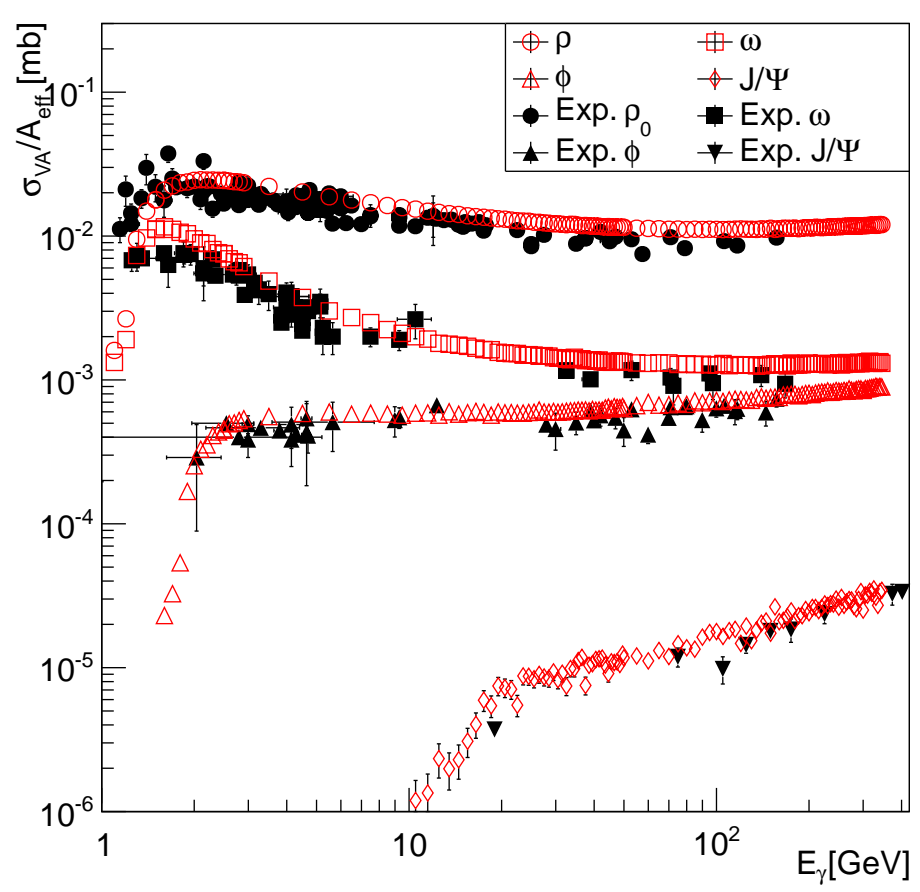

Figura 5.2: Seção de choque de fotoprodução do nucleon ligado vs energia obtido com o código CRISP comparada com dados experimentais.

\subsection{Fotoprodução de mésons vetoriais abaixo do limiar de produção}

O fenômeno da produção de mésons vetoriais induzida por fótons a energias próximas do limiar é um tema de muito interesse já que fornece informações importantes do ambiente nuclear como é o espalhamento $V N \rightarrow V N$ [185]. A amplitude das reações nucleares nestas energias é fortemente modificada por efeitos como o bloqueio de Pauli e o movimento fermiônico. O primeiro modifica fortemente a seção de choque de fotoprodução uma vez que bloqueia colisões que no vácuo seriam permitidas, enquanto que o movimento fermiônico aumenta ou diminui a energia do centro de massa do sistema $\gamma N$ de acordo com o momento do nucleon interagente. Estes efeitos se tornam relevantes em processo em que a energia cinética das partículas geradas em uma colisão é da mesma ordem que a energia cinética do núcleo atômico como acontece justamente perto do limiar. A influência destes fenômenos nucleares na seção de choque de produção de mésons vetoriais irá influenciar diretamente em um comportamento não constante do expoente $\alpha$ da equação 5.1. O cálculo da função $\alpha\left(E_{\gamma}\right)$ é importante para a correta descrição da seção de choque de fotoprodução para os nucleons ligados nas energias perto do limiar. 
Nesta seção é analisado como o movimento fermiônico e o bloqueio de Pauli modificam o processo de fotoprodução e com ele a seção de choque através da função $\alpha\left(E_{\gamma}\right)$.

\subsubsection{Movimento fermiônico}

O movimento fermiônico pode ser interpretado como uma flutuação da energia do fóton incidente no sistema de coordenadas de repouso do nucleon. Uma consequência desta flutuação é que a seção de choque de fotoprodução é maior do que zero abaixo do limiar e diminui menos abruptamente com o decremento da energia como acontece na interação com prótons no vácuo. É importante analisar como o movimento fermiônico modifica a energia do sistema fóton-nucleon e com ela, a seção de choque de fotoabsorção. Seja uma colisão de um fóton entre um nucleon estacionário no sistema de coordenadas do laboratório. A energia ao quadrado do centro de massa $S_{0}$ é:

$$
\begin{aligned}
& p_{\gamma} \equiv\left(E_{\gamma}, 0,0, E_{\gamma}\right), p_{N} \equiv\left(0,0,0, m_{N}\right) \\
& S_{0}=\left(p_{\gamma}+p_{N}\right)^{2}=2 E_{\gamma} m_{N}+m_{N}^{2} .
\end{aligned}
$$

Para uma colisão de um nucleon não estacionário, o movimento fermiônico introduz uma energia extra no sistema $\gamma N$ da forma:

$$
\begin{aligned}
p_{\gamma} & \equiv\left(E_{\gamma}, 0,0, E_{\gamma}\right), p_{N} \equiv\left(p_{x}, p_{y}, p_{z}, E_{N}\right) \\
S & =\left(p_{\gamma}+p_{N}\right)^{2}=2 E_{\gamma} E_{N}+E_{N}^{2}-2 E_{\gamma} p_{x}-\mathbf{p}_{N}^{2} \\
& =2 E_{\gamma} E_{N}+m_{N}^{2}-2 E_{\gamma} p_{x} .
\end{aligned}
$$

As equações 5.3 podem ser reescritas usando a relação $E_{N}=\sqrt{m_{N}^{2}+\mathbf{p}_{N}^{2}}=m_{N}+K_{N}$, onde $K_{N}$ é a energia cinética do nucleon:

$$
S=2 E_{\gamma} m_{N}+m_{N}^{2}+2 E_{\gamma} K_{N}-2 E_{\gamma} p_{x} \equiv S_{0}+\Delta S
$$

onde:

$$
\Delta S=2 E_{\gamma} K_{N}-2 E_{\gamma} p_{x}
$$

é a energia do centro de massa ao quadrado que o sistema fóton-nucleon ganhou pelo movimento fermiônico. A energia do centro de massa atribuída pelo sistema, $\Delta W$, pode ser obtida a partir da definição $W=\sqrt{S} \equiv W_{0}+\Delta W$ onde $W_{0}=\sqrt{S_{0}}$

No código CRISP, o núcleo tem uma estrutura de camadas dos momento lineares dos nucleons de acordo com a seção 2.1.1.1. Lembrando (equação 2.8):

$$
\mathbf{p}^{2}=p_{x}^{2}+p_{y}^{2}+p_{z}^{2}=\left(n_{x}^{2}+n_{y}^{2}+n_{z}^{2}\right)(\Delta p)^{2}=n^{2}(\Delta p)^{2}, n \in \mathbf{N}^{+}
$$


O tamanho da célula $\Delta p$ é obtida de acordo com a energia de Fermi:

$$
E_{F}=\frac{\left(c t e\left[\frac{n_{N}}{A}\right]^{1 / 3}\right)^{2}}{2 m_{N}} \text { e } p_{F}=\sqrt{E_{F}\left(E_{F}+2 m_{e f f}\right)}
$$

onde $m_{e f f}$ é a massa efetiva do nucleon, $n_{N}$ é número de prótons ou nêutrons e $A$ é o número de massa do núcleo. O tamanho da célula é definido como a razão entre o momento de Fermi e o número de camadas ocupadas:

$$
\Delta p=\frac{p_{F}}{n_{o c p}}
$$

onde $n_{\text {ocp }}$ é o número de camadas ocupadas. O efeito do movimento fermiônico pode ser estudado através da flutuação da energia do centro de massa e de laboratório do sistema $\gamma N$ usando as equações prévias. Esta flutuação depende da energia do fóton, do momento do nucleon ou o que é o mesmo, da camada que ocupa e do valor da componente $p_{x}$ do momento. Como exemplo é analisada a flutuação sistema $\gamma N$ para os nucleons do núcleo ${ }^{238} \mathrm{U}$ e energia do fóton igual ao limiar de produção do méson $\mathrm{J} / \Psi E_{0} \sim 8470 \mathrm{MeV}$. Esta energia é suficientemente alta para que o efeito seja perceptível. A flutuação em função da componente do momento $p_{x}$ para algumas camadas é apresentada nas figuras 5.3 nas energias de centro de massa $(W)$ e de laboratório $\left(E_{\gamma}\right)$ para várias camadas do núcleo.

Por exemplo, o núcleo ${ }^{238} \mathrm{U}$ tem 11 camadas completas de nêutrons e 9 de prótons. Na primeira camada $(n=1)$, os nêutrons tem um valor de momento $p_{\text {nêutron }}(n=1)=$ $24.13 \mathrm{MeV}$ enquanto que para os prótons $p_{\text {próton }}(n=1)=25.22 \mathrm{MeV}$. Este valor de momento introduz uma energia máxima (para valor mínimo do momento $p_{x}=-p$ ) no sistema $\gamma N$ de $\Delta W \sim 50 \mathrm{MeV}$ para nêutrons e prótons no sistema de centro de massa. Esta energia, no referencial de laboratório, se traduz na diminuição do limiar de produção em $\Delta E_{\gamma} \sim-200 \mathrm{MeV}$. Na última camada os nêutrons tem um momento $p_{\text {nêutron }}(n=11)=265.46 \mathrm{MeV}$ e os prótons $p_{\text {próton }}(n=9)=226.94 \mathrm{MeV}$. Este valor de momento poderão incrementar o valor da energia do centro de massa do sistema fótonnêutron em $\Delta W \sim 570 \mathrm{MeV}$ e $\Delta E_{\gamma} \sim-2.7 \mathrm{GeV}$ no referencial do laboratório enquanto que para o sistema fóton-próton a energia do centro de massa poderá ser incrementada em $\Delta W \sim 500 \mathrm{MeV}$ e o valor do limiar de produção poderá diminuir em $\Delta E_{\gamma} \sim-2.2$ $\mathrm{GeV}$ (referencial do laboratório).

O processo de produção de mésons vetoriais induzidos por fótons abaixo do limiar poderá ser melhor estudado para processos de fotoprodução com alto valor de energia de limiar, onde o efeito do movimento fermiônico é maior. Assim é proposto o estudo do processo de fotoprodução do méson $J / \Psi$ perto do limiar no núcleo ${ }^{238} \mathrm{U}$ com valor de energia de limiar de produção $W_{0} \sim 4.096 \mathrm{GeV}$ no sistema de centro de massa e $E_{\gamma} \sim 8.47 \mathrm{GeV}$ no 

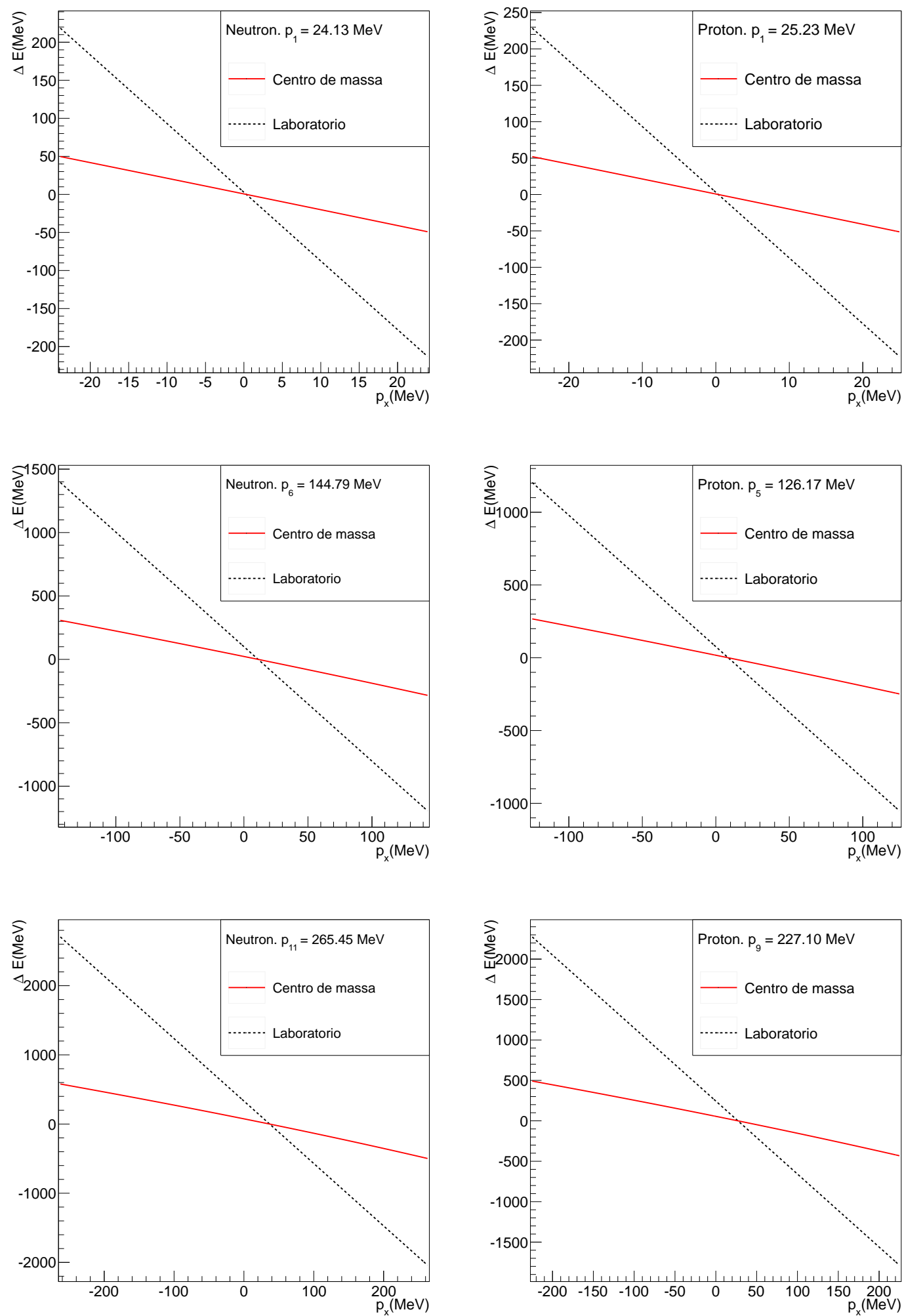

FIGURA 5.3: Flutuação energética dada pelo movimento fermiônico sobre o sistema $\gamma N$ para nêutrons e prótons do núcleo ${ }^{238} \mathrm{U}$ e energia do fóton $\sim 8470 \mathrm{MeV}$ em função do momento no eixo $x, p_{x}$, para diferentes camadas. 
referencial do laboratório. Outro efeito nuclear muito importante nestas energias que se soma ao movimento fermiônico é o mecanismo de bloqueio de Pauli que será analisado na próxima seção.

\subsubsection{Mecanismo de bloqueio de Pauli}

O efeito de sombreamento descrito matematicamente através do expoente $\alpha$ na Equação 5.1 é modificado para energias perto do limiar de produção já que efeitos nucleares como o bloqueio de Pauli se manifestam com maior intensidade. Para estudar a importância deste efeito é conveniente definir o índice de bloqueio $\eta$ :

$$
\eta=\frac{\eta_{b}}{\eta_{c}}
$$

onde $\eta_{b}$ é o número de processos de fotoprodução de mésons que foram bloqueados pelo principio de Pauli e $\eta_{c}$ é o número de mésons vetoriais efetivamente criados. Este expoente pode ser ajustado a uma função exponencial

$$
\eta=\alpha \mathrm{e}^{\beta E_{\gamma}} .
$$

como é mostrado na figura 5.4. Nesta figura é apresentado o comportamento do índice $\eta$ no processo de fotoprodução do méson $J / \Psi$ no núcleo ${ }^{238} \mathrm{U}$ no intervalo de energia que inclui o limiar de produção. Pode-se notar o aumento do índice a medida que a energia do fóton diminui desde um valor $\sim 0$ para $E_{\gamma}=11.5 \mathrm{GeV}$ até $\sim 7$ para $E_{\gamma}=7.5 \mathrm{GeV}$ onde, de acordo à definição, são bloqueados aproximadamente 7 processos por um permitido.

Devido à análise anterior, neste intervalo de energia o número de massa efetiva $A_{\text {eff }}=$ $A^{\alpha}$ deverá aumentar devido ao efeito do bloqueio de Pauli. Longe do limiar o efeito de Pauli é irrelevante e a principal contribuição ao número de massa $A_{\text {eff }}$ e da densidade e do raio do núcleo. Daí foi obtido um valor de expoente $\alpha \sim 0.9$ para os mésons $\rho$, $\omega$ e $\phi$ que pode ser considerado constante. Usando o comportamento do bloqueio de Pauli, pode-se predizer o incremento de $A_{\text {eff }}$ e seu comportamento perto ao limiar de produção.

\subsubsection{Comportamento sub-limiar de $A_{\text {eff }}(E)$}

O comportamento da $A_{\text {eff }}$ para energias próximas ao limiar reflete uma combinação de efeitos como o efeito de sombreamento e o bloqueio de Pauli. Como vimos acima, nas regiões onde existe uma forte dependência da seção de choque com a energia do fóton, o movimento de Fermi dos nucleons também modificam o $A_{e f f}$. Para um estudo 


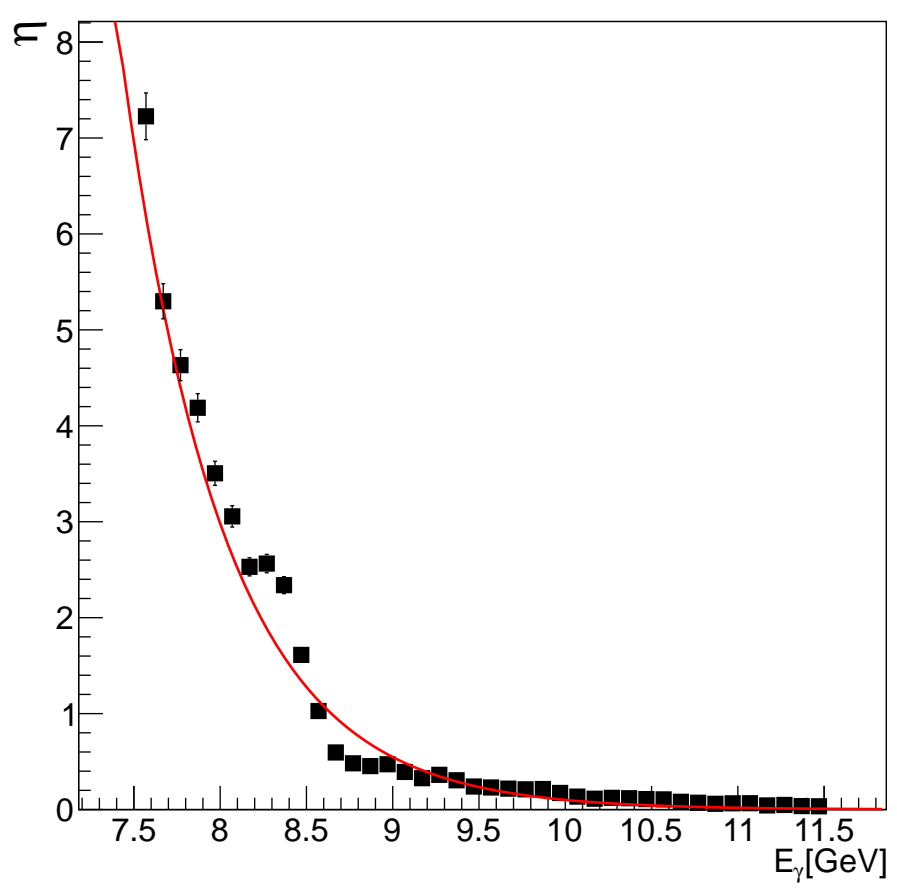

Figura 5.4: Efeitos do bloqueio de Pauli na fotoprodução do méson $J / \Psi$ perto ao limiar. Os parâmetros obtidos do melhor ajuste dos dados à equação 5.8 são $\alpha=$ $2.3 \cdot 10^{6} \pm 3.5 \cdot 10^{5}$ e $\beta=-1.7 \pm 0.02$ com $\chi_{\text {red }}^{2}=5.5$.

quantitativo deste efeito deverá ser estudado o comportamento desta grandeza. Para isso, foi feita a mesma análise para várias energias apresentado na seção 5.1 onde a seção de choque em função do número de massa $A$ foi ajustado a equação 5.1

$$
\sigma_{V A}(A)=\sigma_{0} A^{\alpha}
$$

A seção de choque de fotoprodução de $J / \Psi$ é calculada nos núcleos ${ }^{40} \mathrm{Ca},{ }^{63} \mathrm{Cu}{ }^{107} \mathrm{Ag}$, ${ }^{138} \mathrm{Ba},{ }^{166} \mathrm{Er},{ }^{180} \mathrm{~W},{ }^{208} \mathrm{~Pb} \mathrm{e}{ }^{238} \mathrm{U}$ para energias no intervalo de energia $7 \leq E_{\gamma} \leq 14 \mathrm{GeV}$, que inclui o limiar de fotoprodução deste méson $\sim 8.47 \mathrm{GeV}$. Para cada uma delas, os valores de seção de choque obtida em cada núcleo foram ajustados pela equação 5.1 usando $\sigma_{0}$ e $\alpha$ como parâmetros livres. O yield obtido para o méson $J / \Psi$ em cada energia foi fixado em $10^{3}$, o que implica em um error estatístico de $\sim 3 \%$.

Para realizar esta análise foram usadas técnicas de redução de variância com o objetivo de aumentar a estatística. A técnica está baseada em incrementar a taxa de produção do méson $J / \Psi$ sem variar a seção de choque total, desta forma o efeito de sombreamento não é alterado. O incremento da seção de choque de produção para o nucleon $\sigma_{0} \mathrm{em}$ função da energia pode ser observado ver nos ajustes realizados, no entanto o parâmetro carece de sentido real. A possibilidade de usar este tipo de técnicas no código CRISP faz 
deste uma ferramenta poderosa para o estudo de fenômenos com baixa probabilidade de acontecer.

Como é esperado, o expoente $\alpha$ varia com a energia do fóton. Nas figuras 5.5 são mostrados alguns resultados para a seção de choque de fotoprodução em função do número de massa junto com o melhor ajuste dos parâmetros $\sigma_{0}$ e $\alpha$ a função 5.1 para várias energias. $\mathrm{O}$ conjunto de valores de $\alpha$ obtidos em cada ajuste para cada energia é mostrado na figura 5.6. Pode-se notar o decrescimento do expoente $\alpha$ com o aumento da energia até que esse atinja um valor constante. Estes valores de $\alpha$ em função da energia ajustam bem a equação:

$$
\alpha\left(E_{\gamma}\right)=\alpha_{0}+e^{\beta\left(E_{\gamma}-E_{\gamma_{0}}\right)} .
$$

O melhor ajuste dos valores do expoente a equação 5.9 resulta na obtenção dos parâmetros $\alpha_{0}=0.94$ e $E_{0}=7.1 \mathrm{GeV}$. O primeiro pode ser interpretado como o limite da função $\alpha\left(E_{\gamma}\right)$ para energias suficientemente acima do limiar, onde pode ser considerado irrelevante o bloqueio de Pauli. Este exponente foi o valor usado a priori no cálculo da seção de choque de fotoprodução por nucleon ligado na seção 5.2. É importante assinalar que $\alpha_{0}=0.94$ não corresponde ao efeito de sombreamento saturado. De acordo a equação 2.47

$$
\Delta l=\frac{2 \hbar c E_{\gamma}}{\mu^{2}},
$$

para o valor da massa $\mu_{J / \Psi} \sim 3.1$, o efeito de sombreamento para o méson $J / \Psi$ saturará para um valor da energia $E_{\gamma} \sim 30 \mathrm{GeV}$ que é quando o valor do comprimento do estado hadrônico do fóton dado por Eq. 2.47 for da ordem do raio nuclear. O segundo parâmetro, $E_{0}=7.1 \mathrm{GeV}$, corresponde a um novo limiar no referencial de laboratório. Abaixo desta energia o processo de fotoprodução pode realmente acontecer de acordo com o efeito do movimento fermiônico que consegue diminuir o limiar de produção até $E_{0} \sim 6 \mathrm{GeV}(\Delta E \sim-2.5 \mathrm{GeV})$, mas o bloqueio de Pauli é tão intenso que não permite nenhuma colisão.

Obtém-se assim uma boa descrição do comportamento $\alpha\left(E_{\gamma}\right)$ para o méson $J / \Psi$ que permite a correta descrição do processo de fotoprodução sub-limiar deste méson como é discutido na próxima seção. É importante notar dois fatos: 1) que o ajuste resultou em um comportamento exponencial similar ao obtido na descrição do bloqueio de Pauli e 2) que três mecanismos se combinam aqui para produzir o comportamento de $\alpha\left(E_{\gamma}\right)$ : o mecanismo do bloqueio de Pauli, o movimento fermiônico e o efeito de sombreamento. Esta combinação de efeitos é complexa e devido a isso não se dará nenhum significado físico a este expoente. 

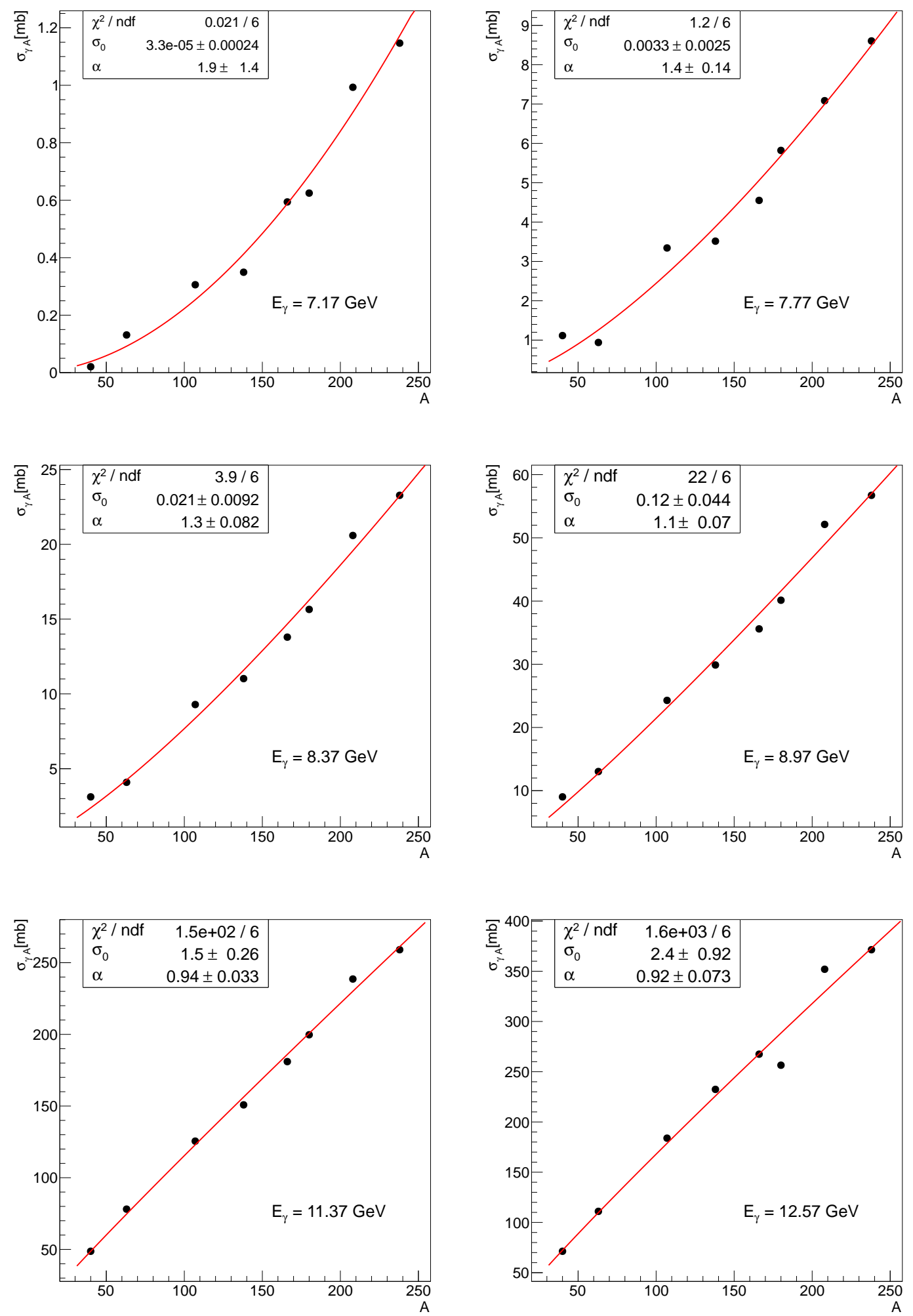

FIGURA 5.5: Seção de choque de fotoprodução de méson vetoriais em função do número de massa para varias energias do fóton perto ao limiar. 


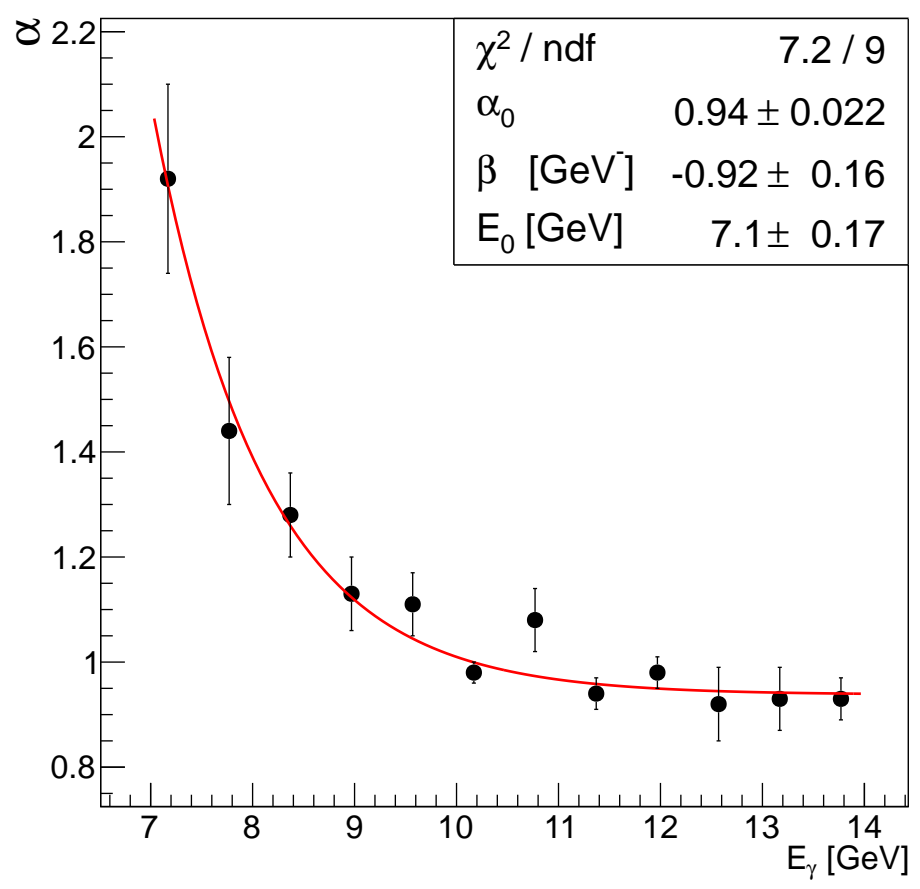

Figura 5.6: Comportamento do expoente $\alpha$ na fotoprodução do méson $J / \Psi$ perto ao limiar ajustado à equação 5.9 .

\subsubsection{Fotoprodução do méson $\mathrm{J} / \Psi$ perto ao limiar}

Uma vez obtido o comportamento do expoente $\alpha\left(E_{\gamma}\right)$, é possível calcular a seção de choque de fotoprodução perto do limiar usando um número de massa mais realista $A_{e f f}=A^{\alpha}$. Todos os efeitos nucleares estão incluídos neste novo $A_{\text {eff }}$. Usando a equação 5.9 o novo $A_{e f f}$ é determinado como:

$$
A_{e f f}=A^{\alpha_{0}+e^{\beta\left(E \gamma-E \gamma_{0}\right)}}
$$

onde, para o méson $J / \Psi, \alpha_{0} \sim 0.94$, o valor do coeficiente ângular é $\beta \sim-0.92 \mathrm{GeV}^{-} \mathrm{e}$ o limiar de produção no referencial de laboratório é $E_{\gamma_{0}} \sim 7.1 \mathrm{GeV}$.

O estudo da seção de choque de fotoprodução por nucleon ligado para o méson $J / \Psi$ obtida no processo de produção de mésons vetoriais no núcleo ${ }^{12} \mathrm{C}$ é apresentado na figura 5.7, comparada com a seção de choque de fotoprodução do méson $J / \Psi$ no próton, calculada usando o modelo de soft pomeron. A seção de choque obtida de acordo com equação 5.1, é mostrada usando duas normalizações diferentes relacionadas com o expoente. Em um caso (quadrados vermelhos) é usado o valor do expoente saturado $\alpha=0.94$ enquanto que no outro caso (círculos pretos) o expoente inclui a dependência com a energia $\alpha\left(E_{\gamma}\right)$ (equação 5.9) já que perto do limiar o efeito de sombreamento ainda 
não está saturado. Em ambos resultados pode ser observada uma significativa produção

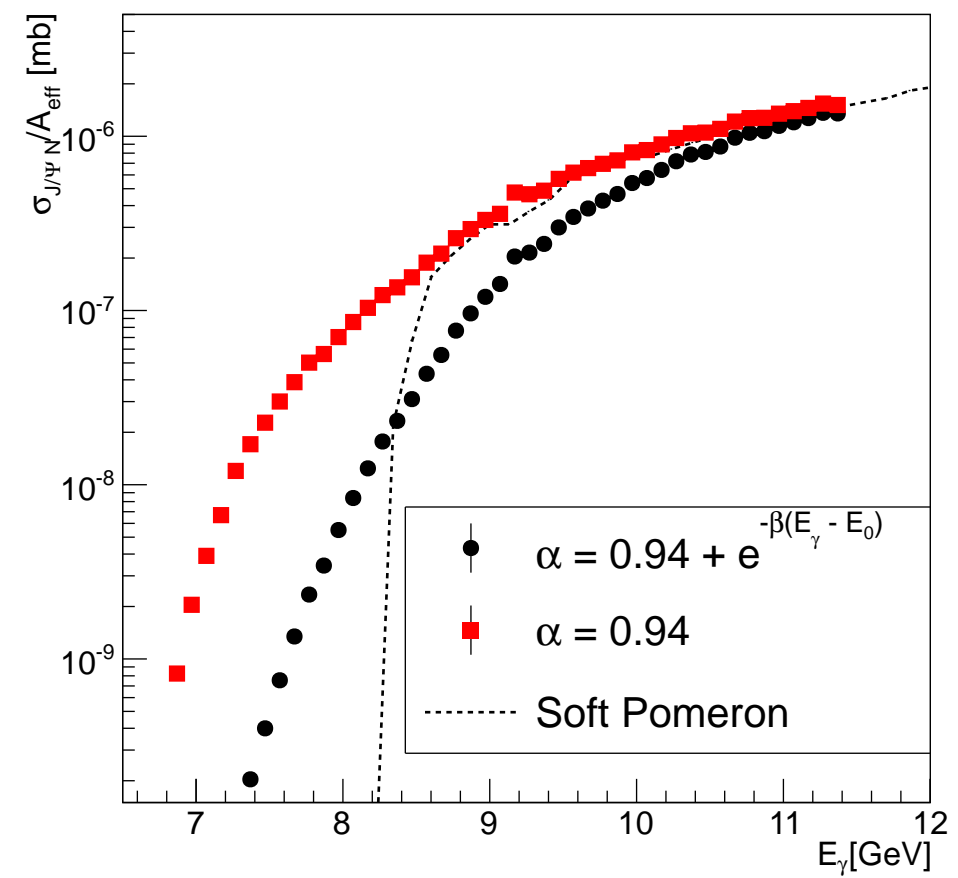

Figura 5.7: Seção de choque por nucleon ligado do méson $J / \Psi$ perto ao limiar usando duas normalizações: com um efeito de sombreamento saturado e (quadros vermelhos) e com um efeito de sombreamento não saturado dependente da energia (círculos pretos).

de mésons $J / \Psi$ abaixo do limiar do produção no próton. Esta é uma consequência do movimento fermiônico dos nucleons dentro do núcleo que produz uma flutuação da energia do fóton $\Delta E_{\gamma} \sim 2.7 \mathrm{GeV}$ que é a causa que estas seções de choque de produção do méson crescer mais suavemente no ambiente nuclear do que no vácuo. A diferença entre elas é o efeito do mecanismo de Pauli que produz uma abrupta queda da seção de choque de fotoprodução quando esse é considerado.

\subsection{Largura do nucleo composto $V-A$}

O modelo CRISP permite o estudo não só da interação das partículas no meio nuclear, mas também dos efeitos dinâmicos na largura do sistema composto formado pelo méson e o núcleo. Este tema apresenta um crescente interesse na comunidade científica pela possibilidade do estudo das forças de Van der Waals de cor na QCD [186] no núcleo atômico e o possível deslocamento negativo da largura da massa do méson produzido ligado ao núcleo [187-189]. 
Aqui é apresentado um efeito puramente nuclear que dever ser considerado nos cálculos em QCD e mostra como o código CRISP pode ser usado para o estudo das propriedades do méson ligado $J / \Psi$. É estudado o tempo de vida de $J / \Psi$ no núcleo ${ }^{238} \mathrm{U}$, mostrado na figura 5.8, através de dois tipos de decaimento do sistema méson $J / \Psi$-núcleo: pela dissociação do méson na interação com nucleons e pelo processo de emissão do núcleo. Com o código CRISP pode-se estudar o comportamento de qualquer partícula ao longo do tempo, e assim diferenciar se esta é emitida ou dissociada pela interação com um nucleon. É possível analisar ambos os decaimentos pelo estudo do processo de fotoprodução nuclear do méson $J / \Psi$ com e sem interação no estado final já que com FSI os dois tipos de decaimento estão incluídos enquanto que sem FSI é só o processo de emissão.

Na figura é mostrado o tempo médio total com FSI (quadrados pretos) que inclui os dois decaimentos. Eles são diferenciados em círculos e quadrados abertos. Ao mesmo tempo é mostrado o tempo de dissociação pelo processo de emissão para a fotoprodução nuclear sem FSI que coincide com o tempo de dissociação total (círculos vermelhos). Este processo é visto na escala mostrada no eixo da direita do gráfico.

É observado que o tempo médio do decaimento do méson ligado $J / \Psi$ pela interação com os nucleons é quase idêntico ao tempo de vida médio total para o processo de fotoprodução com FSI. Isto significa que a dissociação é o processo dominante no decaimento do sistema $J / \Psi$-núcleo enquanto a emissão de $J / \Psi$ corresponde a $\sim 2 \%$ de decaimento, o que é verificado na contagem dos números de mésons que são efetivamente emitidos fora do núcleo. O tempo de vida médio do processo de emissão pode ser calculado com e sem FSI, assim pode-se ver que este se incrementa $\sim 0.2 \mathrm{fm}$ até $\sim 6 \mathrm{fm}$ respectivamente. Este último valor corresponde aproximadamente ao raio do núcleo, assim, a emissão de mésons para $t=\sim 0.2 \mathrm{fm}$ corresponde um sistema $J / \Psi$-núcleo no qual o méson é produzido perto da superfície nuclear.

\subsection{O espectro de massa do méson $\rho$}

De acordo com o modelo VMD a componente hadrônica do fóton interage com o nucleon formando o sistema $\left\langle(q \bar{q})^{J=1} \mid N\right\rangle$. A massa do méson que se forma tem uma distribuição para conservar a energia deste sistema. O espectro de massa dos mésons gerados no meio nuclear é uma questão importante da física nuclear ois ajuda na interpretação dos fenômenos com interação forte no regime não perturbativo da QCD [190, 191]. A forma funcional exata para o espectro de massa, que é obtida pelo cálculo da seção de choque 


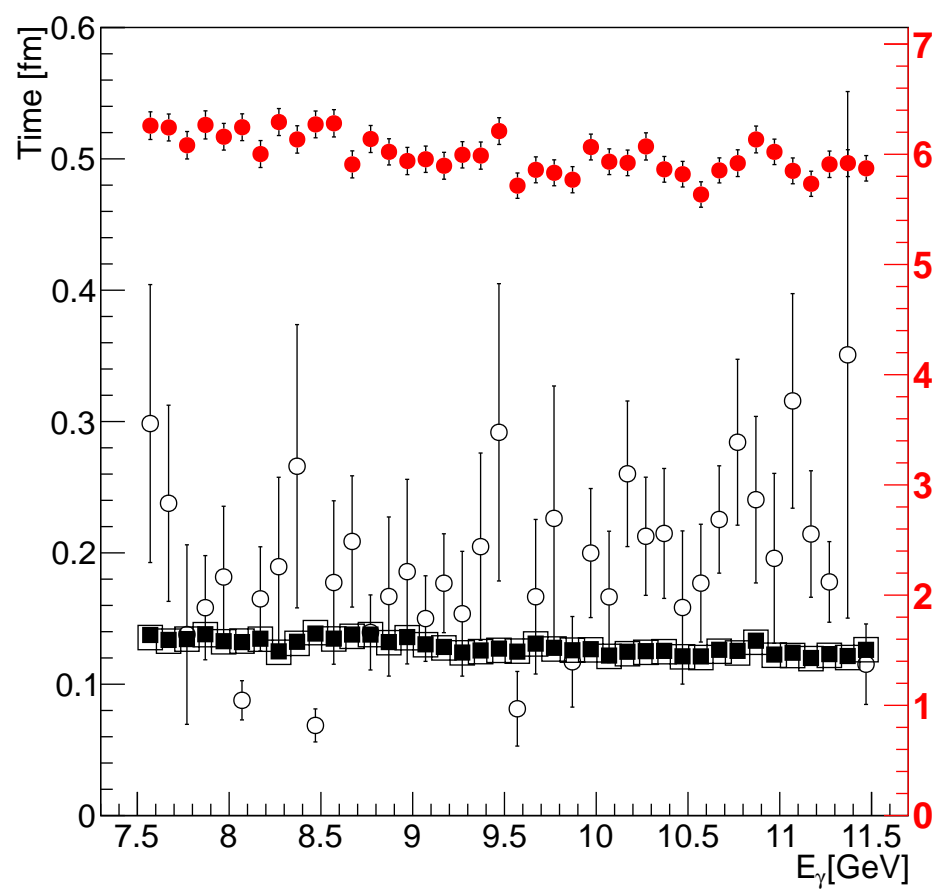

Figura 5.8: Tempo de dissociação do méson $J / \Psi$ com (quadrados pretos) e sem FSI (círculos vermelhos) na escala da direita. O tempo médio de dissociação com FSI é dado por dois processos mostrados na figura: a emissão do méson fora do núcleo (círculos abertos) e a interação com o nucleons (quadrados abertos).

de produção incluindo a largura de decaimento leptônico do méson é dada por [190]:

$$
A(\mu)=\frac{2}{\pi} \frac{\mu^{2} \Gamma(\mu)}{\left(\mu^{2}-M_{\rho}^{2}\right)^{2}+\mu^{2} \Gamma^{2}(\mu)}
$$

onde $\Gamma(\mu)$ é a largura da ressonância, $M_{\rho}$ é a massa do polo $\rho$ e $\mu$ é a massa invariante do par $e^{+} e^{-}$. A equação 5.11 pode ser substituída como boa aproximação, por uma distribuição de Breit-Wigner dividida por um fator $\mu^{3}$, já que o modelo de dominância vetorial propõe que o propagador do fóton é da forma $1 / q^{2}=1 / \mu^{2}$ multiplicando a amplitude por um fator $1 / \mu^{4}$ enquanto que a contribuição do espaço de fase é um fator $\mu$.

O efeito quântico da distribuição de massa dos méson é simulado no código CRISP gerando a massa a partir de uma distribuição de Breit-Wigner com largura definida pelo tempo de vida médio do méson e adicionando a proibição energética de que a massa deste não pode ser maior do que a energia disponível para a criação do méson, isto é,

$$
m_{V}<\sqrt{s}-m_{N}
$$




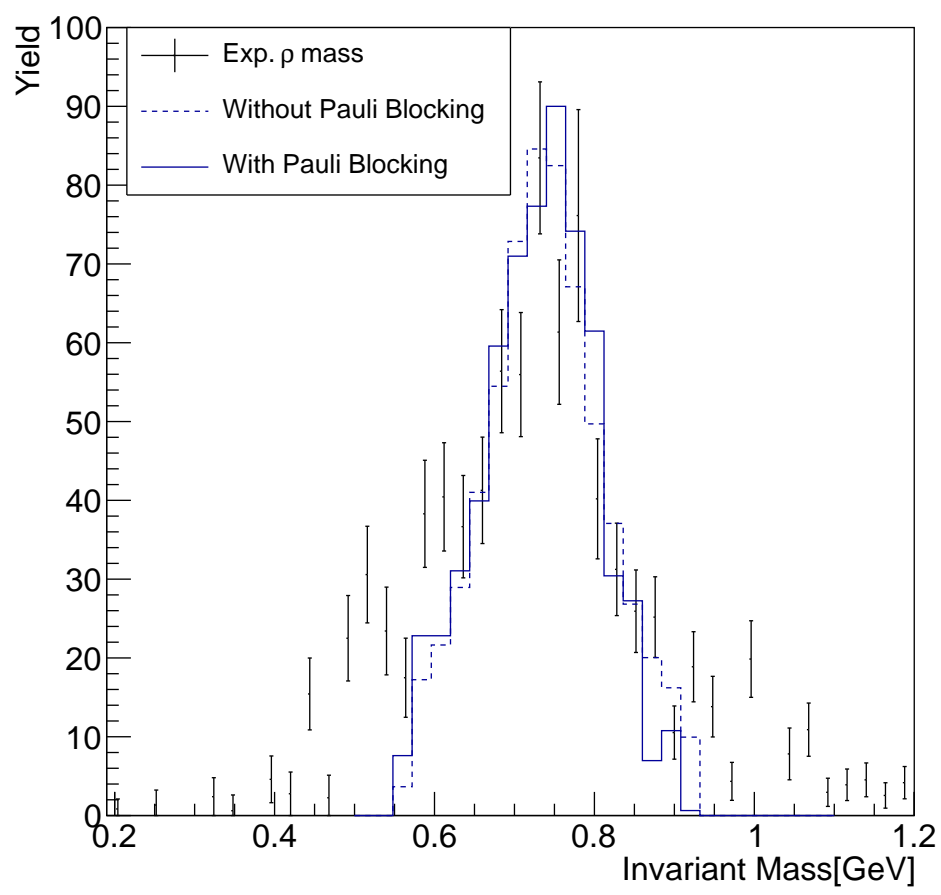

Figura 5.9: Distribuição da massa invariante do méson $\rho$ gerado no processo de fotoprodução no ${ }^{56} \mathrm{Fe}$ com o código CRISP comparada com dados experimentais da Ref. [191].

onde $m_{N}$ é a massa do nucleon. No meio nuclear outra modificação se sobrepõe a esta, o mecanismo de bloqueio de Pauli.

Na figura 5.9 é apresentada a distribuição de massa gerada no processo de fotoprodução com fótons com energia $E_{\gamma}=1.1 \mathrm{GeV}$ para o ${ }^{56} \mathrm{Fe}$. Nesta figura as linhas pontilhadas se referem a uma distribuição de massa no vácuo e as linhas cheias mostram o espectro de massa do méson gerado sob a influência do ambiente nuclear. Estas distribuições são comparadas com os dados experimentais da Ref. [191]. O resultado mostra o efeito nuclear no deslocamento das massas invariantes às menores energias da distribuição de Breit-Wigner.

O espectro obtido para o méson $\rho$ com o CRISP não é simétrico. Ele apresenta um crescimento do yield com o aumento da massa até $\sim 0.75 \mathrm{GeV}$, a massa do méson $\rho$. Acima deste valor o yield experimenta uma queda menos suave que o incremento abaixo deste valor. Este comportamento é devido ao mecanismo de bloqueio de Pauli na interação do estado final do nucleon resultante já que um maior valor da massa do méson gerado no processo de fotoprodução resultará na produção de um nucleon com menor valor de momento e assim um efeito de bloqueio de Pauli mais forte sobre este. Este efeito pode ser visto pela comparação do espectro obtido com o código CRISP com 
(linha cheia) e sem (linha pontilhada) bloqueio de Pauli da figura 5.9. Sem bloqueio o espectro é aproximadamente simétrico enquanto que sua inclusão faz o espectro de massa se aproximar da forma do funcional 5.11.

\subsection{Transparência do méson $\omega$}

Ao longo deste trabalho é mostrado que a FSI dos mésons produzidos nas reações fotonucleares usando o modelo CRISP inclui vários fenômenos. Ao nível individual inclui as interação dos mésons com núcleons de acordo com os canais adicionados e descritos na seção 4.2, entretanto, ao nível coletivo estão incluídos o efeito de sombreamento, o bloqueio de Pauli, o movimento fermiônico e a distribuição de massa do méson gerada na fotoprodução e no ambiente nuclear. Devido à alta complexidade das fotorreações, é usual estudar estes processos por grandezas que tratam o núcleo como uma "caixa preta". Uma destas grandezas usadas para o estudo da interação no estado final das partículas no meio nuclear é a transparência. O conceito intuitivo de "transparência" pode ser analisado nas figuras 4.13 onde os mésons $\pi, \rho$ e $\omega$ apresentam uma grande seção de choque de interação com o nucleon, assim o núcleo é menos "transparente" para esses mésons. Este método foi aplicado em vários trabalhos envolvendo os mésons $\omega$ e $\phi$ [192-197], uma vez que a grande largura do méson $\rho$ faz com que decaia sempre dentro do núcleo. A transparência é definida como a razão entre a seção de choque nuclear e $A$ vezes a seção de choque por nucleon do processo inclusivo de fotoprodução de $\omega$, ou o que é o mesmo

$$
T=\frac{\sigma_{\gamma A \rightarrow V X}}{A \sigma_{\gamma N \rightarrow V X}} .
$$

$T$ descreve a atenuação do fluxo de mésons $\omega$ no meio nuclear e está associado à parte absortiva do potencial nuclear do méson $\omega$, e com ele, a sua largura dos canais inelásticos no meio nuclear. Usualmente, a transparência usada para a análise de diferentes modelos e dados experimentais é uma transparência normalizada aos dados do núcleo ${ }^{12} \mathrm{C}$ i.e.,

$$
T_{A}=\frac{12 \sigma_{\gamma A \rightarrow V X}}{A \sigma_{\gamma}{ }^{12} C \rightarrow V X}
$$

Na figura 5.10 é mostrado o cálculo da transparência normalizada com relação ao carbono obtida com o código CRISP comparada com os dados experimentais [192]. Estes foram obtidos no experimento de produção de mésons realizado no Crystal Barrel detector no ELSA, Bonn, onde as fotorreações são induzidas por Bremsstrahlung. A transparência foi calculada através do modelo CRISP, para o processo de fotoprodução para uma energia fixa no referencial de laboratório $E_{\gamma}=1.7 \mathrm{GeV}$ que é a energia média entre os valores do fóton do experimento no ELSA $1.2 \mathrm{GeV} \leq E_{\gamma_{\exp }} \leq 2.2 \mathrm{GeV}$. Esta 

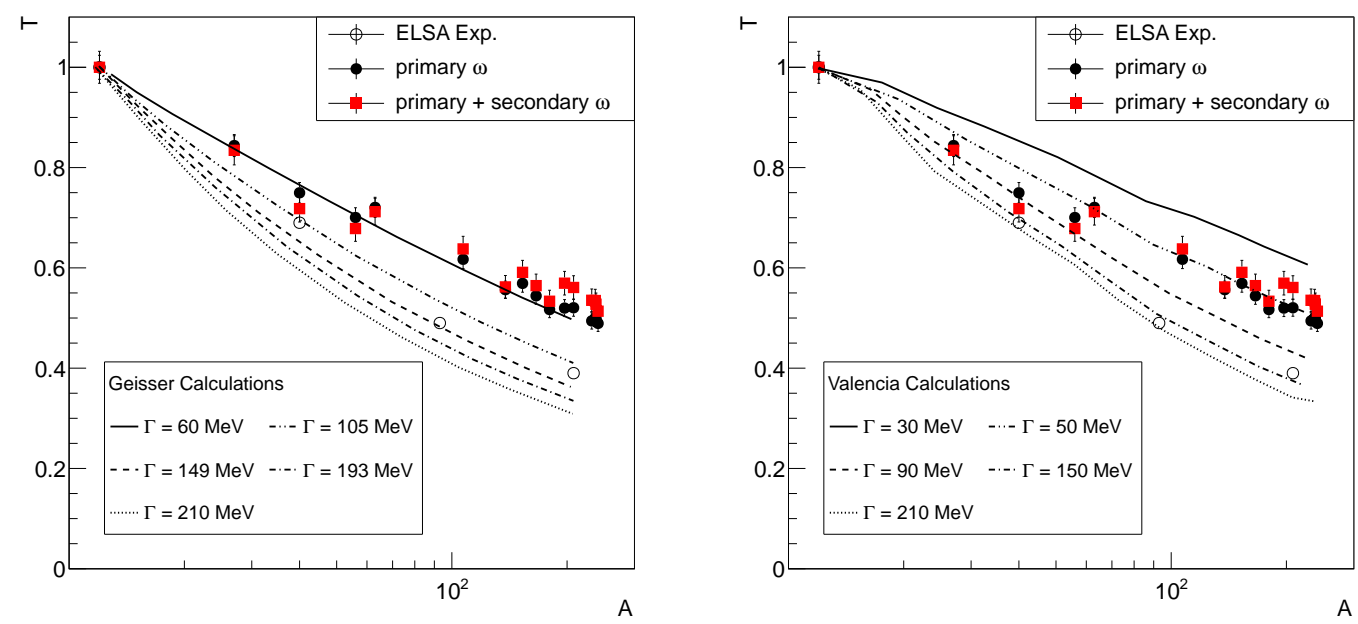

Figura 5.10: Transparência do méson $\omega$ obtida com o código CRISP para $E_{\gamma}=$ $1.7 \mathrm{GeV}$.

aproximação está baseada no fato de que os valores de transparência obtidos neste experimento são integrados em todos os valores de momento do méson $\omega$ detectados [192].

Na figura 5.10, os resultados são comparados com duas abordagens teóricas do problema: um estudo realizado pelo grupo de Geissen usando o modelo de transporte Boltzmann-Uehling-Uhlenbeck [198] figura 5.10 (esquerda) e uma análise por Monte Carlo realizada pelo grupo de Valencia [199] figura 5.10 (direita). Os dados destas abordagens foram obtidos da referência [192]. É observada uma boa concordância com os dados experimentais para núcleos leves até $A \sim 50$, não acontecendo assim para núcleos mais pesados. O resultado pode estar relacionado com a produção do méson $\omega$ na interação do estado final dos outros mésons que será mais relevante em núcleos pesados, ou seja, a produção secundária de mésons induzidos por outros mésons como são $\pi$ e $\rho$.

O modelo CRISP possibilita "ligar" e "desligar" canais de interação, modificando a FSI e assim conseguir um estudo aprofundado dos processos. Esta potencialidade do código é aplicado neste estudo como é mostrado na figura 5.10 onde é apresentado o cálculo da transparência com e sem a inclusão da produção secundária de mésons $\omega$ pela processo FSI. A transparência sem levar em conta a produção secundária é um poco menor do que no cálculo incluindo esta, mas ainda está acima do que os dados experimentais. Isto indica que algum outro processo importante não está sendo levado em conta. Nós continuamos o estudo desta grandeza na atualidade com o objetivo de melhorar este resultado. 


\section{Capítulo 6}

\section{Conclusões e próximos passos}

Vários mecanismos de produção de mésons vetoriais e de sua interação com a matéria nuclear foram incluídos no código CRISP com o objetivo de expandir o intervalo de simulação deste até as altas energias. O processo de fotoprodução de mésons vetoriais é tratado na abordagem do modelo de dominância vetorial, enquanto a interação dos mésons no meio nuclear é tratado, como primeira aproximação, como colisões binárias entre partículas. Foram assim incluídos vários canais de interação envolvendo fótons, núcleons e mésons vetoriais reportados na literatura e usando vários modelos como o modelo de soft Pomeron, o modelo de troca de um méson e o modelo ressonante. Neste ponto o código CRISP consegue estudar reações nucleares induzidas por fótons uma vez que as colisões incluídas por estes modelos se somam aos efeitos nucleares do modelo do código CRISP como o movimento fermiônico, o bloqueio de Pauli, as emissões préequilíbrio etc.

Foram introduzidos os mésons $\rho, \omega, \phi$ e $J / \Psi$ e suas interações com o meio nuclear. Foi preciso uma exaustiva revisão bibliográfica para encontrar os modelos que conseguissem reproduzir satisfatoriamente os dados experimentais da fotoprodução de mésons vetoriais, a interação com os nucleons e ao mesmo tempo, se ajustassem às necessidades computacionais do código CRISP, que se concentra na eficiência da simulação. Do ponto de vista computacional o código CRISP foi paralelizado incrementando o número de eventos por tempo de simulação e diminuindo em grande medida o erro estatístico.

O trabalho foi desenvolvido cumprindo os objetivos propostos no capítulo 1, assim:

- Foram implementados os canais de fotoprodução dos mésons vetoriais $\rho, \omega, \phi$ e $J / \Psi$ no nucleon como mecanismos iniciais que inicializam a cascata intranuclear. Para a descrição das fotorreações foi escolhido o modelo de dipolo de soft Pomeron proposto por E. Martynov, E. Predazzi e A. Prokudin o qual reproduz corretamente 
os dados experimentais da seção de choque desde o limiar até as altas energias para fótons reais e virtuais com valor da virtualidade $0 \leq Q^{2} \leq 35 \mathrm{GeV}^{2}$, para um valor de momento transferido $0 \leq|t| \leq 1.6 \mathrm{GeV}^{2}$. Este é um modelo que inclui um total de 12 parâmetros ajustados a um total de 357 pontos experimentais de seção de choque total e diferencial da produção de mésons vetoriais, que consegue reproduzir os dados experimentais dos mésons vetoriais $\rho, \omega, \phi$ e $J / \Psi$ e predizer o comportamento do méson $\Upsilon$.

- Para a interação dos mésons vetoriais com o meio nuclear, foram introduzidos os canais relacionados com as reações de espalhamento elásticas dos mésons com os nucleons e as colisões de espalhamento inelásticos. Os estados finais de $\omega N \rightarrow$ $\omega N, \rightarrow \pi N, \rightarrow \rho N, \rightarrow \rho \pi N, \rightarrow \pi \pi N$, foram calculados usando o modelo da aproximação troca de bóson desenvolvido por G.I. Lykasov, W. Cassing, A. Sibirtsev e M.V. Rzjanin. Este modelo está baseado no vértice de interação $\omega \rho \pi$, no qual a constante de acoplamento e o fator de forma são fixados pela reação $\pi N \rightarrow \omega N$ em comparação com os dados experimentais. A produção de mésons pela interação dos mésons $\pi$ com nucleons também é incluída usando o ajuste desenvolvido por A. Sibirtsev, W. Cassing e U. Mosel usando o modelo ressonante. Para o méson $J / \Psi$, o modelo CRISP inclui os processos de dissociação pela interação com nucleons usando o modelo desenvolvido por A. Sibirtsev, K. Tsushima e A. W. Thomas.

Estes canais junto com os canais inversos, definidos pelo princípio de balanço detalhado, cobrem quase a totalidade das interações dos mésons com os nucleons e assim, de acordo ao modelo CRISP, uma razoavelmente correta interação no estado final do méson.

A nova versão do código foi usada para o estudo de vários fenômenos produzidos nas fotorreações em altas energias e comparada com dados experimentais. A arquitetura do código CRISP, a linguagem em que é desenvolvido e a forma de inclusão de canais no CRISP permitem estudar vários fenômenos usando várias técnicas Monte Carlo que mostram seu potencial nos resultados. Algumas destas técnicas são métodos de redução de variância e a possibilidade de "ligar" e "desligar" canais de interação, modificando a FSI e assim aprofundar a análises dos processos. As conclusões de acordo aos resultados obtidos com esta nova versão do CRISP, são expostos a frente.

1. Foi estudado o efeito de sombreamento a partir da dependência da seção de choque nuclear dos mésons $\rho, \omega$, e $\phi$ em função do número de massa A para o valor de energia $E_{\gamma}=9 \mathrm{GeV}$. Neste estudo foi verificada corretamente a propagação do fóton no núcleo e analisado o efeito de sombreamento a energias longe do limiar de produção dos méson. Ajustando estes resultados foi obtido a seção de choque 
por nucleon $\sigma_{0}$ e o numero de massa efetivo $A_{e f f}=A^{\alpha}$. Este último reportou um valor $\sim A^{0.9}$ para todos os mésons estudados de acordo à literatura e foi usado para o cálculo da seção de choque por nucleon de produção em função da energia desde o limiar ate vários $G e V$. Neste estudo foi obtida uma boa concordância com os dados experimentais.

2. Foi estudada a produção sub-limiar do méson $J / \Psi$. Este fenômeno é determinado pela superposição de vários efeitos nucleares. Devido a isso foi analisado o mecanismo de bloqueio de Pauli que sugeriu o caráter não constante do expoente $\alpha$ do número de massa efetivo nas energias perto do limiar e o movimento fermiônico que introduz uma flutuação energética no sistema $\gamma N$ que permite a produção sublimiar. O comportamento deste expoente foi quantitativamente estudado usando técnicas de redução de variância para aumentar a taxa de produção do méson $J / \Psi$ sem modificar a seção de choque total de fotoprodução e com ele o efeito de sombreamento. O método usado neste estudo foi o mesmo, para várias energias, que o método explicado acima onde foi obtido o expoente do número de massa efetivo pelo ajuste do comportamento da seção de choque em função do número de massa A. Este método revelou um expoente que diminui com o aumento da energia na forma $\alpha\left(E_{\gamma}\right)=\alpha_{0}+e^{\beta\left(E_{\gamma}-E_{\gamma_{0}}\right)}$. Note-se a forma exponencial similar ao comportamento do bloqueio de Pauli em correspondência com um efeito de sombreamento não saturado. Este novo expoente foi usado para a obtenção da seção de choque de produção sub-limiar do méson $J / \Psi$ por nucleon que cai mais abruptamente do que a seção de choque normalizada a um expoente constante correspondente a um efeito de sombreamento saturado.

3. A caraterística dependência temporal do modelo de cascata CRISP foi usada para o estudo do decaimento do sistema $J / \Psi$-núcleo formado numa reação fotonuclear. Neste estudo foi analisado o processo de decaimento através de dois processos, pela emissão do méson fora do núcleo e pela dissociação deste pela interação com os nucleons. Cada um destes processos foi estudado com e sem FSI. O estudo revelou que o decaimento se produz fundamentalmente pela interação com os nucleons com uma taxa de produção $~ 98 \%$. Uma comparação entre os tempo de decaimento do processo de emissão do méson com e sem FSI mostrou que os mésons que conseguem sair do núcleo são aqueles que são produzidos perto da superfície nuclear.

4. O modelo CRISP inclui um mecanismo para simular o efeito quântico da distribuição de massa dos mésons produzidos no processo de fotoprodução. A distribuição de massa do méson foi estudada no ambiente nuclear. O estudo revelou que o bloqueio de Pauli tem um papel importante nesta grandeza. O mecanismo de 
Pauli demostrou ser mais intenso nas fotorreações onde a massa do méson é maior que se traduz num pequeno deslocamento do espectro para as massa menores. $\mathrm{O}$ motivo deste deslocamento é que os processos de fotoprodução de mésons mais pesados, pela conservação da energia, irão produzir um núcleon de menor energia correspondente a camadas mais baixas, e.i. ocupadas, da estruturas de camadas CRISP.

5. Todos os fenômenos anteriores se juntam na fotoprodução nuclear de mésons vetoriais e evidenciando sua alta complexidade. Este processo foi estudado neste trabalho pela uma grandeza que inclui todos estes efeitos nucleares substituindo o núcleo por "caixa preta": a taxa de transparência do mésons $\omega$. A transparência descreve a atenuação do fluxo de méson no meio nuclear. O estudo foi realizado com e sem a produção secundária do méson. Não foi obtido um resultado de acordo com os dados experimentais, de fato a taxa de transparência de nossos cálculos resultou maior do que o experimento. Esto indica que algum processo importante de absorção do méson ainda não está sendo considerado corretamente. Nós continuamos aprofundando este estudo na atualidade.

Uma das maiores motivações deste trabalho foi o estudo das colisões ultraperiféricas por sua atualidade e importância. Vários experimentos sobre o tema começaram a ser já estudados no LHC. Foi feita uma pesquisa bibliográfica sobre colisões ultraperiféricas na faixa de energia do LHC e foram detalhadas as características desta. Neste ponto pode-se concluir que é possível a simulação de um mecanismo de inicialização da cascata intranuclear substituindo o campo eletromagnético por uma distribuição de WeizsäckerWilliams de fótons reais que dependerá do valor da energia de centro de massa e do parâmetro de impacto da colisão. O mecanismo que inicia a cascata induzida por uma colisão ultraperiférica foi criado e o fluxo de fótons foi corretamente simulado.

Como próximo passo deste trabalho será estudada das colisões ultraperiféricas e se aprofundará o estudo da fotoprodução de mésons vetoriais. Outras simulações poderão ser feitas como complemento ao trabalho como a continuação da análise da interação no estado final do hyperon, assim como a física dos hypernúcleos, estudo que foi inicializado como trabalho prévio a este para a defesa de mestrado. Também poderão ser estudados os processos de fotoprodução sub-limiar para os mésons $\rho, \omega$ e $\phi$ usando o mesmo método que o usado para o estudo com o méson $J / \Psi$. 


\section{Referências Bibliográficas}

[1] V. Muccifora, N. Bianchi, A. Deppman, E. De Sanctis, M. Mirazita, E. Polli, P. Rossi, R. Burgwinkel, J. Hannappel, F. Klein, D. Menze, W. J. Schwille, and F. Wehnes. Photoabsorption on nuclei in the energy range $0.5^{\triangleleft} 2.6$ gev. Phys. Rev. C, 60:064616, Nov 1999. doi: 10.1103/PhysRevC.60.064616. URL http: //link.aps.org/doi/10.1103/PhysRevC.60.064616.

[2] M. Anghinolfi, V. Lucherini, P. Rossi, N. Bianchi, P. Corvisiero, A. Deppman, E. De Sanctis, A. Ebolese, A. Fantoni, G. Gervino, H. A. Gimm, P. Levi Sandri, L. Mazzaschi, V. Mokeev, V. Muccifora, E. Polli, A. R. Reolon, G. Ricco, M. Ripani, M. Sanzone, M. Taiuti, G. M. Urciuoli, and A. Zucchiatti. Behavior of the be and c total photonuclear cross section in the nucleon resonance region. Phys. Rev. C, 47:R922-R925, Mar 1993. doi: 10.1103/PhysRevC.47.R922. URL http://link.aps.org/doi/10.1103/PhysRevC.47.R922.

[3] M. Anghinolfi, N. Bianchi, P. Corvisiero, A. Deppman, E. De Sanctis, A. Ebolese, A. Fantoni, G. Gervino, P. Levi Sandri, V. Lucherini, L. Mazzaschi, V. Mokeev, V. Muccifora, E. Polli, A.R. Reolon, G. Ricco, M. Ripani, P. Rossi, M. Sanzone, M. Taiuti, and A. Zucchiatti. Total photonuclear cross section in the nucleon resonance region. Nuclear Physics A, 553(0):631-634, 1993. ISSN 03759474. doi: 10.1016/0375-9474(93)90670-S. URL http://www. sciencedirect. com/science/article/pii/037594749390670S.

[4] N. Bianchi, V. Muccifora, A. Deppman, E. De Sanctis, A. Fantoni, P. Levi Sandri, V. Lucherini, M. Mirazita, E. Polli, A.R. Reolon, P. Rossi, M. Anghinolfi, P. Corvisiero, G. Gervino, L. Mazzaschi, V. Mokeev, G. Ricco, M. Ripani, M. Sanzone, M. Taiuti, and A. Zucchiatti. Absolute measurement of the total photoabsorption cross section for carbon in the nucleon resonance region. Physics Letters B, 309(1-2):5-9, 1993. ISSN 0370-2693. doi: 10.1016/0370-2693(93)91494-8. URL http://www.sciencedirect.com/science/article/pii/0370269393914948.

[5] N. Bianchi, E. De Sanctis, M. Mirazita, and V. Muccifora. Shadowing in nuclear photoabsorption above the resonance region. Phys. Rev. C, 60:064617, Nov 1999. 
doi: 10.1103/PhysRevC.60.064617. URL http://link.aps.org/doi/10.1103/ PhysRevC.60.064617.

[6] M. Gonçalves, de Oliveira E.C., Medeiros E.L., de Pina S., and Duarte S.B. Hot hypernucleus formation in high-energy photonuclear reactions. Brazilian Journal of Physics, 34:919-923, 09 2004. ISSN 0103-9733. URL http://www.scielo.br/scielo.php?pid=S0103-97332004000500057script= sci $\{\%$ textunderscore $\}$ abstract.

[7] A.J. Baltz, G. Baur, D. d'Enterria, L. Frankfurt, F. Gelis, V. Guzey, K. Hencken, Yu. Kharlov, M. Klasen, S.R. Klein, V. Nikulin, J. Nystrand, I.A. Pshenichnov, S. Sadovsky, E. Scapparone, J. Seger, M. Strikman, M. Tverskoy, R. Vogt, S.N. White, U.A. Wiedemann, P. Yepes, and M. Zhalov. The physics of ultraperipheral collisions at the lhc. Physics Reports, 458(1-3):1-171, 2008. ISSN 0370-1573. doi: 10.1016/j.physrep.2007.12.001. URL http://www.sciencedirect.com/science/ article/pii/S0370157307004462.

[8] E. Abbas et al. Charmonium and $e^{+} e$ pair photoproduction at midrapidity in ultra-peripheral $\mathrm{Pb}-\mathrm{Pb}$ collisions at $\sqrt{s_{-} N N}=2.76 \mathrm{TeV}$. The European Physical Journal C, 73(11):1-18, 2013. ISSN 1434-6044. doi: 10.1140/epjc/s10052-013-2617-1. URL http://dx.doi.org/10.1140/epjc/ s10052-013-2617-1.

[9] I. A. Pshenichnov, I. N. Mishustin, J. P. Bondorf, A. S. Botvina, and A. S. Iljinov. Particle emission following coulomb excitation in ultrarelativistic heavy-ion collisions. Phys. Rev. C, 60:044901, Sep 1999. doi: 10.1103/PhysRevC.60.044901. URL http://link.aps.org/doi/10.1103/PhysRevC.60.044901.

[10] G. Sampaio dos Santos and M. V. T. Machado. Exclusive photoproduction of quarkonium in proton-nucleus collisions at energies available at the cern large hadron collider. Phys. Rev. C, 89:025201, Feb 2014. doi: 10.1103/PhysRevC.89. 025201. URL http://link.aps.org/doi/10.1103/PhysRevC.89.025201.

[11] M. H. Wood et al. Mesons in the medium: Experiments with clas at jefferson lab. International Journal of Modern Physics A, 26(3-4):734-736, FEB 10 2011. ISSN 0217-751X. doi: 10.1142/S0217751X11052682. URL http: //www. worldscientific.com/doi/abs/10.1142/S0217751X11052682.

[12] Paul Eugenio et al. Search for exotic mesons in photoproduction at jlab clas. In B Fleming, editor, 11TH CONFERENCE ON THE INTERSECTIONS OF PARTICLE AND NUCLEAR PHYSICS (CIPANP 2012), volume 1560 of AIP Conference Proceedings, pages 421-423, 2013. ISBN 978-0-7354-1188-3. doi: 10. $1063 / 1.4826808$. 
[13] M. H. Wood et al. Light vector mesons in the nuclear medium. Phys. Rev. C, 78:015201, Jul 2008. doi: 10.1103/PhysRevC.78.015201. URL http://link.aps . org/doi/10.1103/PhysRevC.78.015201.

[14] C. Djalali, M. Paolone, D. Weygand, M. H. Wood, and R. Nasseripour. The in-medium mass and widths of light vector mesons. volume 1343 of AIP Conference Proceedings, pages 574-576, 2011. ISBN 978-0-7354-0899-9. doi: http:// dx.doi.org/10.1063/1.3575098. URL http://scitation.aip.org/content/aip/ proceeding/aipcp/10.1063/1.3575098.

[15] Stanley J. Brodsky, L. Frankfurt, J. F. Gunion, A. H. Mueller, and M. Strikman. Diffractive leptoproduction of vector mesons in qcd. Phys. Rev. D, 50:3134-3144, Sep 1994. doi: 10.1103/PhysRevD.50.3134. URL http://link.aps.org/doi/10. 1103/PhysRevD. 50.3134.

[16] John C. Collins, Leonid Frankfurt, and Mark Strikman. Factorization for hard exclusive electroproduction of mesons in qcd. Phys. Rev. D, 56:2982-3006, Sep 1997. doi: 10.1103/PhysRevD.56.2982. URL http://link.aps.org/doi/10. 1103/PhysRevD.56.2982.

[17] A Deppman, S B Duarte, G Silva, O A P Tavares, S Anéfalos, J D T ArrudaNeto, and T E Rodrigues. The crisp package for intermediate- and high-energy photonuclear reactions. Journal of Physics G: Nuclear and Particle Physics, 30 (12):1991, 2004. URL http://stacks.iop.org/0954-3899/30/i=12/a=016.

[18] J. J. Sakurai. Vector-meson dominance and high-energy electron-proton inelastic scattering. Phys. Rev. Lett., 22:981-984, May 1969. doi: 10.1103/PhysRevLett. 22.981. URL http://link.aps.org/doi/10.1103/PhysRevLett.22.981.

[19] A. Baracca and A. Bramón. General analysis of the vector meson-dominance model predictions for meson decays. Il Nuovo Cimento A, 69(4):613-631, 1970. ISSN 0369-3546. doi: 10.1007/BF02819090. URL http://dx.doi.org/10.1007/ BF02819090.

[20] J.J Sakurai. Theory of strong interactions. Annals of Physics, 11(1):1-48, 1960. ISSN 0003-4916. doi: 10.1016/0003-4916(60)90126-3. URL http://www . sciencedirect.com/science/article/pii/0003491660901263.

[21] Norman M. Kroll, T. D. Lee, and Bruno Zumino. Neutral vector mesons and the hadronic electromagnetic current. Phys. Rev., 157:1376-1399, May 1967. doi: 10.1103/PhysRev.157.1376. URL http://link.aps.org/doi/10.1103/PhysRev . 157.1376 . 
[22] T. E. Rodrigues. O modelo de cascata intranuclear mcmc e aplicações para o mecanismo do quase-dêuteron e a fotoprodução de pions em energias intermediárias e altas, tese de doutorado, USP, 2005.

[23] H. W. Bertini. Intranuclear-cascade calculation of the secondary nucleon spectra from nucleon-nucleus interactions in the energy range 340 to $2900 \mathrm{MeV}$ and comparisons with experiment. Phys. Rev., 188:1711-1730, Dec 1969. doi: 10.1103/PhysRev.188.1711. URL http://link.aps .org/doi/10.1103/PhysRev . 188.1711.

[24] Y. Yariv and Z. Fraenkel. Intranuclear cascade calculation of high-energy heavyion interactions. Phys. Rev. C, 20:2227-2243, Dec 1979. doi: 10.1103/PhysRevC. 20.2227. URL http://link.aps.org/doi/10.1103/PhysRevC.20.2227.

[25] Y. Yariv and Z. Fraenkel. Intranuclear cascade calculation of high energy heavy ion collisions: Effect of interactions between cascade particles. Phys. Rev. C, 24 : 488-494, Aug 1981. doi: 10.1103/PhysRevC.24.488. URL http://link.aps.org/ doi/10.1103/PhysRevC.24.488.

[26] Barashenkov V. S. Statistical modelling of intranuclear cascades initiated by high energy particles. JINR, P2:81-364., 1981.

[27] V. S. Barashenkov and V. D. Toneev. Interaction of high energy particles and nuclei with atomic nuclei. Atomizdat, 1972.

[28] V.S. Barashenkov, A.S. Ilinov, N.M. Sobolevskii, and V.D. Toneev. Interaction of particles and nuclei of high and superhigh energies with nuclei. Usp.Fiz.Nauk, 109:91-136, 1973. doi: 10.3367/UFNr.0109.197301d.0091.

[29] V.S. Barashenkov, Van Ngoc Le, L.G. Levchuk, Zh. Zh. Musulmanbekov, A.N. Sosnin, et al. 'CASCADE' PROGRAM COMPLEX FOR MONTE CARLO SIMULATION OF NUCLEAR PROCESSES INITIATED BY HIGH-ENERGY PARTICLES AND NUCLEI IN GASEOUS AND CONDENSED MATTER. Predmetnoe Mat. Obespechenie, JINR-P2-85-173, 1985.

[30] I. A. Pshenichnov, I. N. Mishustin, J. P. Bondorf, A. S. Botvina, and A. S. Iljinov. Nuclear multifragmentation induced by electromagnetic fields of ultrarelativistic heavy ions. Phys. Rev. C, 57:1920-1926, Apr 1998. doi: 10.1103/PhysRevC.57. 1920. URL http://link.aps.org/doi/10.1103/PhysRevC.57.1920.

[31] I.A. Pshenichnov, B.L. Berman, W.J. Briscoe, C. Cetina, G. Feldman, P. Heimberg, A.S. Iljinov, and I.I. Strakovsky. Intranuclear-cascade model calculation of photofission probabilities for actinide nuclei. The European Physical Journal A 
- Hadrons and Nuclei, 24(1):69-84, 2005. ISSN 1434-6001. doi: 10.1140/epja/ i2004-10130-9. URL http://dx.doi.org/10.1140/epja/i2004-10130-9.

[32] P. Golubev, V. Avdeichikov, K.G. Fissum, B. Jakobsson, I.A. Pshenichnov, W.J. Briscoe, G.V. O’Rielly, J. Annand, K. Hansen, L. Isaksson, H. Jäderström, M. Karlsson, M. Lundin, B. Schröder, and L. Westerberg. Pion emission in 2h, 12c, 27al() reactions at threshold. Nuclear Physics A, 806(1-4):216-229, 2008. ISSN 0375-9474. doi: http://dx.doi.org/10.1016/j.nuclphysa.2008.02.308. URL http://www.sciencedirect.com/science/article/pii/S0375947408004065.

[33] I.A. Pshenichnov. Electromagnetic excitation and fragmentation of ultrarelativistic nuclei. Physics of Particles and Nuclei, 42(2):215-250, 2011. ISSN 10637796. doi: 10.1134/S1063779611020067. URL http://dx.doi.org/10.1134/ S1063779611020067.

[34] Malyshkin, Yury, Pshenichnov, Igor, Mishustin, Igor, and Greiner, Walter. Modeling spallation reactions in tungsten and uranium targets with the geant4 toolkit*. EPJ Web of Conferences, 21:10006, 2012. doi: 10.1051/epjconf/20122110006. URL http://dx.doi.org/10.1051/epjconf/20122110006.

[35] E.V. Karpechev, I.A. Pshenichnov, T.L. Karavicheva, A.B. Kurepin, M.B. Golubeva, F.F. Guber, A.I. Maevskaya, A.I. Reshetin, V.V. Tiflov, N.S. Topilskaya, P. Cortese, G. Dellacasa, R. Arnaldi, N. De Marco, A. Ferretti, M. Gallio, A. Musso, C. Oppedisano, A. Piccotti, E. Scomparin, E. Vercellin, C. Cicalò, G. Puddu, E. Siddi, P. Szymanski, and I. Efthymiopoulos. Emission of forward neutrons by $158 \mathrm{a}$ gev indium nuclei in collisions with al, cu, sn and pb. $\mathrm{Nu}$ clear Physics A, 921(0):60-84, 2014. ISSN 0375-9474. doi: http://dx.doi.org/10. 1016/j.nuclphysa.2013.11.003. URL http://www.sciencedirect.com/science/ article/pii/S0375947413007859.

[36] A.S. Iljinov, I.A. Pshenichnov, N. Bianchi, E. De Sanctis, V. Muccifora, M. Mirazita, and P. Rossi. Extension of the intranuclear cascade model for photonuclear reactions at energies up to 10 gev. Nuclear Physics A, 616(3-4):575-605, 1997. ISSN 0375-9474. doi: http://dx.doi.org/10.1016/S0375-9474(96)00478-2. URL http://www.sciencedirect.com/science/article/pii/S0375947496004782.

[37] K.K. Gudima and S.G. Mashnik. Extension of the LAQGSM03.01 Code to Describe Photo-Nuclear Reactions up to Tens of GeV. pages 525-534, 2006. URL http://arxiv.org/abs/nucl-th/0607007.

[38] S.G. Mashnik, M.I. Baznat, K.K. Gudima, A.J. Sierk, and R.E. Prael. Extension of the CEM2k and LAQGSM codes to describe photo-nuclear reactions. 2005. URL http://arxiv.org/abs/nucl-th/0503061. 
[39] Yu. E. Titarenko, V. F. Batyaev, A. Yu. Titarenko, M. A. Butko, K. V. Pavlov, S. N. Florya, R. S. Tikhonov, S. G. Mashnik, A. V. Ignatyuk, N. N. Titarenko, W. Gudowski, M. Těšínský, C.-M. L. Persson, H. Ait Abderrahim, H. Kumawat, and H. Duarte. Cross sections for nuclide production in a ${ }^{56} \mathrm{fe}$ target irradiated by $300,500,750,1000,1500$, and 2600 mev protons compared with data on a hydrogen target irradiated by 300, 500, 750, 1000, and $1500 \mathrm{mev} /$ nucleon ${ }^{56} \mathrm{fe}$ ions. Phys. Rev. C, 78:034615, Sep 2008. doi: 10.1103/PhysRevC.78.034615. URL http://link.aps.org/doi/10.1103/PhysRevC.78.034615.

[40] A. Boudard, J. Cugnon, S. Leray, and C. Volant. Intranuclear cascade model for a comprehensive description of spallation reaction data. Phys. Rev. C, 66:044615, Oct 2002. doi: 10.1103/PhysRevC.66.044615. URL http://link.aps.org/doi/ 10.1103/PhysRevC.66.044615.

[41] J. Cugnon, C. Volant, and S. Vuillier. Improved intranuclear cascade model for nucleon-nucleus interactions. Nuclear Physics A, 620(4):475-509, 1997. ISSN 0375-9474. doi: http://dx.doi.org/10.1016/S0375-9474(97)00186-3. URL http: //www.sciencedirect.com/science/article/pii/S0375947497001863.

[42] M. Gonçalves, S. de Pina, D.A. Lima, W. Milomen, E.L. Medeiros, and S.B. Duarte. Many-body cascade calculation for photonuclear reactions. Physics Letters B, 406(1-2):1-6, 1997. ISSN 0370-2693. doi: 10. 1016/S0370-2693(97)00662-X. URL http://www.sciencedirect.com/science/ article/pii/S037026939700662X.

[43] A. Boudard, J. Cugnon, J.-C. David, S. Leray, and D. Mancusi. New potentialities of the liège intranuclear cascade model for reactions induced by nucleons and light charged particles. Phys. Rev. C, 87:014606, Jan 2013. doi: 10.1103/PhysRevC.87. 014606. URL http://link.aps.org/doi/10.1103/PhysRevC.87.014606.

[44] A. Deppman, G. Silva, S. Anefalos, S. B. Duarte, F. García, F. H. Hisamoto, and O. A. P. Tavares. Photofission and total photoabsorption cross sections in the energy range of shadowing effects. Phys. Rev. C, 73:064607, Jun 2006. doi: 10.1103/PhysRevC.73.064607. URL http://link.aps.org/doi/10.1103/ PhysRevC.73.064607.

[45] S. A. Pereira, A. Deppman, G. Silva, J. R. Maiorino, A. dos Santos, S. B. Duarte, O. A. P. Tavares, and F. Garcia. Spallation product distributions and neutron multiplicities for accelerator-driven system using the CRISP code. Nucl. Sci. Eng., 159:102, 2008. URL http://www.ans.org/store/j_2763.

[46] S. A. Pereira, A. Deppman, G. Silva, J. R. Maiorino, A. dos Santos, S. B. Duarte, O. A. P. Tavares, and F. Garcia. Spallation product distributions and neutron 
multiplicities for accelerator-driven system using the CRISP code. EPJ Web of Conferences, 21:10001, 2012.

[47] J. Cugnon, T. Mizutani, and J. Vandermeulen. Equilibration in relativistic nuclear collisions. A Monte Carlo calculation. Nuclear Physics A, 352(3):505-534, 1981. doi: 10.1016/0375-9474(81)90427-9. URL http://www.sciencedirect.com/ science/article/B6TVB-4730XPF-51/2/0e779bd7439f7a20baf280846610a5a8.

[48] J. Cugnon and P. Henrotte. The low-energy limit of validity of the intranuclear cascade model. The European Physical Journal A - Hadrons and Nuclei, 16(3): 393-407, 2003. ISSN 1434-6001. doi: 10.1140/epja/i2002-10096-6. URL http: //dx.doi.org/10.1140/epja/i2002-10096-6.

[49] S Leray, A Boudard, J Gugnon, R Legrain, and C Volant. Comparison of spallation neutron and residual nuclide production data with different intra-nuclear cascade models. In Kling, $\mathrm{A}$ and Barao, $\mathrm{F}$ and Nakagawa, $\mathrm{M}$ and Tavora, L and Vaz, P, editor, ADVANCED MONTE CARLO FOR RADIATION PHYSICS, PARTICLE TRANSPORT SIMULATION AND APPLICATIONS, pages 1111-1116, 2001. ISBN 3-540-41795-8. International Conference on Advanced Monte Carlo for Radiation Physics, Particle Transport Simulation and Applications, LISBON, PORTUGAL, OCT 23-26, 2000.

[50] Th. Aoust, J. Cugnon, and J. Wagemans. Production of radiotoxic isotopes in LBE spallation targets: recent extensions of the INCL4 model and experimental validation. In Bersillon, $\mathrm{O}$ and Gunsing, $\mathrm{F}$ and Bauge, $\mathrm{E}$ and Jacqmin, $\mathrm{R}$ and Leray, S, editor, INTERNATIONAL CONFERENCE ON NUCLEAR DATA FOR SCIENCE AND TECHNOLOGY, VOL 2, PROCEEDINGS, pages 12011204. CEA; OECD Nucl Energy Agcy, 2008. ISBN 978-2-7598-0091-9. doi: \{10.1051/ndata:07768\}. International Conference on Nuclear Data for Science and Technology, Nice, FRANCE, APR 22-27, 2007.

[51] A Boudard et al. Spallation study with proton beams around $1 \mathrm{GeV}$ : neutron production. Nuc. Phisc. A, 663:1061C-1064C, JAN 31 2000. ISSN 0375-9474. doi: \{10.1016/S0375-9474(99)00770-8\}. 15th International Conference on Particles and Nuclei, UPPSALA, SWEDEN, JUN 10-16, 1999.

[52] S. de Pina, E.C. de Oliveira, E.L. Medeiros, S.B. Duarte, and M. Gonçalves calves calves calves. Photonuclear K+ production calculation near threshold. Phys.Lett., B434:1-6, 1998. doi: 10.1016/S0370-2693(98)00726-6. URL http://www . sciencedirect.com/science/article/pii/S0370269398007266.

[53] A. Deppman, O. A. P. Tavares, S. B. Duarte, E. C. de Oliveira, J. D. T. ArrudaNeto, S. R. de Pina, V. P. Likhachev, O. Rodriguez, J. Mesa, and M. Gonçalves. 
Photofissility of actinide nuclei at intermediate energies. Phys. Rev. Lett., 87: 182701, Oct 2001. doi: 10.1103/PhysRevLett.87.182701. URL http://link.aps . org/doi/10.1103/PhysRevLett.87.182701.

[54] A. Deppman, O.A.P. Tavares, S.B. Duarte, E.C. de Oliveira, J.D.T. ArrudaNeto, S.R. de Pina, V.P. Likhachev, O. Rodriguez, J. Mesa, and M. Gonçalves. The $\{\mathrm{MCEF}\}$ code for nuclear evaporation and fission calculations. Computer Physics Communications, 145(3):385-394, 2002. ISSN 0010-4655. doi: 10. 1016/S0010-4655(02)00278-3. URL http://www.sciencedirect.com/science/ article/pii/S0010465502002783.

[55] A. Deppman, O. A. P. Tavares, S. B. Duarte, J. D. T. Arruda-Neto, M. Gonçalves, V. P. Likhachev, and E. C. de Oliveira. Photofissility of heavy nuclei at intermediate energies. Phys. Rev. C, 66:067601, Dec 2002. doi: 10.1103/PhysRevC.66. 067601. URL http://link.aps.org/doi/10.1103/PhysRevC.66.067601.

[56] Tulio E. Rodrigues and Joao Dias de Toledo Arruda-Neto. Vector Mesons in Cold Nuclear Matter. In Li, BA and Natowitz, JB, editor, 11TH INTERNATIONAL CONFERENCE ON NUCLEUS-NUCLEUS COLLISIONS (NN2012), volume 420 of Journal of Physics Conference Series. Texas A\&M Univ-Commerce, Dept Phys and Astron, 2013. doi: \{10.1088/1742-6596/420/1/012014\}. 11th International Conference on Nucleus-Nucleus Collisions (NN), Texas A\&M Univ, Cyclotron Inst, San Antonio, TX, MAY 27-JUN 01, 2012.

[57] B. M. Santos, M. Gonçalves calves calves calves, L. P. G. de Assis, and S. B. Duarte. Spallation process with simultaneous multi-particle emission in nuclear evaporation. In Melquiades, FL and Genezini, FA and Medina, NH and DosAnjos, RM and Avancini, SD, editor, XXXV BRAZILIAN WORKSHOP ON NUCLEAR PHYSICS, volume 1529 of AIP Conference Proceedings, pages 214-216, 2013. ISBN 978-0-7354-1154-8. doi: \{10.1063/1.4804120\}. 35th Brazilian Workshop on Nuclear Physics, Sao Sebastiao, BRAZIL, SEP 02-06, 2012.

[58] T. E. Rodrigues and J. D. T. Arruda-Neto. Forward-angle incoherent photoproduction of pseudoscalar mesons off nuclei. Phys. Rev. C, 86:037601, Sep 2012. doi: 10.1103/PhysRevC.86.037601. URL http://link.aps.org/doi/10.1103/ PhysRevC. 86.037601.

[59] S. B. Duarte. Reações nucleares relativísticas e o método de cascata intranuclear, tese de doutorado, CBPF, 1983.

[60] K.K. Gudima, S.G. Mashnik, and V.D. Toneev. Cascade-exciton model of nuclear reactions. Nucl.Phys., A401:329-361, 1983. doi: http://dx.doi.org/10. 
1016/0375-9474(83)90532-8. URL http://www.sciencedirect.com/science/ article/pii/0375947483905328.

[61] E. L. Medeiros, S. Duarte, and T. Kodama. The Effect of Nuclear Binding Energy on Pion Yields in Relativistic Nuclear Collisions. Phys.Lett., B203:205, 1988. doi: 10.1016/0370-2693(88)90539-4. URL http://www. sciencedirect. com/science/article/pii/S0370269398007266.

[62] Kim Sneppen and Carl Gaarde. Cascade simulation of a $\delta$ isobar propagating in a nucleus. Phys. Rev. C, 50:338-349, Jul 1994. doi: 10.1103/PhysRevC.50.338. URL http://link.aps.org/doi/10.1103/PhysRevC.50.338.

[63] M. Gonçalves. Efeitos de colisões a muitos corpos na produção de partículas em reações nucleares relativísticas, tese de doutorado, cbpf, 1996.

[64] T. Kodama, S. B. Duarte, K. C. Chung, and R. A. M. S. Nazareth. Cluster approach to intranuclear cascade for relativistic heavy-ion collisions. Phys. Rev. Lett., 49:536-539, Aug 1982. doi: 10.1103/PhysRevLett.49.536. URL http:// link.aps.org/doi/10.1103/PhysRevLett.49.536.

[65] Brian D. Serot and John Dirk Walecka. The Relativistic Nuclear Many Body Problem. Adv.Nucl.Phys., 16:1-327, 1986.

[66] E. Andrade-II, J C M Menezes, S B Duarte, F Garcia, P C R Rossi, O A P Tavares, and A. Deppman. Fragment mass distributions in the fission of heavy nuclei by intermediate- and high-energy probes. Journal of Physics G: Nuclear and Particle Physics, 38(8):085104, 2011. URL http://stacks.iop.org/0954-3899/38/i=8/ $\mathrm{a}=085104$.

[67] A. Deppman, E. Andrade-II, V. Guimarães, G. S. Karapetyan, O. A. P. Tavares, A. R. Balabekyan, N. A. Demekhina, J. Adam, F. Garcia, and K. Katovsky. Superasymmetric fission of heavy nuclei induced by intermediate-energy protons. Phys. Rev. C, 88:064609, Dec 2013. doi: 10.1103/PhysRevC.88.064609. URL http://link.aps.org/doi/10.1103/PhysRevC.88.064609.

[68] E. Andrade-II, E. Freitas, O. A. P. Tavares, et al. Xxxi workshop on nuclear physics in brazil (aip, new york, 2009). AIP Conf. Proc., 1139:64, 2009.

[69] V.P. Likhachev, J. Mesa, J.D.T. Arruda-Neto, B.V. Carlson, W.R. Carvalho Jr., L.C. Chamon, A. Deppman, H. Dias, and M.S. Hussein. Quasifree $238 \mathrm{u}(\mathrm{e}, \mathrm{ef})$ cross section in macroscopic-microscopic approach. Nuclear Physics A, 713(1-2):24-44, 2003. ISSN 0375-9474. doi: http://dx.doi.org/10. 1016/S0375-9474(02)01302-7. URL http://www.sciencedirect.com/science/ article/pii/S0375947402013027. 
[70] V. P. Likhachev, J. D. T. Arruda-Neto, W. R. Carvalho, A. Deppman, I. G. Evseev, F. Garcia, M. S. Hussein, L. F. R. Macedo, A. Margaryan, J. Mesa, V. O. Nesterenko, O. Rodriguez, S. A. Pashchuk, H. R. Schelin, and M. S. Vaudeluci. Inclusive quasifree electrofission cross section for ${ }^{238} \mathrm{u}$. Phys. Rev. C, 68:014615, Jul 2003. doi: 10.1103/PhysRevC.68.014615. URL http://link.aps.org/doi/ 10.1103/PhysRevC.68.014615.

[71] S. de Pina, E.C. de Oliveira, E.L. Medeiros, S.B. Duarte, and M. Gonçalves. Photonuclear $\mathrm{k}+$ production calculation near threshold. Physics Letters $B$, 434(1-2):1-6, 1998. ISSN 0370-2693. doi: http://dx.doi.org/10. 1016/S0370-2693(98)00726-6. URL http://www.sciencedirect.com/science/ article/pii/S0370269398007266.

[72] I Gonzalez, C Barbero, A Deppman, S B Duarte, F Krmpotić, and O Rodriguez. Many-body cascade calculation of final state interactions in ${ }^{12}{ } \Lambda \mathrm{C}$ nonmesonic weak decay. Journal of Physics G: Nuclear and Particle Physics, 38(11):115105, 2011. URL http://stacks .iop.org/0954-3899/38/i=11/a=115105.

[73] I Gonzalez, A Deppman, S Duarte, F Krmpotić, M S Hussein, and C Barbero. Nonmesonic hyperon weak decay spectra in 12 c. Journal of Physics: Conference Series, 312(2):022017, 2011. URL http://stacks.iop.org/1742-6596/312/i= $2 / a=022017$.

[74] Franjo Krmpotić, Airton Deppman, and Claudio De Conti. Dynamics of hypernuclear nonmesonic weak decay. AIP Conference Proceedings, 1491(1), 2012.

[75] A. Deppman, O.A.P. Tavares, S.B. Duarte, J.D.T. Arruda-Neto, M. Gonçalves, V.P. Likhachev, J. Mesa, E.C. de Oliveira, S.R. de Pina, and O. Rodriguez. A monte carlo method for nuclear evaporation and fission at intermediate energies. Nuclear Instruments and Methods in Physics Research Section B: Beam Interactions with Materials and Atoms, 211(1):15-21, 2003. ISSN 0168-583X. doi: http:// dx.doi.org/10.1016/S0168-583X(03)01265-5. URL http://www.sciencedirect . com/science/article/pii/S0168583X03012655.

[76] Sakshi Gautam, Aman D. Sood, Rajeev K. Puri, and J. Aichelin. Sensitivity of the transverse flow to the symmetry energy. Phys. Rev. C, 83:034606, Mar 2011. doi: 10.1103/PhysRevC.83.034606. URL http://link.aps.org/doi/10.1103/ PhysRevC. 83.034606.

[77] Yu-Ming Zheng, C. M. Ko, Bao-An Li, and Bin Zhang. Elliptic flow in heavyion collisions near the balance energy. Phys. Rev. Lett., 83:2534-2536, Sep 1999. doi: 10.1103/PhysRevLett.83.2534. URL http://link.aps.org/doi/10.1103/ PhysRevLett. 83.2534. 
[78] Sanjeev Kumar, Suneel Kumar, and Rajeev K. Puri. Elliptical flow and isospin effects in heavy-ion collisions at intermediate energies. Phys. Rev. C, 81:014611, Jan 2010. doi: 10.1103/PhysRevC.81.014611. URL http://link.aps.org/doi/ 10.1103/PhysRevC.81.014611.

[79] Sakshi Gautam and Aman D. Sood. Isospin effects on the mass dependence of the balance energy. Phys. Rev. C, 82:014604, Jul 2010. doi: 10.1103/PhysRevC.82. 014604. URL http://link.aps.org/doi/10.1103/PhysRevC.82.014604.

[80] D. Krofcheck, W. Bauer, G. M. Crawley, C. Djalali, S. Howden, C. A. Ogilvie, A. Vander Molen, G. D. Westfall, W. K. Wilson, R. S. Tickle, and C. Gale. Disappearance of flow in heavy-ion collisions. Phys. Rev. Lett., 63:2028-2031, Nov 1989. doi: 10.1103/PhysRevLett.63.2028. URL http://link.aps.org/doi/10. 1103/PhysRevLett.63.2028.

[81] Bao-An Li, Zhongzhou Ren, C. M. Ko, and Sherry J. Yennello. Isospin dependence of collective flow in heavy-ion collisions at intermediate energies. Phys. Rev. Lett., 76:4492-4495, Jun 1996. doi: 10.1103/PhysRevLett.76.4492. URL http://link. aps.org/doi/10.1103/PhysRevLett.76.4492.

[82] C. A. Ogilvie, W. Bauer, D. A. Cebra, J. Clayton, S. Howden, J. Karn, A. Nadasen, A. Vander Molen, G. D. Westfall, W. K. Wilson, and J. S. Winfield. Disappearance of flow and its relevance to nuclear matter physics. Phys. Rev. C, 42:R10-R14, Jul 1990. doi: 10.1103/PhysRevC.42.R10. URL http://link.aps.org/doi/10 . 1103/PhysRevC.42.R10.

[83] J. Eukasik et al. Directed and elliptic flow in ${ }^{197} \mathrm{Au}+{ }^{197} \mathrm{Au}$ at intermediate energies. Physics Letters B, 608(3-4):223-230, 2005. ISSN 0370-2693. doi: http: //dx.doi.org/10.1016/j.physletb.2004.12.076. URL http://www. sciencedirect. com/science/article/pii/S0370269304017708.

[84] Rajni, Suneel Kumar, and Rajeev K. Puri. Correlation between balance energy and transition energy for symmetric colliding nuclei. Phys. Rev. C, 84:037606, Sep 2011. doi: 10.1103/PhysRevC.84.037606. URL http://link.aps.org/doi/10. 1103/PhysRevC.84.037606.

[85] Z. Y. Sun et al. Isospin diffusion and equilibration for $\mathrm{Sn}+\mathrm{Sn}$ collisions at $\mathrm{E} / \mathrm{A}=35$ MeV. Phys. Rev. C, 82:051603, Nov 2010. doi: 10.1103/PhysRevC.82.051603. URL http://link.aps.org/doi/10.1103/PhysRevC.82.051603.

[86] F. Rami et al. Isospin tracing: A probe of nonequilibrium in central heavy-ion collisions. Phys. Rev. Lett., 84:1120-1123, Feb 2000. doi: 10.1103/PhysRevLett. 84.1120. URL http://link.aps.org/doi/10.1103/PhysRevLett.84.1120. 
[87] Rajeev K. Puri, Christoph Hartnack, and Jörg Aichelin. Early fragment formation in heavy-ion collisions. Phys. Rev. C, 54:R28-R31, Jul 1996. doi: 10. 1103/PhysRevC.54.R28. URL http://link.aps.org/doi/10.1103/PhysRevC. 54.R28.

[88] Sanjeev Kumar, Suneel Kumar, and Rajeev K. Puri. Medium mass fragment production due to momentum dependent interactions. Phys. Rev. C, 78:064602, Dec 2008. doi: 10.1103/PhysRevC.78.064602. URL http://link.aps.org/doi/ 10.1103/PhysRevC.78.064602.

[89] S.W. Huang, Amand Faessler, G.Q. Li, Rajeev K. Puri, E. Lehmann, Dao T. Khoa, and M.A. Matin. Subthreshold K+ production in $1 \mathrm{GeV} / \mathrm{U}{ }^{197} \mathrm{Au}+{ }^{197} \mathrm{Au}$ collisions. Physics Letters B, 298(1):41, 1993. ISSN 0370-2693. doi: http://dx. doi.org/10.1016/0370-2693(93)91703-P. URL http://www.sciencedirect.com/ science/article/pii/037026939391703P.

[90] W. Bauer. Nuclear stopping at intermediate beam energies. Phys. Rev. Lett., 61: 2534-2537, Nov 1988. doi: 10.1103/PhysRevLett.61.2534. URL http://link. aps.org/doi/10.1103/PhysRevLett.61.2534.

[91] C. A. García Canal, E. M. Santangelo, and H. Vucetich. Nucleon effective mass and the a dependence of structure functions. Phys. Rev. Lett., 53:1430-1432, Oct 1984. doi: 10.1103/PhysRevLett.53.1430. URL http://link.aps.org/doi/10. 1103/PhysRevLett.53.1430.

[92] P. R. Rossi. Reações nucleares e alta energia ("spallation") e sua aplicação em calculo de sistemas nucleares acionados por fonte, tese de doutorado, IPEN, 2011.

[93] K. Kikuchi and M. Kawai. Nuclear Matter and Nuclear Reactions. North-Holland research monograph. North-Holland Pub. Co., 1968. URL http://books .google . com. br/books? id=WVQzAAAAMAAJ.

[94] J. Cugnon. Proton-nucleus interaction at high energy. Nuclear Physics A, 462(4):751-780, 1987. ISSN 0375-9474. doi: http://dx.doi.org/10. 1016/0375-9474(87)90575-6. URL http://www.sciencedirect.com/science/ article/pii/0375947487905756.

[95] J. Benlliure, P. Armbruster, M. Bernas, A. Boudard, T. Enqvist, et al. Signatures of fission dynamics in highly excited nuclei produced in ${ }^{197} \mathrm{Au}(800 \mathrm{~A}-\mathrm{MeV})$ on proton collisions. Nucl.Phys., A700:469-491, 2002. doi: http://dx.doi.org/10. 1016/S0375-9474(01)01331-8. URL http://www.sciencedirect.com/science/ article/pii/S0375947401013318. 
[96] W. Wlazło, T. Enqvist, P. Armbruster, J. Benlliure, M. Bernas, A. Boudard, S. Czájkowski, R. Legrain, S. Leray, B. Mustapha, M. Pravikoff, F. Rejmund, K.H. Schmidt, C. Stéphan, J. Taieb, L. Tassan-Got, and C. Volant. Cross sections of spallation residues produced in 1a gev p208b on proton reactions. Phys. Rev. Lett., 84:5736-5739, Jun 2000. doi: 10.1103/PhysRevLett.84.5736. URL http: //link.aps.org/doi/10.1103/PhysRevLett.84.5736.

[97] C. Toccoli. Ph.d. thesis university of orsay, 2000.

[98] Graham Shaw. The hadronic component of the photon and nucleon structure functions at small x. Physics Letters B, 318(1):221-226, $1993 . \quad$ ISSN 03702693. doi: 10.1016/0370-2693(93)91810-A. URL http://www.sciencedirect. com/science/article/pii/037026939391810A.

[99] Michele Arneodo. Nuclear effects in structure functions. Physics Reports, 240 (5-6):301-393, 1994. ISSN 0370-1573. doi: 10.1016/0370-1573(94)90048-5. URL http://www.sciencedirect.com/science/article/pii/0370157394900485.

[100] P Moseley and G Shaw. Hadron dominance revisited. Journal of Physics G: Nuclear and Particle Physics, 21(8):1043, 1995. URL http://stacks.iop.org/ 0954-3899/21/i=8/a=003.

[101] A. Deppman. Estado dos mecanismos de fotoabsorção do ${ }^{238} \mathrm{U}$ na região das ressonâncias nucleônicas, tese de doutorado, USP, 1993.

[102] W.D. Myers and W.J. Swiatecki. Anomalies in nuclear masses. Ark.Fys., 36: 343-352, 1967.

[103] V. Weisskopf. Statistics and nuclear reactions. Phys. Rev., 52:295-303, Aug 1937. doi: 10.1103/PhysRev.52.295. URL http://link.aps.org/doi/10.1103/ PhysRev.52.295.

[104] I. Dostrovsky, P. Rabinowitz, and R. Bivins. Monte carlo calculations of highenergy nuclear interactions. i. systematics of nuclear evaporation. Phys. Rev., 111: 1659-1676, Sep 1958. doi: 10.1103/PhysRev.111.1659. URL http://link.aps . org/doi/10.1103/PhysRev.111.1659.

[105] J R Nix. Calculation of fission barriers for heavy and superheavy nuclei. Annual Review of Nuclear Science, 22(1):65-120, 1972. doi: 10.1146/annurev. ns.22.120172.000433. URL http://www . annualreviews.org/doi/abs/10.1146/ annurev.ns.22.120172.000433.

[106] Vicenzon Barone and Enrico Predazzi. High energy particle diffraction. Springer, 2002. 
[107] T. Regge. Introduction to complex orbital momenta. Nuovo Cim., 14:951, 1959. doi: 10.1007/BF02728177. URL http://dx.doi.org/10.1007/BF02728177.

[108] A. Donnachie and P.V. Landshoff. Exclusive rho Production in Deep Inelastic Scattering. Phys.Lett., B185:403, 1987. doi: 10.1016/0370-2693(87)91024-0. URL http://www.sciencedirect.com/science/article/pii/0370269387910240.

[109] John C. Collins, Davison E. Soper, and George F. Sterman. Factorization of Hard Processes in QCD. Adv.Ser.Direct.High Energy Phys., 5:1-91, 1988. URL http://arxiv.org/abs/hep-ph/0409313.

[110] Goldberger M. L. and Watson K. M. Collision Theory. New York: Wiley, 1964.

[111] Marcel Froissart. Asymptotic behavior and subtractions in the mandelstam representation. Phys. Rev., 123:1053-1057, Aug 1961. doi: 10.1103/PhysRev.123.1053. URL http://link.aps.org/doi/10.1103/PhysRev.123.1053.

[112] A. Martin. Unitarity and high-energy behavior of scattering amplitudes. Phys. Rev., 129:1432-1436, Feb 1963. doi: 10.1103/PhysRev.129.1432. URL http: //link.aps.org/doi/10.1103/PhysRev.129.1432.

[113] A. A. Abrikosov, A. D. Galanin, L. P. Gorkov, L. D. Landau, I. Ya. Pomeranchuk, and K. A. Ter-Martirosyan. Possibility of formulation of a theory of strongly interacting fermions. Phys. Rev., 111:321-328, Jul 1958. doi: 10.1103/PhysRev. 111.321. URL http://link.aps.org/doi/10.1103/PhysRev.111.321.

[114] A. Donnachie and P.V. Landshoff. Total cross-sections. Phys.Lett., B296:227232, 1992. doi: http://dx.doi.org/10.1016/0370-2693(92)90832-O. URL http: //www.sciencedirect.com/science/article/pii/0370269392908320.

[115] G. Giacomelli, P. Lugaresi-Serra, G. Mandrioli, A. Minguzzi-Ranzi, F. Griffiths, I.S. Hughes, A.A. Hirata, R. Jennings, B.C. Wilson, G. Ciapetti, G. Mastrantonio, D. Zanello, G. Alberi, E. Castelli, P. Poropat, and M. Sessa. Coherent K+d interactions between 0.64 and $1.51 \mathrm{GeV} /$ c. Nuclear Physics B, 68(2):285-300, 1974. ISSN 0550-3213. doi: http://dx.doi.org/10.1016/0550-3213(74)90312-5. URL http://www.sciencedirect.com/science/article/pii/0550321374903125.

[116] Geoffrey F. Chew and S. C. Frautschi. Principle of equivalence for all strongly interacting particles within the s-matrix framework. Phys. Rev. Lett., 7:394-397, Nov 1961. doi: 10.1103/PhysRevLett.7.394. URL http://link.aps.org/doi/ 10.1103/PhysRevLett.7.394.

[117] V.N. Gribov. Possible Asymptotic Behavior of Elastic Scattering. JETP Lett., 41: 667-669, 1961. 
[118] S. Abatzis, F. Antinori, et al. Observation of a narrow scalar meson at 1450$\mathrm{MeV}$ in the reaction pp $\rightarrow p_{-} f\left(\pi^{+} \pi^{-} \pi^{+} \pi^{-}\right) \mathrm{p}_{-} s$ at $450-\mathrm{GeV} / \mathrm{c}$ using the CERN Omega Spectrometer. Physics Letters B, 324(3-4):509-514, 1994. ISSN 03702693. doi: http://dx.doi.org/10.1016/0370-2693(94)90231-3. URL http://www . sciencedirect.com/science/article/pii/0370269394902313.

[119] D. Amati, S. Fubini, and A. Stanghellini. Asymptotic properties of scattering and multiple production. Physics Letters, 1(1):29-32, 1962. ISSN 0031-9163. doi: http: //dx.doi.org/10.1016/0031-9163(62)90269-X. URL http://www. sciencedirect. com/science/article/pii/003191636290269X.

[120] S. Mandelstam. Cuts in the Angular Momentum Plane. 2. Nuovo Cim., 30: 1148-1162, 1963. doi: 10.1007/BF02828822. URL http://dx.doi.org/10.1007/ BF02828822.

[121] V.N. Gribov, I. Ya. Pomeranchuk, and K.A. Ter-Martirosian. Formation of mesic atoms in the decay of heavy hypernuclei. Sov.J.Nucl.Phys., 2:258-260, 1965.

[122] R.J.M. Covolan, J. Montanha, and K. Goulianos. A new determination of the soft pomeron intercept. Physics Letters B, 389(1):176-180, 1996. ISSN 03702693. doi: http://dx.doi.org/10.1016/S0370-2693(96)01362-7. URL http://www . sciencedirect.com/science/article/pii/S0370269396013627.

[123] Jean-René Cudell, Kyungsik Kang, and Sung Ku Kim. Bounds on the soft pomeron intercept. Physics Letters B, 395(3-4):311-317, 1997. ISSN 0370-2693. doi: http:// dx.doi.org/10.1016/S0370-2693(97)00046-4. URL http://www.sciencedirect . com/science/article/pii/S0370269397000464.

[124] E. A. Kuraev, L. N. Lipatov, and Victor S. Fadin. Multi - Reggeon Processes in the Yang-Mills Theory. Sov.Phys.JETP, 44:443-450, 1976.

[125] I.I. Balitsky and L.N. Lipatov. The Pomeranchuk Singularity in Quantum Chromodynamics. Sov.J.Nucl.Phys., 28:822-829, 1978.

[126] L.N. Lipatov. The Bare Pomeron in Quantum Chromodynamics. Sov.Phys.JETP, 63:904-912, 1986.

[127] L. Lukaszuk and B. Nicolescu. A Possible interpretation of p p rising total crosssections. Lett.Nuovo Cim., 8:405-413, 1973. doi: 10.1007/BF02824484. URL http://dx.doi.org/10.1007/BF02824484.

[128] L Frankfurt, M Strikman, and C Weiss. Small-x physics: From HERA to LHC and beyond. ANNUAL REVIEW OF NUCLEAR AND PARTICLE SCIENCE, 55:403-465, 2005. ISSN 0163-8998. doi: \{10.1146/annurev.nucl.53.041002. 
110615\}. URL http://www.annualreviews.org/doi/abs/10.1146/annurev . nucl.53.041002.110615.

[129] B. Blättel, G. Baym, L. L. Frankfurt, and M. Strikman. How transparent are hadrons to pions? Phys. Rev. Lett., 70:896-899, Feb 1993. doi: 10.1103/ PhysRevLett.70.896. URL http://link.aps.org/doi/10.1103/PhysRevLett. 70.896 .

[130] L. Frankfurt, A. Radyushkin, and M. Strikman. Interaction of a small-size wave packet with a hadron target. Phys. Rev. D, 55:98-104, Jan 1997. doi: 10.1103/ PhysRevD.55.98. URL http://link.aps.org/doi/10.1103/PhysRevD.55.98.

[131] M. Arneodo, A. Bialas, M. W. Krasny, T. Sloan, and M. Strikman. Nuclear beams in HERA. 1996. URL http://arxiv.org/abs/hep-ph/9610423.

[132] C.A. Bertulani and G. Baur. Electromagnetic processes in relativistic heavy ion collisions. Nuclear Physics A, 458(4):725-744, 1986. ISSN 0375-9474. doi: 10. 1016/0375-9474(86)90197-1. URL http://www.sciencedirect.com/science/ article/pii/0375947486901971.

[133] Carlos A. Bertulani and Gerhard Baur. Electromagnetic processes in relativistic heavy ion collisions. Physics Reports, 163(5-6):299-408, 1988. ISSN 03701573. doi: http://dx.doi.org/10.1016/0370-1573(88)90142-1. URL http://www . sciencedirect.com/science/article/pii/0370157388901421.

[134] C.A. Bertulani and G. Baur. Relativistic coulomb collisions and the virtual radiation spectrum. Nuclear Physics A, 442(3):739-752, 1985. ISSN 0375-9474. doi: 10.1016/S0375-9474(85)80039-7. URL http://www.sciencedirect.com/ science/article/pii/S0375947485800397.

[135] Aage Winther and Kurt Alder. Relativistic coulomb excitation. Nuclear Physics A, 319(3):518-532, 1979. ISSN 0375-9474. doi: 10.1016/0375-9474(79)90528-1. URL http://www.sciencedirect.com/science/article/pii/0375947479905281.

[136] E. Fermi. On the Theory of the impact between atoms and electrically charged particles. Z.Phys., 29:315-327, 1924.

[137] C.F.v. Weizsäcker. Radiation emitted in collisions of very fast electrons. Z.Phys., 88:612-625, 1934. doi: 10.1007/BF01333110. URL http://dx.doi.org/10.1007/ BF01333110.

[138] E. J. Williams. Nature of the high energy particles of penetrating radiation and status of ionization and radiation formulae. Phys. Rev., 45:729-730, May 
1934. doi: 10.1103/PhysRev.45.729. URL http://link.aps.org/doi/10.1103/ PhysRev. 45.729.

[139] J.D. Jackson. Classical Electrodynamics. John Wiley \& Sons, New York, 2nd ed. edition, 1975.

[140] P.D.B. Collins and E.J. Squires. Regge poles in particle physics. Springer tracts in modern physics. Springer, 1968. URL http://books.google.com.br/books? id=OGFFAAAAYAAJ.

[141] J.A. Crittenden. Exclusive production of neutral vector mesons at the electron proton collider HERA. 1997.

[142] A. Donnachie and P.V. Landshoff. Exclusive vector meson production at HERA. Phys.Lett., B348:213-218, 1995. doi: 10.1016/0370-2693(95)00115-2. URL http: //www.sciencedirect.com/science/article/pii/0370269395001152.

[143] L.P.A. Haakman, A. Kaidalov, and J.H. Koch. Production of vector mesons by real and virtual photons at high-energies. Phys.Lett., B365:411-417, 1996. doi: 10.1016/0370-2693(95)01267-2. URL http://www.sciencedirect.com/ science/article/pii/0370269395012672.

[144] V.A. Petrov and A.V. Prokudin. Regge eikonal approach and its off-shell extension versus experimental data. pages 95-108, 1999.

[145] Laszlo L. Jenkovszky, E.S. Martynov, and F. Paccanoni. Regge pole model for vector meson photoproduction at HERA. 1996.

[146] R. Fiore, Laszlo L. Jenkovszky, and F. Paccanoni. Photoproduction of heavy vector mesons at HERA: A Test field for diffraction. Eur.Phys.J., C10:461-467, 1999. doi: 10.1007/s100520050768. URL http://dx.doi.org/10.1007/s100529900046.

[147] Isabelle Royen and Jean-René Cudell. Fermi notion and quark off-shellness in elastic vector-meson production. Nuclear Physics B, 545(1-3):505-525, 1999. ISSN 0550-3213. doi: http://dx.doi.org/10.1016/S0550-3213(98)00858-X. URL http: //www.sciencedirect.com/science/article/pii/S055032139800858X.

[148] A. Donnachie, J. Gravelis, and G. Shaw. Unified model of exclusive $\rho 0, \varphi$, and J/ $\psi$ electroproduction. Phys. Rev. D, 63:114013, May 2001. doi: 10.1103/PhysRevD. 63.114013. URL http://link.aps.org/doi/10.1103/PhysRevD.63.114013.

[149] J. Nemchik, Nikolai N. Nikolaev, E. Predazzi, and B.G. Zakharov. Color dipole phenomenology of diffractive electroproduction of light vector mesons at HERA. Z.Phys., C75:71-87, 1997. doi: 10.1007/s002880050448. URL http://arxiv.org/ abs/hep-ph/9605231. 
[150] J.R Cudell and I Royen. Elastic vector-meson production at $\{$ HERA . Physics Letters B, 397(3-4):317-324, 1997. ISSN 0370-2693. doi: http://dx.doi.org/10. 1016/S0370-2693(97)00193-7. URL http://www.sciencedirect.com/science/ article/pii/S0370269397001937.

[151] E. Martynov, E. Predazzi, and A. Prokudin. A Universal Regge pole model for all vector meson exclusive photoproduction by real and virtual photons. Eur.Phys.J., C26:271-284, 2002. doi: 10.1140/epjc/s2002-01058-5. URL http://dx.doi.org/ 10.1140/epjc/s2002-01058-5.

[152] E. Martynov, E. Predazzi, and A. Prokudin. Photoproduction of vector mesons in the soft dipole pomeron model. Phys. Rev. D, 67:074023, Apr 2003. doi: 10.1103/ PhysRevD.67.074023. URL http://link.aps.org/doi/10.1103/PhysRevD.67. 074023.

[153] J.R. Cudell, A. Donnachie, and P.V. Landshoff. Perturbative evolution and regge behaviour. Physics Letters B, 448(3-4):281-289, 1999. ISSN 03702693. doi: http://dx.doi.org/10.1016/S0370-2693(99)00063-5. URL http://www . sciencedirect.com/science/article/pii/S0370269399000635.

[154] P. Desgrolard and E. Martynov. Regge models of the proton structure function with and without hard pomeron: A Comparative analysis. Eur.Phys.J., C22: 479-492, 2001. doi: 10.1007/s100520100811.

[155] G. Zweig. An SU3 model for strong interaction symmetry and its breaking. CERN.Report, 8419/Th:412, 1964.

[156] S. Okubo. -meson and unitary symmetry model $\phi . \quad$ Physics Letters, 5(2):165-168, 1963. ISSN 0031-9163. doi: http://dx.doi.org/10. 1016/S0375-9601(63)92548-9. URL http://www.sciencedirect.com/science/ article/pii/S0375960163925489.

[157] Susumu Okubo. Consequences of quark-line (okubo-zweig-iizuka) rule. Phys. Rev. D, 16:2336-2352, Oct 1977. doi: 10.1103/PhysRevD.16.2336. URL http://link. aps.org/doi/10.1103/PhysRevD.16.2336.

[158] Jugoro Iizuka, Kunihiko Okada, and Okiyasu Shito. Systematics and phenomenology of boson mass levels. iii. Progress of Theoretical Physics, 35(6):10611073, 1966. doi: 10.1143/PTP.35.1061. URL http://ptp.oxfordjournals.org/ content/35/6/1061. abstract.

[159] M. Derrick et al. Measurement of elastic $\rho^{0}$ photoproduction at HERA. Z.Phys., C69:39-54, 1995. doi: 10.1007/s002880050004. URL http://arxiv.org/abs/ hep-ex/9507011. 
[160] M. Derrick et al. Measurement of elastic $\omega$ photoproduction at HERA. Z.Phys., C73:73-84, 1996. doi: 10.1007/s002880050297. URL http://dx.doi.org/10. $1007 / \mathrm{s} 002880050297$.

[161] M. Derrick et al. Measurement of elastic $\phi$ photoproduction at HERA. Phys.Lett., B377:259-272, 1996. doi: 10.1016/0370-2693(96)00172-4. URL http://www . sciencedirect.com/science/article/pii/0370269396001724.

[162] S. Chekanov et al. Exclusive photoproduction of $J / \Psi$ mesons at HERA. Eur.Phys.J., C24:345-360, 2002. doi: 10.1007/s10052-002-0953-7. URL http: //dx.doi.org/10.1007/s10052-002-0953-7.

[163] Claudia Ratti, Michael A. Thaler, and Wolfram Weise. Phases of qcd: Lattice thermodynamics and a field theoretical model. Phys. Rev. D, 73:014019, Jan 2006. doi: 10.1103/PhysRevD.73.014019. URL http://link.aps.org/doi/10. 1103/PhysRevD. 73.014019.

[164] M C Birse. Chiral symmetry in nuclei: partial restoration and its consequences. Journal of Physics G: Nuclear and Particle Physics, 20(10):1537, 1994. URL http://stacks . iop.org/0954-3899/20/i=10/a=003.

[165] G.I. Lykasov, W. Cassing, A. Sibirtsev, and M.V. Rzjanin. $\omega N$ final state interactions and $\omega$-meson production from heavy-ion collisions. The European Physical Journal A - Hadrons and Nuclei, 6:71-81, 1999. ISSN 1434-6001. URL http://dx.doi.org/10.1007/s100500050319. 10.1007/s100500050319.

[166] M. Gell-Mann, D. Sharp, and W. G. Wagner. Decay rates of neutral mesons. Phys. Rev. Lett., 8:261-262, Mar 1962. doi: 10.1103/PhysRevLett.8.261. URL http://link.aps.org/doi/10.1103/PhysRevLett.8.261.

[167] R. Machleidt, K. Holinde, and Ch. Elster. The bonn meson-exchange model for the nucleonnucleon interaction. Physics Reports, 149(1):1-89, 1987. ISSN 03701573. doi: http://dx.doi.org/10.1016/S0370-1573(87)80002-9. URL http://www . sciencedirect.com/science/article/pii/S0370157387800029.

[168] S. Fajfer and Robert J. Oakes. $V^{0} \rightarrow P^{0} P^{0} \gamma$ decay rates. Phys. Rev. D, 42: 2392-2395, Oct 1990. doi: 10.1103/PhysRevD.42.2392. URL http://link.aps . org/doi/10.1103/PhysRevD.42.2392.

[169] Nguyen D. Son and J. Sucher. Bound states of a relativistic two-body hamiltonian: Comparison with the bethe-salpeter equation. Phys. Rev., 161:1694-1694, Sep 1967. doi: 10.1103/PhysRev.161.1694. URL http://link.aps.org/doi/10 . 1103/PhysRev.161.1694. 
[170] Paul Singer. Decay mode $\omega \rightarrow 2 \pi+\gamma$. Phys. Rev., 128:2789-2792, Dec 1962. doi: 10.1103/PhysRev.128.2789. URL http://link.aps.org/doi/10.1103/PhysRev . 128.2789 .

[171] Paul Singer. Radiative $\rho$-meson decay. Phys. Rev., 130:2441-2448, Jun 1963. doi: 10.1103/PhysRev.130.2441. URL http://link.aps.org/doi/10.1103/PhysRev . 130.2441.

[172] Review of particle physics. The European Physical Journal C - Particles and Fields, 3(1-4):1-783, 1998. ISSN 1434-6044. doi: 10.1007/s10052-998-0104-x. URL http://dx.doi.org/10.1007/s10052-998-0104-x.

[173] K. Nakayama, A. Szczurek, C. Hanhart, J. Haidenbauer, and J. Speth. Production of $\omega$ mesons in proton-proton collisions. Phys. Rev. C, 57:1580-1587, Apr 1998. doi: 10.1103/PhysRevC.57.1580. URL http://link.aps.org/doi/ 10.1103/PhysRevC.57.1580.

[174] H. J. Lipkin. Quark models and high-energy scattering. Phys. Rev. Lett., 16: 1015-1019, May 1966. doi: 10.1103/PhysRevLett.16.1015. URL http://link. aps.org/doi/10.1103/PhysRevLett.16.1015.

[175] K. Kajantie and J.S. Trefil. Suppression of the photoproduction in the quark model. Physics Letters B, 24(2):106-107, 1967. ISSN 0370-2693. doi: http://dx. doi.org/10.1016/0370-2693(67)90360-7. URL http://www.sciencedirect.com/ science/article/pii/0370269367903607.

[176] A. Sibirtsev, W. Cassing, G.I. Lykasov, and M.V. Rzjanin. Reanalysis of antiproton production in proton-nucleus and nucleus-nucleus reactions at subthreshold energies. Nuclear Physics A, 632(1):131-152, 1998. ISSN 0375-9474. doi: 10.1016/S0375-9474(97)00809-9. URL http://www.sciencedirect.com/ science/article/pii/S0375947497008099.

[177] J. J. de Swart, M. C. M. Rentmeester, and R. G. E. Timmermans. $\pi N$ Newsletter, 13:96, 1997.

[178] A. Sibirtsev, K. Tsushima, and A.W. Thomas. A clue to the mechanism of $\Lambda K^{+}$ production in pp-reactions. Physics Letters B, 421(1-4):59-63, 1998. ISSN 03702693. doi: 10.1016/S0370-2693(98)00025-2. URL http://www.sciencedirect. com/science/article/pii/S0370269398000252.

[179] A. Sibirtsev, K. Tsushima, and A. W. Thomas. Charmonium absorption by nucleons. Phys. Rev. C, 63:044906, Mar 2001. doi: 10.1103/PhysRevC.63.044906. URL http://link. aps.org/doi/10.1103/PhysRevC.63.044906. 
[180] M.C. Abreu et al. Evidence for deconfinement of quarks and gluons from the $J / \Psi$ suppression pattern measured in $\mathrm{Pb}-\mathrm{Pb}$ collisions at the CERN-SPS. Physics Letters B, 477(1-3):28-36, 2000. ISSN 0370-2693. doi: http://dx.doi.org/10. 1016/S0370-2693(00)00237-9. URL http://www.sciencedirect.com/science/ article/pii/S0370269300002379.

[181] A. Sibirtsev, K. Tsushima, and A.W. Thomas. On studying charm in nuclei through antiproton annihilation. The European Physical Journal A - Hadrons and Nuclei, 6(3):351-359, 1999. ISSN 1434-6001. doi: 10.1007/s100500050353. URL http://dx.doi.org/10.1007/s100500050353.

[182] A. Sibirtsev, W. Cassing, and U. Mosel. Heavy meson production in proton-nucleus reactions with empirical spectral functions. Zeitschrift für Physik A Hadrons and Nuclei, 358:357-367, 1997. ISSN 0939-7922. URL http://dx.doi.org/10.1007/ s002180050339. 10.1007/s002180050339.

[183] I. González, F. Guzmán, and A. Deppman. Nuclear photoproduction of vector mesons within a monte carlo approach. Phys. Rev. C, 89:054613, May 2014. doi: 10.1103/PhysRevC.89.054613. URL http://link.aps.org/doi/10.1103/ PhysRevC. 89.054613.

[184] D. O. Caldwell, V. B. Elings, W. P. Hesse, G. E. Jahn, R. J. Morrison, F. V. Murphy, and D. E. Yount. Total photoabsorption cross sections up to $18 \mathrm{gev}$ and the nature of photon interactions. Phys. Rev. Lett., 23:1256-1260, Nov 1969. doi: 10.1103/PhysRevLett.23.1256. URL http://link.aps.org/doi/10.1103/ PhysRevLett.23.1256.

[185] R. Hashimoto, H. Fujimura, H. Fukasawa, Q.H. He, Y. Honda, et al. Study for the $\omega-N$ Interaction Through $\omega$ Photoproduction Near the Threshold. Few Body Syst., 54:1135-1140, 2013. doi: 10.1007/s00601-013-0658-5. URL http: //dx.doi.org/10.1007/s00601-013-0658-5.

[186] Stanley J. Brodsky, Ivan Schmidt, and Guy F. de Téramond. Nuclear-bound quarkonium. Phys. Rev. Lett., 64:1011-1014, Feb 1990. doi: 10.1103/PhysRevLett. 64.1011. URL http://link.aps.org/doi/10.1103/PhysRevLett.64.1011.

[187] G Krein. J/I in nuclear matter. Journal of Physics: Conference Series, 422(1): 012012, 2013. URL http://stacks.iop.org/1742-6596/422/i=1/a=012012.

[188] K. Tsushima, D. H. Lu, G. Krein, and A. W. Thomas. J/I-nuclear bound states. Phys. Rev. C, 83:065208, Jun 2011. doi: 10.1103/PhysRevC.83.065208. URL http://link.aps.org/doi/10.1103/PhysRevC.83.065208. 
[189] Kazuo Tsushima, D. H. Lu, Gastao Krein, and Anthony W. Thomas. J/I mass shift and $J /$ Inuclear bound state. AIP Conference Proceedings, 1354(1):39-44, 2011. doi: http://dx.doi.org/10.1063/1.3587583. URL http://scitation.aip. org/content/aip/proceeding/aipcp/10.1063/1.3587583.

[190] M. Effenberger, E. L. Bratkovskaya, W. Cassing, and U. Mosel. $e^{+} e^{-}$pairs from $\pi^{-}$A reactions reexamined. Phys. Rev. C, 60:027601, Jul 1999. doi: 10.1103/ PhysRevC.60.027601. URL http://link.aps.org/doi/10.1103/PhysRevC.60. 027601.

[191] R. Nasseripour et al. Search for medium modifications of the $\rho$ meson. Phys. Rev. Lett., 99:262302, Dec 2007. doi: 10.1103/PhysRevLett.99.262302. URL http: //link.aps.org/doi/10.1103/PhysRevLett.99.262302.

[192] M. Kotulla, , et al. Modification of the $\omega$ meson lifetime in nuclear matter. Phys. Rev. Lett., 100:192302, May 2008. doi: 10.1103/PhysRevLett.100.192302. URL http://link.aps.org/doi/10.1103/PhysRevLett.100.192302.

[193] D. Cabrera, L. Roca, E. Oset, H. Toki, and M.J. Vicente Vacas. Mass dependence of inclusive nuclear $\phi$ photoproduction. Nuclear Physics A, 733(12):130-141, 2004. ISSN 0375-9474. doi: http://dx.doi.org/10.1016/j.nuclphysa. 2003.12.012. URL http://www.sciencedirect.com/science/article/pii/ S0375947403019754.

[194] P. Mühlich and U. Mosel. Attenuation of $\phi$ mesons in $\gamma$ a reactions. Nuclear Physics A, 765(1-2):188-196, 2006. doi: http://dx.doi.org/10.1016/j.nuclphysa. 2005.11.007. URL http://www.sciencedirect.com/science/article/pii/ S0375947405011966.

[195] P. Mühlich and U. Mosel. $\omega$ attenuation in nuclei. Nuclear Physics A, 773(12):156-172, 2006. doi: http://dx.doi.org/10.1016/j.nuclphysa.2006.05.001. URL http://www.sciencedirect.com/science/article/pii/S0375947406001916.

[196] T. Ishikawa et al. $\phi$ photo-production from li, c, al, and cu nuclei at $E_{-} \gamma=1.5-$ 2.4GeV. Physics Letters B, 608(3-4):215-222, 2005. ISSN 0370-2693. doi: http: //dx.doi.org/10.1016/j.physletb.2005.01.023. URL http://www.sciencedirect. com/science/article/pii/S0370269305000560.

[197] M. Wood et al. Absorption of the $\omega$ and $\phi$ mesons in nuclei. Phys. Rev. Lett., 105:112301, Sep 2010. doi: 10.1103/PhysRevLett.105.112301. URL http://link. aps.org/doi/10.1103/PhysRevLett.105.112301.

[198] P. Mühlich, T. Falter, and U. Mosel. Inclusive $\omega$ photoproduction off nuclei. The European Physical Journal A - Hadrons and Nuclei, 20:499-508, 2004. 
ISSN 1434-6001. URL http://dx.doi.org/10.1140/epja/i2003-10166-3. 10.1140/epja/i2003-10166-3.

[199] M. Kaskulov, E. Hernandez, and E. Oset. Inclusive $\omega$ photoproduction from nuclei and $\omega$ in the nuclear medium. The European Physical Journal A - Hadrons and Nuclei, 31:245-254, 2007. ISSN 1434-6001. URL http://dx.doi.org/10.1140/ epja/i2006-10169-6. 10.1140/epja/i2006-10169-6. 